\title{
Aerodynamic, Aeroacoustic, and Aeroelastic Investigations of Airfoil-Vortex Interaction using Large-Eddy Simulation
}

By

Marcel Ilie

A thesis submitted to The Faculty of Graduate Studies and Research in partial fulfilment of the requirements for the degree of

\author{
Doctor of Philosophy \\ in Aerospace Engineering
}

Ottawa-Carleton Institute for Mechanical and Aerospace Engineering

\author{
Department of Mechanical and Aerospace Engineering \\ Carleton University \\ Ottawa, Ontario, Canada
}

Copyright $(\mathbb{C}$ Marcel Ilie

January 2008 


$\begin{array}{ll}\begin{array}{l}\text { Library and } \\ \text { Archives Canada }\end{array} & \begin{array}{l}\text { Bibliothèque et } \\ \text { Archives Canada }\end{array} \\ \begin{array}{l}\text { Published Heritage } \\ \text { Branch }\end{array} & \begin{array}{l}\text { Direction du } \\ \text { Patrimoine de l'édition }\end{array} \\ \begin{array}{l}\text { 395 Wellington Street } \\ \text { Ottawa ON K1A 0N4 } \\ \text { Canada }\end{array} & \begin{array}{l}\text { 395, rue Wellington } \\ \text { Ottawa ON K1A 0N4 } \\ \text { Canada }\end{array}\end{array}$

Your file Votre référence ISBN: 978-0-494-40524-6

Our file Notre référence

ISBN: 978-0-494-40524-6

NOTICE:

The author has granted a nonexclusive license allowing Library and Archives Canada to reproduce, publish, archive, preserve, conserve, communicate to the public by telecommunication or on the Internet, loan, distribute and sell theses worldwide, for commercial or noncommercial purposes, in microform, paper, electronic and/or any other formats.

The author retains copyright ownership and moral rights in this thesis. Neither the thesis nor substantial extracts from it may be printed or otherwise reproduced without the author's permission.
AVIS:

L'auteur a accordé une licence non exclusive permettant à la Bibliothèque et Archives Canada de reproduire, publier, archiver, sauvegarder, conserver, transmettre au public par télécommunication ou par l'Internet, prêter, distribuer et vendre des thèses partout dans le monde, à des fins commerciales ou autres, sur support microforme, papier, électronique et/ou autres formats.

L'auteur conserve la propriété du droit d'auteur et des droits moraux qui protège cette thèse. $\mathrm{Ni}$ la thèse ni des extraits substantiels de celle-ci ne doivent être imprimés ou autrement reproduits sans son autorisation.
In compliance with the Canadian Privacy Act some supporting forms may have been removed from this thesis.

While these forms may be included in the document page count, their removal does not represent any loss of content from the thesis.
Conformément à la loi canadienne sur la protection de la vie privée, quelques formulaires secondaires ont été enlevés de cette thèse.

Bien que ces formulaires aient inclus dans la pagination, il n'y aura aucun contenu manquant.

\section{Canada}




\section{Dedicated to my wife}




\section{Abstract}

In helicopters, vortices (generated at the tip of the rotor blades) interact with the next advancing blades during certain flight and manoeuvring conditions, generating undesirable levels of acoustic noise and vibration. These Blade-Vortex Interactions (BVIs), which may cause the most disturbing acoustic noise, normally occur in descent or high-speed forward flight. Acoustic noise characterization (and potential reduction) is one the areas generating intensive research interest to the rotorcraft industry.

Since experimental investigations of BVI are extremely costly, some insights into the BVI or AVI (2-D Airfoil-Vortex Interaction) can be gained using Computational Fluid Dynamics (CFD) numerical simulations. Numerical simulation of BVI or AVI has been of interest to CFD for many years. There are still difficulties concerning an accurate numerical prediction of BVI. One of the main issues is the inherent dissipation of CFD turbulence models, which severely affects the preservation of the vortex characteristics. Moreover this is not an issue only for aerodynamic and aeroacoustic analysis but also for aeroelastic investigations as well, especially when the strong (two-way) aeroelastic coupling is of interest.

The present investigation concentrates mainly on AVI simulations. The simulations are performed for Mach number, $\mathrm{Ma}=0.3$, resulting in a Reynolds number, $\operatorname{Re}=1.3 \times 10^{6}$, which is based on the chord, $\mathrm{c}$, of the airfoil (NACA0012).

Extensive literature search has indicated that the present work represents the first comprehensive investigation of AVI using the LES numerical approach, in the rotorcraft research community. The major factor affecting the aerodynamic coefficients and 
aeroacoustic field as a result of airfoil-vortex interaction is observed to be the unsteady pressure generated at the location of the interaction. The present numerical results show that the aerodynamic coefficients (lift, moment, and drag) and aeroacoustic field are strongly dependent on the airfoil-vortex vertical miss-distance, airfoil angle of attack, vortex characteristics, and aeroelastic response of airfoil to airfoil-vortex interaction.

A decay of airfoil-vortex interactions with the increase of vertical miss-distance and angle of attack was observed. Also, a decay of airfoil-vortex interactions is observed for the case of a flexible structure when compared with the case of a rigid structure. The decay of vortex core size produces a decrease in the aerodynamic coefficients. 


\section{Acknowledgements}

First and foremost, I would like to express my deepest gratitude to both of my advisors, Professor Fred Nitzsche and Professor Edgar Matida, for proposing this project and providing me guidance throughout the course of this thesis. They have given me considerable freedom and shown patience as I pursued several new ideas during the development of this research. I am really indebted to them for always being available for advice and corrections during the many stages of this Ph.D. research.

I would also like to thank very much to Neil McFadyen, our system administrator for his tedious and constant work, solving all the issue raised by the maintenance and improper functionality of the cluster.

I would like to acknowledge the financial support of High Performance Computing Virtual Laboratory through the Sun Microsystems of Canada Scholarship in Computational Sciences and Engineering, through the period of 2005-2006.

Most of all, I would like to express my love and great gratitude to my wife for her love, encouragement, and support. Her love and support has been my foundation throughout the course of this research, and have instilled in me the important balance between work and family. 


\section{Table of Contents}

\begin{tabular}{|c|c|}
\hline bstract & \\
\hline Acknowledgen & ments.... \\
\hline Table of Conte & ...iv \\
\hline List of Tables & $\ldots \mathrm{vi}$ \\
\hline List of Figures & \\
\hline Nomenclature & $\ldots x \mathrm{i}$ \\
\hline Chapter 1: & Introduction... \\
\hline 1.1 & Description of the problem .................. \\
\hline 1.2 & Motivation of the problem ................... \\
\hline 1.3 & Research Objectives................ \\
\hline 1.4 & 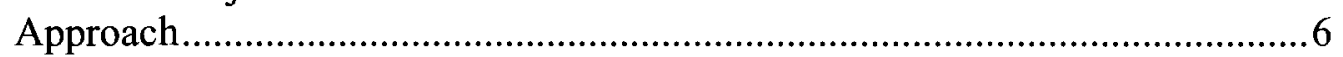 \\
\hline Chapter 2: & Background and Literature Review........... \\
\hline 2.1 & 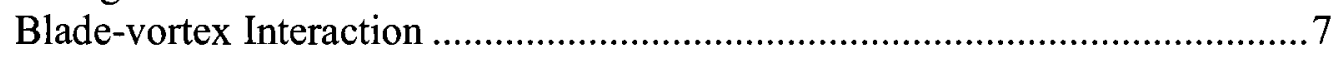 \\
\hline 2.2 & 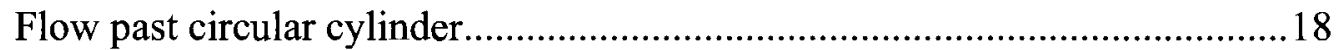 \\
\hline 2.3 & 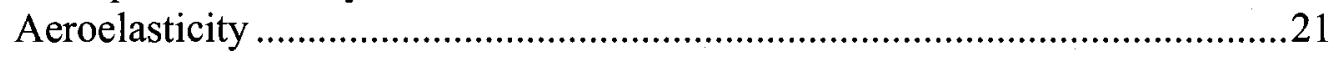 \\
\hline Chapter 3: & Numerical Methods for AVI and Turbulent Flows.. \\
\hline 3.1 & 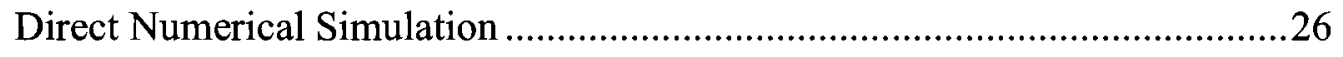 \\
\hline 3.2 & Reynolds Averaged Navier-Stokes Simulation (RANS) .. \\
\hline 3.2 .1 & The Shear Stress Transport Model (SST) ......................... \\
\hline 3.3 & 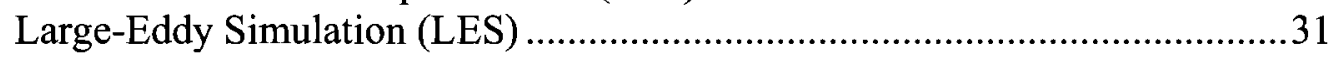 \\
\hline 3.3 .1 & Governing Equations ..................... \\
\hline 3.3 .2 & 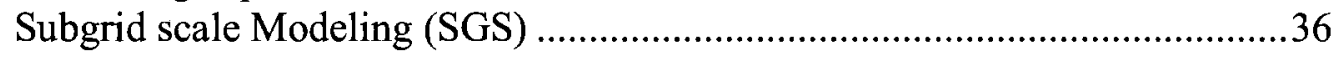 \\
\hline 3.3 .3 & 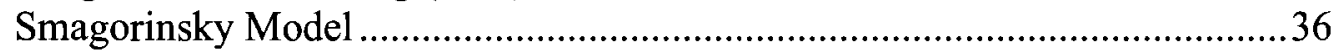 \\
\hline 3.3 .4 & Modified Smagorinsky Model ............................................ \\
\hline 3.3 .5 & Dynamic Subgrid scale Model...... \\
\hline 3.3 .6 & Aeroacoustic modeling using large-eddy simulation...... \\
\hline Chapter 4: & Numerical Simulation Results-Circular Cylinder........ \\
\hline 4.1 & 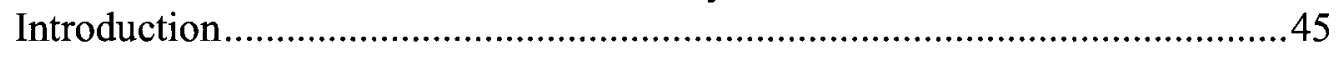 \\
\hline 4.2 & 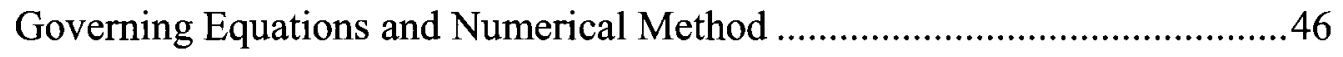 \\
\hline 4.3 & 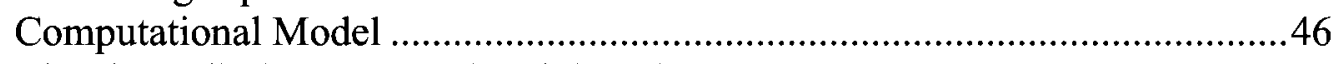 \\
\hline 4.3.1 & 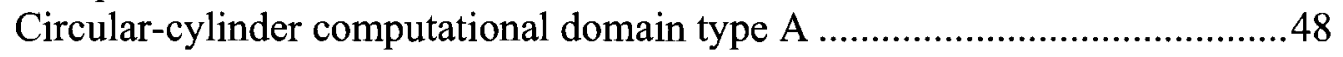 \\
\hline 4.3 .2 & 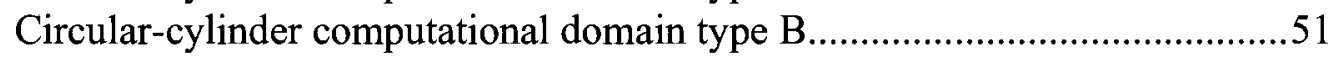 \\
\hline 4.3 .3 & 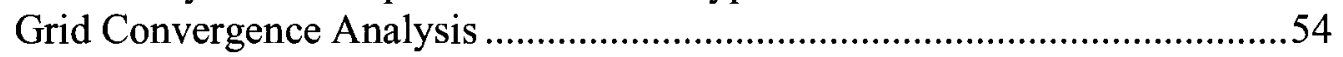 \\
\hline 4.4 & 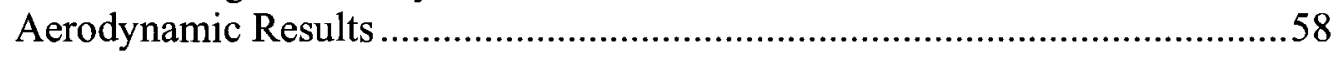 \\
\hline 4.4.1 & 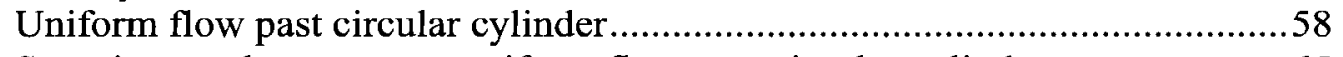 \\
\hline 4.4 .2 & Superimposed vortex on a uniform flow past circular cylinder.................. \\
\hline Chapter 5: & The Aerodynamics and Aeroacoustics of AVI...................... \\
\hline 5.1 & Introduction \\
\hline 5.2 & Governing Equations ... \\
\hline 5.3 & Computational Model .. \\
\hline & NACA0012 computational domain type A.......................... \\
\hline 5.3 .2 & 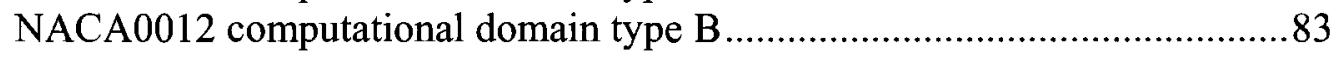 \\
\hline
\end{tabular}




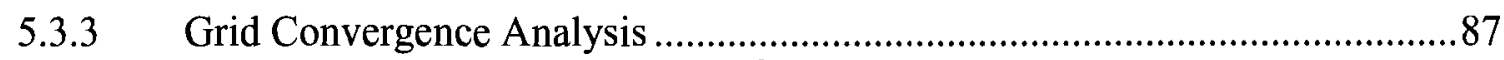

5.4 Aerodynamic Results (Airfoil at $0^{\circ}$ Angle of Attack) ....................................89

5.5 Aerodynamic Results (Airfoil at $5^{\circ}$ Angle of Attack) ....................................100

5.6 Aerodynamic Results (Airfoil at $10^{\circ}$ Angle of Attack) ..................................108

5.7 Aerodynamic Comparative Results $\left(0^{\circ}, 5^{\circ}\right.$, and $10^{\circ}$ Angle of Attack) ........113

$5.8 \quad$ Aerodynamic Results (Vortex Core Size)....................................................116

$5.9 \quad$ Aeroacoustic Results.................................................................................. 121

Chapter 6: Aerodynamic and Aeroelastic Investigations of Idealized Airfoil-Vortex Interaction........................................................ 156

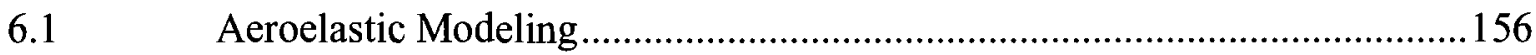

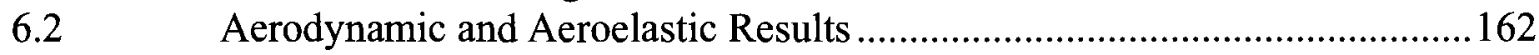

Chapter 7: Conclusions...................................................... 176

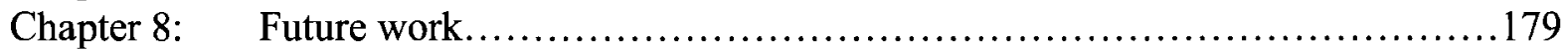




\section{List of Tables}

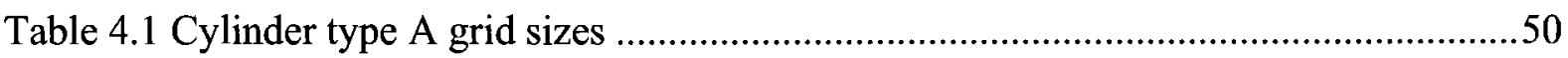

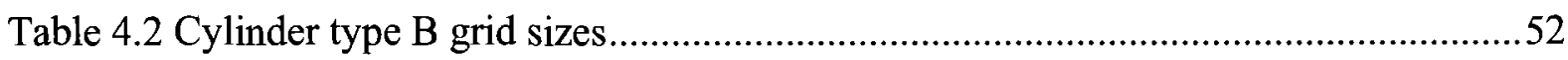

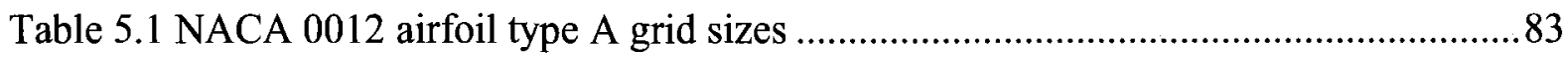

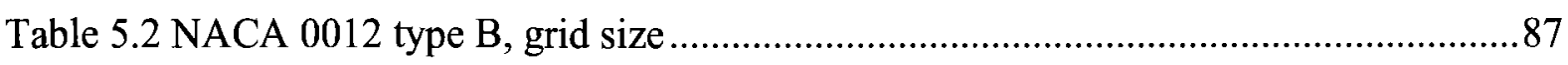




\section{List of Figures}

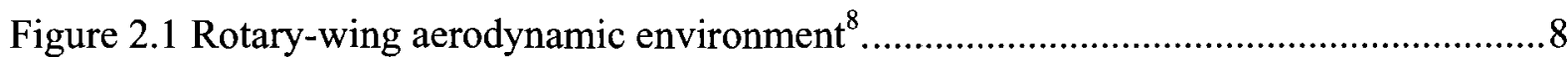

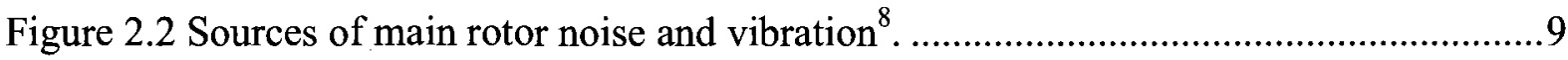

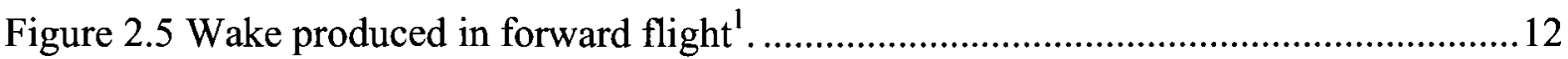

Figure 4.1 Cylindrical geometry, computational domain type A. ...........................................48

Figure 4.2 Schematic view of cylinder computational domain type A.....................................49

Figure 4.3 Schematic view of cylinder computational domain type A....................................49

Figure 4.4 Schematic view of cylinder computational domain type B....................................51

Figure 4.5 Schematic view of cylinder computational domain type B...................................52

Figure 4.6 Schematic view of cylinder computational domain type B...................................53

Figure 4.7 Schematic view of interrogation lines used for grid convergence analysis.............54

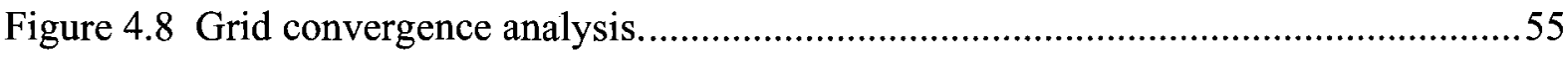

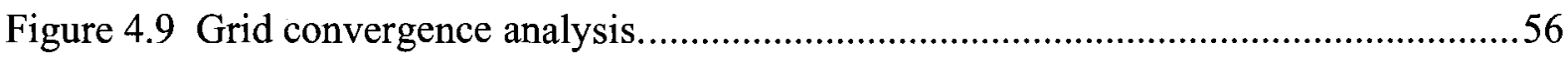

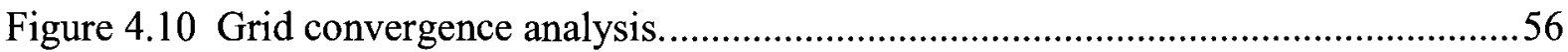

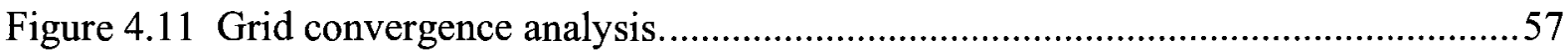

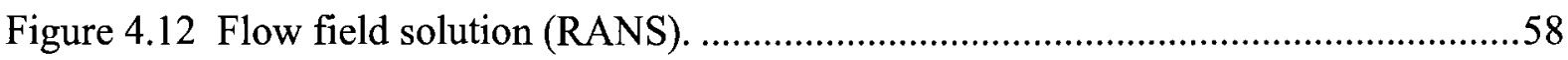

Figure 4.13 Pressure coefficient from RANS. .....................................................................59

Figure 4.14 Skin friction coefficient from RANS. ………………....................................59

Figure 4.15 Time developing fluid structures of a uniform flow past circular cylinder (LES).

Figure 4.16 Uniform flow past circular cylinder, time history of lift coefficient....................63

Figure 4.17 Uniform flow past circular cylinder, time history of drag coefficient. ................63

Figure 4.18 Time developing fluid flow structures of a superimposed vortex on uniform flow

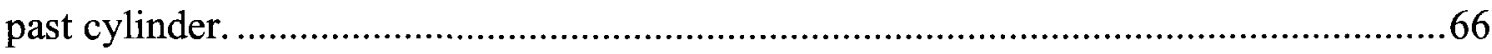

Figure 4.19 Superimposed vortex on uniform flow past a cylinder, time history of lift coefficient.

Figure 4.20 Superimposed vortex on uniform flow past a cylinder, time history of drag coefficient 
Figure 4.21 Time developing fluid structures of a superimposed vortex on a uniform flow past circular cylinder.

Figure 4.22 Time developing fluid structures of a superimposed vortex on a uniform flow past circular cylinder.

Figure 4.23 Superimposed clockwise rotating vortex on uniform flow past cylinder, lift coefficient comparison. .72

Figure 4.24 Superimposed clockwise rotating vortex on uniform flow past cylinder, drag coefficient comparison. .74

Figure 4.25 Superimposed clockwise rotating vortex on uniform flow past cylinder............75

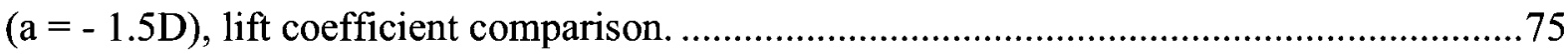

Figure 4.26 Superimposed clockwise rotating vortex on uniform flow past cylinder.............76

Figure 5.1 Schematic view of airfoil computational domain type A....................................... 81

Figure 5.2 Schematic view of airfoil computational domain type A.......................................82

Figure 5.3 Schematic view of airfoil computational domain type A.....................................8.

Figure 5.4 Schematic view of NACA0012 airfoil computational domain, type B...................84

Figure 5.5 Schematic close-view of NACA0012 airfoil computational domain, type B. .......84

Figure 5.6 Schematic view of NACA0012 airfoil, computational domain type B..................85

Figure 5.7 Schematic view of NACA0012 airfoil, size of the computational domain............86

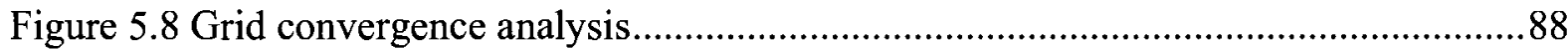

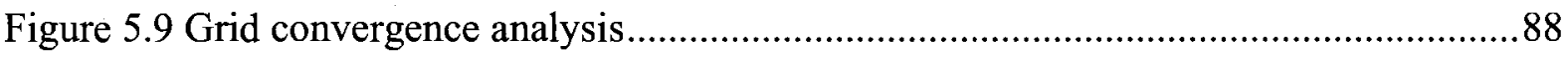

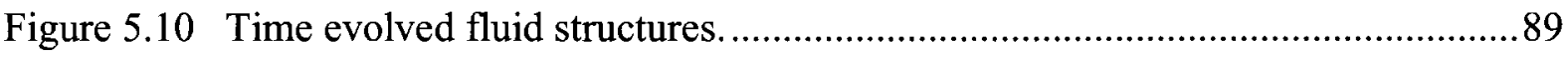

Figure 5.11 Time evolved fluid structures, pressure magnitude..............................................90

Figure 5.12 Time history of lift coefficient ………….....................................................92

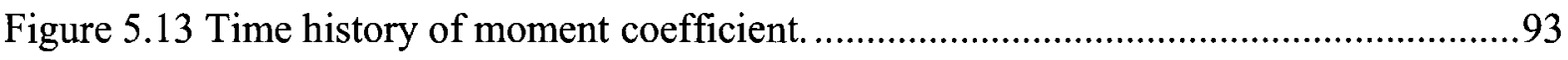

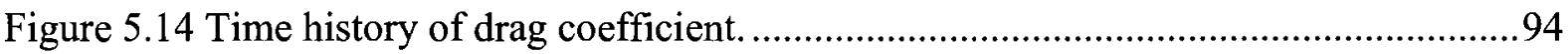

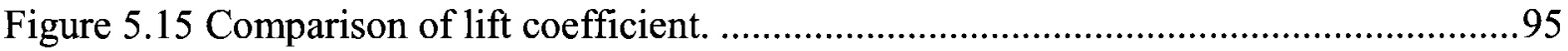

Figure 5.16 Comparison of moment coefficient. ...............................................................97

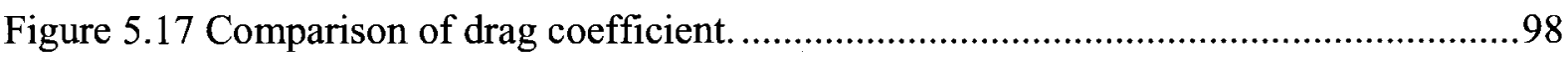

Figure 5.18 Time evolved fluid structures (velocity magnitude), angle of attack $\alpha=5^{\circ}$.....100

Figure 5.19 Time evolved fluid structures (pressure magnitude), angle of attack $\alpha=5^{\circ} \ldots . .101$

Figure 5.20 Comparison of lift coefficient, angle of attack $\alpha=5^{\circ}$...................................104 
Figure 5.21 Comparison of drag coefficient, angle of attack $\alpha=5^{\circ}$ 106

Figure 5.22 Time evolved fluid structures (velocity magnitude), angle of attack $\alpha=10^{\circ}$...108

Figure 5.23 Time evolved fluid structures (pressure magnitude), angle of attack $\alpha=10^{\circ} \ldots 109$

Figure 5.24 Comparison of lift coefficient, angle of attack $\alpha=10^{\circ}$. .111

Figure 5.25 Comparison of drag coefficient, angle of attack $\alpha=10^{\circ}$. 112

Figure 5.26 Comparison of lift coefficient, $\mathrm{h}=0.00 \mathrm{~m}$...................................................... 113

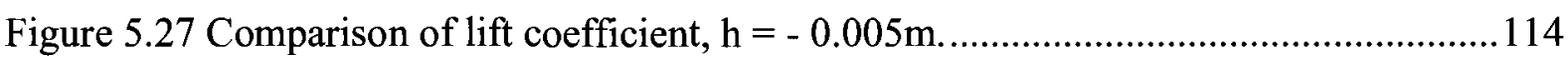

Figure 5.28 Comparison of drag coefficient, $h=0.00 \mathrm{~m}$.....................................................115

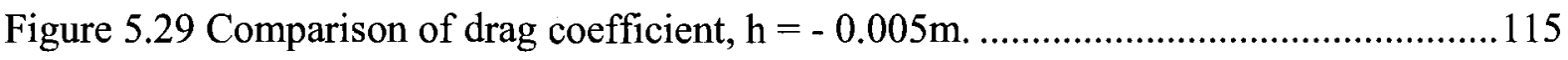

Figure 5.30 Time evolved fluid structures (velocity magnitude). ..........................................117

Figure 5.31 Time evolved fluid structures (pressure magnitude)..........................................118

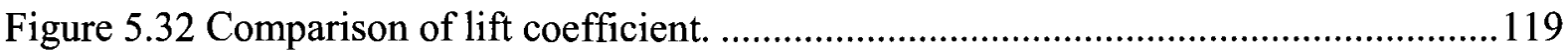

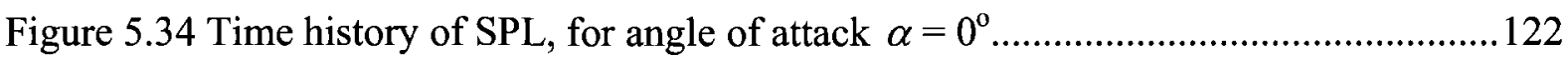

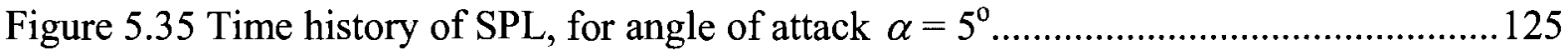

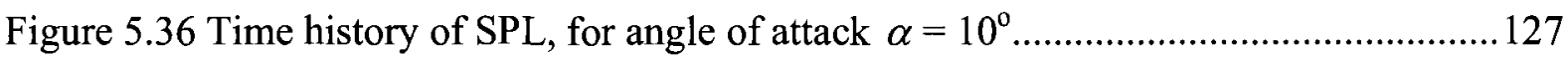

Figure 5.37 Schematic view of aeroacoustics interrogation paths........................................128

Figure 5.38 Comparison of SPL, $\alpha=0^{\circ}, \mathrm{h}=-0.00 \mathrm{~m}, \mathrm{x}=-0.05 \mathrm{~m} \ldots \ldots \ldots \ldots \ldots \ldots \ldots \ldots \ldots \ldots \ldots . .129$

Figure 5.39 Comparison of SPL, $\alpha=0^{\circ}, \mathrm{h}=-0.00 \mathrm{~m}, \mathrm{x}=0.1 \mathrm{~m} \ldots \ldots \ldots \ldots \ldots \ldots \ldots \ldots \ldots \ldots \ldots \ldots \ldots \ldots . .1131$

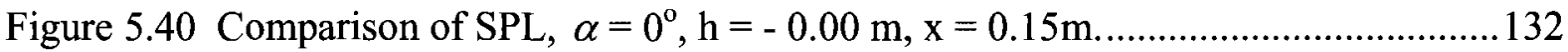

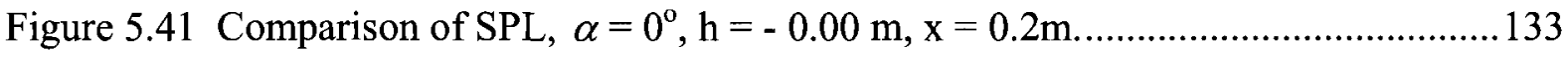

Figure 5.42 Comparison of SPL, $\alpha=0^{0}, \beta=10^{0} \mathrm{x}=-0.1 \mathrm{~m}$.............................................134

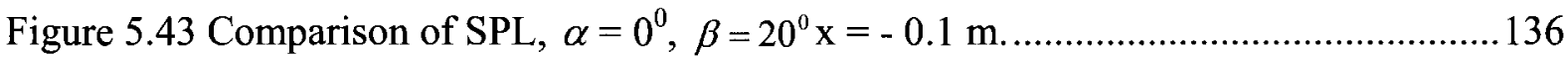

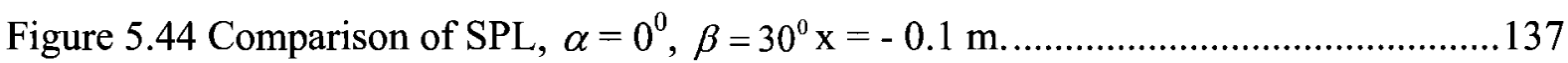

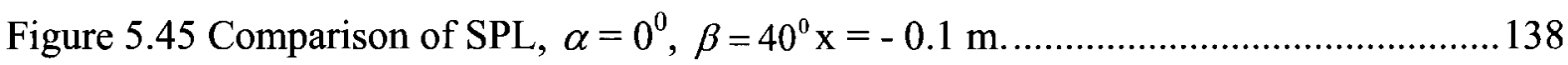

Figure 5.46 Comparison of SPL, $\alpha=5^{0}, \mathrm{~h}=-0.00 \mathrm{~m}, \mathrm{x}=-0.05 \mathrm{~m}$................................139

Figure 5.47 Comparison of SPL, $\alpha=5^{0}, \mathrm{~h}=-0.00 \mathrm{~m}, \mathrm{x}=-0.1 \mathrm{~m} . \ldots \ldots \ldots \ldots \ldots \ldots \ldots \ldots \ldots \ldots \ldots . .1140$

Figure 5.48 Comparison of SPL, $\alpha=5^{0}, \mathrm{~h}=-0.00 \mathrm{~m}, \mathrm{x}=-0.15 \mathrm{~m} . \ldots \ldots \ldots \ldots \ldots \ldots \ldots \ldots \ldots \ldots \ldots . . .114$

Figure 5.49 Comparison of SPL, $\alpha=5^{0}, \mathrm{~h}=-0.00 \mathrm{~m}, \mathrm{x}=-0.2 \mathrm{~m} \ldots \ldots \ldots \ldots \ldots \ldots \ldots \ldots \ldots \ldots \ldots \ldots \ldots \ldots \ldots \ldots \ldots \ldots \ldots . . . . .142$

Figure 5.50 Comparison of SPL for different angles of attack, $h=-0.00 \mathrm{~m}, \mathrm{x}=-0.05 \mathrm{~m} .143$

Figure 5.51 Time history of SPL, for different vortex core sizes..........................................144 
Figure 5.52 Comparison of SPL, $\mathrm{r}=0.5 \mathrm{c}, \alpha=0^{\circ}, \mathrm{h}=-0.00 \mathrm{~m}, \mathrm{x}=-0.05 \mathrm{~m} \ldots \ldots \ldots \ldots \ldots \ldots \ldots . .146$

Figure 5.53 Comparison of SPL, $\mathrm{r}=0.5 \mathrm{c}, \alpha=0^{\circ}, \mathrm{h}=-0.00 \mathrm{~m}, \mathrm{x}=-0.1 \mathrm{~m} \ldots \ldots \ldots \ldots \ldots \ldots \ldots . . .147$

Figure 5.54 Comparison of SPL, $\mathrm{r}=0.4 \mathrm{c}, \alpha=0^{\circ}, \mathrm{h}=-0.00 \mathrm{~m}, \mathrm{x}=-0.05 \mathrm{~m} \ldots \ldots \ldots \ldots \ldots \ldots \ldots . .148$

Figure 5.55 Comparison of SPL, $\mathrm{r}=0.4 \mathrm{c}, \alpha=0^{\circ}, \mathrm{h}=-0.00 \mathrm{~m}, \mathrm{x}=-0.1 \mathrm{~m} \ldots \ldots \ldots \ldots \ldots \ldots \ldots . . .149$

Figure 5.56 Comparison of SPL, $\mathrm{r}=0.3 \mathrm{c}, \alpha=0^{\circ}, \mathrm{h}=-0.00 \mathrm{~m}, \mathrm{x}=-0.05 \mathrm{~m} \ldots \ldots \ldots \ldots \ldots \ldots \ldots . .150$

Figure 5.57 Comparison of SPL, $\mathrm{r}=0.3 \mathrm{c}, \alpha=0^{\circ}, \mathrm{h}=-0.00 \mathrm{~m}, \mathrm{x}=-0.1 \mathrm{~m} \ldots \ldots \ldots \ldots \ldots \ldots \ldots . .151$

Figure 5.58 Comparison of SPL, $\alpha=0^{\circ}, \mathrm{h}=-0.00 \mathrm{~m}, \beta=15^{0}, \mathrm{x}=-0.05 \mathrm{~m} \ldots \ldots \ldots \ldots \ldots \ldots \ldots . .152$

Figure 5.59 Comparison of SPL, $\alpha=0^{\circ}, \mathrm{h}=-0.00 \mathrm{~m}, \beta=15^{0}, \mathrm{x}=-0.1 \mathrm{~m} \ldots \ldots \ldots \ldots \ldots \ldots \ldots \ldots . .153$

Figure 5.60 Comparison of SPL, $\alpha=0^{\circ}, \mathrm{h}=-0.00 \mathrm{~m}, \beta=30^{\circ}, \mathrm{x}=-0.05 \mathrm{~m} \ldots \ldots \ldots \ldots \ldots \ldots \ldots . . .154$

Figure 5.61 Comparison of SPL, $\alpha=0^{\circ}, \mathrm{h}=-0.00 \mathrm{~m}, \beta=30^{\circ}, \mathrm{x}=-0.1 \mathrm{~m} \ldots \ldots \ldots \ldots \ldots \ldots \ldots . . .155$

Figure 6.2 Blade-vortex miss-distance representation........................................................159

Figure 6.3 Schematic view of SDOF pitching idealized airfoil..........................................159

Figure 6.5 Time evolved fluid structures (rigid idealized airfoil), magnitude pressure. ....... 165

Figure 6.6 Time history of SPL, for an idealized airfoil...................................................... 167

Figure 6.7 Time history of lift coefficient (rigid structure) . ..................................................168

Figure 6.8 Comparison of lift coefficient (rigid structure) ..................................................169

Figure 6.9 Time history of lift coefficient (flexible structure)...............................................171

Figure 6.10 Lift coefficient comparison (flexible structure). ................................................172

Figure 6.11 Lift coefficient comparison, vertical miss-distance $\mathrm{h}=0.00 \mathrm{~m}$....................... 173

Figure 6.12 Lift coefficient comparison, vertical miss-distance $\mathrm{h}=-0.005 \mathrm{~m}$........................ 174

Figure 8.1 Multi beam-like structure model. .................................................................. 179

Figure 8.2 Airfoil multi beam-like structure model.........................................................180 


\section{Nomenclature}

\section{Quantity Description}

\begin{tabular}{|c|c|}
\hline $\mathrm{a}$ & cylinder-vortex horizontal miss-distance \\
\hline $\mathrm{B}$ & body force \\
\hline $\mathrm{C}$ & Courant Number \\
\hline $\mathrm{Cd}$ & drag coefficient \\
\hline $\mathrm{Cl}$ & lift coefficient \\
\hline $\mathrm{Cm}$ & moment coefficient \\
\hline $\mathrm{c}$ & airfoil chord \\
\hline$c_{\mu}$ & Parameter \\
\hline $\mathrm{D}$ & cylinder diameter \\
\hline$D^{\prime}$ & drag force per unit span \\
\hline$f^{d}$ & Diffusive flux \\
\hline $\mathrm{k}$ & turbulent kinetic energy \\
\hline$L$ & length \\
\hline $\mathrm{L}^{\prime}$ & lift force per unit span \\
\hline M & mass \\
\hline$P$ & static pressure \\
\hline $\bar{P}$ & Mean component of the fluid pressure \\
\hline$P^{\prime}$ & Turbulent component of the fluid pressure \\
\hline $\mathrm{q}$ & displacement \\
\hline $\mathrm{r}$ & vortex core radius \\
\hline $\mathrm{t}$ & time \\
\hline$u_{x}, u_{y}, u_{z}$ & Velocity components \\
\hline$C_{s}$ & Model parameter \\
\hline$L_{i}$ & Characteristic length scale \\
\hline$R_{i j}$ & Correlation tensor \\
\hline
\end{tabular}




$\begin{array}{cl}\mathrm{R} & \text { Reynolds stress tensor } \\ \mathrm{Re} & \text { Reynolds number } \\ S_{i j} & \text { Strain rate tensor } \\ \mathrm{S}_{\mathrm{t}} & \text { Strouhal number } \\ \dot{V} & \text { Volume flux } \\ \alpha & \text { angle of attack } \\ \beta & \text { spanning angle } \\ \delta_{i j} & \text { Kronecker symbol } \\ v & \text { kinematic viscosity } \\ \eta & \text { Kolmogorov length } \\ \rho & \text { Density } \\ v_{t} & \text { Turbulent viscosity } \\ \tau_{i j} & \text { Reynolds stress tensor } \\ \tau & \text { Kolmogorov time } \\ \varepsilon & \text { Rate of dissipation } \\ \tau_{i j}^{S} & \text { Stress tensor }\end{array}$

\section{Abbreviations}

$\begin{array}{ll}\text { AVI } & \text { Airfoil-Vortex Interaction } \\ \text { BVI } & \text { Blade-Vortex Interaction } \\ \text { CFD } & \text { computational fluid dynamics } \\ \text { CSD } & \text { computational structural dynamics } \\ \text { DNS } & \text { Direct Numerical Simulation } \\ \text { FSI } & \text { fluid-structure interaction } \\ \text { HIS } & \text { high impulsive sound } \\ \text { IBC } & \text { individual blade control } \\ \text { LES } & \text { Large-Eddy Simulations }\end{array}$




$\begin{array}{ll}\text { RANS } & \text { Reynolds Averaged Navier-Stokes } \\ \text { SDOF } & \text { single degree-of-freedom } \\ \text { SPL } & \text { sound pressure level } \\ \text { SST } & \text { shear stress transport } \\ \text { TAMI } & \text { tip air mass injection } \\ \text { URANS } & \text { Unsteady Reynolds Averaged Navier-Stokes }\end{array}$




\section{Chapter 1: Introduction}

This manuscript presents a comprehensive research regarding the aerodynamic, aeroacoustic, and aeroelastic investigations of a 2-D blade-vortex mechanism of interaction or airfoilvortex interaction (AVI). In this chapter the following topics will be discussed as follows: the description of the problem (Section 1.1), the motivation for the present research (Section 1.2) the objective of the research (Section 1.3), and the approach to be used for the present research (Section 1.4). Chapter 2 reviews the literature and background relevant to this endeavour. Chapter 3 is an introduction to numerical methods used in Computational Fluid Dynamics. Chapter 4 presents the numerical approach for the aeroelastic modeling. In chapter 5 the numerical aerodynamic results for the case of a circular cylinder are presented. Chapter 6 presents the numerical results of the aerodynamic and aeroacoustic investigations for the case of NACA0012 airfoil. In chapter 7 the numerical approach and results of an aeroelastic investigation based on an idealized airfoil (flat plate) are presented. The concluding remarks are presented in chapter 8 .

\subsection{Description of the problem}

In rotorcraft, blade-vortex interaction (BVI) is one of the main sources of noise and vibrations, and comprises one of the most complex unsteady flow features of helicopter rotor. Besides forward flight, BVI noise also occurs during landing-descending flight, and sometimes in manoeuvring flight, when the rotating blades pass in close proximity to the previously shed rotor tip vortices. When a vortex is shed from the rotating blade tip (due to 
pressure differences in between the lower and upper part of the blade) and convected downstream, it is intersected by the next advancing rotor blade as shown in Figure 1.1.

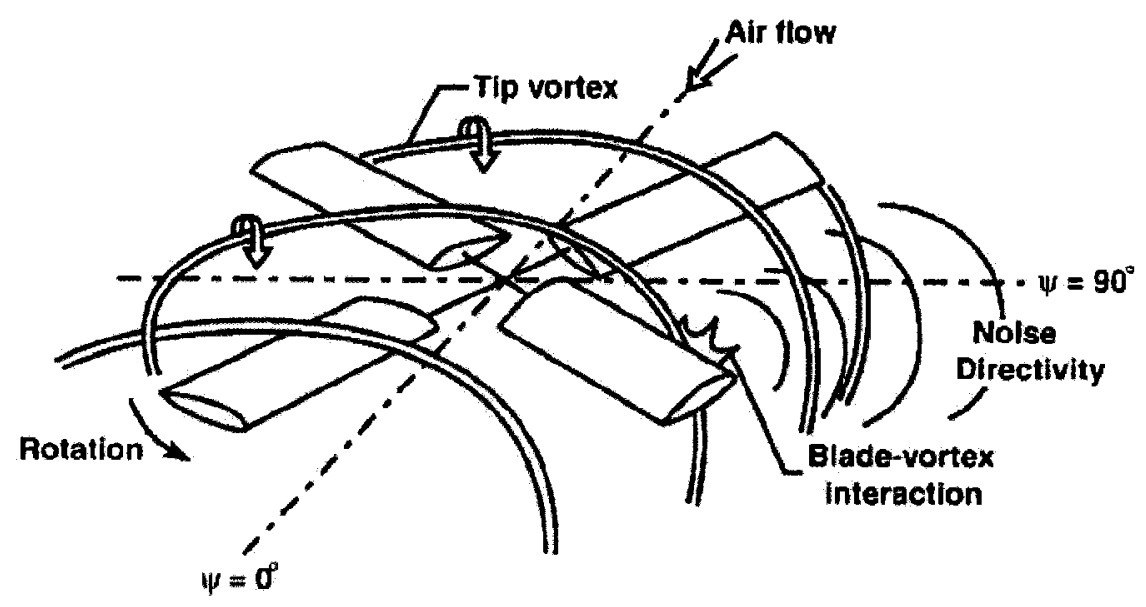

Figure 1.1 Schematic showing the BVI phenomenon and the radiated noise directivity ${ }^{8}$.

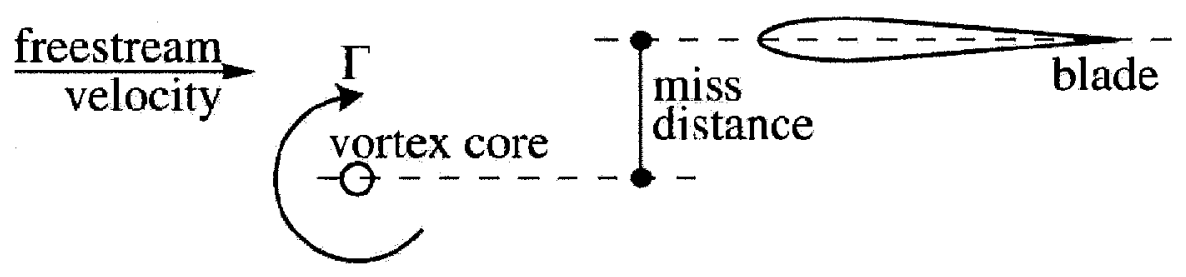

Figure 1.2 Blade-vortex miss-distance representation.

There are several types of BVI such as orthogonal BVI (vortex axis is perpendicular to the blade axis), oblique BVI (vortex axis is oblique respect to the blade axis), tail rotor BVI. However out of all types of BVI the parallel BVI was identified as the strongest. Strong interactions which result in strong chordwise temporal pressure variations are caused by a vortex whose axis is parallel (or nearly parallel) to the spanwise axis of the blade. Figure 1.2 shows the schematic of the start of a BVI, when the vortex is at an upstream location and is 
moving toward the leading edge of the blade. Notice the vertical miss-distance between the vortex core center and the chord line of the blade.

Numerical simulation of BVI has been of interest to CFD for many years. There are still difficulties concerning an accurate numerical prediction of BVI. One of the main issues is the inherent numerical dissipation of CFD models, which severely affects the preservation of the vortex characteristics. Accurate prediction of BVI aerodynamic loads and aeroacoustics using URANS (Unsteady Reynolds Averaged Navier-Stokes) is known to be very challenging due to the complex unsteady flow dynamics, involving boundary layer development on the suction side and flow separation. The use of Reynolds Averaged NavierStokes (RANS) methods, significantly rely on turbulence models to represent all the relevant turbulence scales. RANS methods predict the acoustic noise using the mean flow properties. Due to the fact that noise generation is a multi-scale problem, involving a wide range of length and time scales, the use of RANS-based prediction methods remains limited. Although RANS methods are useful for predicting the aerodynamic coefficients, holding accurately up to some extent, they are usually not suitable or reliable for accurate noise prediction.

The recent improvements in the processing speed of computers make the applicability of Direct Numerical Simulation (DNS) and Large-eddy simulation (LES) to turbulent flows more feasible. However, due to a wide range of length and time scales present in turbulent flows, the use of DNS is still limited to low-Reynolds number flows and relatively simple geometries. It is known that the number of grid points required for DNS is proportional to $\mathrm{Re}^{9 / 4}$. Direct Numerical Simulation of high-Reynolds number flows of practical interest would require high grid resolution that is far beyond the capability of the most powerful computers available nowadays. In order to overcome the grid requirement issues, turbulence 
has to be modeled in order to perform simulations for problems of practical interest. Largeeddy simulation, with a lower computational cost, is a promising alternative method to DNS, for simulations of high Reynolds number flows. LES methods are capable of simulating flows at high Reynolds number. In large-eddy simulation, the large scales are directly solved, while the small scales are modeled. Since noise generation is an unsteady process, LES is probably the most affordable computational tool to be used, since it is the only way, other than DNS, to obtain a time-accurate unsteady solution. The present analysis is based on a large-eddy simulation approach.

\subsection{Motivation of the problem}

Over the past years, the use of helicopters has increased substantially in both military and commercial sectors providing motivation for extensive research to more accurately estimate the blade aerodynamic loads, to further improve helicopter performance and stability and to reduce helicopter noise. The need for helicopter noise reduction represents in fact one of the strongest current motivations for research programs targeting at an increased understanding of rotor flow physics. Over the past years, progress has been made in BVI (Blade Vortex Interaction) noise prediction. Recently, the growing public demand for more stringent noise regulations has led the rotorcraft industry to develop innovative procedures to reduce BVI noise in the vicinity of urban areas. ${ }^{6-25}$ In addition to the adverse public opinion concerning acoustic noise; the BVI-induced vibratory loads increase pilot workload, reduce component fatigue life, and increase maintenance costs. Thus, the issues of vibration and noise prediction, and its reduction to the lowest possible levels are of primary importance to the 
helicopter designer. The motivation of the present research is based on the stringent need for a better understanding of the rotor flow physics, this being one of the main factors responsible for helicopter noise and vibrations.

\subsection{Research Objectives}

The main objective of the present work is to investigate the airfoil-vortex mechanism of interaction, from an aerodynamic, aeroacoustic and aeroelastic point of view based on several parameters such as airfoil-vortex vertical miss-distance, airfoil angle of attack, vortex characteristics and aeroelastic response to AVI. Also the present thesis focuses on the investigation of a CFD based numerical aeroelastic model that will help to define the relationship between the structure deformation and the physical characteristics of the turbulent flow.

Researchers face challenges unique to their method of solving the fluid-structure interaction (FSI) problem through analytical, numerical or experimental means. Current numerical and analytical techniques model the fluid flow using simplified assumptions, typically based on time averaged equations, which do not provide instantaneous values. The CFD codes based on Reynolds-Navier Stokes (RANS) fluid models do not compute the instantaneous fluctuations in the turbulent flow. Experimental solutions are too expensive and time consuming. It can be also difficult to isolate the vibrations induced by pressure fluctuations alone. Because of these challenges, accurately quantifying the vibrations induced by the pressure fluctuations alone has not yet been accomplished. 
Although the methods described above are insufficient to solve computationally the FluidStructure (FSI) problem, there are numerical techniques that are sufficient to model the fluid flow alone. These techniques are based on what is known as large-eddy simulations (LES). Large-eddy simulation is modeling the fluid flow by specially filtering the governing flow equations and solving for a local-averaged velocity rather than a time-averaged velocity, which provides the pressure instantaneous values. Therefore coupling a LES based fluid model with a structural model will provide the capability necessary to analyze the turbulentinduced phenomena. One of the objectives of this research is to develop an aeroelastic model based on LES, which accounts for instantaneous pressure fluctuations in the flow field.

\subsection{Approach}

The aerodynamic and aeroacoustic numerical investigation of Airfoil-Vortex Interaction is based on a large-eddy simulation approach. The aeroelastic numerical investigation of Airfoil-Vortex Interaction is based on a strong (two-way) fluid-structure coupling approach using large-eddy simulation. In this sense a beam-like structure approach will be used for the modeling of the blade structure. The structure is assumed to consist of multiple beam-like structures. The structure is solved using the Finite Element Method approach. The foundation of the proposed research is based on numerical simulations for which two different numerical codes will be used in order to simulate the Airfoil-Vortex Interaction. One numerical code (CFD) simulates the fluid flow and the other Computational Structural Dynamics (CSD) simulates the solid structure. The aeroelastic algorithm is based on the mesh deformation approach. 


\section{Chapter 2: Background and Literature Review}

\subsection{Blade-vortex Interaction}

Flow around structures, ranging from clarinet reeds to skyscrapers, can cause destructive vibrations as well as useful motions. Flow-induced vibrations have become increasingly important in recent years because designers are using materials to their limits, causing structures to become progressively lighter, more flexible, and more prone to vibration. The fluid flow and the structure are coupled through the force exerted on the structure by the fluid. The fluid forces cause the structure to deform. As the structure deforms, its orientation to the flow changes and the fluid force may change as well. The structure force on the fluid can synchronize vortices in the wake, producing large-amplitude vibration.

In the analysis of flow-induced vibration, models are generated for both structure and fluid. Since most structures are near-linear in deformation with increasing load, the structures are modeled as linear oscillators. If only a single structural degree of freedom is excited, the structural motion is described by a single linear equation with fluid forcing. If more than one degree of structural freedom is present, linear systems of equations are required. Fluid models are more difficult. Fluid mechanics is an inherently nonlinear, multi-degree-of-freedom phenomenon. Acoustic noise or undesirable sound is often the result of operating propulsive aerodynamic systems of which aircraft is one example. Fixed-wing aircraft generates noise through the engine and airframe which typically increases with the thrust level and aircraft speed. In addition to these factors rotorcrafts are equipped with a complex rotor system which is the main source of thrust and control as well the associated noise. 
The problems raised by the blade-vortex interaction are:

- High vibration loads

- Noise generation

- Poor performance of helicopter

- Helicopter instability

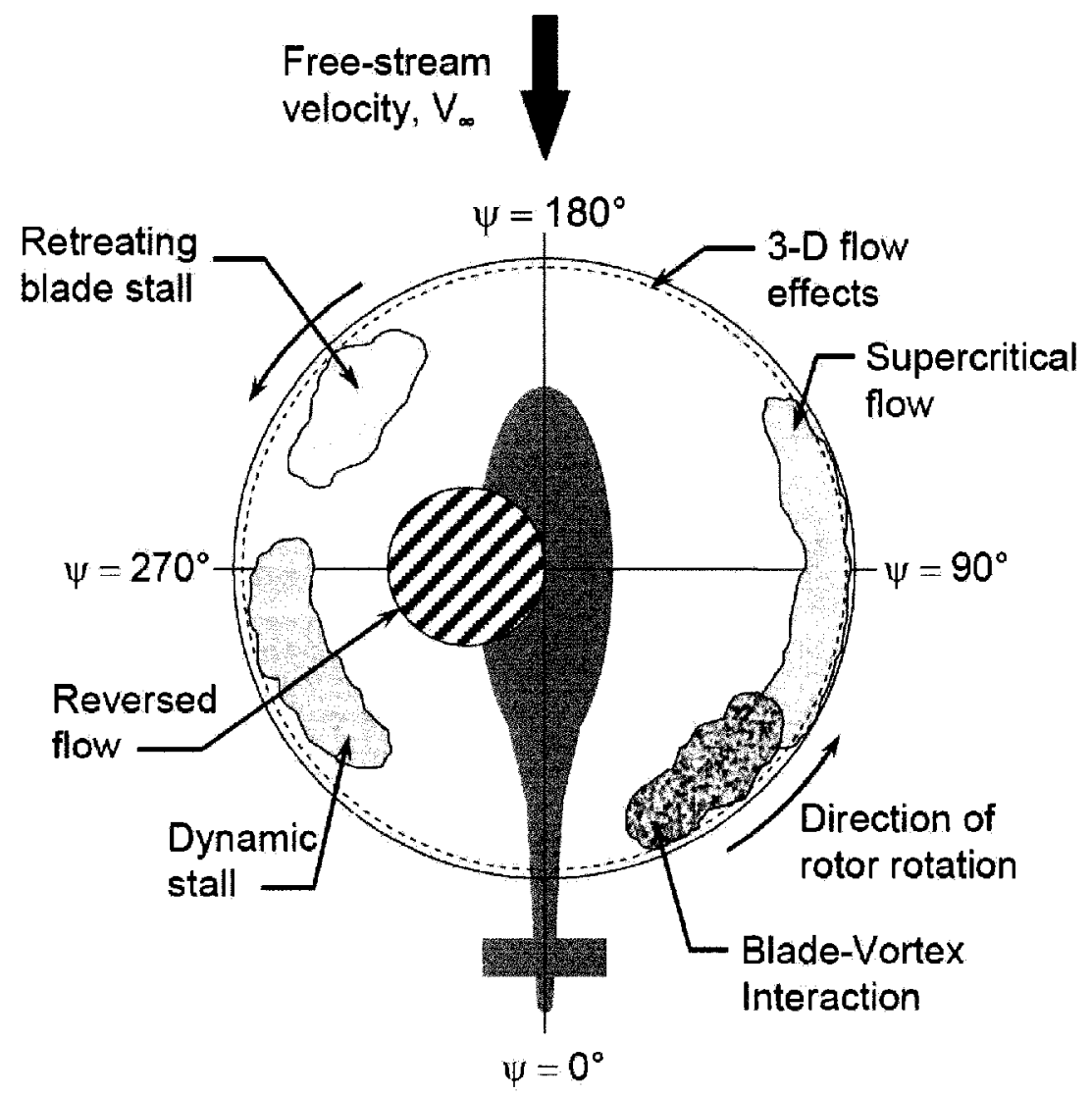

Figure 2.1 Rotary-wing aerodynamic environment ${ }^{1}$.

Figure 2.1 presents a schematic view of the helicopter rotor disc along with all the aerodynamics phenomena that occur during helicopter flight. Hence, the advancing side of 
the blades is associated with transonic flow (compressible flow) and blade-vortex interaction phenomena. The blade-vortex interaction occurs at azimuth angle of about $35^{\circ}$. The retreating side of the blades is associated with blade stall and dynamic stall. A region of reverse flow can be identified near the helicopter hub as seen in Figure 2.1. The reverse flow is causing an acceleration of the blade, due to the negative generated drag.

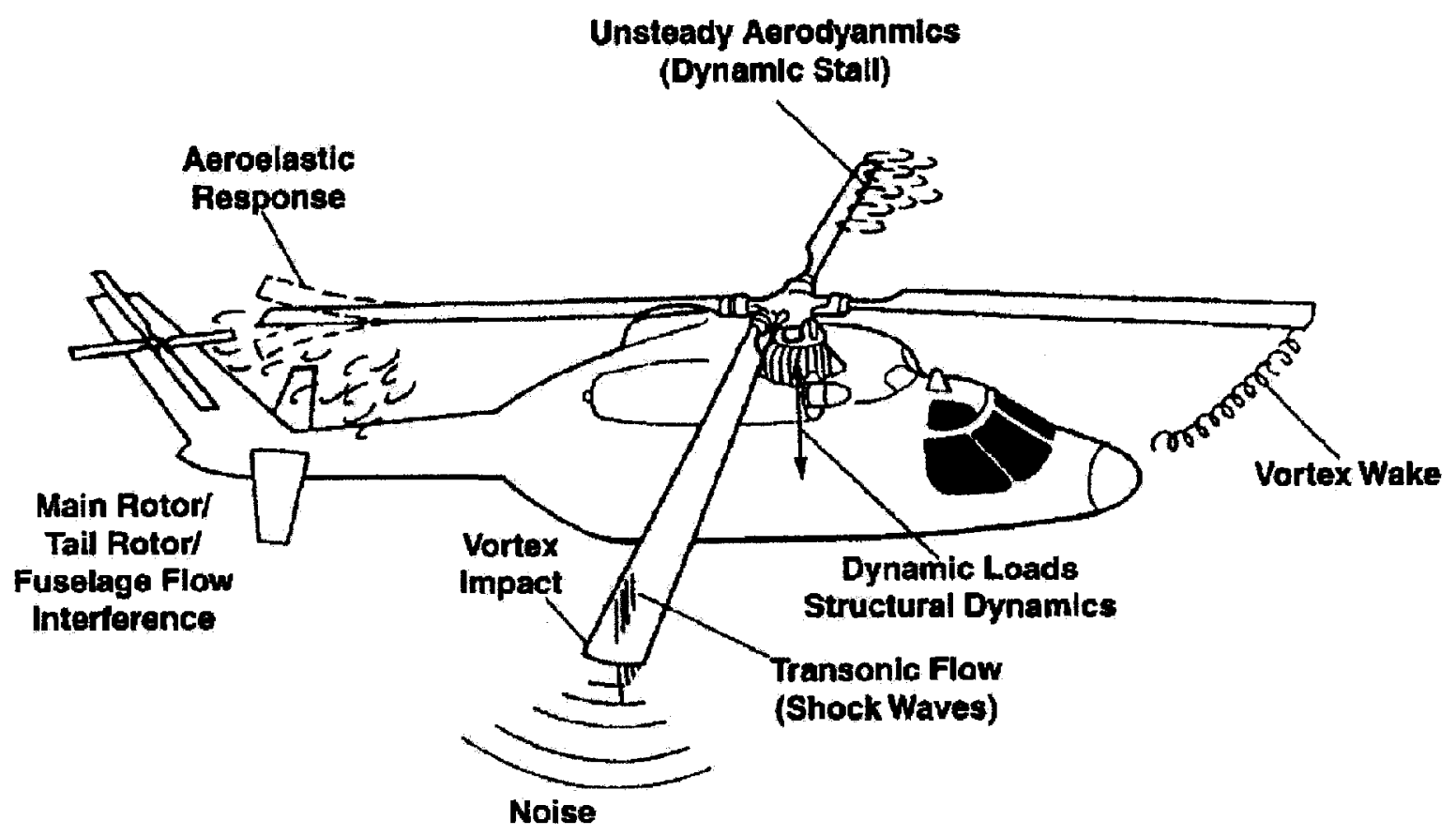

Figure 2.2 Sources of main rotor noise and vibration ${ }^{1}$. 


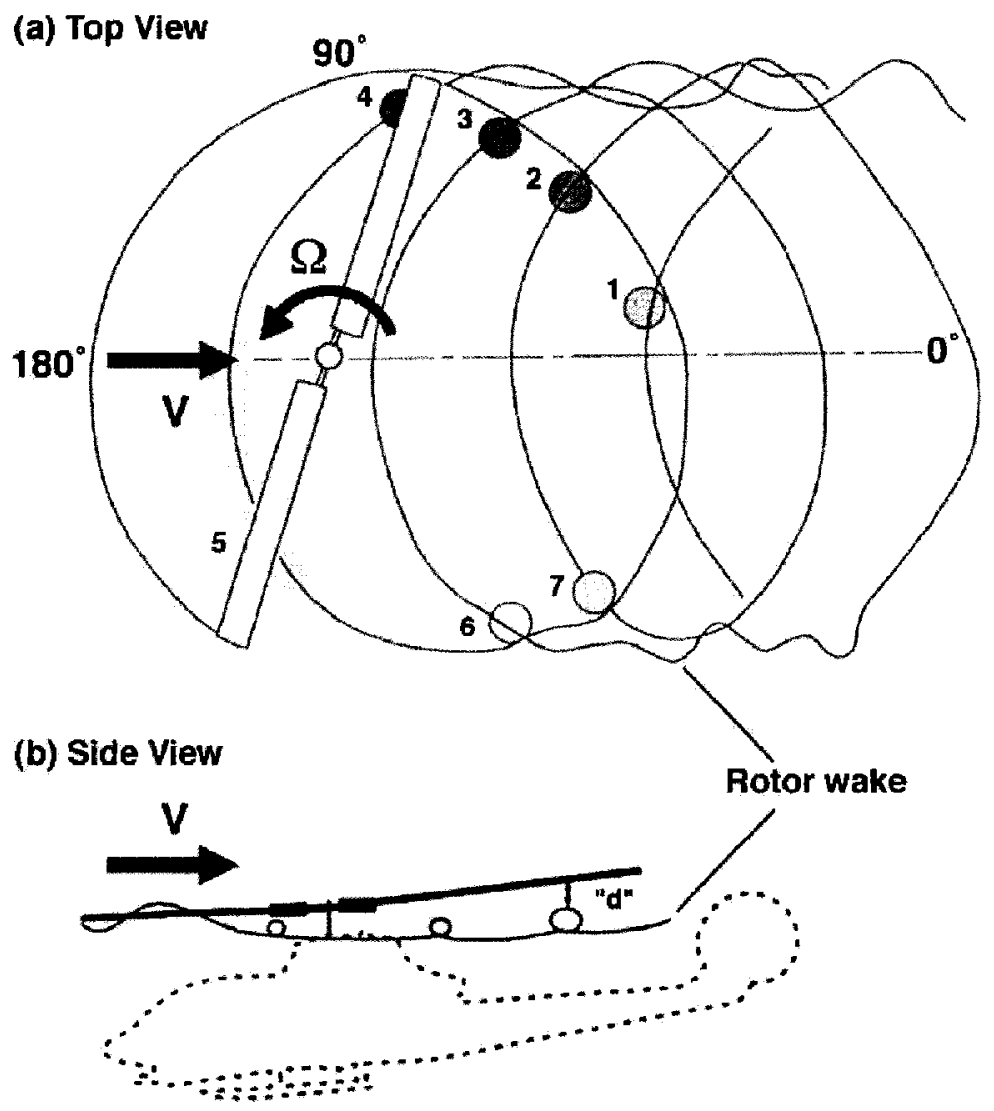

Figure 2.3 Helicopter BVI geometry for a two-bladed rotor: top and side view'

From Figures 2.2 and 2.3, it can be seen that during helicopter flight, vortices are formed at the tip of the rotor blade. These vortices induce sharp periodic aerodynamic disturbances on the next advancing blades, generating highly impulsive BVI noise. The radiated noise is dependent on the blade-vortex vertical miss-distance and the characteristics of previously shed vortex. BVI is most prominent during slow-speed descent, since during this phase of the flight, the vortex is more likely to interact with the rotor blades. BVI noise is usually radiated downward and dominates other noise sources when it occurs; during most landing descent approach conditions. When a vortex is shed from the rotating blade tip and convected downstream, it is intersected by the next advancing rotor blade. At the start of BVI, the 
vortex is at an upstream location and travels toward the airfoil leading edge, as schematically presented in Figure 2.2 and 2.3. As the vortex approaches the blade, it induces a downwash on the blade and causes the leading-edge stagnation point to move along the upper surface. This results into an increase in pressure over the blade upper surface, due to a decrease of velocity. At the lower surface of the airfoil, the flow is accelerated and a region of low pressure is observed. As the vortex passes the leading edge, it induces an upwash on the airfoil. As a result, the stagnation point moves from the upper surface to the leading edge and then toward the lower surface. The movement of the stagnation point influences the timevarying lift on the blade. The rate of change of lift is related to the pressure propagated to an observer. The unsteady vortex motion induces pressure fluctuations in the proximity of the blade which degenerate into a series of expansion/compression waves, which propagate upstream.

From Figure 2.2 it is evident that the flow-blade interaction is associated with the aeroelastic response of the blade. Recent studies have shown that the aeroelastic response of the blade has a significant impact on the blade-vortex mechanism of interaction. ${ }^{2}$ 


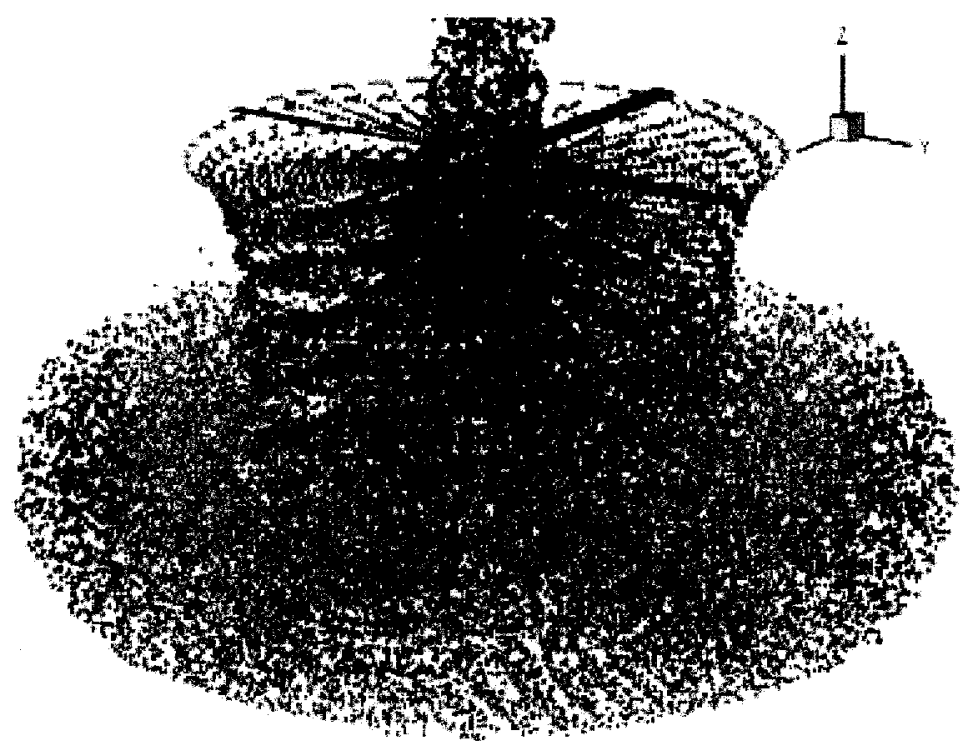

Figure 2.4 Wake produced in hover ${ }^{2}$.

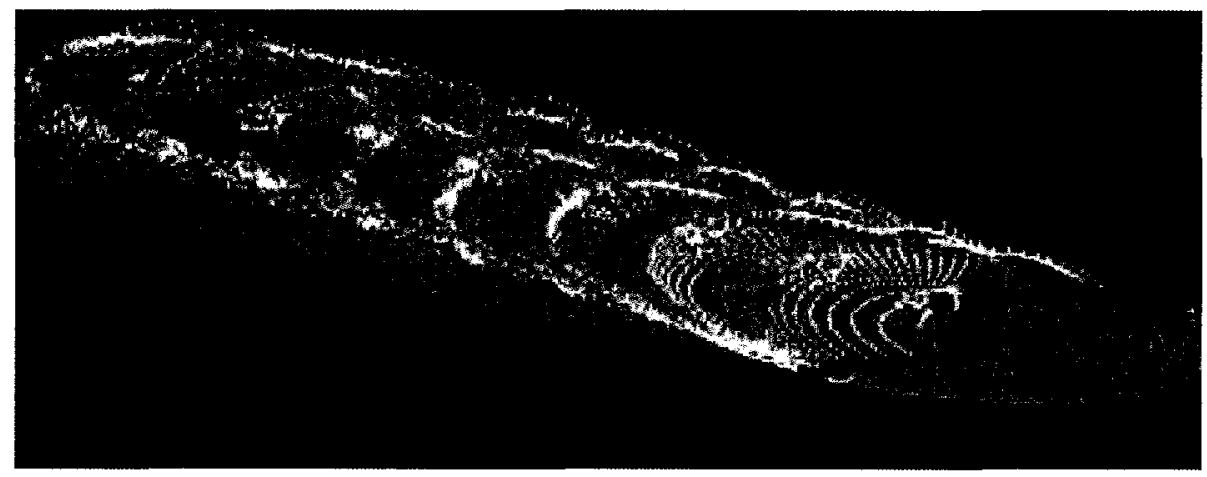

Figure 2.5 Wake produced in forward flight ${ }^{2}$.

Figures 2.4 and 2.5 show the flow structure corresponding to hover and forward flight, respectively. For the case of helicopter hovering, helicoidal flow patterns can be observed. For the case of helicopter forward flight, overlapping circular tip flow patterns are identified. As the time elapse the wake tends to dissipates by interacting with the far field flow. 
An incentive for rotor wake studies is its influence on rotor performance, structural vibration, aeroelastic stability, blade aerodynamic loads, and blade control loads. In spite of consistent progress, an advanced design for maximizing rotorcraft performance is still obstructed by the difficulty of accurately predicting the rotor wake structure and trajectory. The capability to modify the wake structure is constrained by the level of understanding of the phenomena of rotor wake formation and evolution. Unfortunately the physics behind this phenomenon of formation and evolution is still incompletely understood.

The variations with vortex age, of vortex core parameters, such as radius and swirl velocity, as well as vortex trajectory are significant factors to be considered in an accurate model of the tip vortex. The difficulties raised by the uncertain identification of the main features of the vortex core, lead to an exaggerated emphasis on vortex core parameters, defined by using the simplistic Rankine's vortex model. ${ }^{2}$ A mechanism able to explain the persistence of the tip vortices for a few rotor revolutions is still lacking as well. Significant progress was achieved, nevertheless, in both the theoretical and experimental areas. Experimental investigations showed that the blade tip geometry influences the vortex growth rate and the vortex persistence. ${ }^{3-7}$ Also experimental studies regarding the tip vortex mechanisms of formation and evolution in forward flight at a 0.1 advance ratio, showed that the blade surface roughness strongly influences the vortex characteristics. ${ }^{12}$ These observations indicate that the mechanism of flow separation, which determines the tip vortex formation, plays a significant role in the subsequent evolution and dissipation of the vortex.

Recently, more attention has been paid to investigate the effect of streamwise (axial) velocity upon the tip vortex for the case of parallel BVI. ${ }^{8}$ In the past, the influence of the axial velocity was ignored. The usual characteristic parameter for the tip vortex was the 
circumferential (swirl) velocity. Furthermore, the vortex flow used to be considered axisymmetric. The reason for this lack of three-dimensional representation is a debate in the research community regarding the significance of the core axial velocity in the relationship to the swirl velocity values. ${ }^{9}$ The investigations regarding airplane trailing vortices revealed that the velocity profile relative to the atmosphere can be found to be directed in some circumstances towards the wing ("wake-like" profile) while in other circumstances away from it ("jet-like" profile) raising further uncertainties regarding the axial velocity effect." However, for the helicopter case, measurements for a two bladed rotor in hover, showed a strong wake-like axial velocity distribution. ${ }^{10}$ This behaviour was consistently confirmed by recent experimental results in hover and in low speed forward flight. ${ }^{11-14}$

Another debating issue is related to the role played by turbulence in the mechanism of formation, evolution, and dissipation of the tip vortices. ${ }^{15}$ The experiments using the flow pattern representation have shown that growth rate and the persistence of the tip vortices for several rotor revolutions can be explained by laminar flow process rather than a turbulent one. This behaviour can be explained by the fact that the centrifugal force, due to swirling motion in the vortex core can have a stabilizing effect, impending the transport of turbulent energy from small scale eddies towards the vortex core. ${ }^{16}$ Therefore the vortex core can be materialized as a solid-body like rotation region in which the flow is mainly laminar, while the dissipation occurs at the molecular level. The complexity of a three-dimensional flow as well as the significance and source of turbulence are still subjects of fundamental research for both fixed and rotary wing.

The complexity of the unsteady rotor flow represents a high challenge for current state-of-art CFD, especially when wake capturing is involved. The limitations imposed by 
the grid resolution and numerical dissipation of the scheme generate a non-physical dissipation of the rotor wake, which reduces the prediction accuracy of the tip vortex characteristics for large tip vortex age.

Beside these CFD limitations, the uncertainties of the experimental results make it difficult to surpass the shortcomings of the turbulence models or to impose more accurately the boundary conditions in the flow solver. ${ }^{17}$ One advantage of CFD analysis comparing with the experimental investigation is the capability of capturing the data in the entire surrounding flow. Consequently, flow patterns can be captured in any region of the flow, by this providing a better understanding regarding the evolution of the tip vortex. A better understanding of the physics of the tip vortex formation will lead to a pertinent technical solution regarding the improvement of helicopter performance and reduction of the BVI effect. Experimental and numerical studies have indicated that the most significant factors in BVI noise generation are: the blade-vortex miss-distance, $\mathrm{h}$ (in the present work), defined as the separation distance of the vortex from the airfoil blade and vortex characteristics. ${ }^{18-19}$

In the past decades, many concepts regarding the reduction of BVI effects have been proposed and investigated. All proposed concepts attempt to either modify the core structure of tip vortices or to alter their trajectories. Many blade tips (ogee, vane, etc.) or special devices (spoilers, subwings, end-plates, etc.), were designed to diffuse the blade tip vortex structure in an attempt to modify the tip vortex structure to obtain a BVI noise reduction. ${ }^{19-20}$ However, it has been proved not to be successful without severe penalty in performance. Alternatively, active techniques were proposed such as using positive mass blowing jets, near the blade tip. ${ }^{21-22}$ 
Series of experimental and numerical investigations have appeared in the literature. Experimental investigations focused on the modification of the near field structure of the tip vortex. In this sense a TAMI (Tip Air Mass Injection) system was designed in order to reduce the noise output during blade-vortex interaction in descending low speed flight. ${ }^{16}$ The experimental results showed that using a spanwise TAMI system, a decrease of drag generated by induced vortex can be obtained. Another proposed way to destabilize the tip vortex structure is by using discrete blade tip jets. Similar experimental investigation showed that the core of the tip vortex is less concentrated and that the coherence of the vortex strength and core near the blade tip is reduced. ${ }^{17}$

Another very recent experimental investigation examined the modification of tip vortex core using a slatted blade tip, which incorporates four channels, distributed from the leading edge upper surface to the tip side edge. The experimental results showed that, there is a reduction of the swirl velocity peak value up to $60 \%$ relative to base line value for the case of tip vortex. ${ }^{13,14}$ It was also observed that the corresponding core radii, for different wake ages were three times larger than the ones measured at the base line. These results are due to the differences of the vorticity diffusion mechanism for the two cases. It suggests that the tip vortex diffusion of the slotted blade is more rapid due to enhanced flow mixing in the inner core, which at the same time prevents the laminar region from being sustained.

In the last years, researchers have focused their work in Individual Blade Control (IBC) due to the fast developments in the field of technology of smart structures. ${ }^{11}$, 12, 17-22 This provides a new degree of design flexibility for advanced composite helicopter blades. The key in the rotating frame is the ability to allow the structure to sense and react in a 
desired fashion, improving the rotor blade performance in the areas of structural vibration, acoustic signature and aero elastic stability.

There have been several approaches described in the literature to take advantage of active materials for individual blade control for IBC. One of the novel designs involves integral actuation through the use of anisotropic actuators, particularly through the use of active fibre composites (AFC) with interdigitated electrodes. Basic material characterization and a proof-of-concept model of integral twisted-actuated rotor blades has been also recently developed. ${ }^{20,21}$ The aim of this later investigation was to design and analyze beam-like structures taking into account the presence of embedded anisotropic actuators distributed through the blade in a consistent manner to optimize the blade twist capability under the action of the external stimulus provided by the IBC control system.. A two-cell advanced composite rotor blades with integral anisotropic active plies was designed and tested in wind tunnel.

In spite of extensive research regarding the investigation of blade-vortex mechanism of interaction, there are still many fundamental problems that have to be addressed. One of the problems is the influence of aeroelastic response of the blade on the blade-vortex mechanism of interaction. This problem will be addressed in the present investigation through a numerical approach, based on a fully coupled fluid-structure algorithm, for an idealized airfoil (flat plate) test case. 


\subsection{Flow past circular cylinder}

As mentioned in the previous chapters, the present analysis represents a test case of a cylinder-vortex interaction, to assess the feasibility of the turbulence model and numerical approach for the aimed investigation of airfoil-vortex interaction. The choice for the case of flow past circular cylinder was made on the fact that this is a canonical problem that has been extensively investigated and provides a large range of data. ${ }^{28-53}$

Despite the simple geometry, the flow past a circular cylinder exhibits complex flow phenomena that may involve transition to turbulence (inside the boundary layer), boundary layer separation, recirculation bubbles, and geometry-dependent vortex flows in the wake region, resulting in a multifaceted and challenging flow field. These flow phenomena are very common in many aerodynamic applications, such as aircraft at high angle of attack, flow past landing gears, etc. Although the flow past a cylinder has been extensively investigated both experimentally and numerically, for the past decades, viscous studies of a vortex interacting with cylindrical or prismatic structures are relatively scarce. The motivation for the present investigation is driven by the desire for a better understanding of the canonical airfoil-vortex interaction problem, which is of critical importance in rotorcraft aerodynamics.

Direct Numerical simulation (DNS) of flows at large Reynolds number is impractical due to the heavy demands on computational resources. On the other hand, it was observed that computations using Reynolds averaged equations pose great difficulty in predicting the mean forces on the cylinder and the near-wake mean flow statistics. Large-eddy simulation (LES) in conjunction with a suitable turbulence model is a promising approach. 
Flow past circular cylinder at Reynolds number $\mathrm{Re}=3900$ has become a canonical test case for large-eddy simulation (LES). ${ }^{37-39}$ Most numerical simulations have been performed at low subcritical Reynolds number range where the attached boundary layer is laminar and sufficiently thick to be easily resolved by computational grid and therefore the subgrid scale (SGS) stresses of LES are relatively low. ${ }^{39}$ The mean velocity and Reynolds stress profiles from these three simulations are in good agreement with the experimental data. However, there are some issues regarding the power spectra and the mean velocity in the recirculation region. The use of upwind scheme generated significant numerical dissipation resulting in over damping in the velocity power spectra and high wave numbers. ${ }^{40}$ This issue was overcome by using a central-difference scheme. The power spectra showed more energetic turbulence scales than those computed using upwind schemes and the simulated spectra are in good agreement with the experimental data over a wider range of wave numbers. However, no clear inertial range is obtained in either of these two simulations. The use of higher-order method based on B-spline and zonal grids allowed to accurately capturing the inertial range.$^{41}$ Although the mean velocity profiles from all three simulations agree with one another, in the vicinity of the cylinder they all differ in shape from the experimental velocity profile. Another investigation showed the numerical and modeling aspects of LES for flow past a circular cylinder at Reynolds number $\mathrm{Re}=3900$, using five different numerical schemes and two subgrid-scale models. ${ }^{36}$ The numerical results using central difference schemes were in better agreement with the experimental data than dissipative methods, while low-order upwind schemes failed to correctly predict several important physical quantities. Other investigations (regarding the effect of the averaging time on the 
accuracy of statistical quantities in LES) showed a significant influence of the accumulation time on the values of the base pressure coefficient and the recirculation bubble length. ${ }^{42}$

Several 2-D and 3-D large-eddy simulation investigations have been performed in the past few years for flow past circular cylinder for high Reynolds numbers. Kelvin-Helmholtz instabilities affect the shear layer separation from the upper and lower surface of the cylinder (for $\operatorname{Re}=8000$, upwind finite difference scheme, and dynamic subgrid scale model). ${ }^{43-46}$ However, the reported lift coefficient was much lower than the experimental values. Also the short time history collected compromised the reliability of the power spectra and the accuracy of the Strouhal frequency. Related work concluded that the origin of the shear-layer instability, which leads to mixing layer eddies, is predominantly a two-dimensional phenomenon. ${ }^{51-53}$ It was observed that the shear layer fluctuations are intermittent and become stronger with the increase of Reynolds number.

Relatively recent investigations were performed using LES for flow past circular cylinder at Reynolds number $\operatorname{Re}=140,000$ using a second-order central difference for spatial derivatives and Runge-Kutta method for time marching, with and without SGS models, in order to evaluate the applicability of LES to practical high Reynolds number flows. ${ }^{51}$ It was observed that both, the Smagorinsky and the dynamic SGS models provide results that agree fairly well with the experimental data in velocity distributions and streamwise Reynolds stresses. It was also shown that changing the Smagorinsky constant $\mathrm{C}_{\mathrm{s}}$ can affect the solution. The numerical investigation using the Smagorinsky constant value $C_{s}=0.1$ provided better results than $\mathrm{C}_{\mathrm{s}}=0.065$. However, based on the results in terms of the streamwise and normal components of the velocity, it is difficult to identify which SGS model (Smagorinsky or dynamic) performs better. In this study the cross-stream Reynolds stresses are over predicted 
by both SGS models. Recent LES study for flow past cylinder at $\mathrm{Re}=10,000$ was performed using a stabilized finite element formulation and Smagorinsky subgrid scale (SGS) model. ${ }^{52}$ The predicted drag coefficient and Strouhal number are in fairly good agreement with the experimental data.

With the improvements in computational power, numerical simulation of flow past circular cylinder were performed for $\mathrm{Re}=10^{6}$ using LES, with and without the Smagorinsky subgrid scale (SGS) model. ${ }^{53}$ No difference between the two test cases was reported. Higher Reynolds numbers (supercritical) from $R e=5 \times 10^{5}$ to $2 \times 10^{6}$ have been recently investigated using LES with a wall model. ${ }^{53}$ The mean pressure distribution is fairly well predicted at lower Reynolds numbers, while the results are inaccurate at the higher Reynolds numbers. It has been demonstrated that three-dimensionality in the wake leads to irregular states and fast transitions to turbulence at Reynolds numbers just beyond the onset the secondary instability. The three-dimensionality effect on the accuracy of simulations has been investigated for $\operatorname{Re}=$ 500 and $\operatorname{Re}=525$ through a number of 2-D and 3-D direct numerical simulations (DNS). ${ }^{42}$ It was found that the over prediction of fluid forces in 2-D simulations is primarily a 3-D effect.

\subsection{Aeroelasticity}

Aeroelasticity is a discipline where the mutual interaction between aerodynamic forces and elastic forces on lifting surfaces is investigated. From the various areas where aeroelastic phenomena concern designers, aircraft and rotorcraft are the most important, but also in propellers, turbines, compressors, wind turbines, and wind-induced loads on structures (e.g. suspension bridges etc.) aeroelastic phenomena are always present. If these structures were 
perfectly rigid, aeroelastic problems would not exist. But aeroelastic phenomena arise when structural deformations induce additional aerodynamic forces, which in turn may produce additional structural deformations, which again will induce still greater aerodynamic forces. Such interactions may tend to become smaller and smaller until a condition of stable equilibrium is reached, or it may tend to diverge and destroy the structure.

Flutter is of particular concern in the design of helicopter blades. Unstable vibrations may arise due to coupling between the aerodynamics and the structural dynamics. If the fluid flow produces positive net work on a vibrating blade so as to amplify or auto-sustain the vibration, then the blade is said to be undergoing flutter. The ability to understand and predict this phenomenon is crucial to ensuring that the helicopter rotor operates within stability boundaries and thus has a large impact on the design process. Appropriate blade design, together with strategies for controlling the onset of instabilities can significantly impact the stable operating range potentially leading to better helicopter performance.

On the other hand, dynamic forced response of the helicopter blade to various inputs such as blade-vortex interaction (BVI) is an important factor in determining the stress loads on the blades and vibrations transmitted to the hub. In particular, periodic forcing inputs such as that due to BVI may induce a large blade response if the frequency of excitation is near the blade natural frequency. Blade forced response vibrations can lead to high cycle fatigue which can in turn cause blade failure. Accurate prediction of blade response to external inputs can facilitate improved understanding of forced response phenomena allowing design strategies to be adopted to minimise their impact and potentially prolong life of helicopter components. Consideration of aeroelastic effects is vital at the design stage to ensure that the helicopter blade will operate within an acceptable response region. 
Aeroelastic phenomena involve a complicated interaction between the aerodynamics and the structural dynamics of the blades. The challenge is to develop a model which accurately captures the relevant dynamics of both the fluid and the structure and more importantly the interactions between the two.

Since most structures are near-linear in deformation with increasing load, the structures are modeled as linear oscillators. If only a single structural degree of freedom is excited, the structural motion is described by a single linear equation with fluid forcing. If more then one degree of structural freedom is present, linear systems of equations are required. Fluid models are more difficult. Fluid mechanics is an inherently nonlinear, multidegree-of-freedom phenomenon.

Extensive numerical investigations have been performed based on both linear and nonlinear theory. ${ }^{58-68}$ It has been shown in structural dynamics that linear theory offers poor response predictions at high sound pressure levels (SPL) ${ }^{58-60}$ In order to overcome this issue several nonlinear models were proposed. ${ }^{61,62}$ However, it is important to notice that in all the above studies, the fluid-structure coupling was neglected.

Relatively recent studies have shown that indeed there is a strong coupling between structure vibration and the surrounding flow field that should be considered. ${ }^{64-68}$

More recent studies have emphasized the importance of accounting for the fluid-wall shear stresses on the nonlinear structural response. ${ }^{67,68}$ It was found that under certain conditions, the fluid-wall shear stresses reduce the level of structural vibration and change the response from nonlinear to linear.

The literature review revealed the fact that a comprehensive analysis of airfoil/bladevortex interaction is lacking at the moment. Also the aeroelastic response of an airfoil to 
airfoil-vortex interaction using a strong coupling has not been investigated. On the other hand in spite of the extensive research regarding flow past circular cylinder, the vortex-cylinder interaction has never been investigated.

Based on theses facts, the present research proposes a comprehensive analysis regarding the investigation of airfoil-vortex interaction, vortex-cylinder interactions and the aeroelastic response of an idealized airfoil to airfoil-vortex interaction. The present research represents a pioneering work regarding the numerical investigation of airfoil-vortex mechanism of interaction using large-eddy simulation. ${ }^{70-73}$ 


\section{Chapter 3: Numerical Methods for AVI and Turbulent Flows}

The numerical simulation of AVI presents significant challenges since most numerical techniques either tend to alter the characteristics of the vortex before the interaction occurs or are not able to capture all the fluctuations of the aerodynamic coefficients. The presently available solution techniques for predicting the unsteady aerodynamic responses of rotor blades during BVI can be classified in three main categories: 1) Lagrangian vortex methods (considered to be beyond the scope of the present work and not discussed in detail here), 2) inviscid panel methods with rigidly prescribed wake geometry or with free wake geometry, and 3) methods using Euler or Navier-Stokes equations.

Inviscid panel methods have limitations when fluid flow separation is concerned. The Navier-Stokes equations can be solved directly (DNS, Direct Numerical Simulation) without modeling. However, due to a wide range of length and time scales present in turbulent flows, DNS is still restricted to low-Reynolds number flows in relatively simple geometries. DNS of high-Reynolds number flows of practical interest would necessitate tremendous resolution requirements that are far beyond the reach of the capability of even the fastest supercomputers available today.

Classical Computational Fluid Dynamics (CFD) tools using Reynolds Averaged Navier-Stokes (RANS) equations, such as URANS (unsteady RANS) have proven to be highly dissipative and may not explore unsteady flows accurately. RANS methods heavily rely on the turbulence models to model all relevant scales of turbulence. Moreover, such methods try to predict the noise using the mean flow properties provided by a RANS solver. Since noise generation is a multi-scale problem which involves a wide range of length and 
time scales, it appears that the use of RANS-based prediction methods will remain limited, unless advanced turbulence models capable of accurately modeling a wide range of turbulence scales are developed and implemented into existing RANS solvers. An alternative approach aiming at overcoming the issue associated with the modeling dissipation is largeeddy simulation (LES), which in spite of the high computational cost, preserves the characteristics of the vortex and provides instantaneous values of the variables. LES, with lower computational cost, is a promising alternative to DNS, being probably the most powerful computational tool to be used, since it is the only way, other than DNS, to obtain a time-accurate unsteady solution.

\subsection{Direct Numerical Simulation}

Direct Numerical Simulations (DNS), as the name suggest is a straight forward approach to the simulation of turbulent flows. DNS is the most accurate but expensive approach. When using the DNS approach, all the scales of motion are simulated by solving the Navier-Stokes equations. No averaging or approximation is undertaken to solve the governing equations. In the case of incompressible fluids with uniform density, the non-dimensionalized NavierStokes equations are:

$$
\begin{aligned}
& \frac{\partial u_{i}}{\partial x_{i}}=0 \\
& \frac{\partial u_{i}}{\partial t}+\frac{\partial}{\partial x_{j}}\left(u_{i} u_{j}\right)=-\frac{\partial P}{\partial x_{i}}+\frac{1}{R_{e}} \frac{\partial^{2}}{\partial x_{j}^{2}} u_{i}
\end{aligned}
$$


where the standard tensor notation of repeated indices represents the summation, $\mathrm{P}=p / \rho$ and $\operatorname{Re}=U L / v$ where $U$ represents the characteristic velocity, $L$ represents the characteristic length and $v$ represents the kinematic viscosity.

In order to capture the significant structures, the length scale of the domain has to be at least as large as the largest turbulent eddy. A possible measure would be the integral scale $(L)$ of the turbulence which is the distance over which the velocity remains correlated (i.e. it assumes that the velocities within an eddy are highly correlated, whereas there is no correlation between velocities in different eddies). Theoretically, the largest eddy goes to infinity, therefore the characteristic turbulent length representing the mean of all existing eddies is applied, which is defined as

$$
L_{i}=\frac{1}{\overline{u_{i}^{\prime} u_{j}^{\prime}}} \int_{0}^{\infty} R_{i j} d r_{i}
$$

where $R_{i j}$ is the correlation tensor of velocity components

$$
\mathrm{R}_{i j}(x, t, r, \tau)=\overline{u_{i}^{\prime}(x, t) u_{j}^{\prime}(x+r, t+\tau)},
$$

where $x+r$ is a neighbouring point with the distance $r$ to $x$. Turbulent flows are dominated by eddies (vortices) and correlation represent a useful tool to analyze this phenomenon.

The simulation must capture all the kinetic energy dissipation, which occurs on the smallest scales having the lowest energy. The smallest length and time scale present in turbulent flows are called respectively the Kolmogorov length and Kolmogorov time scale and it can be determined by the dissipation rate $\varepsilon$, representing the transformation from kinetic energy into thermal energy and the kinematic viscosity, $v$, where, $\eta=\left(v^{3} / \varepsilon\right)^{1 / 4}$ and $\tau=(v / \varepsilon)^{1 / 2}$. The inconvenience of this approach is that in order to successfully solve the Navier-Stokes equations, the computational domain has to be large enough to contain the 
largest scales $L$, of motion, while the grid resolution has to fine enough in order to resolve the smallest scales, which are of the order of Kolmogorov micro scale $\eta=\left(v^{3} / \varepsilon\right)^{1 / 4}$, where $\eta$ is the dissipation rate per unit mass and $v$ is the kinematics viscosity. Hence, the number of grid points necessary in each direction can be estimated as $L / \eta$ and using the relationship between $L / \eta$ and the Reynolds number Re, we have that $N=(L / \eta)^{3} \approx \operatorname{Re}^{9 / 4}$, where $N$ is the number of grid points. From this relation it can be seen that the DNS approach is practical only for low Reynolds numbers. Even though DNS is not feasible for most turbulent flow simulations, it is a useful tool to validate turbulence models.

Most approaches to the numerical simulations of high-Reynolds numbers flows are based on the insight gained from the physical description of turbulence. The irregularity is one of the most important characteristics of the turbulent flows. Although it is difficult to completely describe the turbulence as a function of time and space coordinates, it is possible to describe the average values of the flow velocity (pressure and velocity). Rigorous observations of turbulent flows showed that these averages exist because:

1. At any given point in the turbulent domain a distinct pattern is repeated more or less regularly in time.

2. At any given point in the turbulent domain, a distinct pattern is repeated more or less regularly in space; hence roughly speaking turbulence has the same overall structure into the considered domain. 
Moreover, the details of the motion at the level of small scales are not of interest for most of the engineering applications. Osborne Reynolds was the first one that in 1895 developed a statistical approach and derived the equations that are called after his name, to describe the dynamics of "mean" (average) flow.

\subsection{Reynolds Averaged Navier-Stokes Simulation (RANS)}

Opposite to the DNS is the Reynolds Averaged Navier-Stokes Simulations (RANS). In the case of RANS simulations the flow (velocity field and pressure for incompressible flows) is divided into a statistically steady (mean) portion and fluctuations. By this approach the mean flow is solved and the fluctuations are modeled. By modeling the fluctuation effects, RANS models all turbulence interactions whereas the DNS completely resolve them. The inconvenience of the RANS is that there is no model for the fluctuation effects that could be used for the turbulent flows.

\subsubsection{The Shear Stress Transport Model (SST)}

In the present work, the Reynolds Averaged Navier-Stokes equations (RANS) were solved using the CFX-11.0 (ANSYS Inc.) software. To solve for the turbulent stresses in the RANS equations, the Shear Stress Transport (SST) turbulence model was used. The SST model combines the existing $k-\varepsilon$ and $k-\omega$ models. It is found that the $k-\omega$ model is too sensitive to the specification of inlet boundary conditions for $\omega$, while this issue is not encountered by the 
$\mathrm{k}-\varepsilon$ model. Thus the k- $\varepsilon$ is generally preferable, but the $\mathrm{k}-\omega$ is better in the sublayer of the boundary-layer. The k- $\varepsilon$ equations are written in terms of $\omega$. The result is:

$$
\begin{aligned}
& \frac{\partial k}{\partial t}+U_{i} \frac{\partial k}{\partial x_{i}}=\tau_{i j} \frac{\partial u_{i}}{\partial x_{j}}-\beta k \omega+\frac{\partial}{\partial x_{i}}\left[\left(v+\sigma v_{e}\right) \frac{\partial k}{\partial x_{i}}\right] \\
& \frac{\partial \omega}{\partial t}+U_{i} \frac{\partial \omega}{\partial x_{i}}=\alpha \frac{\omega}{k} \tau_{i j} \frac{\partial u_{i}}{\partial x_{j}}-\beta^{\prime} \omega^{2}+\frac{\partial}{\partial x_{i}}\left[\left(v+\sigma^{\prime} v_{e}\right) \frac{\partial \omega}{\partial x_{i}}\right]+2(1-F) \frac{\sigma^{\prime}}{\omega} \frac{\partial k}{\partial x_{i}} \frac{\partial \omega}{\partial x_{i}} \\
& F=\operatorname{Tanh}\left(\arg ^{2}\right) \\
& \arg =\max \left[2 \frac{\sqrt{k}}{0.09 \omega y}, \frac{500 v}{\omega y^{2}}\right]
\end{aligned}
$$

The distance to the closest wall is $\mathrm{y}$. The constants for the $\mathrm{k}-\omega$ model are:

$\beta=0.09, \alpha=5 / 9, \beta^{\prime}=3 / 40, \sigma=0.5, \sigma^{\prime}=0.5$

Constants for the k- $\varepsilon$ model are:

$$
\beta=0.09, \alpha=0.44, \beta^{\prime}=0.0828, \sigma=1, \sigma^{\prime}=0.856
$$

These k- $\varepsilon$ and k- $\omega$ models are now combined, by blending. Let $\Phi$ represent one of the above constants, and a subscript of 1 refer to the $k-\omega$ model and 2 refer to the $k-\varepsilon$ model. The actual constant is determined by:

$$
\Phi=F \Phi_{1}+(1-F) \Phi_{2}
$$

Menter makes a further change by limiting the eddy viscosity by a new formula for the eddy viscosity:

$$
v_{e}=\frac{0.31 k}{\max (0.31 \omega, \Omega F)}
$$

The term $\Omega$ is the absolute value of vorticity. 


\subsection{Large-Eddy Simulation (LES)}

A third numerical approach for the computation of the turbulent flows is large-eddy simulations (LES), which is an intermediary approach between DNS and RANS. The motivation for LES is that since the large energy carrying eddies are highly influenced by the boundary conditions they can be computationally resolved while the small eddies or unresolved scales are modeled. In general the large scales of motions are more energetic than the small ones and their size and strength make them the most effective transporters of the conserved properties. Therefore an approach which treats the large eddies more accurately than the small ones would be more suitable. Large-eddy simulation is the approach that would meet this requirement.

Large-eddy simulations are three-dimensional, time dependent and expensive, but less costly than DNS. Large-eddy simulation is a suitable approach for the case of high Reynolds number or when the geometry is too complex for the DNS approach. As was presented in the previous section, large-eddy simulation is a result of space averaging operation applied to Navier-Stokes equations. The mathematically closed form is obtained by a modeling technique using the Fourier space. The principle of large-eddy simulation is to separate the large eddies which are computationally resolved from the small eddies which are modeled. Large-eddy simulation, roughly speaking, is a method that resolve only those turbulent structures (eddies) that can be captured by a certain grid size. All of the smaller eddies are modeled. The advantage of this method is that it provides much more information than statistical methods and it does not require so much computational time as DNS does. From a point of view of computational expense large-eddy simulation lies between Reynolds-stress 
models and DNS and presents interests due to the limitations of the other approaches. There are four conceptual steps in LES:

1. A filtering operation has to be defined in order to decompose the velocity $u(x, t)$ into a sum of a filtered (resolved) component $\bar{u}(x, t)$ and a residual (subgrid-scale, SGS) component $u^{\prime}(x, t)$.The filtered velocity field $\vec{u}(x, t)$, which is three-dimensional and time-dependent, represents the motion of the large eddies.

2. The equations for the evolution of the filtered velocity field are derived from the Navier-Stokes equations. These equations are similar to the Navier-Stokes equations in the standard form except that the momentum equation contains the residual stress tensor (SGS stress tensor) that arises from the residual motions.

3. Closure is obtained by modeling the residual stress tensor, by an eddy viscosity model (the easiest way possible).

4. The model filtered equations are then solved numerically for $\vec{u}(x, t)$, which provides an approximation to the large scale motions in one realization of the turbulent flow.

It is very important to define in a precise way the quantities that will be computed. It is necessary a velocity field that contains only the large scale components of the total field. Leonard in 1974 showed that this can be done by a filtering; the large scale or the resolved scale is the one to be simulated and represents the local average of the complete field. In his work, Leonard defined a function $f(x, t)$ which contains all the scales. Let $f(x, t)$ be an instantaneous flow variable (velocity or pressure) and G denote an averaging kernel, which is a localized function.

More precisely: 
$G(0)>0, \int_{\mathfrak{R}^{d}} h(x) d x=1$ with $h(x) \rightarrow 0$ rapidly, as $|x| \rightarrow \infty$

The filter of $\mathrm{f}$ denoted as $\bar{f}$, is defined as the convolution of $\mathrm{f}$ with a filter function let say $\mathrm{G}(\mathrm{x}) \bar{f}(x)=\int_{\Omega} G(x-s) f(s) d s$, where $\Omega$ is the flow volume is normalized so that

$$
\int_{\Omega} G(s) d s=1
$$

and

$G(-x)=G(x)$

The condition $G(-x)=G(x)$, ensures that when $\mathrm{f}$ is constant $\bar{f}=f$.

\subsubsection{Governing Equations}

Assuming that the error in the interchange of filtering and differentiation can be ignored (i.e. $\overline{\partial f} / \partial x=\partial \bar{f} / \partial x)$ the filtered Navier-Stokes equations are :

$$
\begin{aligned}
& \frac{\partial \overline{u_{i}}}{\partial x_{i}}=0 \\
& \frac{\partial \overline{u_{i}}}{\partial t}+\frac{\partial}{\partial x_{j}}\left(\overline{u_{i} u_{j}}\right)=-\frac{\partial \bar{P}}{\partial x_{i}}+\frac{1}{R_{e}} \frac{\partial^{2}}{\partial x_{j}^{2}} \overline{u_{i}}
\end{aligned}
$$

By filtering the above equations, two unknowns were introduced but no additional equation was introduced so the system is not mathematically closed. The system of equations is undetermined.

This is known as the famous closure problem and it can be stated as: writing the velocity as $u=\bar{u}+u^{\prime}, \overline{u u}=\overline{\overline{u u}}+\overline{\bar{u} u^{\prime}}+\overline{u^{\prime} \bar{u}}+\overline{u^{\prime} u^{\prime}}$, representing the decomposition of the non- 
linear interactions, where $\bar{u}$ is the large component of $u$ and $u^{\prime}$ is the small or subgrid scale. In order to mathematically close the system, the LES has to model $\bar{u}$ in terms of $\bar{u}$ only. From the definition of filtering $\bar{f}(x)=\int_{\Omega} G(x-s) f(s) d s$,

it can be seen that in general $\overline{\bar{u}} \neq \bar{u}$ unless $u$ is constant or the filter is ideal low pass filter. This means that in general $\overline{u^{\prime}} \neq 0$. In the Reynolds averaging method, the decomposition is in terms of the statistical mean and the fluctuations are with 0 mean. In order to overcome the closure problem, the filtered Navier-Stokes equations are rewritten in the following form:

$$
\begin{aligned}
& \frac{\partial \overline{u_{i}}}{\partial x_{i}}=0 \\
& \frac{\partial \overline{u_{i}}}{\partial t}+\frac{\partial}{\partial x_{j}}\left(\overline{u_{i} u_{j}}\right)=-\frac{\partial \bar{P}}{\partial x_{i}}-\frac{\partial}{\partial x_{j}} \tau_{i j}+\frac{1}{R_{e}} \frac{\partial^{2}}{\partial x_{j}^{2}} \overline{u_{i}} \\
& \text { where } \tau_{i j}=\overline{u_{i} u_{j}}-\overline{u_{i} \bar{u}_{j}}
\end{aligned}
$$

is called the subgrid scale (SGS) Reynolds stress. The SGS Reynolds stress is similar to the Reynolds stress in RANS in the sense that in both cases the unresolved scales $u^{\prime}$ ( small eddies in LES and turbulent fluctuations in RANS) are regarded as producing stresses in the resolved scales (large eddies in LES and the mean velocity in RANS).

The difference is that in RANS, $u^{\prime}$ represents all the turbulent motions, while in LES $u^{\prime}$ represents only the small eddies or subgrid scales. This means that the energy in the unresolved scales of LES (subgrid scales) represents a much smaller fraction of the total flow energy comparing with the energy in the unresolved scales of RANS (the turbulent fluctuations). There are two main approaches to solve the closure problem. A first approach is 
the one referred to as the Lilly-Deardroff approach. ${ }^{30}$ This approach permits to define the filtering operator implicitly through the modeling of the SGS stress term. A second approach is referred to as the Leonard approach, which is considering the substitution of $u_{i}=\overline{u_{i}}+u_{i}^{\prime}$ into $\overline{u_{i} u_{j}}$ and obtaining $\overline{u_{i} u_{j}}=\overline{u_{i} u_{j}}+\overline{u_{i}^{\prime} \overline{u_{j}}}+\overline{\overline{u_{i} u_{j}^{\prime}}}+\overline{u_{i}^{\prime} u_{j}^{\prime}}$. It is important to notice that the first term in the right hand side of the equation can be explicitly calculated if the filter used is itself explicitly defined. Using the above result the SGS stress term in equation (3.20) becomes

$$
\tau_{i j}=\left(\overline{\overline{u_{i} u_{j}}}-\overline{u_{i}} \overline{u_{j}}\right)+\left(\overline{\left(\overline{u_{i}^{\prime}} \overline{u_{j}}\right.}+\overline{\overline{u_{i}} u_{j}^{\prime}}\right)+\overline{u_{i}^{\prime} u_{j}^{\prime}}
$$

The great advantage of this approach is that splits the SGS stress into three terms that have a physical interpretation in the flow. The first term in the right hand side of the equation (3.31) represents the interaction of the large eddies that produce small eddy effects and it is called the Leonard stress. The second term represents the interaction between large and small eddies and is called the cross term. The third term represents the interaction of small eddies to produce large eddy effect. This term is the one that generates the transfer of energy from the small to the large eddies and it is known as the back scatter term. The second term may also produce back scatter. The inconvenience of the Leonard approach is that the filter must be explicitly known to be able to calculate the Leonard stress term. This is a quite difficult issue, especially in the grid based methods where the filter is often implicitly defined. 


\subsubsection{Subgrid scale Modeling (SGS)}

As it has been shown in the previous section, a new term was introduced in the filtered Navier-Stokes equations, which will lead to the mathematical closure of the problem. In order to solve this problem, the filtered Navier-Stokes equations are rewritten in the form given by equation (3.32) and the new term $\tau_{i j}$ is then modelled. In this section two of the most common models are presented, namely the Smagorinsky and the dynamic subgrid scale models. Also a variant of the Smagorinsky model will be presented and named as modified Smagorinsky model.

\subsubsection{Smagorinsky Model}

Smagorinsky proposed in 1963 , the most commonly used model. ${ }^{26}$ This model is an eddy viscosity model based on the fact that the effects of the SGS Reynolds stress are the increased transport and dissipation. These are the effects of the viscosity in laminar flows.

The Smagorinsky subgrid scale model is given by the equation:

$$
\tau_{i j}-\frac{1}{3} \tau_{k k} \delta_{i j}=-v_{T}\left(\frac{\partial \overline{u_{i}}}{\partial x_{j}}+\frac{\partial \overline{u_{j}}}{\partial x_{i}}\right)=-2 v_{T} \overline{S_{i j}}
$$

where $v_{T}$ is called eddy viscosity and :

$$
\overline{S_{i j}}=\frac{1}{2}\left(\frac{\partial \overline{u_{i}}}{\partial x_{j}}+\frac{\partial \overline{u_{j}}}{\partial x_{i}}\right) \text {. }
$$

This model is known as an eddy viscosity type model because it assumes that the small eddies remove energy from the flow by a dissipative process. This leaves open the possibility 
of back scatter (the reintroduction of energy from the small to large eddies) which is a weak point of the model. According to the turbulence theory, energy is introduced at the largest scales and is transferred to the smallest scales until the viscous damping becomes the dominant effect. Therefore in the regions where the viscous effect is dominant, the turbulent energy of the flow is damped out by the transfer of the kinetic energy into internal energy. Between these two subscales there is a region where there is neither significant production nor dissipation of energy, known as inertial sub-range. In this region the only active mechanism is the inviscid one and it is assumed that the transfer of energy occurs from the large scales to the small ones. The term responsible for this transfer of energy is the advection term in the Navier-Stokes equation. This allows evaluating the rate of energy transfer to the small scales. The magnitude of the contribution of the advection term to the kinetic energy is given by:

$\frac{1}{2} \frac{\partial}{\partial x_{j}}\left(u_{i} u_{i} u_{j}\right)$.

Since the energy is introduced at the largest scales, the rate of dissipation $\varepsilon$ can be estimated as: $\varepsilon=\frac{U_{L}^{3}}{L}$,

where $U_{L}$ is the velocity scale of the large eddies and $\mathrm{L}$ is the integral length scale of the turbulent flow. If it is assumed that the largest subgrid scales are much larger than the viscous scales, then:

$\varepsilon \approx \frac{U_{S G S}^{3}}{\Delta}$ 
where $U_{S G S}$ is the velocity scale of the small eddies and $\Delta$ is the length of the largest sub-grid scale eddies, which is also the length scale associated with the filter.

Based on the turbulence theory, which states the energy is transferred from the large scales to small scales, it means that once the energy is lost by the large eddies to the small eddies, it cannot be restored. SGS represents this energy transfer as an effective viscous dissipation. Because the smallest resolved scales (of size $\Delta$ ) are most significant for the SGS model, the effective viscous dissipation can be estimated as:

$\varepsilon \approx \frac{v_{T} U_{S G S}^{2}}{\Delta^{2}}$

Replacing $\varepsilon$ from equation (3.26) into equation (3.27) results that $v_{T} \propto \Delta U_{S G S}$ and using the previous results, another relationship can be developed as:

$v_{T} \approx \Delta^{\frac{4}{3}} L^{-\frac{1}{3}} U_{L}$

Making use of the relation

$U_{L} \approx L\left(2 \overline{S_{i j} S_{i j}}\right)^{\frac{1}{2}}=L|\bar{S}|$,

with the property

$\left(\overline{S_{i j}} \overline{S_{i j}}\right)^{\frac{1}{2}}=|\bar{S}|$,

a new expression of $v_{T}$ can be obtained

$v_{T}=C_{S}^{2} \Delta^{\frac{4}{3}} L^{\frac{2}{3}}|\bar{S}|$,

where $C_{S}$ is the Smagorinsky parameter, which is introduced in order to ensure the equality.

Theories provide estimates of the constant. Although most of these are unfortunately valid only for isotropic turbulence, the theories agree that $C_{S} \approx 0.2$. However $C_{S}$ is not a constant 
and may be a function of Reynolds number and other non-dimensional parameters and it can be different for different flow regimes. Due to the difficulty of calculating the integral length scale, $L$, most of researchers make the simplification: $\Delta^{\frac{4}{3}} L^{\frac{2}{3}} \rightarrow \Delta^{2}$, which leads to the more common form of the eddy viscosity in the Smagorinsky model:

$$
v_{T}=\left(C_{S} \Delta\right)^{2}|\bar{S}|
$$

A common choice for $\Delta$ is $\left(\Delta_{1} \Delta_{2} \Delta_{3}\right)^{\frac{1}{3}}$, where $\Delta_{i}$ is the width associated with the filter in the i-th coordinate direction. By using an appropriate length scale, the damping becomes unnecessary.

Although the Smagorinsky model is the most used one in the SGS model, it presents some weak points. One major problem is that in the case of non-homogenous wall bounded flows, near the surface of the walls. The model does not damp out the eddy viscosity enough to allow the kinematic viscosity effects to become dominant. As result, as the wall and hence the viscous sublayer is approached, the approximation made in the derivation of the model becomes questionable since the grid points and hence $\Delta$ will be in or near the viscous sublayer region. Another issue is that Smagorinsky model assumes that the transfer of energy from the large scales to small scales is a diffusive process, so there is no mechanism to transfer energy from the small scales to the large scales (i.e. there is no back scatter). The resolved transport energy equation is given by:

$$
\begin{aligned}
& \frac{\partial \bar{q}^{2}}{\partial t}+\frac{\partial\left(\bar{q}^{2} u_{j}\right)}{\partial x_{j}}=\frac{\partial}{\partial x_{j}}\left(-2 \bar{p}_{j}-2 \bar{u}_{i} \tau_{i j}+\frac{1}{\operatorname{Re}} \frac{\partial \bar{q}^{2}}{\partial x_{j}}\right)-\frac{2}{\operatorname{Re}} \frac{\partial \bar{u}_{i}}{\partial x_{j}} \frac{\partial \bar{u}_{i}}{\partial x_{j}}+2 \tau_{i j} \bar{S}_{i j} \\
& \text { where } \bar{q}^{2}=\bar{u}_{i} \bar{u}_{i}
\end{aligned}
$$

is one-half of the last term defined as 
$\varepsilon_{S G S}=\tau_{i j} \bar{S}_{i j}$

which referred to as "subgrid scale dissipation" and represents the transfer of energy between large scales and small scales.

It is important to note that when $\varepsilon_{S G S}<0$, the resolved energy decays with respect to the interaction between large and small scales (forward scatter) when $\varepsilon_{S G S}>0$, the resolved energy grows with respect to the interaction of the large and small scales (back scatter). In the case of Smagorinsky model, an equation (3.32) and (3.35) demonstrates that $\varepsilon_{S G S}<0$, hence there is no back scatter when Smagorinsky model is used.

If we wish to simulate more complex and higher Reynolds number flows, it may be important to have a more accurate model such as DNS.

\subsubsection{Modified Smagorinsky Model}

As it was mentioned in the previous sections, one of the problems of the Smagorinsky model is that the eddy viscosity does not damp out when approaching the walls. In order to solve this problem a damping function is introduced in the calculation of the Smagorinsky parameter. This model was developed by Lilly (1962). In order to define the damping function first the kinetic energy is defined as $k=\frac{1}{2} \bar{u}_{i} \bar{u}_{i}$, the instantaneous rate of dissipation as $\varepsilon=v \frac{\partial \bar{u}_{i}}{\partial x_{j}} \frac{\partial \bar{u}_{i}}{\partial x_{j}}$ and the instantaneous Reynolds number as $\operatorname{Re}_{i n s t}=\frac{k^{2}}{v \varepsilon}$. It is important to notice that expanding $\bar{u}_{i}$ in Taylor series near the wall and assuming that the wall location is 
at $\mathrm{x}=0$, then the only non-zero velocity vector will be in the wall normal direction $y$, assuming the wall to be in the $x-z$ plane. Hence

$\bar{u}_{i}=\left.\bar{u}_{i}\right|_{x=0}+\left.y \frac{\partial \bar{u}_{i}}{\partial y}\right|_{x=0}+$ higher order terms

Using the non slip boundary condition $\bar{u}_{i}(0)=0$, hence $O(k)=y^{2}$. Based on the same $\operatorname{argument} O(\varepsilon)=1$, hence $\operatorname{Re}_{\text {inst }} \approx y^{4}$.

The modified Smagorinsky model is defined in the way:

$v_{T}=\left(C_{s}^{\prime} \Delta\right)^{2}|\bar{S}|$

$C_{s}^{\prime}=C_{s}\left\{\begin{array}{l}\left(\frac{\mathrm{Re}_{\text {inst }}}{\operatorname{Re}^{o}}\right)^{\gamma} \quad \text { for } \quad 0 \leq \mathrm{Re}_{\text {inst }} \leq \mathrm{Re}^{0} \\ 1 \text { for } \mathrm{Re}_{\text {inst }} \geq \mathrm{Re}^{o}\end{array}\right.$

where $\operatorname{Re}^{0}=\mu\left[\operatorname{Re}_{\text {inst }}\right]_{\max } \cdot\left[\operatorname{Re}_{\text {inst }}\right]_{\max }$ represents the largest value of $\left[\operatorname{Re}_{\text {inst }}\right]$ in the computational domain at a given time.

\subsubsection{Dynamic Subgrid scale Model}

A new method, so called dynamic subgrid scale model was proposed to overcome the problems of the Smagorinsky model. ${ }^{29}$ The method is calculating the Smagorinsky parameter interactively during the run time rather the setting the parameter to a constant value at the beginning of the numerical calculations. The newly developed procedure is presented in this section. The procedure consists of the application of a second filter, which is called a test filter (represented by the tilde, $\sim$ ), to the filtered momentum equations (3.44). This result into 
$\frac{\partial \tilde{\bar{u}}_{i}}{\partial t}+\frac{\partial}{\partial x_{j}}\left(\tilde{\bar{u}}_{i} \tilde{\bar{u}}_{j}\right)=-\frac{\partial \tilde{\bar{P}}}{\partial x_{i}}-\frac{\partial T_{i j}}{\partial x_{j}}+\frac{1}{R_{e}} \frac{\partial^{2}}{\partial x_{j}^{2}} \tilde{\bar{u}}_{i}$

where $T_{i j}=\tilde{u_{i} u_{j}}-\tilde{\bar{u}_{i}} \tilde{\bar{u}}_{j}$.

Using the Smagorinsky model as the SGS model we obtain the filter and the test filter levels,

$\tau_{i j}-\frac{1}{3} \tau_{k k} \delta_{i j} \approx-2 C_{s} \Delta^{2}|\bar{S}| \bar{S}_{i j}=-2 C_{s} B_{i j}$

$T_{i j}-\frac{1}{3} T_{k k} \delta_{i j} \approx-2 C_{s} \Delta^{2}|\tilde{\bar{S}}| \tilde{\bar{S}}_{i j}=-2 C_{s} A_{i j}$

where $C_{s}$ was used instead of $C_{s}^{2}$ in the eddy viscosity term, and $\Delta$ and $\tilde{\Delta}$ represents the width of the first filter and the width of the test filter respectively.

The SGS stress at the filter and the test filter level are related to the resolved turbulent stress through an algebraic identity known as Germano's identity: ${ }^{29}$

$L_{i j}=T_{i j}-\tilde{\tau_{i j}}$.

It is important to notice that in the case when the models for the stresses are used, Germano's identity is no longer exactly satisfied. Hence the residual equation is obtained:

$$
E_{i j}=L_{i j}+2 C_{s} A_{i j}-2 C_{s} \tilde{B}_{i j}
$$

The method is based on minimizing the residuals, assuming that the Smagorinsky parameter $C_{s}$ is known (denoted by $C_{s}^{*}$ ) in the third term on the right side of equation (3.43). Hence the residual expression becomes:

$$
E_{i j}=L_{i j}+2 C_{s} A_{i j}-2 C_{s}^{*} \tilde{B}_{i j} .
$$

The Smagorinsky parameter $C_{s}^{*}$ is approximated by using the Taylor series expansion of $C_{s}$. 
One of the advantages of the dynamic SGS model is that there are no parameters that need to be determined before the numerical run. Also the dynamic SGS model has the capability to reduce the eddy viscosity in the laminar region of the flow. Although Piomelli's version of the dynamic SGS model was developed to solve some of the problems of Germano's original dynamic procedure, there are still some unresolved issues. One of the problems is that in the derivation of the model, the Smagorinsky parameter was assumed to be independent of the filter width. Using the DNS data from the isentropic turbulence Meneveau found that the instantaneous values of the Smagorinsky parameter varied significantly when compared for two different filter widths.

Another issue with the dynamic SGS is that it allows $C_{s}$ and hence the eddy viscosity to be negative. Even though this would allow the model to account for back scatter, the eddy viscosity remains negative long enough, leading to numerical instabilities. As a summary the double filtering remains an open question. In the derivation of the dynamic SGS model, it is assumed that filtering twice is equivalent to filtering once with a specific filter.

\subsubsection{Aeroacoustic modeling using large-eddy simulation}

The noise generated from helicopters can be classified into four major types; broadband noise, rotational noise, high-speed impulsive noise, and BVI noise. ${ }^{31}$ Broadband noise is generated by a turbulent flow environment, where turbulence is present in the ambient atmosphere and the turbulent wakes of preceding rotor blades. Broadband noise usually has the lowest acoustic amplitude, out of all rotor blade noise sources. Rotational noise is a low frequency sound produced by steady and varying loads on the rotor blades, as the blades 
generate lift by rotating around the azimuth. High-speed impulsive (HIS) noise is produced by compressibility effects on the advancing blade of a helicopter when the advancing tip velocity approaches or exceeds sonic in high-speed forward flight. This type of noise is also related to the thickness of the blade since it is generated by highly localized aerodynamic events on the rotor blade, such as shock wave. Impulsive noise due to compressibility can result in a discomforting noise in high-speed forward flight. However, the noise caused by blade vortex interaction (BVI) can be more disturbing because of its impulsive nature which dominates the mid frequency noise spectrum most sensitive to human.

Although computationally more expensive than RANS methods, large-eddy simulation (LES) techniques are becoming more popular in computational aeroacoustics research nowadays, since such methods can correctly resolve a portion of the energy containing turbulent eddies which are the primary noise generating sources. The aeroacoustic investigations are based on the prediction of Sound Pressure Level (SPL). SPL is the term most often used in measuring the magnitude of sound. Sound pressure is the local pressure deviation from the ambient (average, or equilibrium) pressure caused by a sound wave. The instantaneous sound pressure is the deviation from the local ambient pressure $p_{0}$ caused by a sound wave at a given location and given instant in time. The effective sound pressure is the root mean square of the instantaneous sound pressure over a given interval of time (or space).

The Sound Pressure Level (SPL in decibels) used in the aeroacoustic analysis is given by:

$S P L=20 \log _{10}\left(\frac{p^{\prime}}{p_{0}}\right)$

where $\mathrm{p}^{\prime}$ is the instantaneous pressure and $\mathrm{p}_{0}=20 \mu \mathrm{Pa}$ is a reference sound pressure (threshold hearing). 


\section{Chapter 4: $\quad$ Numerical Simulation Results-Circular Cylinder}

\subsection{Introduction}

In the present study, the effect of a two-dimensional vortex traversing the two-dimensional flow-field past circular cylinder is investigated for $\mathrm{Re}=1.4 \times 10^{6}$, using both URANS (with a shear stress transport, SST, turbulence model) and LES (with the Smagorinsky and Lilly SGS model) approaches. The primary objective is to provide insight into the mechanisms of vortex-structure interaction, relevant to the broader practical problem of flow-induced vibrations and airfoil-vortex interactions. Two different computational approaches, URANS and LES, are used and compared. Single vortices are released separately onto a developed computation domain and the aerodynamic coefficients are calculated. The influence of horizontal miss-distances on the aerodynamics coefficients and the time required for the turbulent flow to recover after the influence of the vortex vanishes is investigated; this time will be referred to as the "recovering time". Although it is expected that the present quasi three-dimensional simulations will not capture some inherently three-dimensional turbulent structures, the practical benefits of conducting these 2-D investigation provides more detailed study of comparative effects for flows with and without imposed vortices, under a range of initial conditions. 


\subsection{Governing Equations and Numerical Method}

A finite-volume Navier-Stokes solver (CFX-11.0) was used for both URANS and LES simulations. In the following sections, the results of URANS and LES solution are presented. The numerical analysis was performed using parallel computing having 16 nodes.

\subsection{Computational Model}

In the present analysis a superimposed vortex on a uniform flow past a circular cylinder is investigated using large-eddy simulation. The 2-D simulations were performed for high Reynolds number $\operatorname{Re}=1.4 \times 10^{6}$ based on the free-stream velocity $U_{\infty}=100 \mathrm{~m} / \mathrm{s}$ and diameter of cylinder D. The flow having such a high Reynolds number exhibits a complicated flow regime with laminar separation, transition to turbulence, reattachment and another separation downstream along the cylinder surface.

Two different computational domains and different grid sizes were tested in the present analysis. A type B computational domain consisting of 2.6 million grid points with biased accumulation of grid points around the circular cylinder and a grid expansion factor of 1.01 was used for the present analysis. In the following, an overview of the computational domains, blocking structure and grid sample is presented. For all of the computations in the

present analysis, a dimensionless time step $\overline{\Delta t}=\Delta t U_{\infty} / D=7.5 \times 10^{-6}$ is chosen, where $U_{\infty}$ is the free-stream velocity and D is the diameter of cylinder. The time-step is determined with respect to the explicit time-marching scheme and temporal resolution requirement of LES 
(CFL $\leq 1)$. In the present investigations a value of Courant-Friedrichs-Lewy (CFL) number lower than 0.9 was maintain throughout the all simulations. The flow field is solved using the filtered Navier-Stokes equations (Ansys CFX 11.0) along with a standard subgrid scale model and van Driest wall damping, $l=C_{s} \bar{\Delta}\left[1-\exp \left(-y^{+} / 25\right)^{3}\right]^{0.5}$.

The core size of the superimposed vortex is equal to $\mathrm{D}$, where $\mathrm{D}$ is the diameter of the cylinder and the strength of the vortex is $25 \%$ higher than the uniform free-stream velocity $U_{\infty}$. Several different test cases were investigated based on the vortex release location $a$.

The boundary conditions were set as follows: inlet velocity $=100 \mathrm{~m} / \mathrm{s}$, zero intensity of turbulence at the inlet, opening outlet with zero pressure gage. For the top and bottom of the computational domain, free slip walls were set, while for the two sides, a symmetry type wall was chosen. No slip boundary conditions were used at the cylinder surface. In the present paper the quantities of interest are the pressure coefficient $c_{p}$, lift coefficient $c_{l}$, drag coefficient, $\mathrm{c}_{\mathrm{d}}$, and Strouhal number, $\mathrm{S}_{\mathrm{t}}$. The pressure coefficient is calculated using:

$$
c_{p}=\frac{p-p_{\infty}}{\frac{1}{2} \rho_{\infty} U_{\infty}^{2}}
$$

where $p$ is the pressure at the surface of cylinder, $U_{\infty}$ and $p_{\infty}$ is the free-stream velocity and free-stream pressure respectively.

The lift coefficient per unit span is defined as:

$$
c_{l}=\frac{L}{\frac{1}{2} \rho_{\infty} U_{\infty}^{2} l}
$$

while the drag coefficient per unit span is given by:

$$
c_{d}=\frac{D}{\frac{1}{2} \rho_{\infty} U_{\infty}^{2} l}
$$


where $L$ is the lift force, $D$ is the drag force, $\rho_{\infty}$ is the flow density and $U_{\infty}$ is the free-stream velocity and $l$ is the characteristic length (diameter of cylinder). To characterize the unsteady wake, the Strouhal number is defined as:

$$
S_{t}=\frac{f D}{U_{\infty}}
$$

where $f$ is the frequency, $D$ is the cylinder diameter and $U_{\infty}$ is the free-stream velocity.

In the present analysis two computational domains namely A and B, were used for the flow field analysis. The computational domain was generated using the ICEM-CFD mesh generator. Structured grid was used for both types of geometries A and B. For both computational domains $\mathrm{A}$ and $\mathrm{B}$ four differed grid sizes were investigated and a gird convergence analysis was performed for both geometries. The computational domains are presented in the following.

\subsubsection{Circular-cylinder computational domain type A}

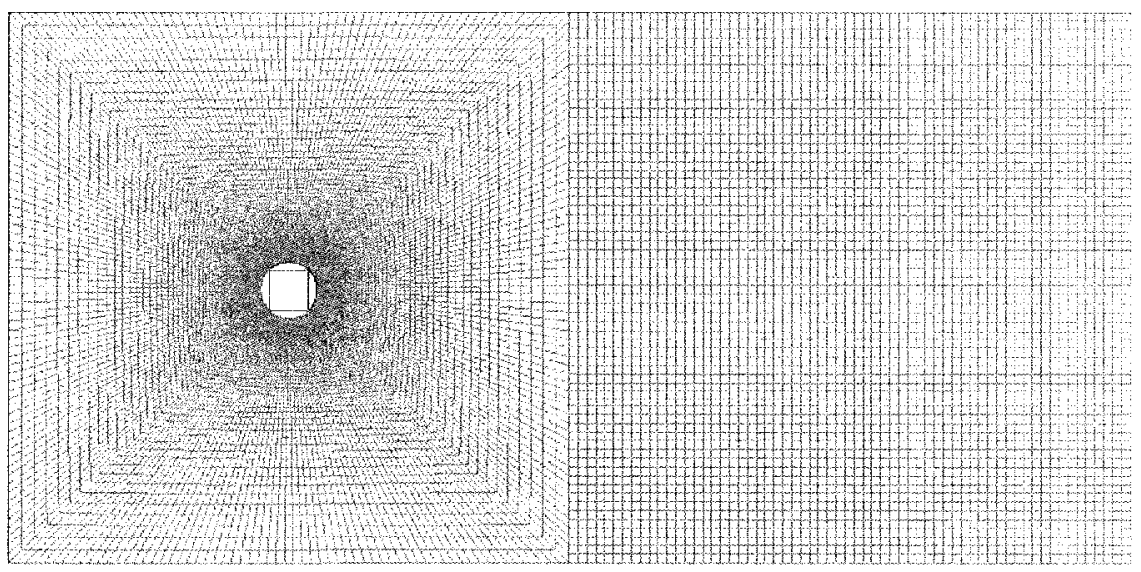

Figure 4.1 Cylindrical geometry, computational domain type A. 


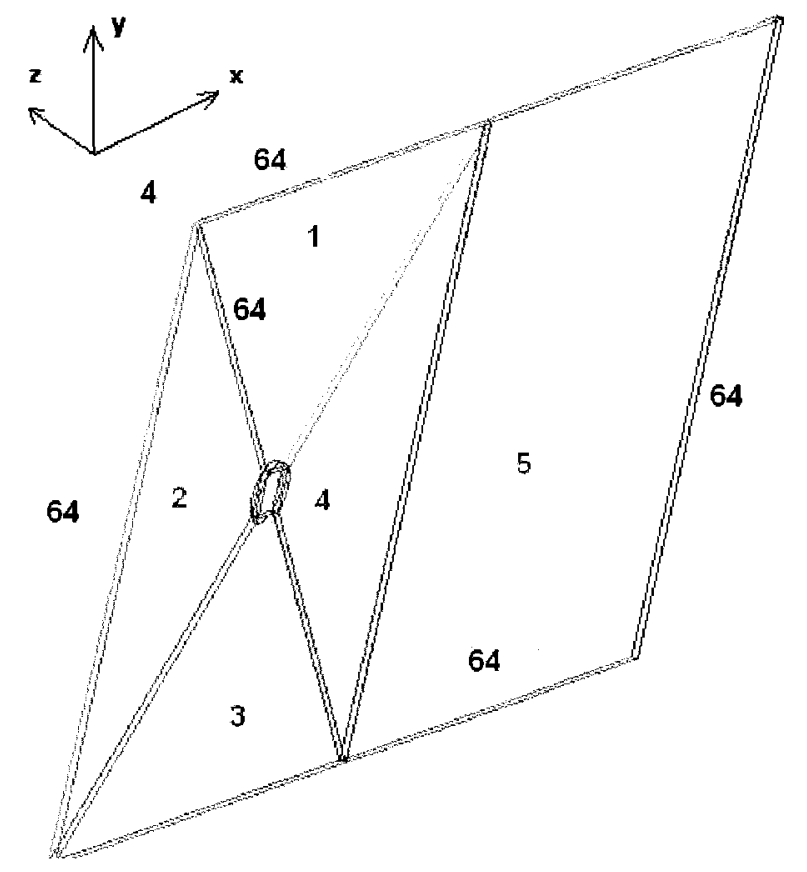

Figure 4.2 Schematic view of cylinder computational domain type A.

Figure 4.1 presents a schematic view of the computational domain with the cluster of grid points near the surface of the cylinder. As presented in the Fig. 4.2 the computational domain is divided into five different blocks. The region consisting of block 1 through 4 is O-type orthogonal grid and the region 5 is a Cartesian grid as shown in Figure 4.1.

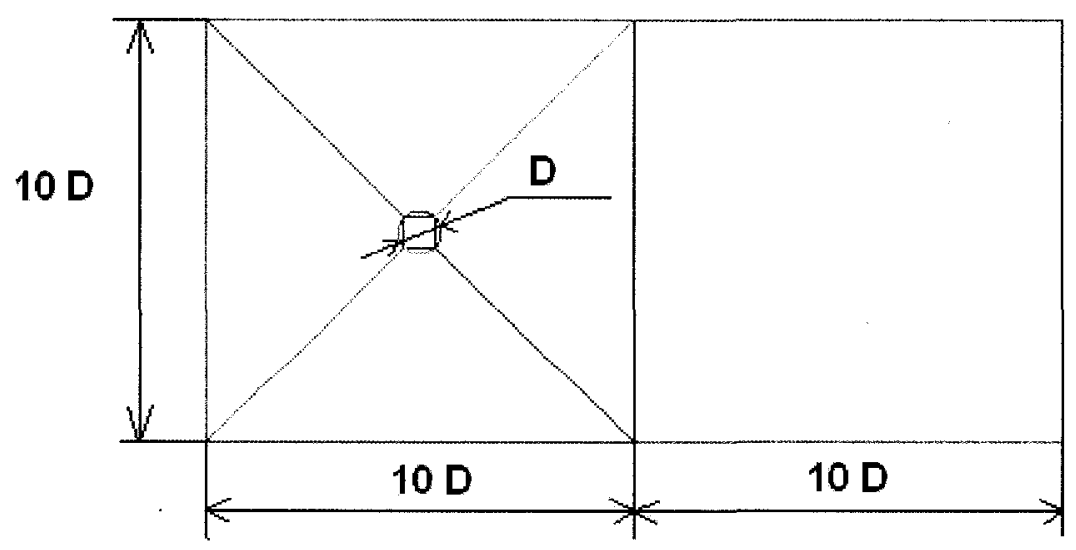

Figure 4.3 Schematic view of cylinder computational domain type A. 
The dimensions of the computational domain type A are presented in the Figure 4.3. The diameter of the cylinder $\mathrm{D}$ is $0.21 \mathrm{~m}$ and it is centered, into the O-type orthogonal grid.

The dimensions of the computational domain were based on previous numerical investigations. ${ }^{42}$ Although the present computational domain is suitable for aerodynamic investigation, a larger domain is necessary if the aeroacoustics is of concern since the wall effect is more significant.

Both computational domains have a spanwise extension of the integration domain $z_{\max }=0.1 D$. The number of nodes in the z-direction is kept constant to 4 . Four different grid sizes were analyzed as presented in the Table 4.1.

Table 4.1 Cylinder type A grid sizes.

\begin{tabular}{|c|c|c|c|}
\hline Case \# & Location of the first node from the wall & Expansion factor & Number of nodes \\
\hline 1 & 0.014 & 1.01 & 80,640 \\
\hline 2 & 0.007 & 1.01 & 325,120 \\
\hline 3 & 0.0035 & 1.01 & $1,305,600$ \\
\hline 4 & 0.002 & 1.01 & $2,153,472$ \\
\hline
\end{tabular}

For each test case there is a high density of nodes near the cylinder wall since this is the region of interest where most of the flow features are identified. The grid coarsens away from the cylinder wall in a geometric ratio manner. 


\subsubsection{Circular-cylinder computational domain type B}

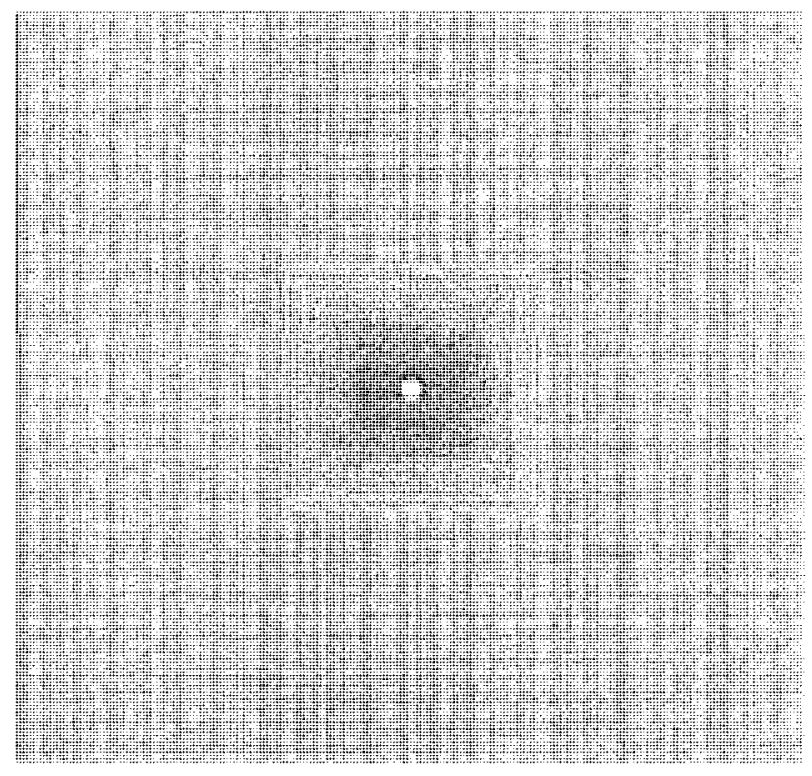

Figure 4.4 Schematic view of cylinder computational domain type B.

Computational domain type B was chosen based on previous numerical investigations for the case of uniform flow past circular cylinder. ${ }^{55}$ The computational domain type B was chosen based on the idea of eliminating the wall effects on the undisturbed flow. Therefore for this type of computational domain, the walls are located far away such that all the structures of the flow are not influenced by the walls. A schematic view of the type B computational domain is presented in the Fig. 4.4.

Figure 4.5 presents a schematic view of the multi-block computational domain. The multi-blocking approach was chosen based on computational reasons. Using parallel processing each block is computed by a single CPU, and the data at the boundary of the blocks is transferred among the CPUs. In this sense a high computational performance is achieved. The multi-block domain consists of 12 blocks with an O-type orthogonal grid 
centered in the computational domain. A schematic view of the grid nodes distribution on each side of the blocks is presented in Figure 4.5.

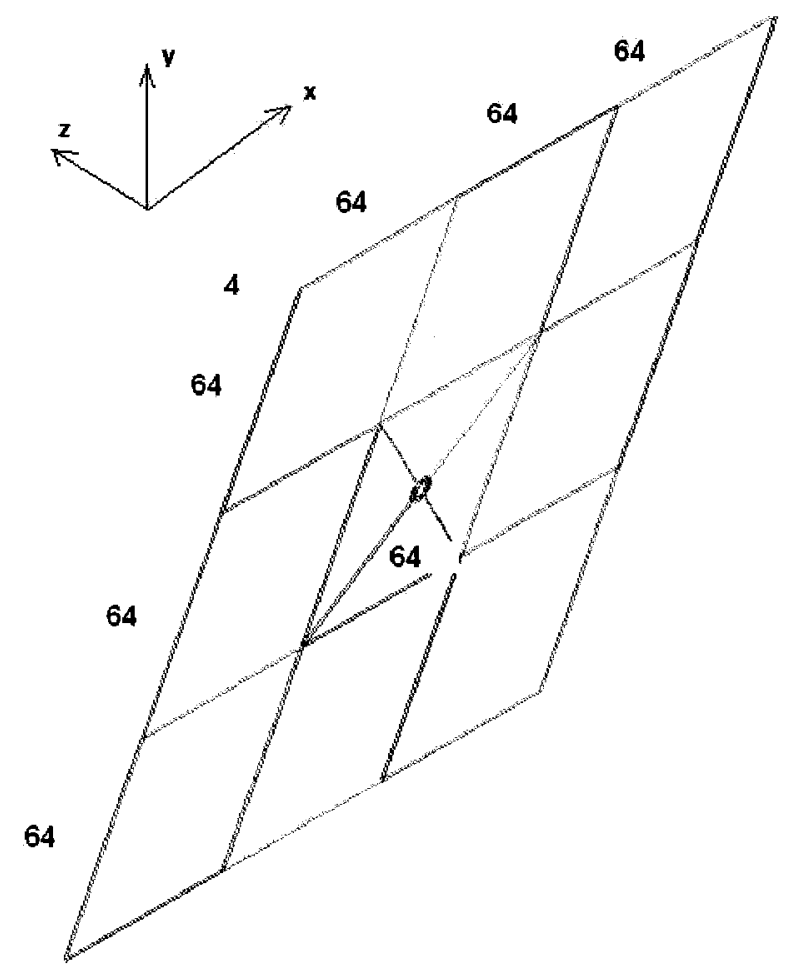

Figure 4.5 Schematic view of cylinder computational domain type B.

Similar to the computational domain type A, in the case of the computational domain type B four different grid sizes were analyzed as presented in Table 4.2.

Table 4.2 Cylinder type B grid sizes.

\begin{tabular}{|c|c|c|c|}
\hline Case \# & Location of the first node from the wall [mm] & Expansion factor & Number of nodes \\
\hline 1 & 0.0075 & 1.01 & 47,120 \\
\hline 2 & 0.0035 & 1.01 & 192,528 \\
\hline 3 & 0.0018 & 1.01 & 778,256 \\
\hline 4 & 0.0009 & 1.01 & $1,757,200$ \\
\hline
\end{tabular}


Also, the grid density is higher near the wall region and coarsens in the outward direction, in a geometric ratio with the expansion factor 1.01, as presented in Table 4.2.

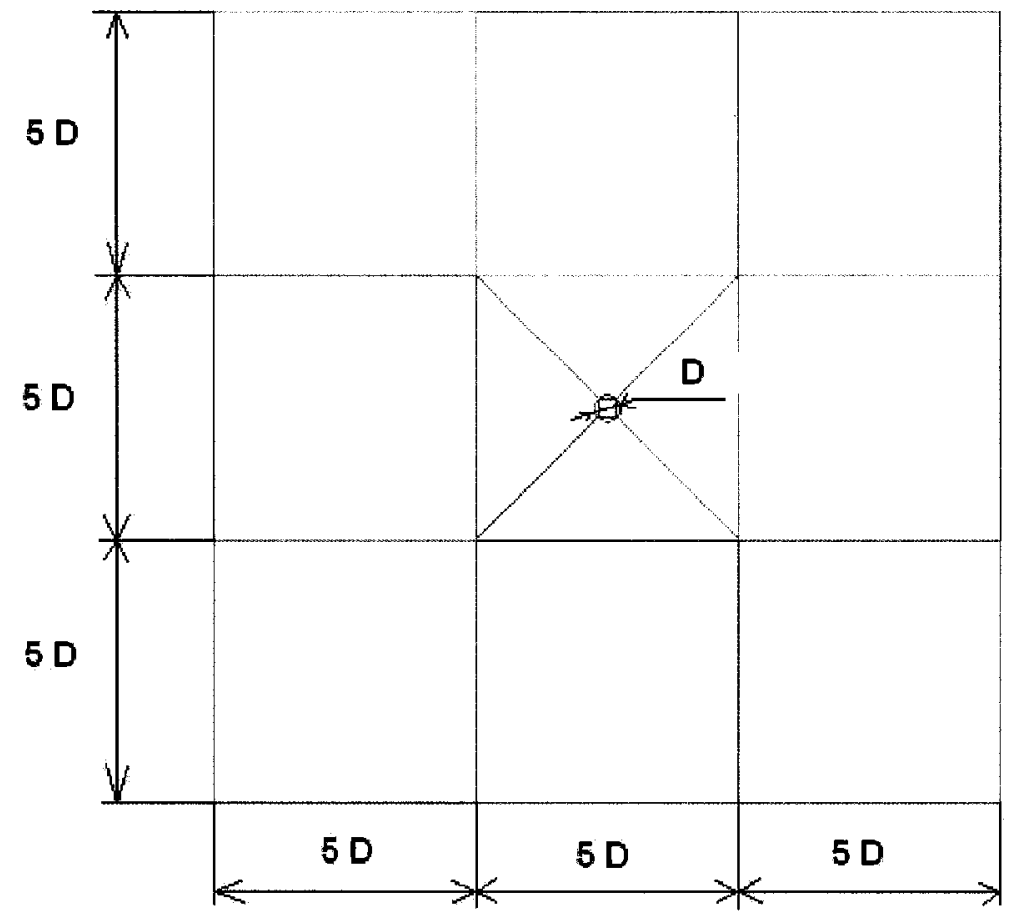

Figure 4.6 Schematic view of cylinder computational domain type B.

Figure 4.6 presents a schematic view of the multi-block type B computational domain for circular cylinder. The choice of such large computational domain ensures that the wall effect is avoided. The dimensions of the computational domain are based on the cylinder diameter. For both types of computational domains $\mathrm{A}$ and $\mathrm{B}$, a grid convergence analysis is performed to define the optimum grid size. A grid convergence analysis is presented in the following sub-section. 


\subsubsection{Grid Convergence Analysis}

As already mentioned in the previous section, a grid convergence analysis was performed for both types of computational domains $\mathrm{A}$ and $\mathrm{B}$, and four different grid sizes were considered for each type of computational domain. From the grid convergence analysis it can be seen that for type A computational domain a number of about 80,000 nodes is sufficient for accurate RANS results. The reason of choosing a denser grid was based on LES requirements, since a finer grid would perform better in terms of results accuracy. In the case of type B grid a number of about 190,000 nodes are sufficient for a good RANS solution accuracy. The grid convergence analysis for both types of grid A and B is presented in the following.

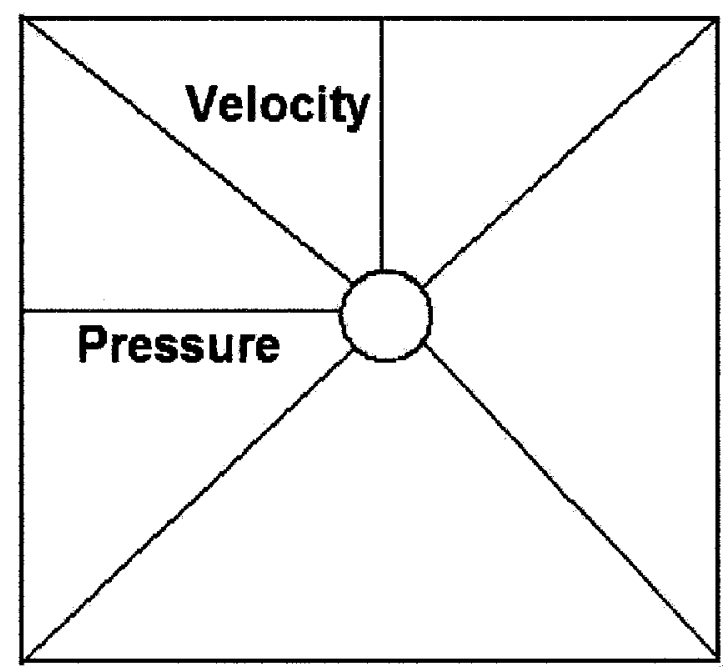

Figure 4.7 Schematic view of interrogation lines used for grid convergence analysis. 
The velocity based grid convergence has been performed based on the RANS simulations results. In this sense the averaged velocity and pressure along a vertical respectively horizontal line (in the computational domain) as presented in Figure 4.7, is recorded for each grid size and compared against each other. The choice for the present interrogation lines is based on the fact that largest changes in velocity and pressure field occur in a vertical respectively horizontal direction. Hence is expected that these two directions to provide enough information for a qualitatively comparison.

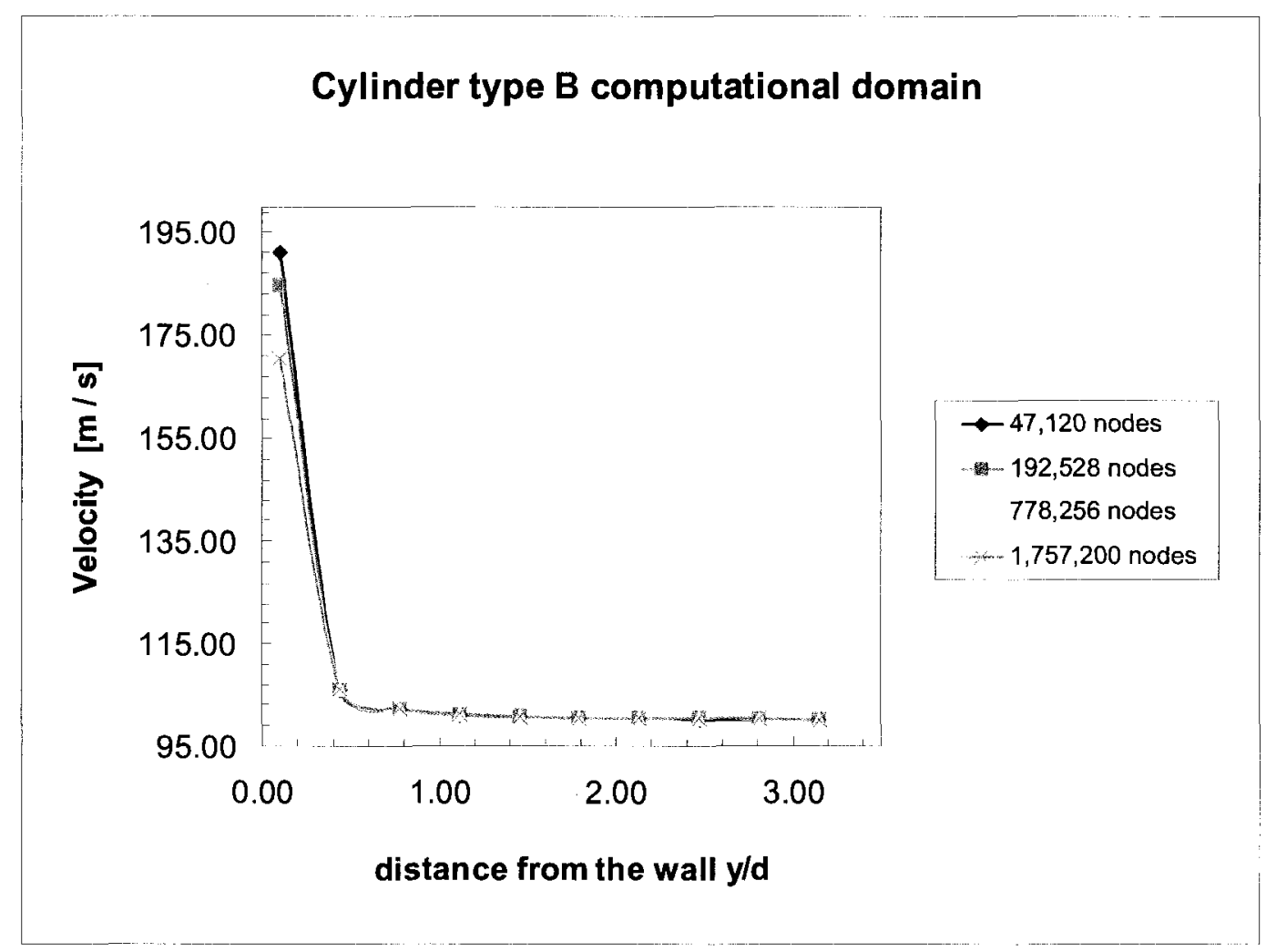

Figure 4.8 Grid convergence analysis. 


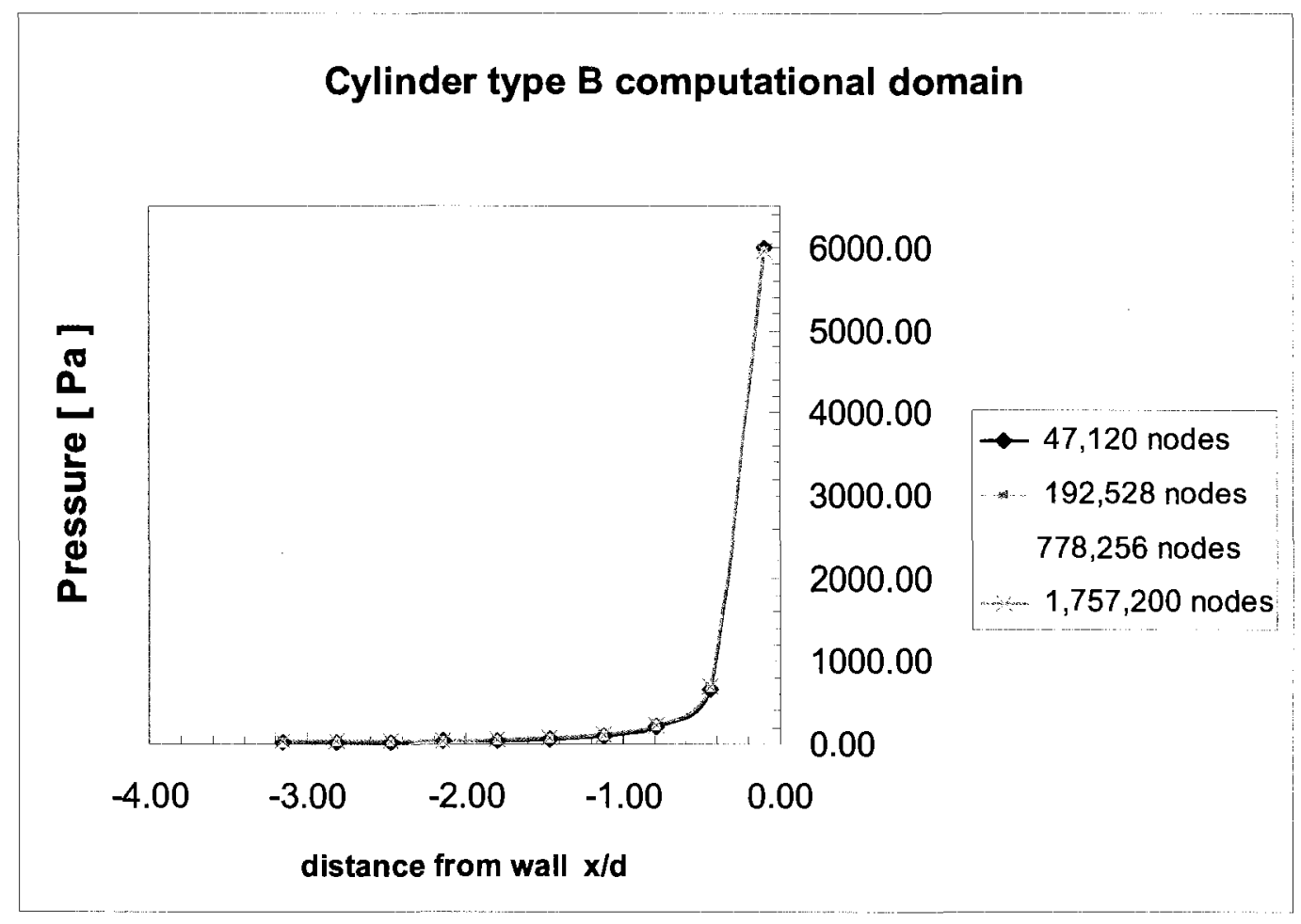

Figure 4.9 Grid convergence analysis.

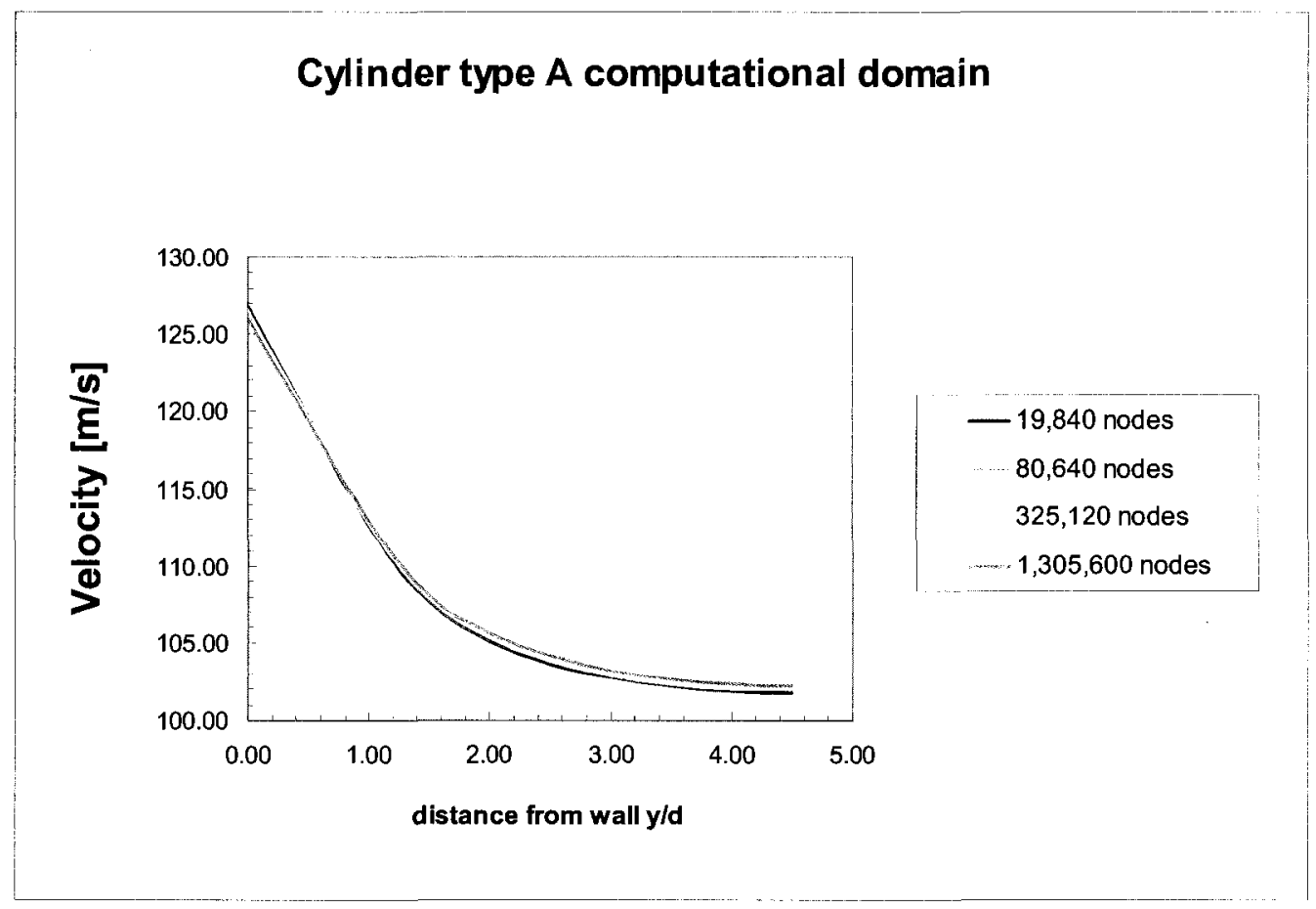

Figure 4.10 Grid convergence analysis. 


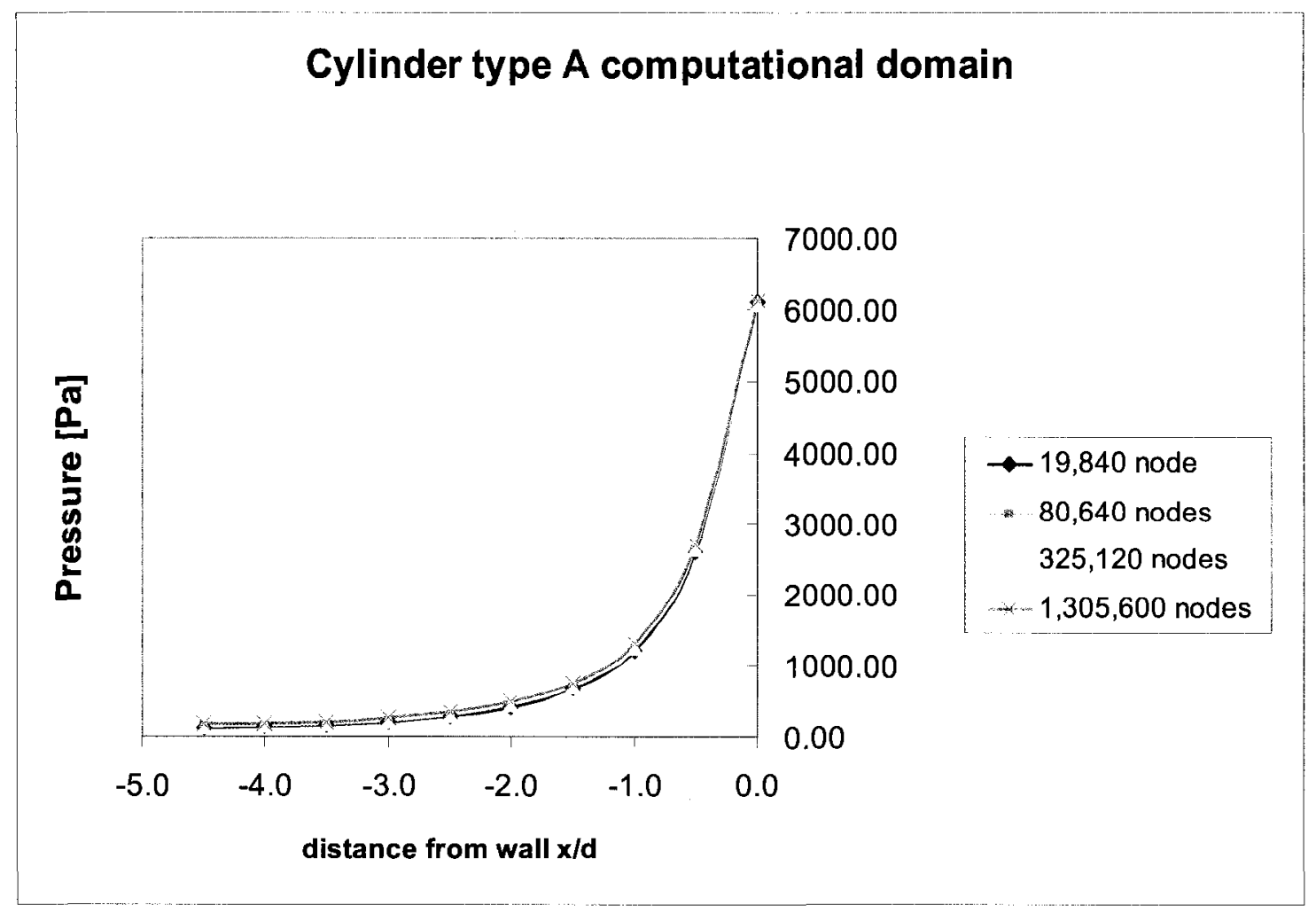

Figure 4.11 Grid convergence analysis.

Figures 4.8 and 4.9 presents the grid convergence analysis for type A computational domain, while Figures 4.10 and 4.11 presents the grid convergence analysis using a type B computational domain. It is important to be mentioned that although two different computational domains were investigated, the entire CFD analysis is based on the type B computational domain. It is expected that the computational domain type B would provide a more accurate solution since it eliminates the wall effect. 


\subsection{Aerodynamic Results}

\subsubsection{Uniform flow past circular cylinder}

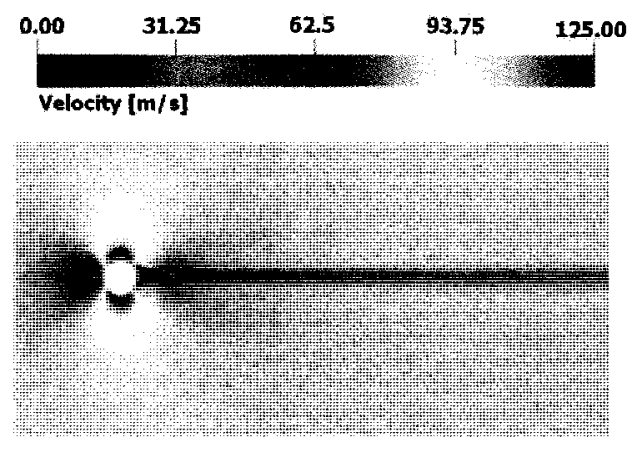

a. Velocity field

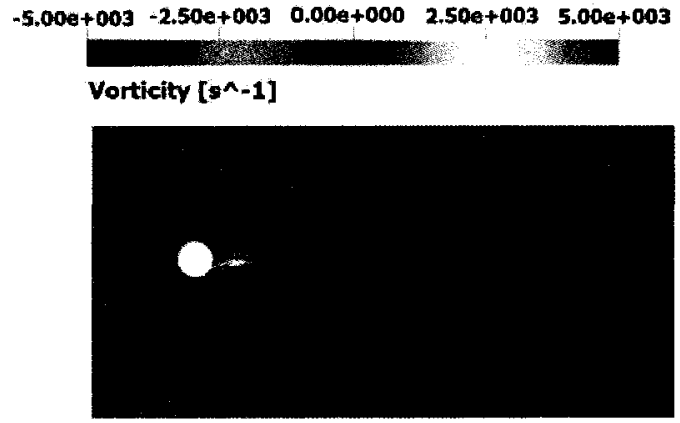

b. Vorticity field

Figure 4.12 Flow field solution (RANS).

Figure 4.12 presents the velocity and respectively vorticity flow field solution using RANS along with an SST turbulence model, using the type B computational domain. As expected, from Figure 4.12 it can be seen that RANS cannot capture the instantaneous flow field fluctuations and the von Kármán vortex street. The symmetry in the flow field (upper and lower surface of the cylinder) implies zero lift and the absence of oscillations results in a constant drag force.

The non-dimensional distance $\left(\mathrm{y}^{+}\right)$was calculated based on the formula:

$y^{+}=\frac{u^{*} y}{v}$

where $u^{*}$ represents the friction velocity at the nearest wall, $y$ is the distance to the nearest wall and $v$ is the local kinematic viscosity of the fluid. 


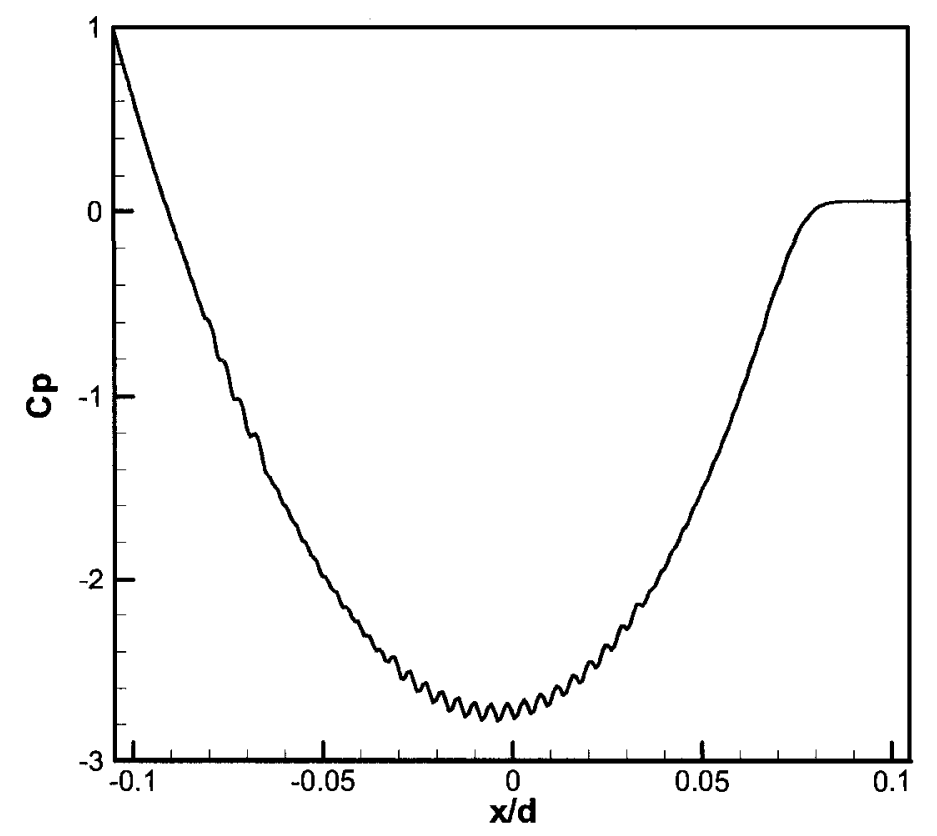

Figure 4.13 Pressure coefficient from RANS.

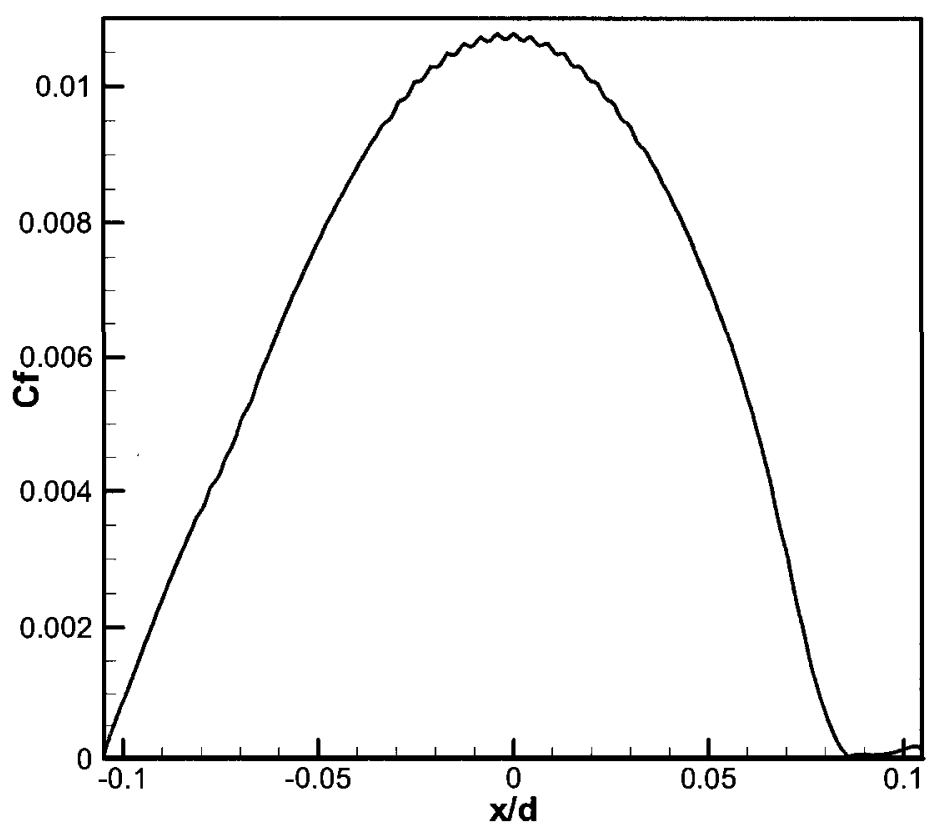

Figure 4.14 Skin friction coefficient from RANS. 
The $\mathrm{y}^{+}$value for RANS simulations was determined as being 18 , so smaller than 20 (see ANSYS-11.0).

The pressure coefficient distribution at the cylinder surface is shown in Figure 4.13. The pressure is maximum at the stagnation point and gradually decreases along with the front half of the cylinder followed by a gradually increase towards the rear. The pressure has a minimum at the top and bottom of the cylinder where the velocity presents a maximum. The velocity has a minimum at the stagnation point. From pressure coefficient distribution along the cylinder surface, it can be observed that the values of pressure coefficient along the upper and lower surface of the cylinder overlap. This result implies that the lift coefficient is zero since the lift coefficient represents the surface integral of pressure, or in other words the enclosed area between the upper and lower surface pressure coefficient curves. Since the enclosed area between the two curves is zero; it means no lift is generated. Figure 4.14 shows the skin friction coefficient distribution along the cylinder surface indicating positive values. The $\mathrm{y}^{+}$value for LES simulations was determined as being equal to 1 , and 15 grid points were used across the boundary layers (see ANSYS-11.0). 


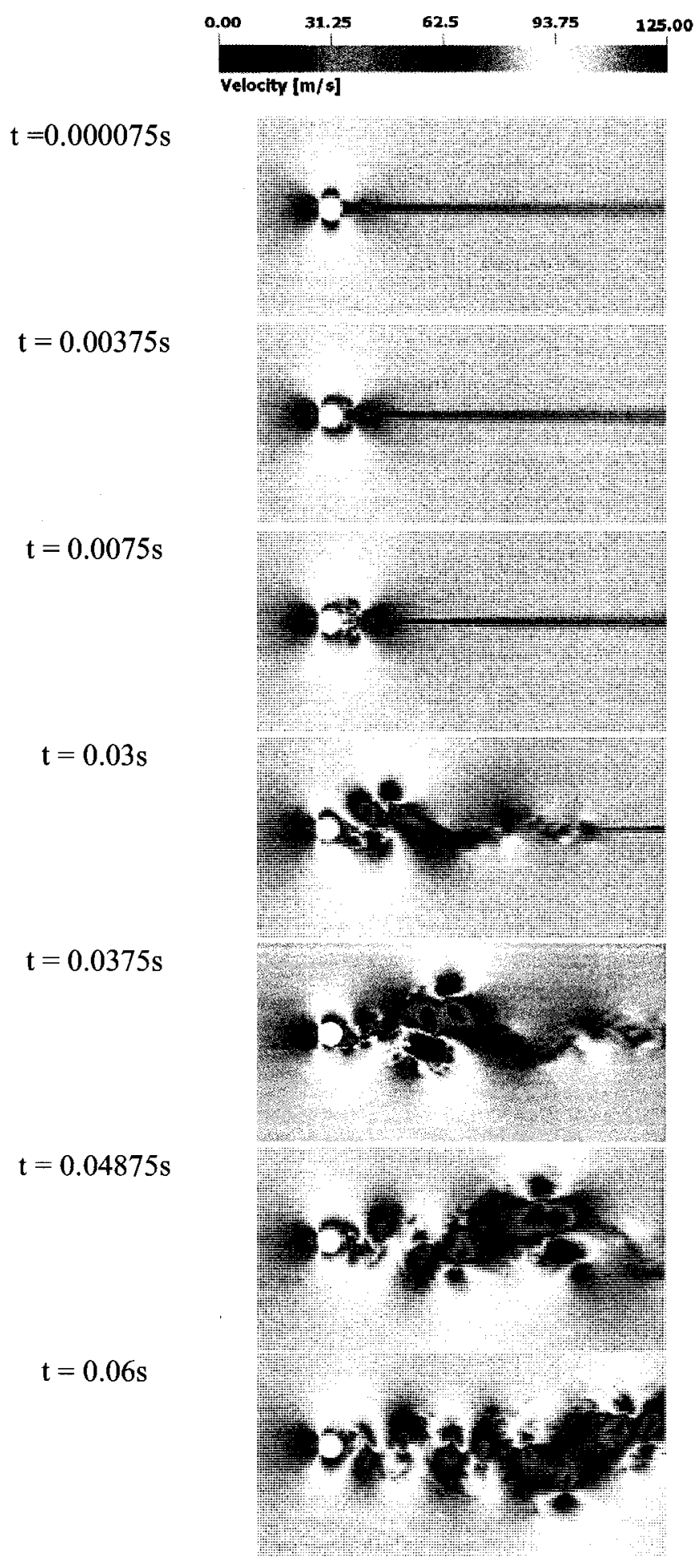

a. Velocity field
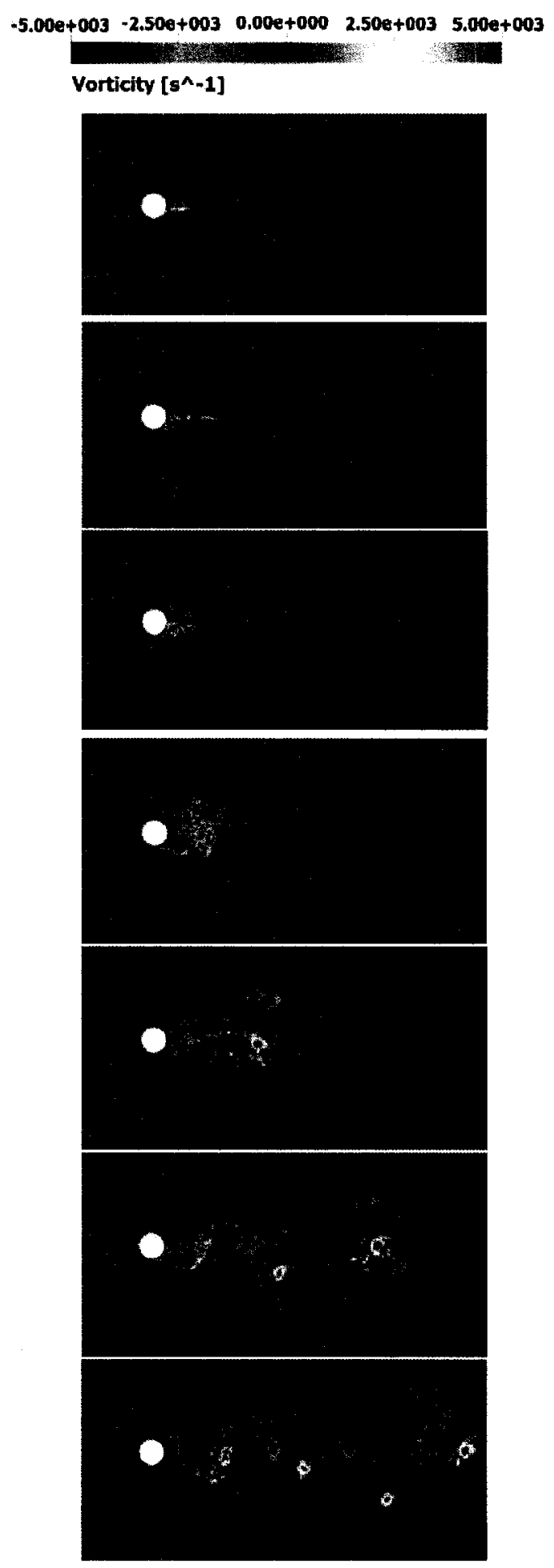

b. Vorticity field

Figure 4.15 Time developing fluid structures of a uniform flow past circular cylinder (LES). 
Figure 4.15 shows the time developing flow structures, using large-eddy simulation. The flow field is initialized from a converging RANS solution using the SST turbulence model. The velocity and vorticity field solutions at different instants in time are shown in the figure. The large-eddy simulation accurately captures the physics of the uniform flow past a circular cylinder and reproduces all the important fluid structures including the boundary layer separation, the formation of vortices, and formation of a highly turbulent wake region behind the cylinder. The pressure inside the wake region remains low as the flow separates and a net pressure force (pressure drag) is generated.

The boundary layer separates from the surface and forms a free shear layer which is highly unstable. As the flow develops, this shear layer eventually rolls into a discrete vortex before detaching and shedding from the surface. The simulation also captures the interaction of the shear layer vortices shed alternately from both top and bottom surfaces, forming the familiar von Kármán vortex street.

From Figure $4.15 \mathrm{a}$, it can be seen that as the shed vortices travel downstream, they tend to distance from the mean line (passing through the center of cylinder). It also can be seen that as the separation occurs at the rear of the cylinder, the stagnation point starts to oscillate moving along the upper and lower cylinder surface. The oscillation frequency of the stagnation point is the same as the frequency of the von Kármán vortices. 


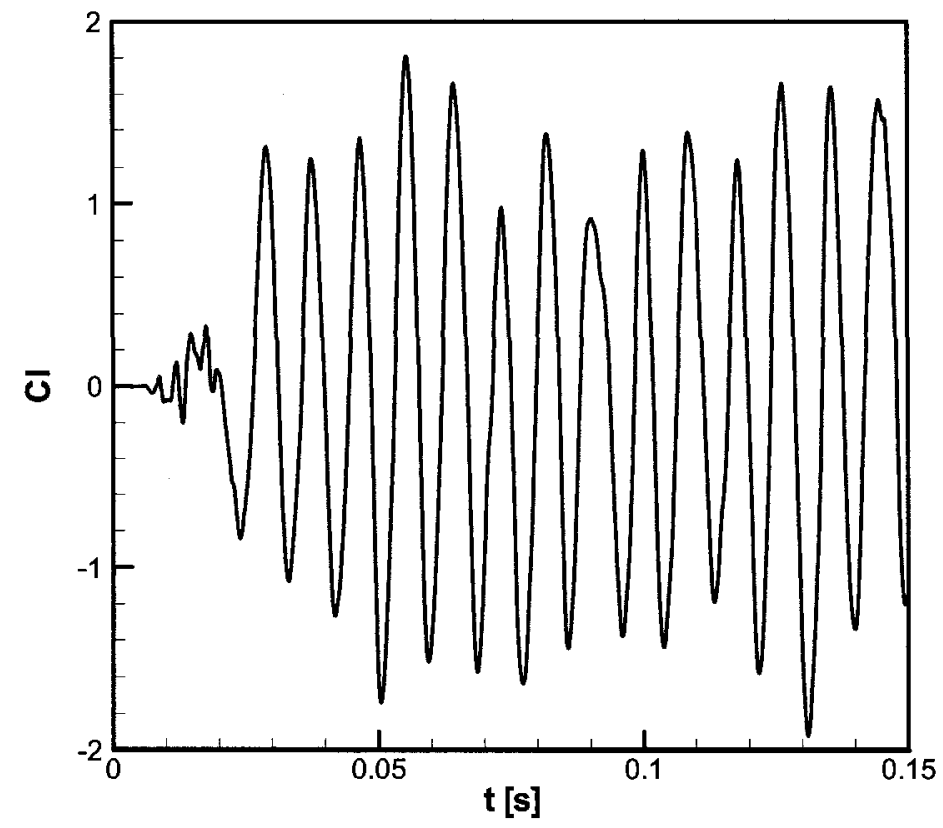

Figure 4.16 Uniform flow past circular cylinder, time history of lift coefficient.

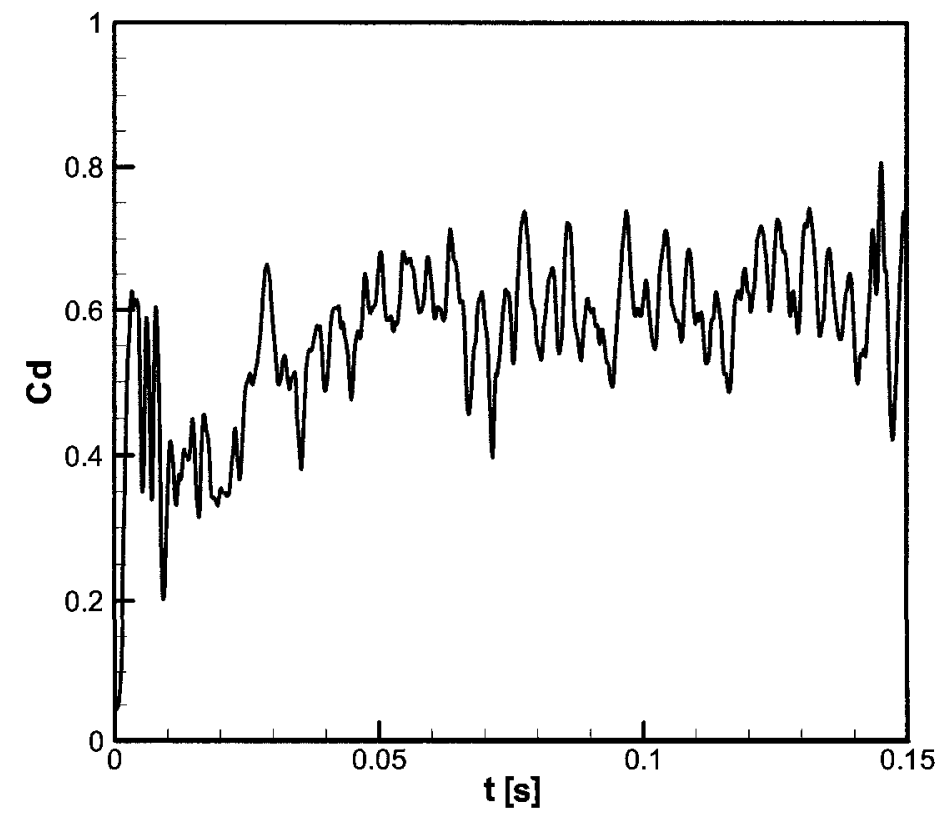

Figure 4.17 Uniform flow past circular cylinder, time history of drag coefficient. 
The oscillations of the von Kármán vortex street are reflected into the oscillations of the lift coefficient, as shown in Figure 4.16. Figure 4.16 presents the time-varying lift coefficient for a number of 20,000 time steps. It can be seen that as long as the flow remains attached to the surface, the lift coefficient is zero. As the boundary layer separates from the surface and the flow starts to separate at the rear of the cylinder, a region of high pressure and low velocity are formed, generating a vertical lift. Figure 4.16 also shows that at the beginning of the boundary layer separation process, the amplitudes of lift coefficient fluctuations are small, but these increase as the separation becomes more dominant at the rear of the cylinder. The amplitudes of lift coefficient fluctuations are not uniform since the vortices traveling downstream continue to interact and merge, further perturbing the flow field and adding additional instability into the pattern of oscillations. Since these oscillations alter the net pressure force on the cylinder, the drag force is also affected as shown in Figure 4.17.

From the present computations the average of maximum peaks of lift coefficient was found to be $C_{l}=1.3$ and the average of maximum peaks of drag coefficient was found $C_{d}=$ 0.625. Although the results from the present two-dimensional computation are slightly higher than the experimental values ${ }^{34}, C_{d}=0.532$, they are in good agreement with the twodimensional numerical results obtained by for $\operatorname{Re}=10^{6} .{ }^{57}$ A value of drag coefficient $C_{d}=$ 0.607 was reported. The slightly differences between the results of the two computations are attributed to the Reynolds number differences.

In previous separate studies, an over prediction of the two-dimensional LES simulation results compared to the three-dimensional simulation for $\mathrm{Re}=300$ and $\mathrm{Re}=1000$ for flow past a cylinder was observed. ${ }^{43}$ Another previous analysis showed that the difference between the results from two-dimensional calculations and three-dimensional flow 
increases with the Reynolds number. ${ }^{50}$ The higher values of the drag coefficient for the twodimensional simulations were attributed to higher level of Reynolds stresses, resulting in a shorter formation length behind the bluff body. In general, the two-dimensional computations over predict the drag coefficient. However, at the critical Re and beyond, the twodimensional computations result in a fairly good prediction of the time-averaged drag coefficient.

From the present investigation the Strouhal number based on lift coefficient variation was found $S_{t}=0.236$, slightly higher than 0.22 value obtained from experiments. ${ }^{34}$ Although over predicted, the Strouhal number value from the present simulation is in good agreement with the value $\mathrm{S}_{\mathrm{t}}=0.27$ for $\mathrm{Re}=3.6 \times 10^{6}$ reported in previous investigations. ${ }^{57}$

\subsubsection{Superimposed vortex on a uniform flow past circular cylinder}

Single vortices were released separately onto a fully developed LES flow field (after 20,000 time steps). Figure $4.18 \mathrm{a}$ and $4.18 \mathrm{~b}$ shows simulation results of the velocity magnitude for a counter-clockwise rotating vortex traveling with the uniform flow and interacting with the cylinder, using LES and URANS, respectively. The vortex is released at the horizontal location of $\mathrm{a}=-0.42 \mathrm{~m}$. For both simulations URANS and LES, the same grid and time step were used. 


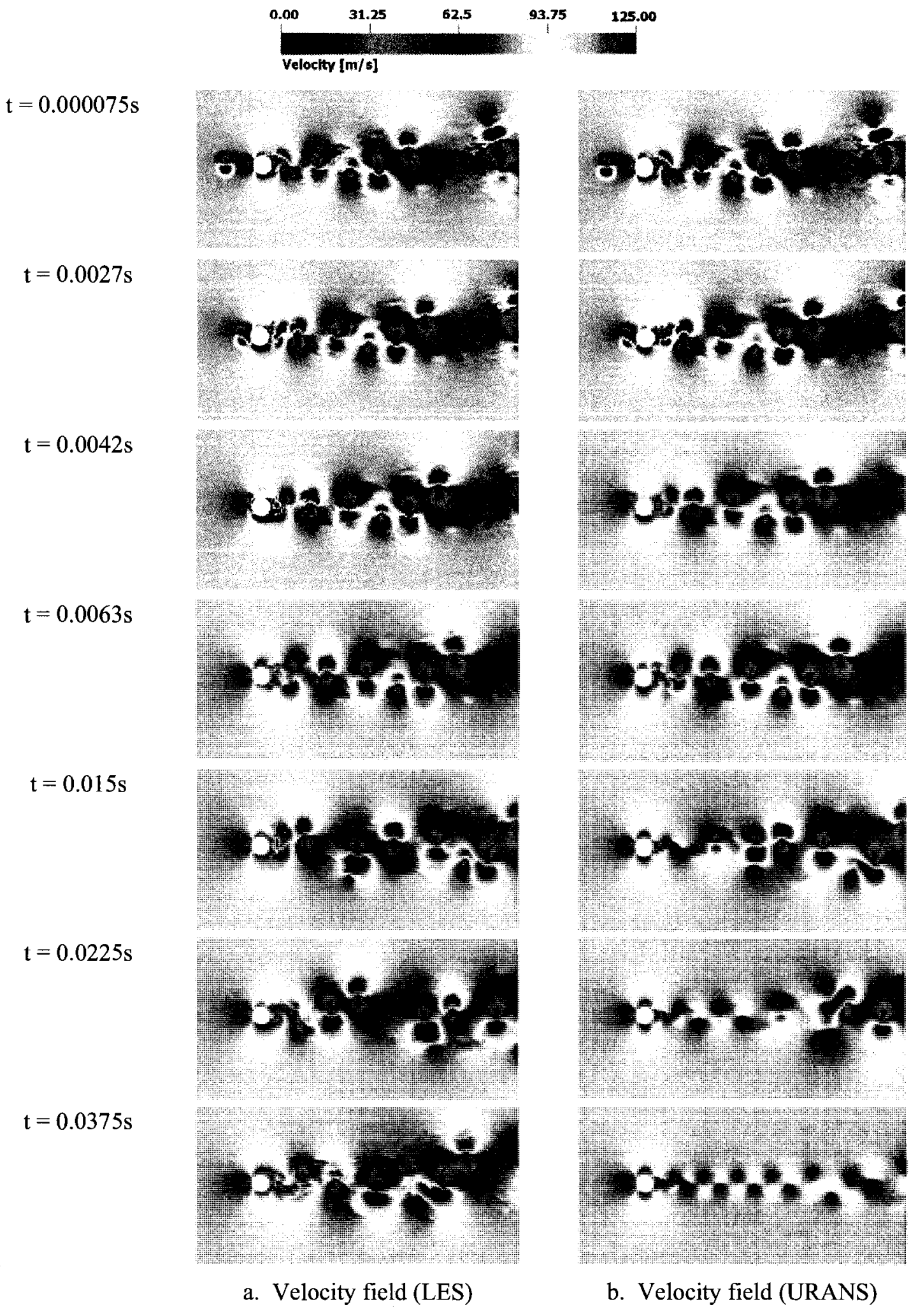

Figure 4.18 Time developing fluid flow structures of a superimposed vortex on uniform flow past cylinder. 


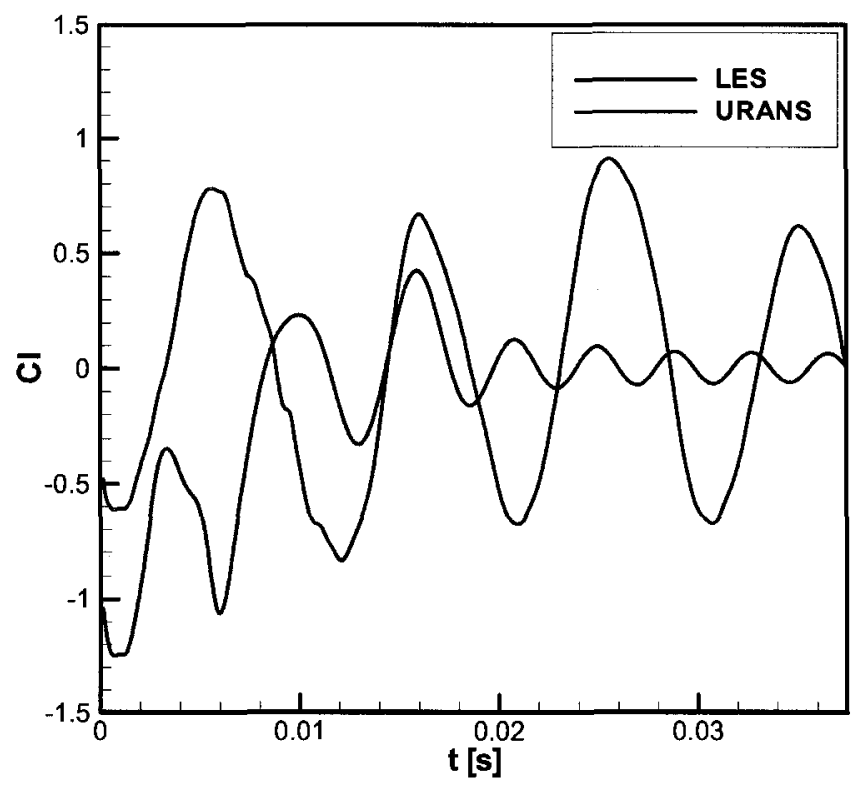

Figure 4.19 Superimposed vortex on uniform flow past a cylinder, time history of lift coefficient.

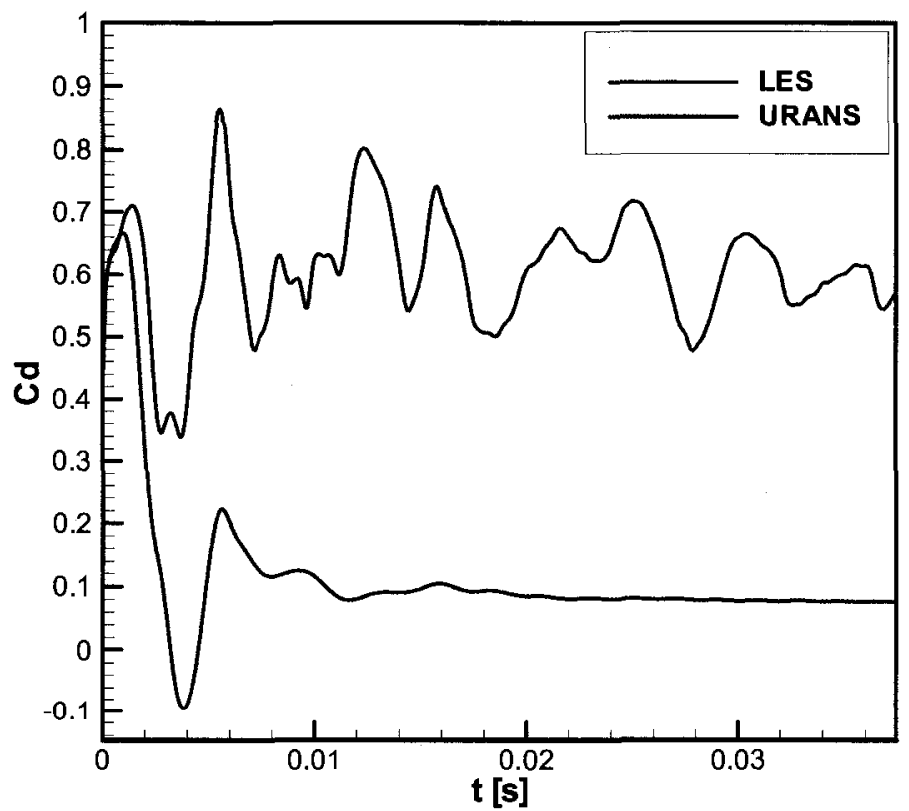

Figure 4.20 Superimposed vortex on uniform flow past a cylinder, time history of drag coefficient. 
Figures 4.19 and 4.20 present the comparison of aerodynamic coefficients using LES and URANS flow field solution. The lift coefficient from URANS solution oscillates with smaller amplitude and shorter period when compared against LES, as seen in Figure 4.19. The drag coefficient from URANS solution converges asymptotically to a value around $\mathrm{C}_{\mathrm{d}}=$ 0.07 as the residual component of the vortex merges with von Kármán vortex street. The drag coefficient from LES solution oscillates around $C_{d}=0.58$, after the vortex-cylinder interaction. From the present comparison it is observed that using the LES approach, the characteristics of the flow structures are well preserved, while URANS is quite dissipative.

The dissipative nature of URANS has been identified by previous studies. ${ }^{55-56}$ The turbulence model was identified as the main cause of the strong dissipation. 


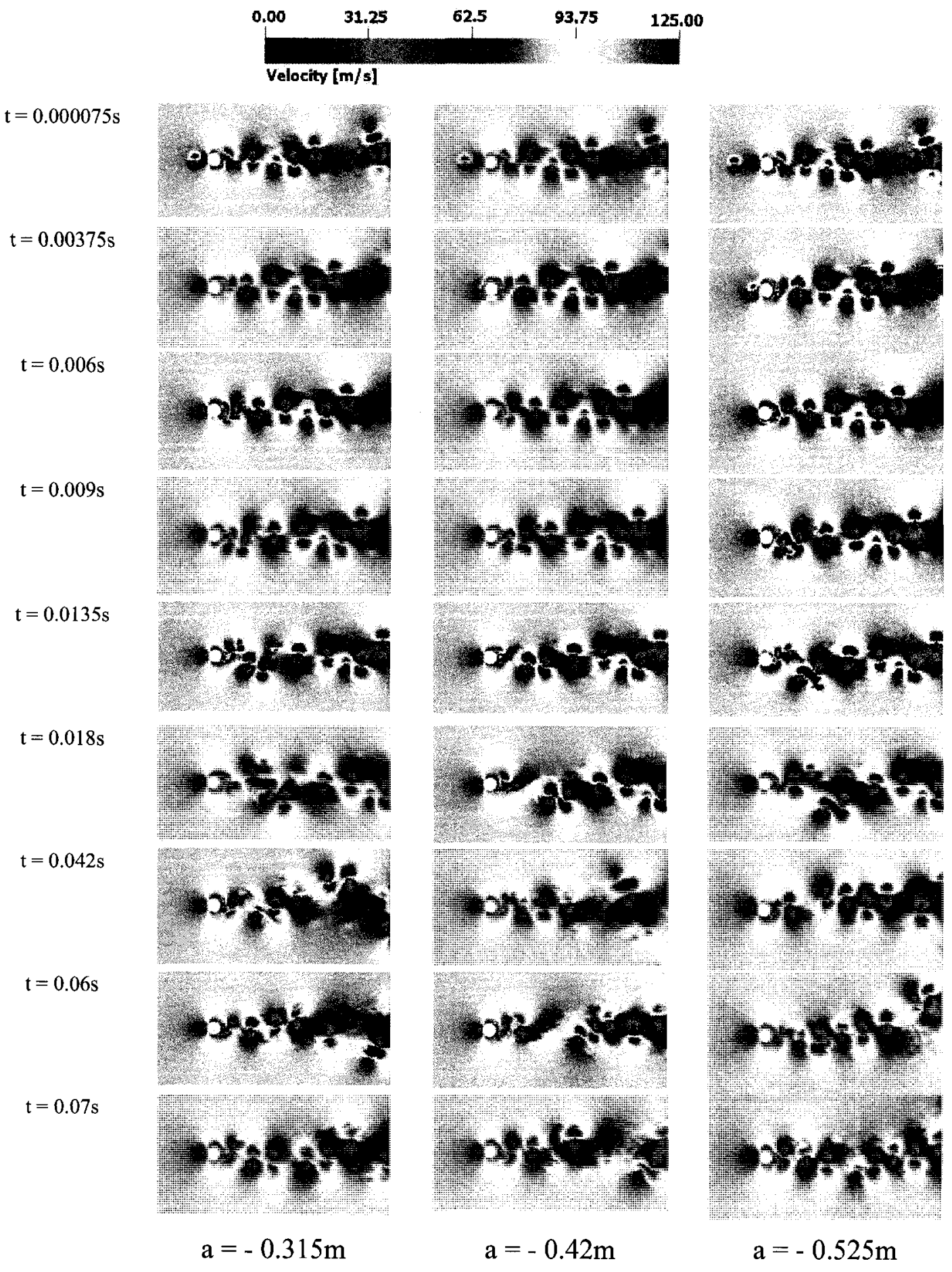

Figure 4.21 Time developing fluid structures of a superimposed vortex on a uniform flow past circular cylinder. 


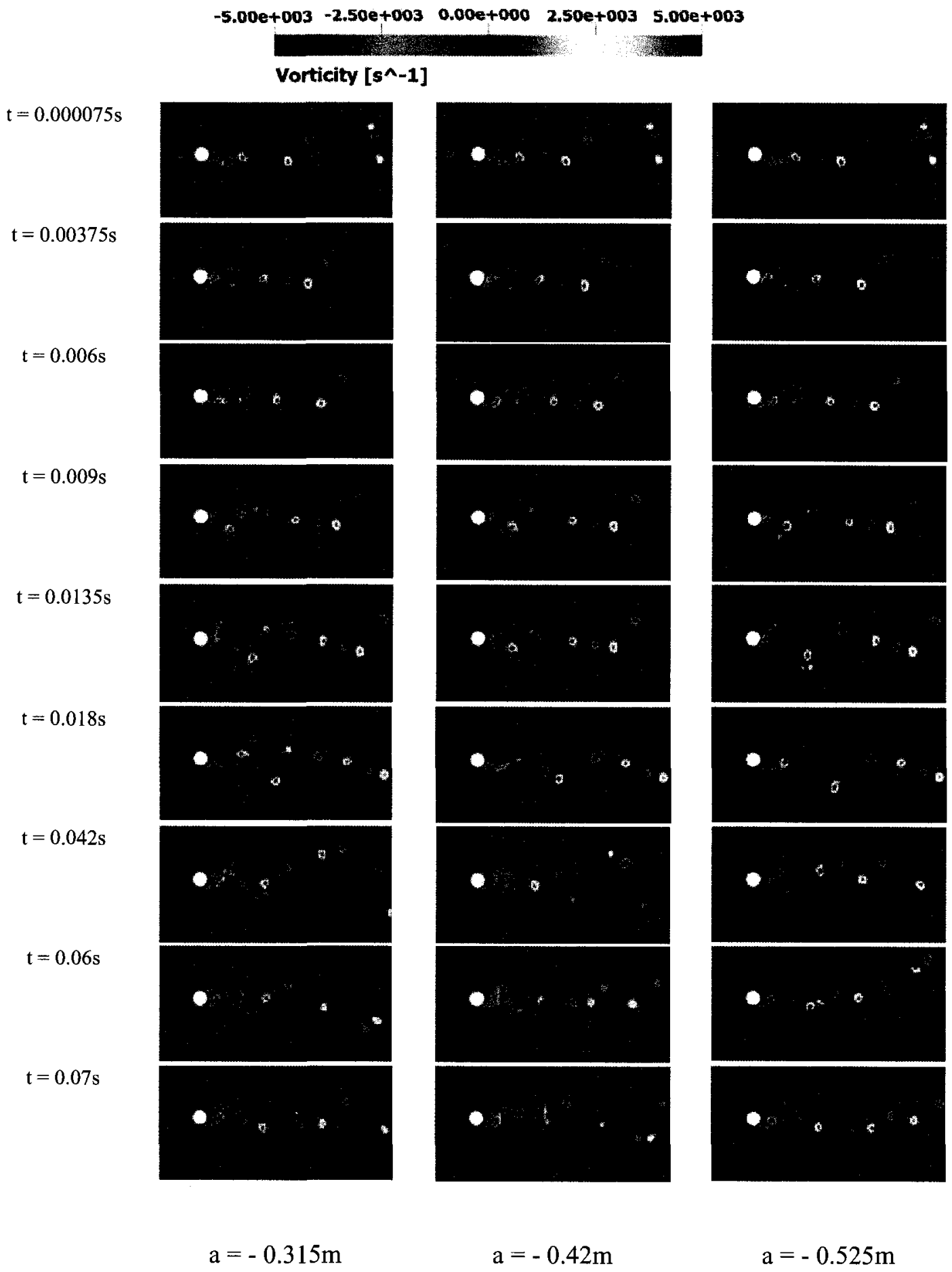

Figure 4.22 Time developing fluid structures of a superimposed vortex on a uniform flow past circular cylinder.

Figure 4.21 presents the LES results of velocity magnitude when a clockwise rotating vortex is released at three different locations upstream cylinder, $\mathrm{a}=1.5 \mathrm{D}, \mathrm{a}=2 \mathrm{D}$ and $\mathrm{a}=2.5 \mathrm{D}$. It 
can be seen that in all test cases, as the clockwise rotating vortex approaches the cylinder, the cylinder sees an induced velocity from the vortex pointed downward. This induced velocity has an impact on the pressure field at the cylinder surface and implicitly on the lift and drag coefficient. When the vortex approaches the cylinder, the stagnation point moves upwards until the vortex encounters the cylinder and the vortex core is distorted.

From Figure 4.21, it is apparent that the vortex core is destroyed as it interacts with the cylinder and there are perturbations in the von Kármán vortex street due to the presence of the vortex in the flow field. Also it can be seen that as the vortex approaches the cylinder the velocity distribution on the upper and lower surface of the cylinder varies continuously until the vortex merge into the von Kármán vortex street.

The lift coefficient value reaches a peak at the instant when the vortex core is centered with the centre of the cylinder, at about $\mathrm{t}=0.0042 \mathrm{~s}$. As the vortex distorts and merges into the von Kármán vortex street, the lift coefficient decreases and follows a periodically oscillating pattern as seen in Figure 4.23.

It can be seen that the amplitudes of the lift coefficient are larger when the superimposed vortex is present into the flow field, compared with the values for the case of uniform flow past cylinder. This is a result of the fact that the presence of the vortex alters the flow field by inducing a high velocity at the surface of the circular cylinder which consequently affects the pressure field.

From the vorticity field in Figure 4.22 , it can be seen that the vortices are somehow aligned along two main paths of negative slopes as it can be clearly seen at the instant $t=$ $0.018 \mathrm{~s}$. At instants of time $\mathrm{t} \geq 0.042 \mathrm{~s}$ the vortices do not follow any pattern. The influence of the vortex-cylinder mechanism of interaction on the flow field can be seen as well from the 
vorticity field comparison, where for the test cases $\mathrm{a}=-1.5 \mathrm{D}$ and $\mathrm{a}=-2 \mathrm{D}$ the vortices fall into a narrowed band while for the test case $\mathrm{a}=-2.5 \mathrm{D}$ the vortices fall into a larger band. Also the vortices present higher magnitude for test case $\mathrm{a}=-1.5 \mathrm{D}$ and $\mathrm{a}=-2.5 \mathrm{D}$ than for test case $\mathbf{a}=-2 \mathrm{D}$, and this is associated with the vortex-cylinder mechanism of interaction as well.

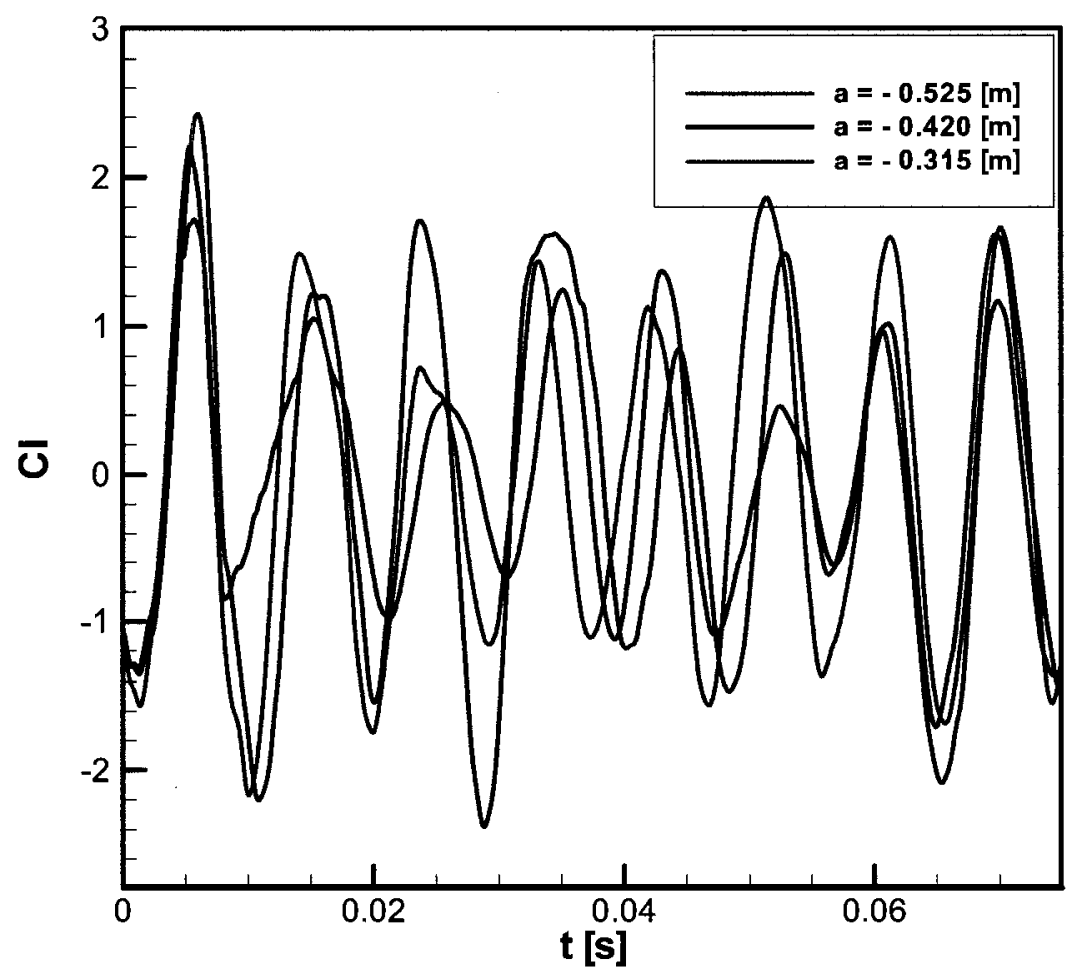

Figure 4.23 Superimposed clockwise rotating vortex on uniform flow past cylinder, lift coefficient comparison.

Plots of the time-varying lift coefficient show that the instant of vortex-cylinder interaction has significant influence on the vortex-cylinder mechanism of interaction. A decrease of lift coefficient peaks corresponding to the decrease of vortex-cylinder horizontal miss-distance can be seen at the instant when the vortex and cylinder are centered, about the instant $t=$ 0.042s. Although shifted in time, the lift coefficient values follow a similar pattern for all test 
cases as seen in Figure 4.23. At about the instant $\mathrm{t}=0.06 \mathrm{~s}$, the lift coefficient values almost perfectly overlap, suggesting that independent of the vortex releasing location, the influence of the vortex on the flow field is wiped out at about the same time and hence the "recovering time" is about the same. This is due to the fact that for all test cases, the vortex encounters the cylinder frontally such that the interaction is so severe that the vortex loses its characteristics and hence it has less impact on the flow field as the time elapses. In the present analysis, the lift coefficient is used in order to estimate the Strouhal number although any other flow variable such as, velocity or vorticity could also be used for the wake description. From the present analysis it was found that the presence of the vortex in the flow field either increases or decreases the value of Strouhal number, depending on the vortex releasing location (or instant of interaction). This means that the frequency at which the vortices are shed is higher or lower than in the case of a uniform flow past circular cylinder, depending on the specific vortex-cylinder mechanism of interaction. Consequently for the case of a clockwise rotating vortex interacting with a cylinder, the Strouhal number decreases with the decrease of the vortex-cylinder horizontal miss-distance. The values of Strouhal number corresponding to the vortex-cylinder miss-distances $a=-0.315 \mathrm{~m}, \mathrm{a}=-0.42 \mathrm{~m}$ and $\mathrm{a}$ $=-0.525 \mathrm{~m}$ where found as being is $S_{t}=0.2275, S_{t}=0.2345$ respectively $S_{t}=0.245$. The values of Strouhal number are based on the lift coefficient frequency, using the last $0.03 \mathrm{~s}$, in order to eliminate the initialization effects. 


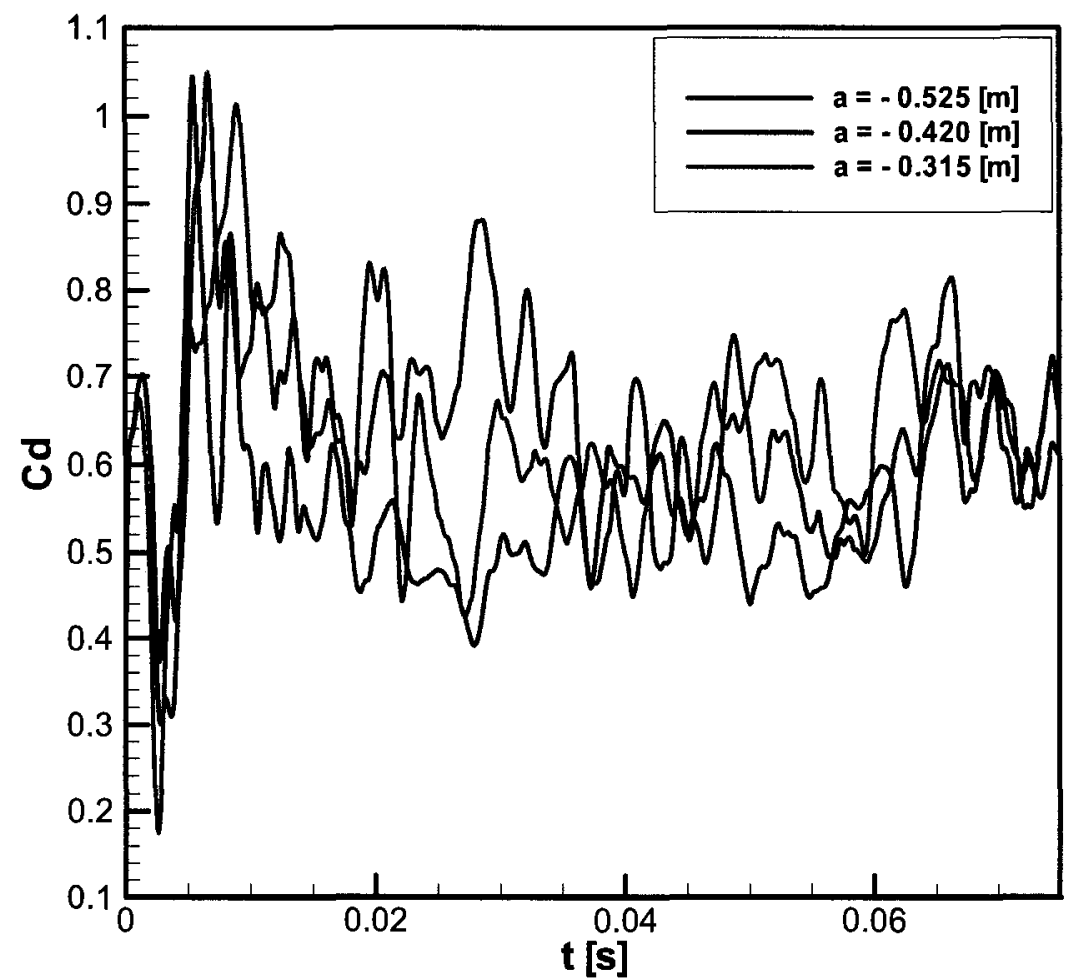

Figure 4.24 Superimposed clockwise rotating vortex on uniform flow past cylinder, drag coefficient comparison.

Figure 4.24, shows the comparison of drag coefficient for the three test cases as a function of vortex-cylinder horizontal miss-distance. Although slightly shifted in time, the drag coefficient values follow a similar trend presenting a maximum peak at the instant when the vortex and cylinder are centered (about $t=0.0042 \mathrm{~s}$ ). Overall opposite to the time-varying lift coefficient the magnitude of the drag coefficient does not follow any pattern as a function of the vortex-cylinder horizontal miss-distance.

Although from the present investigations it was found that the Strouhal number decreases with the decrease of the vortex-cylinder horizontal miss-distance, a further analysis shows that the vortex-cylinder mechanism of interaction is very sensitive to the instant time 
of interaction. This following analysis focuses on the most critical instants in time of vortexcylinder interaction. The flow field was initialized from a resolved solution of uniform flow past circular cylinder at three different instants of time, these instants corresponding to a minimum, zero and respectively maximum lift coefficient values, considered to be the most critical ones from the mechanism of interaction point of view. In other words, the vortex will encounter the cylinder at different phase shifts, when the lifting coefficient is concerned.

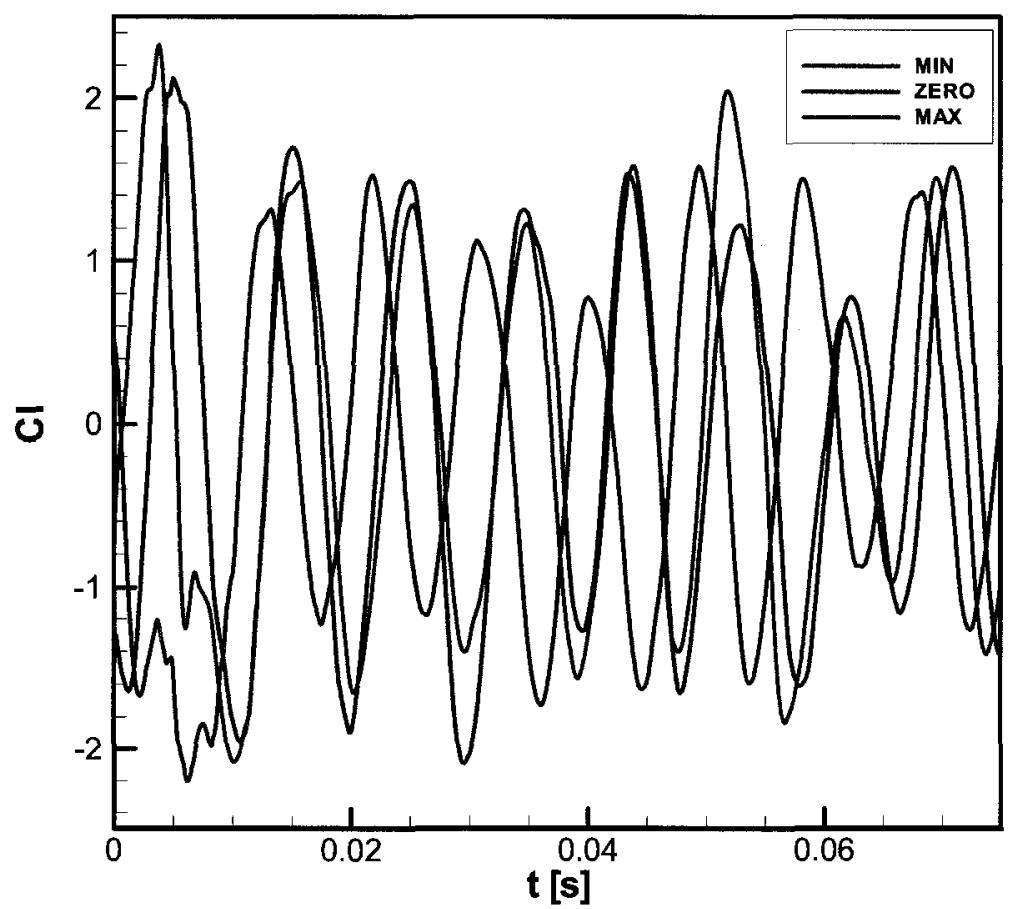

Figure 4.25 Superimposed clockwise rotating vortex on uniform flow past cylinder $(a=-1.5 D)$, lift coefficient comparison. 


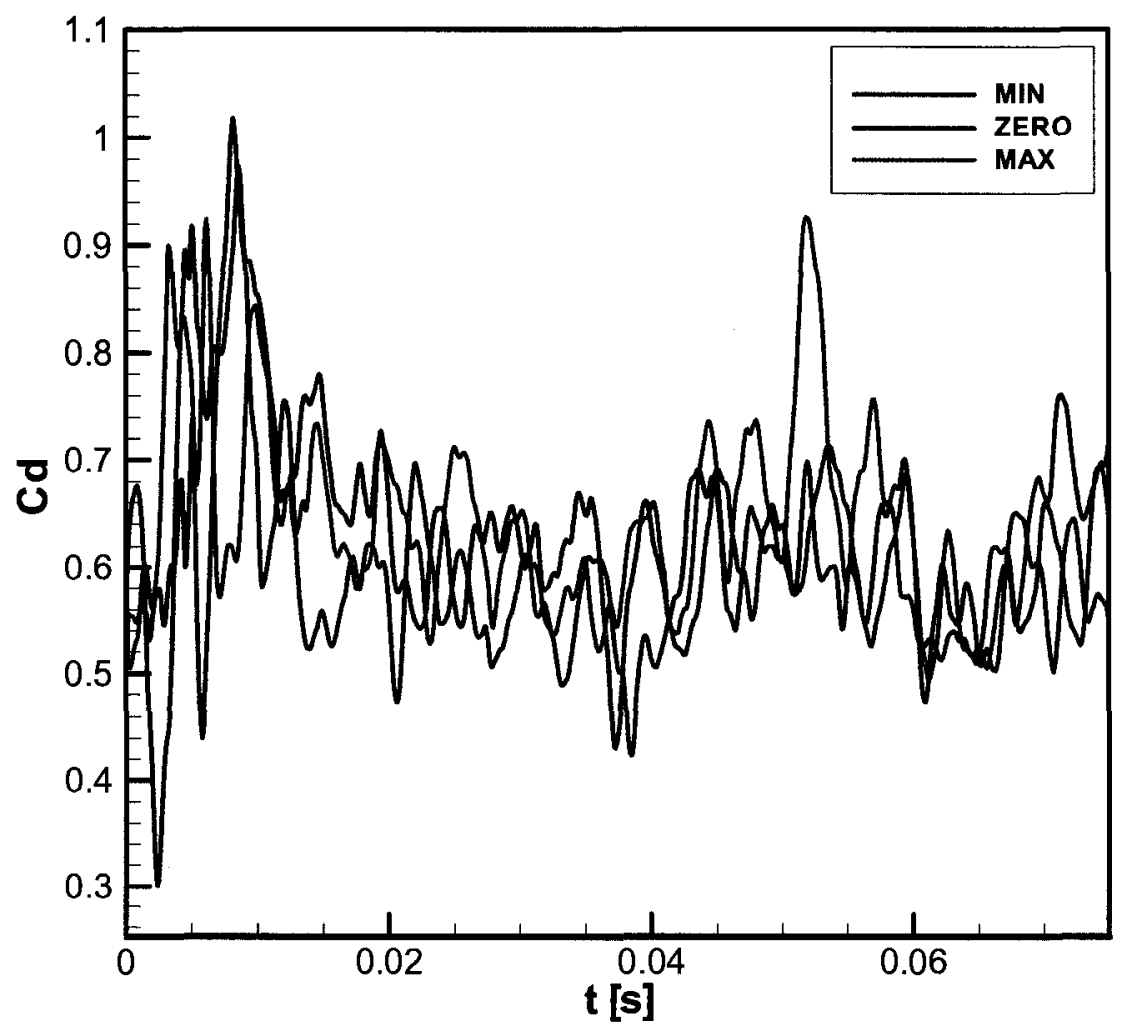

Figure 4.26 Superimposed clockwise rotating vortex on uniform flow past cylinder $(a=-1.5 D)$, drag coefficient comparison.

Due to computational reasons it was preferred that the flow field to be initialized at different instants in time, instead of releasing the vortex at different locations, since a large vortexcylinder miss-distance would require a high number of time steps in order to account for a fully developed interaction. Hence, a constant value of vortex-cylinder miss-distance was used for all three test cases, $\mathrm{a}=-0.315 \mathrm{~m}$. The initialization was made such that the vortexcylinder interaction occurs when the lift coefficient from the resolved uniform flow past cylinder reaches a minimum, zero and respectively a maximum value. From this analysis it was found that the Strouhal number is slightly higher for the case when the vortex-cylinder 
interaction occurs at the instant when the resolved lift coefficient for uniform flow presents a zero value, as seen in Figure 4.25.

The Strouhal number values corresponding to a minimum, zero, and respectively maximum lift coefficient values, were found as being $\mathrm{S}_{\mathrm{tzero}}=0.259, \mathrm{~S}_{\mathrm{tmin}}=0.2261, \mathrm{~S}_{\mathrm{tmax}}=$ 0.245. These values of Strouhal number are explained by the fact that the vortex encounters the cylinder at instants when there is either a balance or unbalance of pressure at the upper and lower surface of the cylinder and hence the mechanism of interaction affects the developing of the flow field in the wake of the cylinder in a specific manner for each case.

From the present analysis it was observed that the vortex-cylinder mechanism of interaction influences the flow field and implicitly the aerodynamic coefficients and Strouhal number in particular manner. The instant of interaction is a key factor in the vortex-cylinder mechanism of interaction and cylinder wake flow field development.

The investigations of vortex-cylinder mechanism of interaction provide good preparatory insights toward the investigation of airfoil-vortex mechanism of interaction. The first useful information provided by this analysis is related to the suitability of LES to problems associated with vortex dominated flows. The analysis has proven that URANS is not a suitable approach for vortex dominated flows. A second useful insight opening the horizon of further investigation is the fact that the vortex-bluff body interaction depends on vortex characteristics and instant of interaction. The present investigation of vortex-cylinder mechanism of interaction represents an original and novel work in the field of fluid dynamics. The present analysis provides significant details and valuable results when the vortex induced vibrations are of interest. 


\section{Chapter 5: The Aerodynamics and Aeroacoustics of AVI}

\subsection{Introduction}

In the present work, simulations of high Reynolds number flows around a thin symmetric NACA0012 airfoil were performed using large-eddy simulation. The flow is assumed to be two-dimensional, with the vortex axis parallel to the span of the blade. The influence of the vertical miss-distance, angle of attack and vortex characteristics on the aerodynamic coefficients is also investigated.

\subsection{Governing Equations}

A finite-volume Navier-Stokes solver (CFX-11.0) was used for both RANS and LES simulations. In the following the results using RANS and LES approaches are presented. The numerical analysis was done by parallel computing on a cluster having 16 nodes.

\subsection{Computational Model}

In the present analysis, superimposed vortex on uniform flow past NACA0012 airfoil (as schematically presented in Figure 1.1) is investigated using large-eddy simulation. For the solution of the filtered Navier-Stokes equations, a second order conservative central- 
difference scheme is used. For the time advancement, a second-order accurate implementation using a backward implicit difference formula is used.

Quasi 3-D simulations (using a thin 3-D type B computational domain, see ANSYS CFX-11.0) were performed for $\operatorname{Re}=1.3 \times 10^{6}$ based on the chord of the airfoil. An airfoil with a chord $\mathrm{c}=0.2 \mathrm{~m}$ was centered in the computational domain, whose outer boundary is a square with the sides equal to $9 \mathrm{c}$ and a spanwise size of $0.1 \mathrm{c}$. For computational efficiency reasons, the computational domain was divided into 12 blocks. The computational domain consists of 2.6 millions grid points with a cluster of grid points around the airfoil and a grid expansion factor of 1.01. For all the computations in the present analysis, a time step of $1 \mathrm{x}$ $10^{-6}$ seconds was chosen. The time-step is determined with respect to the explicit timemarching scheme and temporal resolution requirement of LES (CFL $\leq 1)$. In the present investigations, a value of Courant-Friedrichs-Lewy (CFL) number smaller than unity is maintained throughout the domain and the entire simulation.

The core size of the vortex was set equal to $\mathrm{c} / 4$, where $\mathrm{c}$ is the chord of the airfoil and the strength of the vortex is $25 \%$ higher than the uniform free-stream velocity. The strength and core size of the vortex were defined based on previous observations. ${ }^{18}$ Six different test cases were investigated based on the vortex location $(h=0.00,-0.005,-0.01,-0.015,-0.02$, and $-0.05 \mathrm{~m}$ ). For all test cases the releasing location of the vortex is $\mathrm{x}=0.13 \mathrm{~m}$, upstream of the leading edge.

No slip boundary conditions were used at the airfoil wall. Free slip boundary conditions were used at the top and bottom walls with opening at the end of the computational domain. In the present analysis we are interested in computing the pressure 
coefficient, $c_{p}$, lift coefficient, $c_{l}$, drag coefficient, $c_{d}$. The definitions of the coefficients are repeated here due to clarity purposes. The pressure coefficient is given by:

$c_{p}=\frac{p-p_{\infty}}{\frac{1}{2} \rho_{\infty} V_{\infty}^{2}}$

where $p$ is pressure, and $p_{\infty}, V_{\infty}$, and $\rho_{\infty}$ are the free-stream pressure, velocity and density, respectively. The lift coefficient is defined as:

$c_{l}=\frac{L^{\prime}}{\frac{1}{2} \rho_{\infty} V_{\infty}^{2} c}$

where $L^{\prime}$ is the lift force per unit span, $c$ is the characteristic chord length. The drag coefficient is defined as:

$c_{d}=\frac{D^{\prime}}{\frac{1}{2} \rho_{\infty} V_{\infty}^{2} c}$

where $D^{\prime}$ is the drag force per unit span.

The sequence of computations is as follows:

1) First the flow field for the case of uniform flow past the airfoil is solved using the Reynolds-averaged Navier-Stokes equations;

2) Secondly the LES simulation for the case of uniform flow past the airfoil is initialized using the RANS solution results;

3) Once the flow is fully developed, the LES solution results are collected and used for the initialization of vortex superimposed on a uniform flow. Due to the heavy amount of the required computational load, parallel processing is used. 
Similar to the case of circular cylinder, two different computational domains namely A and B were analyzed, for the NACA 0012 airfoil. For both test cases a structured grid type was used. A schematic view of the two computational domains is presented in the following subsection.

\subsubsection{NACA0012 computational domain type A}

Figure 5.1 presents a schematic view of type A multi-block structured grid, showing the cluster of grid points at the airfoil surface. Similar to the case of circular cylinder type A, the present computational domain provides good results regarding the aerodynamic investigations. However, if the aeroacoustic analysis is of interest, the present computational domain would provide poor prediction of results; the walls having significant influence on the flow field.

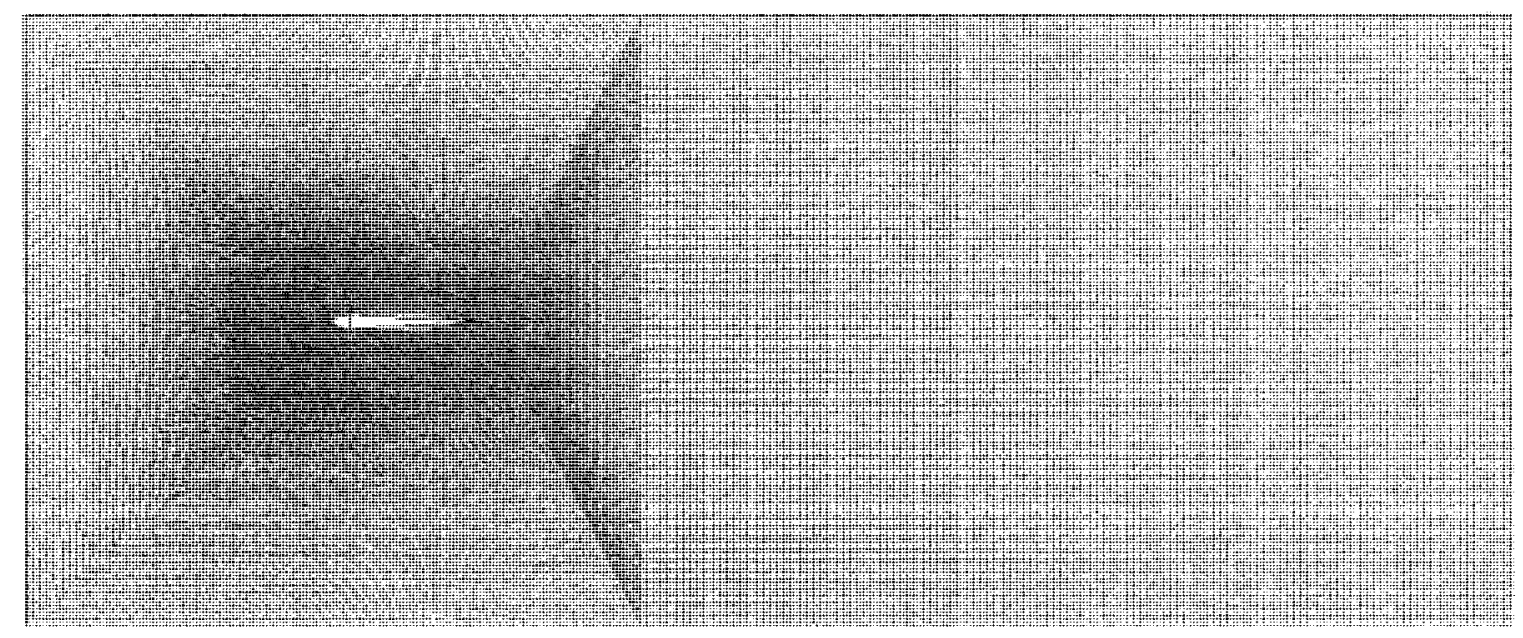

Figure 5.1 Schematic view of airfoil computational domain type A. 


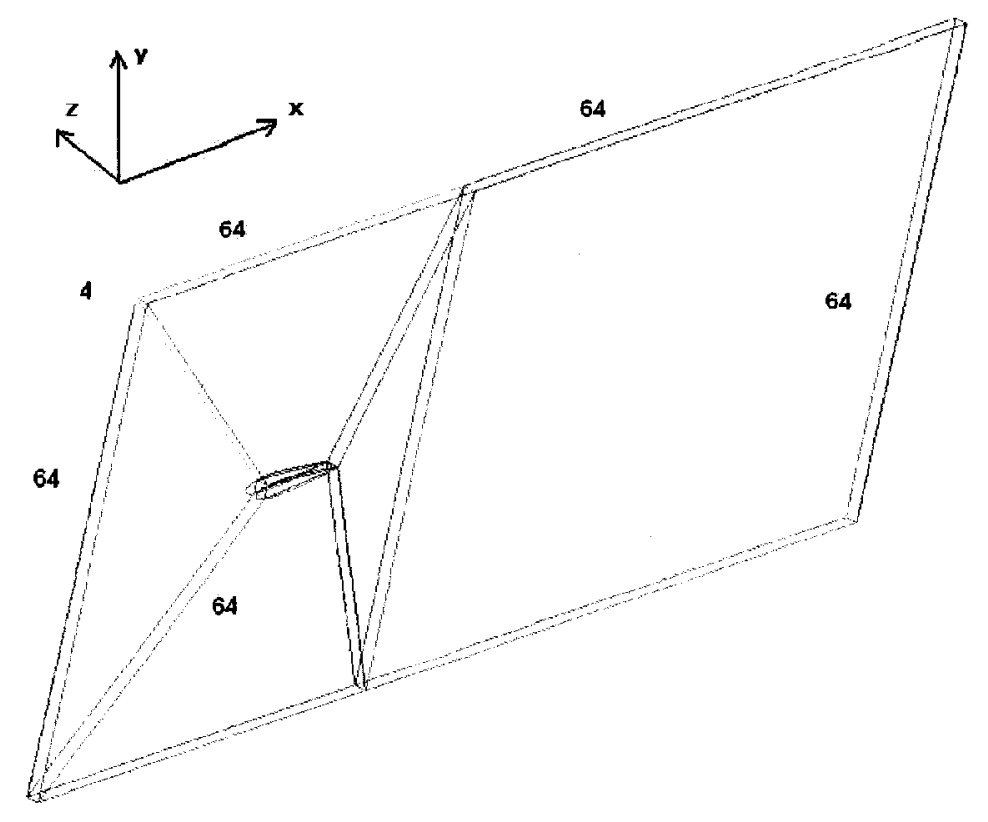

Figure 5.2 Schematic view of airfoil computational domain type A.

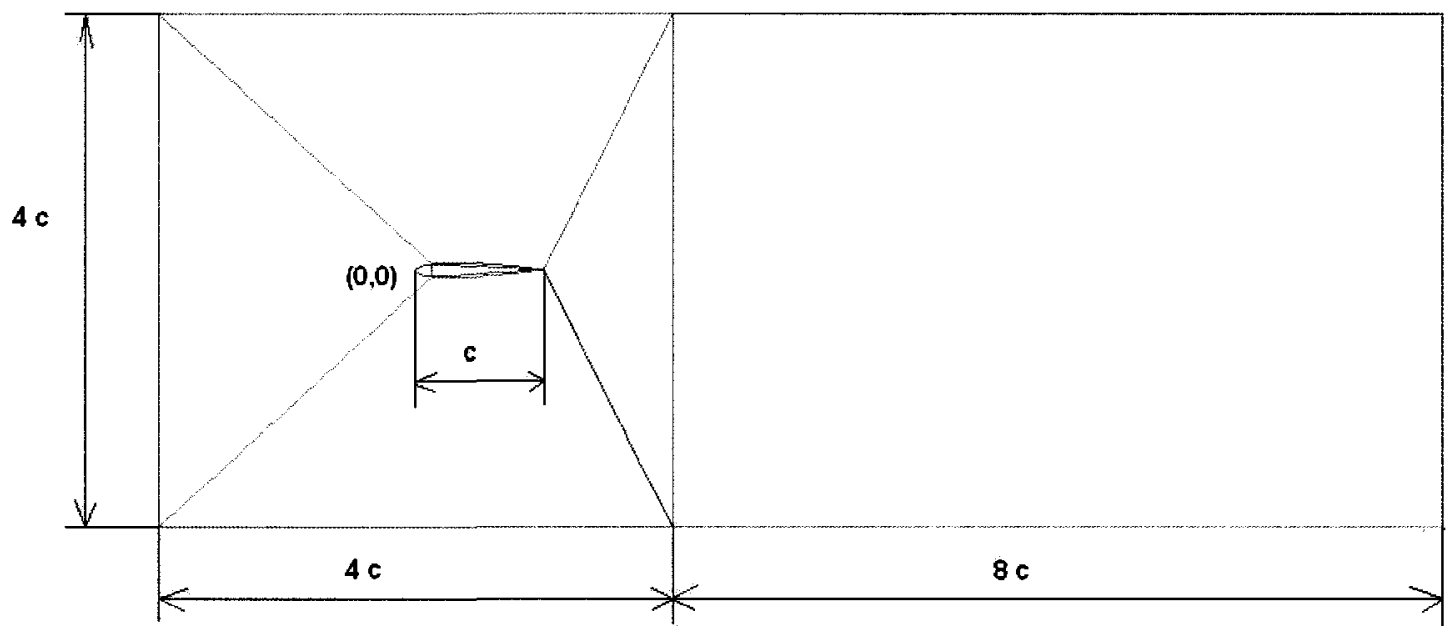

Figure 5.3 Schematic view of airfoil computational domain type A.

Figure 5.2 presents the schematic view of computational domain emphasizing the structure, location and number of grid points on each side of the computational domain. The computational domain consists of five blocks, the blocks surrounding the airfoil being part of 
a O-grid type. Figure 5.3 presents the dimensions of the computational domain. All the dimensions are based on the airfoil chord size. For the NACA 0012 airfoil, four different grid sizes were analyzed. The number of nodes in the z-direction was kept constant for all four grid sizes and only the number of nodes on the other sides was varied. The four grid sizes are presented in the Table 5.1.

Table 5.1 NACA 0012 airfoil type A grid sizes

\begin{tabular}{|c|c|c|c|}
\hline Case \# & Location of the first node from the wall & Expansion factor & Number of nodes \\
\hline 1 & 0.014 & 1.01 & 80,640 \\
\hline 2 & 0.007 & 1.01 & 325,120 \\
\hline 3 & 0.0035 & 1.01 & $1,305,600$ \\
\hline 4 & 0.002 & 1.01 & $2,613,472$ \\
\hline
\end{tabular}

\subsubsection{NACA0012 computational domain type B}

Similar to the case of circular cylinder, a type B computational domain was investigated for the NACA0012 airfoil. In this sense, a larger computational domain was used, the walls being located far away from the airfoil geometry. Four different grid sizes were also investigated for the case of NACA0012 airfoil as it is presented in Table 5.2. Figure 5.4 presents a schematic view of type B multi-block, structured grid computational domain. From Figure 5.4, a cluster of grid points near the airfoil surface can be identified. 


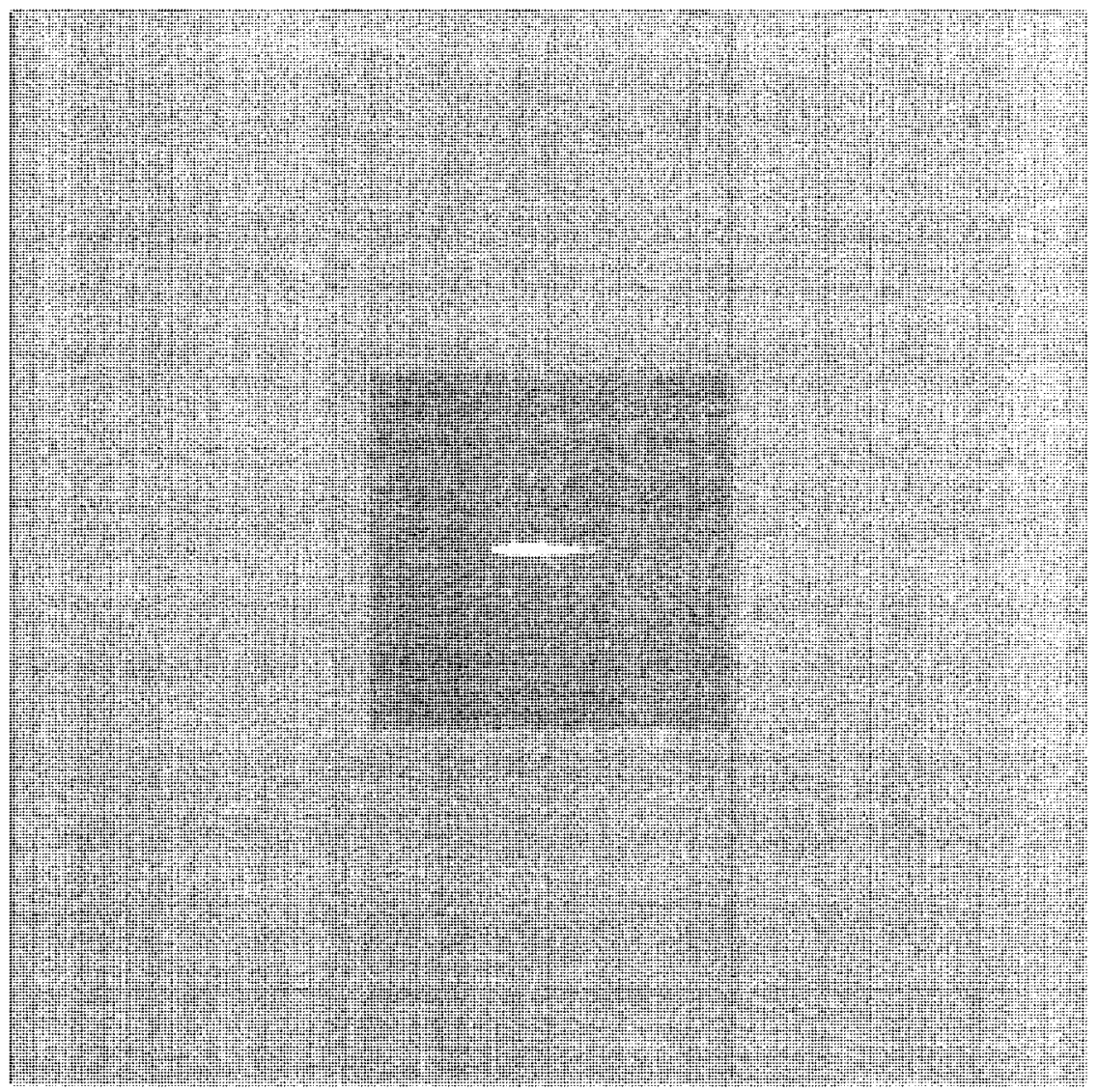

Figure 5.4 Schematic view of NACA0012 airfoil computational domain, type B.

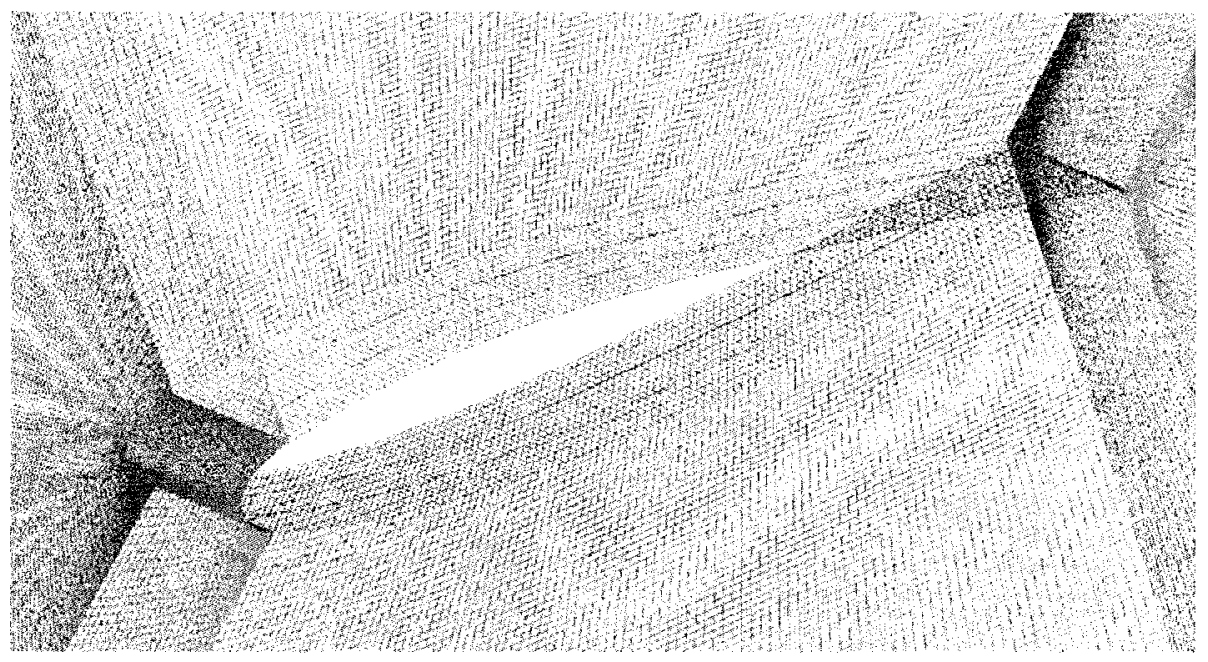

Figure 5.5 Schematic close-view of NACA0012 airfoil computational domain, type B. 


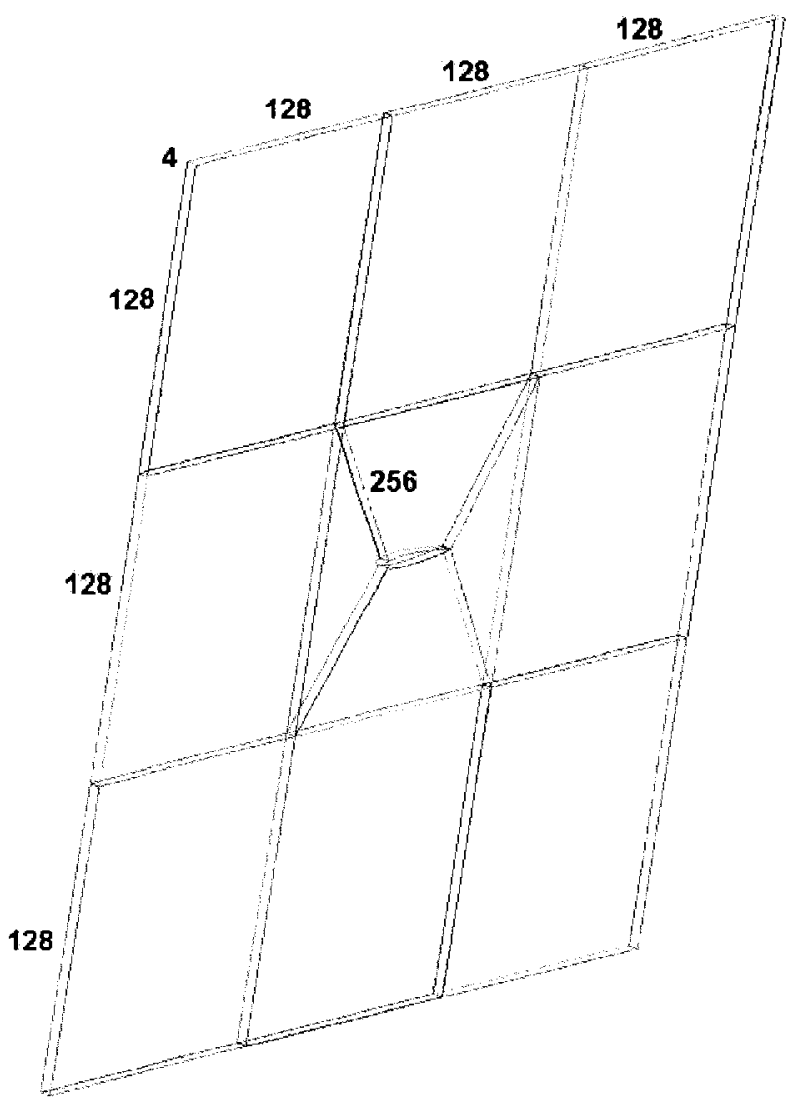

Figure 5.6 Schematic view of NACA0012 airfoil, computational domain type B. 


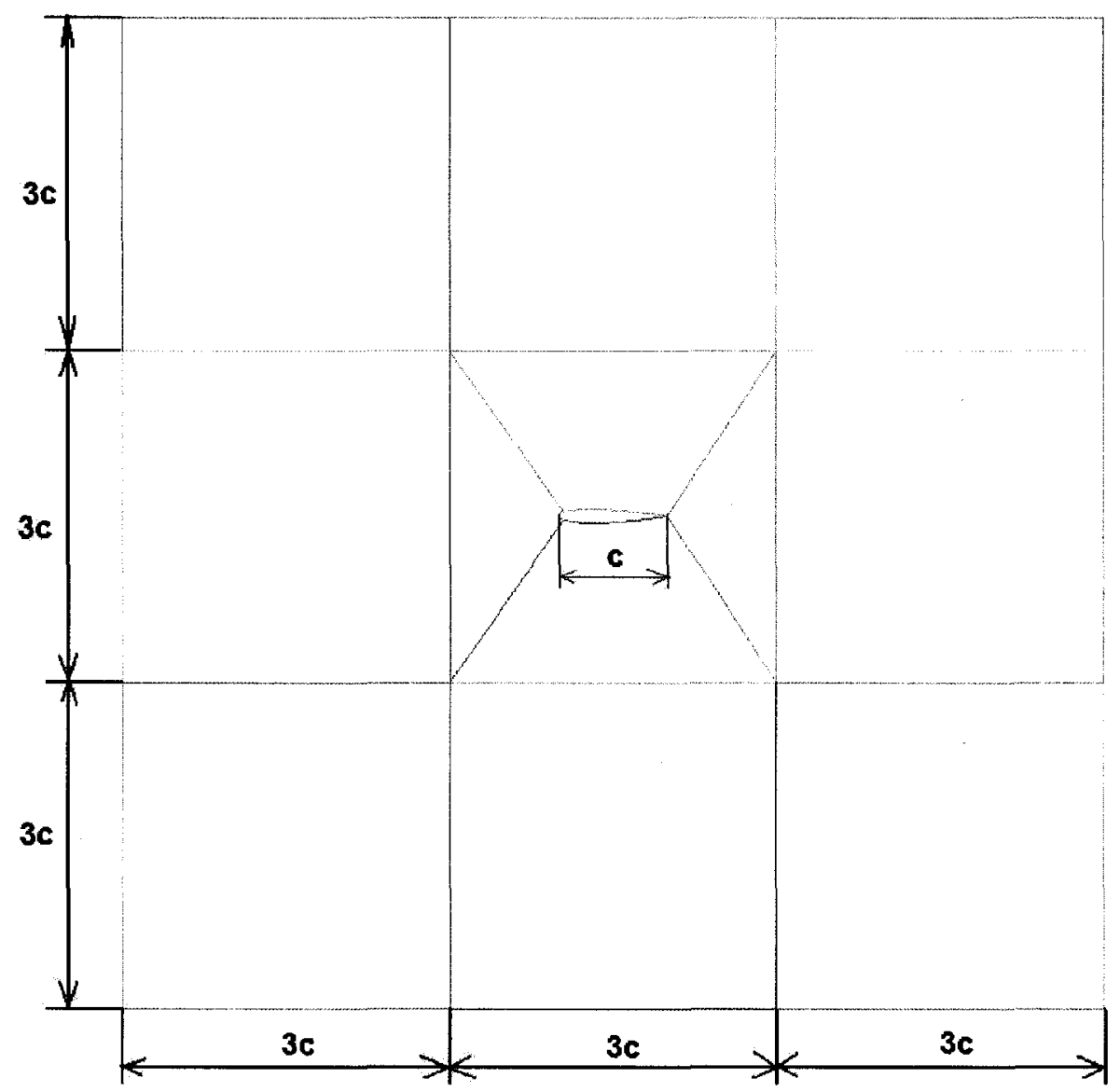

Figure 5.7 Schematic view of NACA0012 airfoil, size of the computational domain.

Figure 5.5 presents a close of view of the grid size (near the airfoil surface). Figure 5.6 shows the distribution of the grid points along the sides of the computational domain. The computational domain consists of 12 blocks. The choice of multi-block computational domain was based on computational reasons. Figure 5.7 presents the schematic view of the size of computational domain. In Figure 5.7, $c$ represents the size of the chord of airfoil. The present computational domain provides very good results not only for the aerodynamic analysis but also for the aeroacoustic investigations. The size of computational domain is also of concern when the aerodynamics and aeroacoustics of airfoil at high angle of attack is 
investigated, since there are wake perturbations which might sense the influence of the walls. Also for NACA0012 airfoil, type B computational domain, four different grid sizes were also investigated as it is presented in Table 5.2.

Table 5.2 NACA 0012 type B, grid size

\begin{tabular}{|c|c|c|c|}
\hline Case \# & Location of the first node from the wall [mm] & Expansion factor & Number of nodes \\
\hline 1 & 0.0097 & 1.01 & 4.800 \\
\hline 2 & 0.0075 & 1.01 & 19,840 \\
\hline 3 & 0.0035 & 1.01 & 80,640 \\
\hline 4 & 0.0018 & 1.01 & 325,120 \\
\hline 5 & 0.0009 & 1.01 & $1,305,600$ \\
\hline
\end{tabular}

\subsubsection{Grid Convergence Analysis}

Similar to the circular cylinder test case, a grid convergence analysis was performed for NACA0012 airfoil geometry for a type B computational domain, based on five different grid sizes, using RANS approach. From the grid convergence analysis it can be seen that for type B grid, a number of about 80,000 nodes is sufficient for accurate RANS results. The reason of choosing a denser grid was based on LES requirements, where a finer grid would provide better results, in terms of accuracy. The grid convergence analysis for type B computational domain is presented below. Similar to the case of circular cylinder the largest variation in velocity and pressure field occurs in vertical and respectively horizontal direction. Hence the interrogation lines are chosen accordingly, corresponding to the direction of largest variation. 


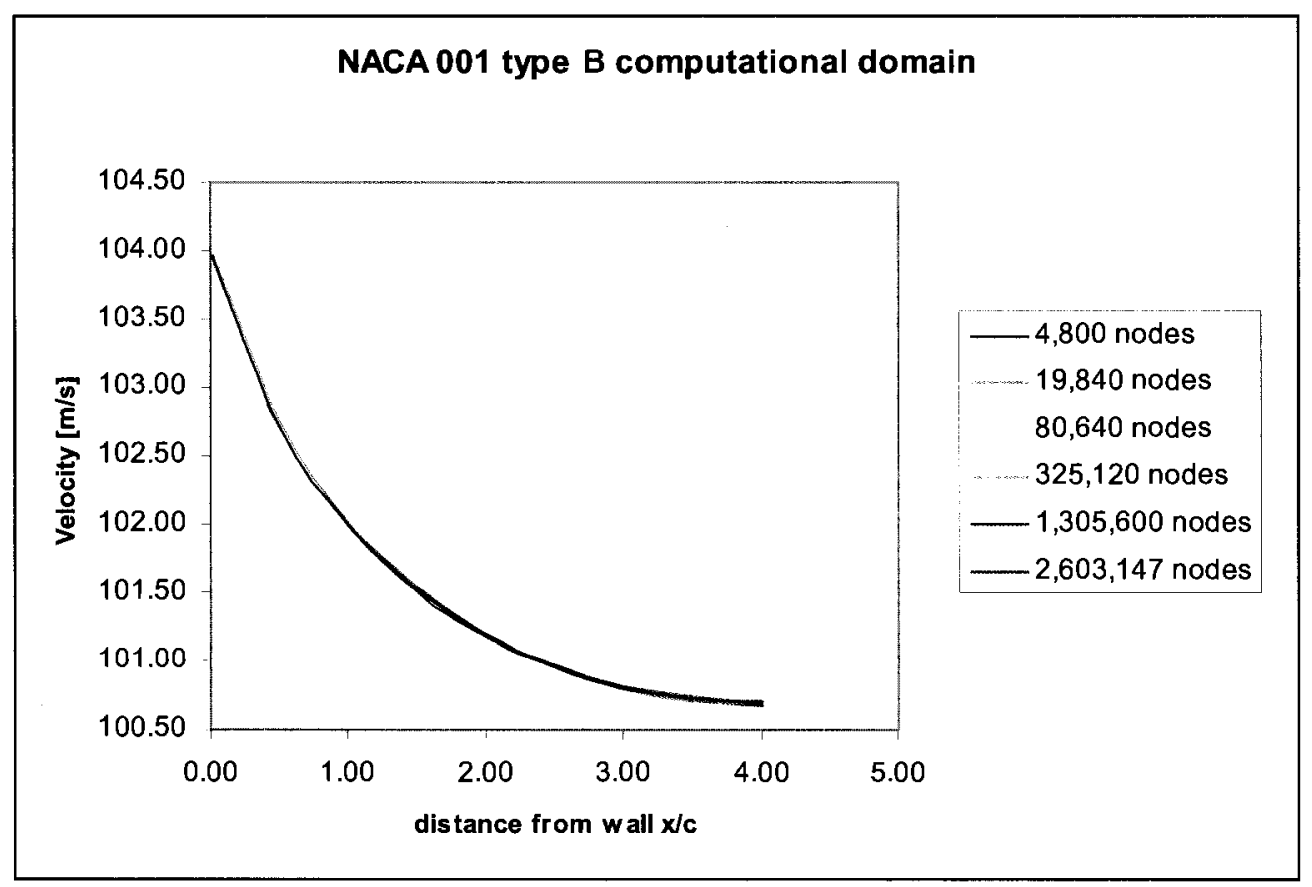

Figure 5.8 Grid convergence analysis

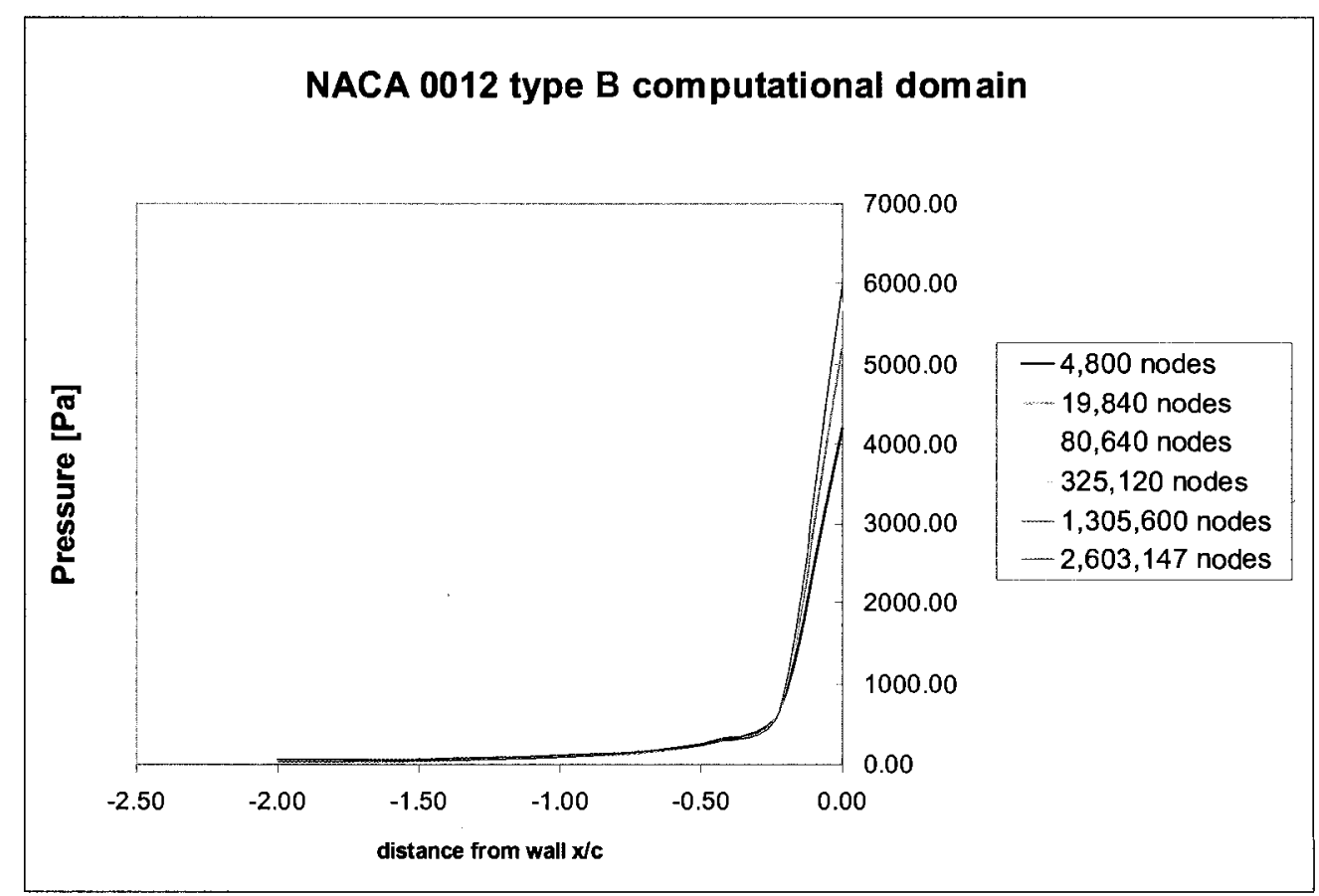

Figure 5.9 Grid convergence analysis 


\subsection{Aerodynamic Results (Airfoil at $0^{\circ}$ Angle of Attack)}

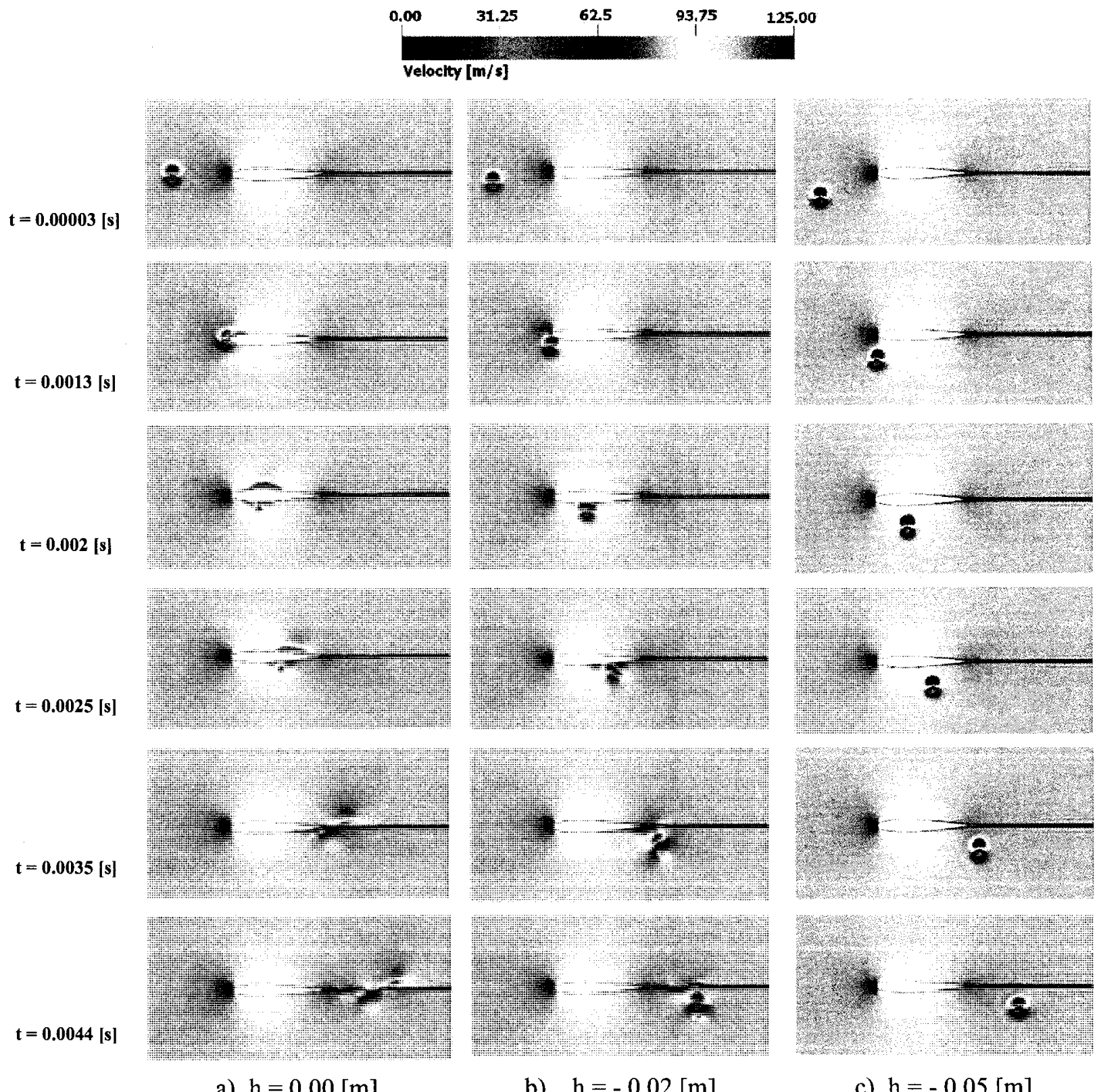
a) $\mathrm{h}=0.00[\mathrm{~m}]$
b) $\mathrm{h}=-0.02[\mathrm{~m}]$
c) $h=-0.05[\mathrm{~m}]$

Figure 5.10 Time evolved fluid structures. 


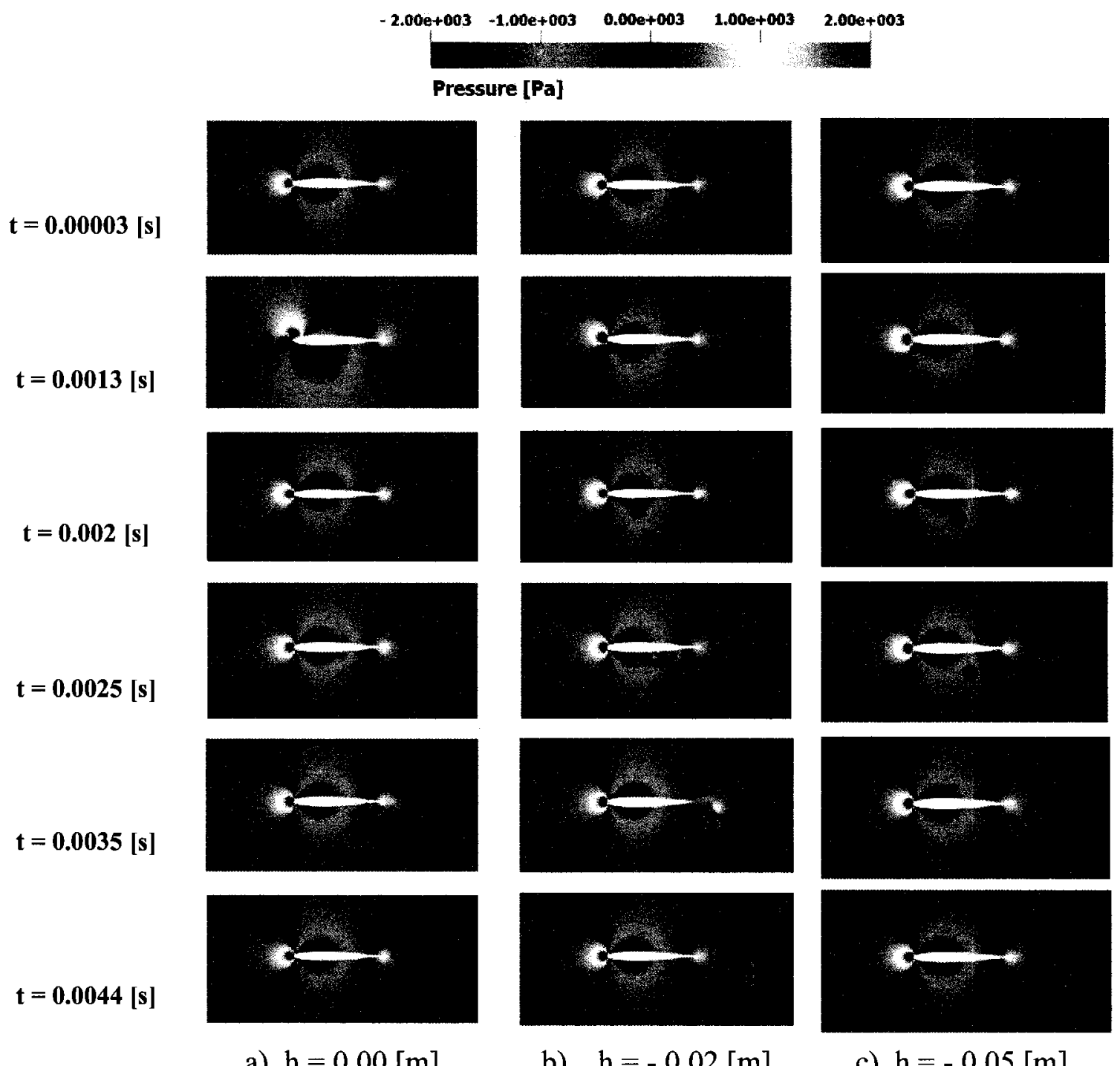
a) $\mathrm{h}=0.00[\mathrm{~m}]$
b) $\mathrm{h}=-0.02[\mathrm{~m}]$
c) $\mathrm{h}=-0.05[\mathrm{~m}]$

Figure 5.11 Time evolved fluid structures, pressure magnitude.

Figures 5.10 and 5.11 shows the LES results of instantaneous magnitude of velocity and pressure, respectively, for three different test cases based on the vertical miss-distance $h$, at six different instants in time as separate clockwise rotating vortices travel downstream.

The first left column in Figure 5.10 presents the airfoil-vortex mechanism of interaction for the case of a vertical miss-distance $h=0.00 \mathrm{~m}$. A good insight into the airfoil- 
vortex mechanism of interaction can be gained from the time history of velocity field, as it follows. The airfoil-vortex mechanism of interaction can be described in three different phases. At the start of the BVI, the vortex is at upstream location and moving toward the airfoil leading edge, as shown in Figure 5.10. At this location the vortex induces a downwash on the airfoil and causes the leading-edge stagnation point to move along the upper surface. This results into an increase in pressure over the airfoil upper surface. In a second phase, after the vortex passes the leading edge, it induces an upwash on the airfoil. This causes the stagnation point to move from the upper surface to the leading edge and then toward the lower surface. This movement of the stagnation point affects the lift on the blade. The rate of change of lift is related to the pressure propagated to an observer. As the airfoil-vortex mechanism of interaction progresses, the vortex is distorted while traveling the chord length of the airfoil. In the third phase, the remaining part of the vortex leaves the airfoil and wake perturbations are generated, as seen in Figure 5.10.

The second column shows the time history for a vertical miss-distance $h=-0.02 \mathrm{~m}$. It reveals the fact that the vortices interact progressively more with the lower surface of the airfoil as the miss-distance is increased, forming a unique and modified "dual-core" vortex (two clockwise/counter-clockwise or counter-rotating vortices connected together) as seen at instants of time of 0.0035 and $0.0044 \mathrm{~s}$ (for both velocity and pressure contours). At a higher miss-distance of $h=-0.05 \mathrm{~m}$, the vortex travels nearly undisturbed, but still interacting with the distant airfoil, as discussed later. In the pressure contour plots and except for $\mathrm{h}=-0.05 \mathrm{~m}$, it is clearly seen that when the vortex encounters the airfoil at instant $t=0.0013 \mathrm{~s}$, the vortex will create a higher pressure zone at the top surface and a lower pressure zone at the bottom 
surface (both near the leading edge), causing counter-clockwise moments (or a leading-edge or "nose down" effect).

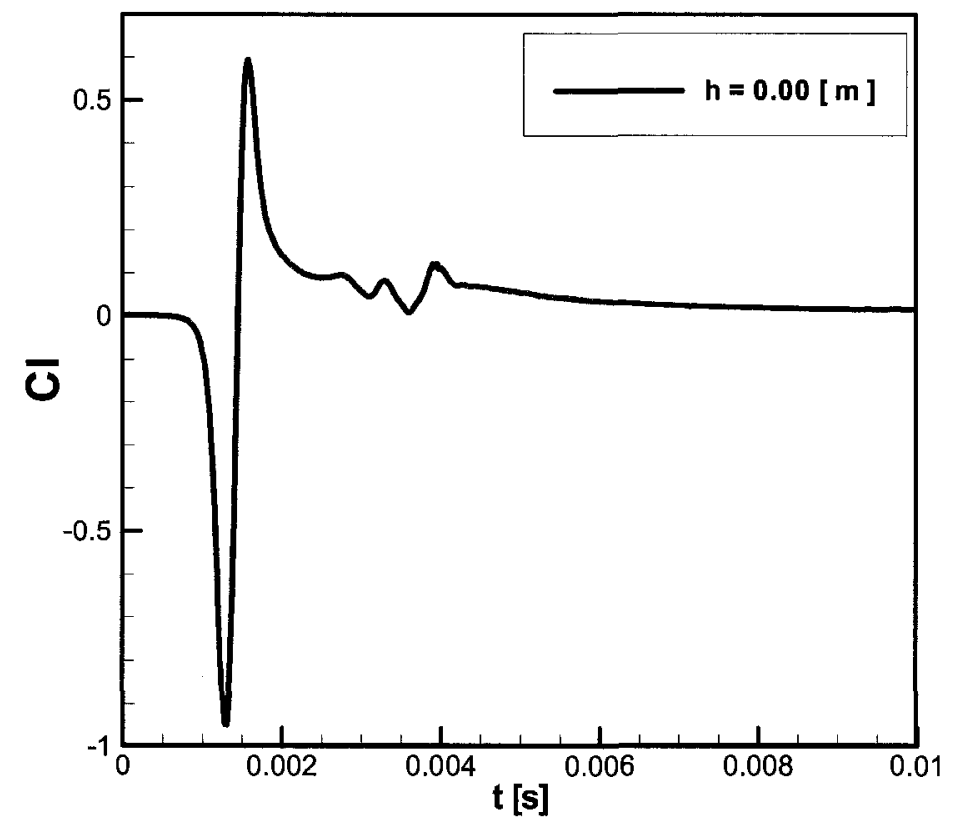

Figure 5.12 Time history of lift coefficient

Figure 5.12 shows the temporal variation of lift coefficient for a vortex superimposed on a uniform flow encountering a NACA 0012 airfoil at angle of attack $\alpha=0^{\circ}$ and a vertical missdistance $\mathrm{h}=0.00 \mathrm{~m}$. The lift coefficient is influenced by the pressure distribution over the airfoil surface. From Figure 5.12, it can be seen that there is a continuous decrease in lift as the vortex approaches the airfoil (until a minimum value in lift is achieved), followed by a sudden jump as the vortex core passes the airfoil leading edge. This sudden jump in lift coefficient is generated by a sudden change in the pressure coefficient. The sudden change in pressure coefficient has an aeroacoustic meaning and is associated with Blade-Vortex Interaction (BVI) noise, when the vortex encounters the airfoil. Although there is an increase 
of lift coefficient value when the vortex passes the leading edge, the absolute value of this maximum is smaller than the absolute minimum value. This difference in minimummaximum absolute values is due to the vortex core distortion when the vortex encounters the airfoil. When the residual component of the distorted vortex is far downstream from the airfoil, the lift coefficient converges asymptotically to a zero value and the flow field, in the vicinity of the airfoil, is almost undisturbed. Although the amplitudes of the lift coefficient are high for a zero vertical miss-distance, the fluctuation changes in lift coefficient are small when the vortex travels the chord length of the airfoil.

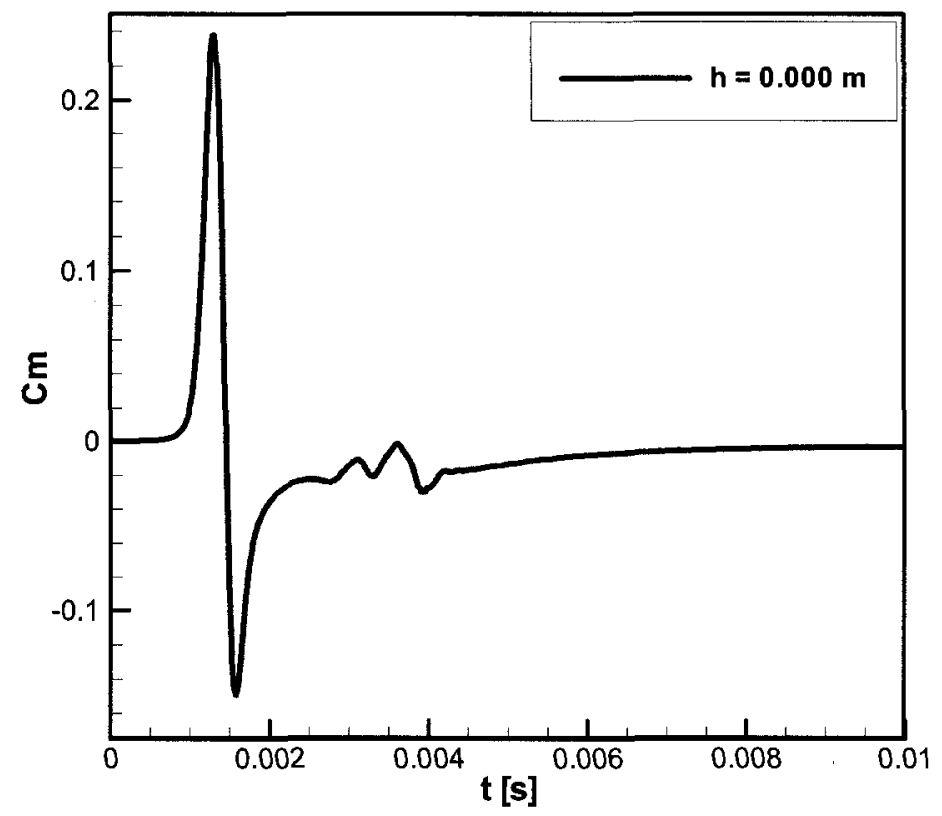

Figure 5.13 Time history of moment coefficient.

Figure 5.13 shows the time history of moment coefficient for a vertical miss-distance $h=$ $0.00 \mathrm{~m}$. The moment coefficient was calculated about $1 / 4$ of the chord length (aerodynamic center). The moment coefficient is considered positive in the counter clockwise direction. 
Notice that the moment coefficient has an inverted (mirrored) trend when compared against the lift coefficient. It can be seen that for the case of a clockwise rotating vortex, as the vortex approaches the airfoil, there is a positive moment corresponding to nose down. In the leading edge neighborhood for all the test cases, the moment coefficient shows the same behaviour. It follows that as the vortex encounters the airfoil, there is a sudden change in moment coefficient from positive to negative value as the center of the vortex moves downstream due to the clockwise rotating vortex direction. The moment coefficient increases as the vortex approaches the airfoil due to the increase of induced velocity and pressure field around the airfoil. The moment coefficient has a maximum value when the center of the vortex is coaxial with the leading edge. Similar to the lift coefficient as the residual component of the vortex travels downstream the airfoil, the moment coefficient converges asymptotically to a zero value, Figure 5.13.

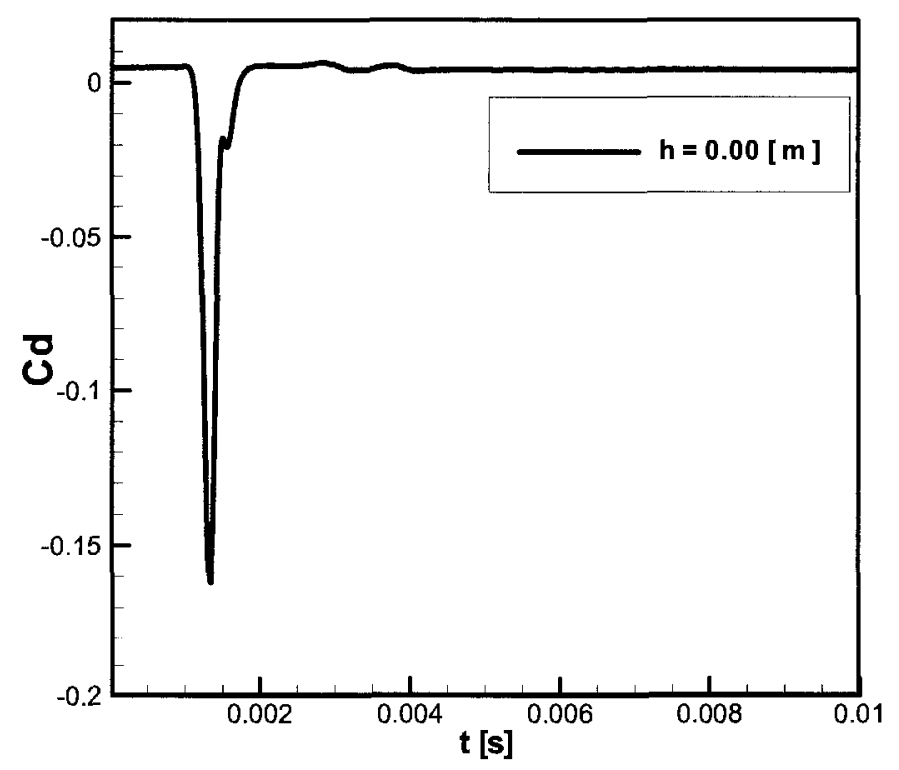

Figure 5.14 Time history of drag coefficient. 
For a Reynolds number of $1.3 \times 10^{6}$, the drag coefficient for a NACA 0012 airfoil at $0^{\circ}$ angle of attack is constant, $\mathrm{C}_{\mathrm{d}}=0.006$. The presence of the vortex in the flow field influences the drag coefficient as well. The temporal variation of drag coefficient for test case 1 ( $h=0.00$ $\mathrm{m})$ is presented in Figure 5.14. When the vortex and the airfoil are perfectly aligned, the vortex is distorted while interacting with the airfoil. The remaining part of the vortex traveling downstream along with the chord length of the airfoil causes separation at the airfoil surface and reverse flow, as indicated from the negative values of drag coefficient (at $t=$ $0.0015 \mathrm{~s})$. The airfoil-vortex mechanism of interaction has a significant impact on the drag coefficient as reflected by the large negative value of $\mathrm{C}_{d}=-0.16$. As the residual component of the vortex travels downstream the airfoil, the drag coefficient reaches a constant value of $\mathrm{C}_{\mathrm{d}}=0.006$.

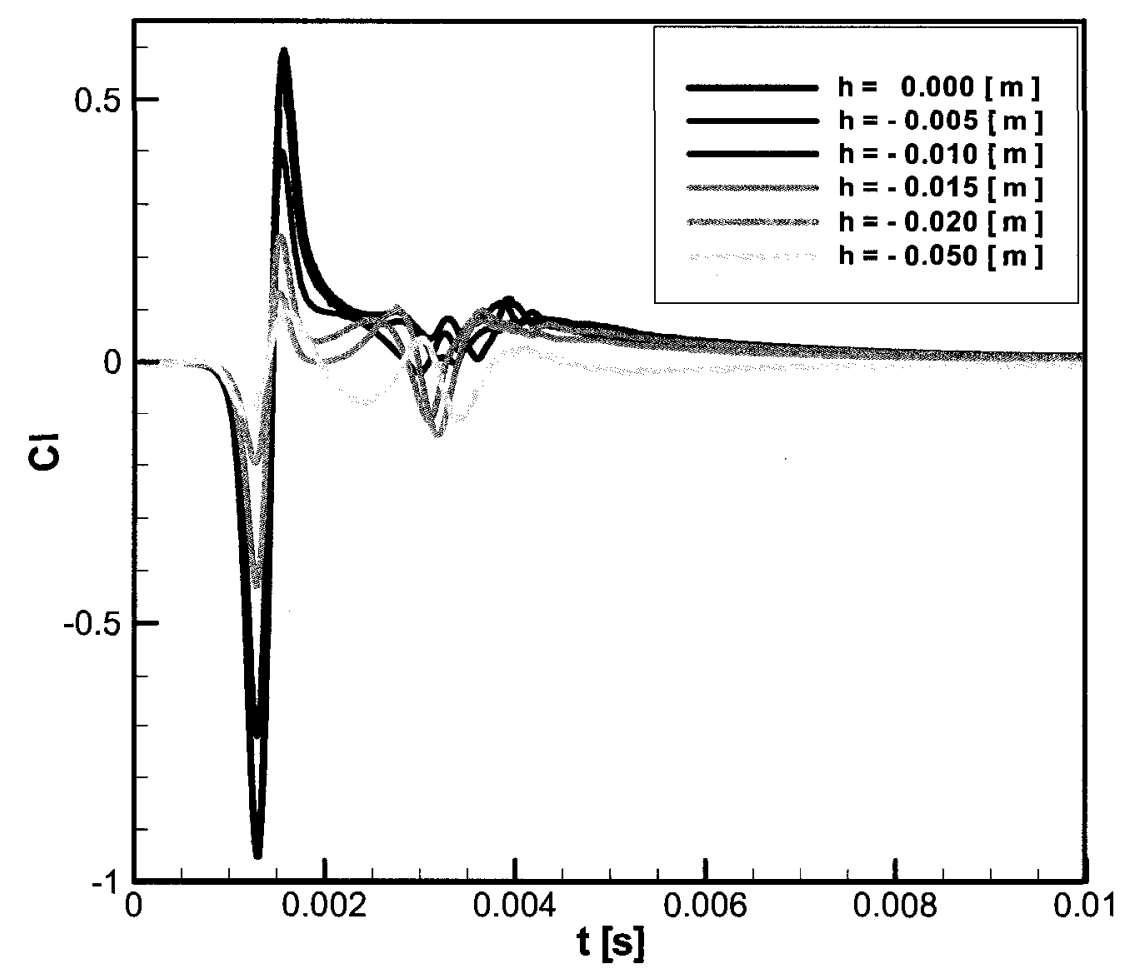

Figure 5.15 Comparison of lift coefficient. 
The comparison of aerodynamic coefficients, (i.e., the lift, moment, and drag coefficients) for different test cases, based on the airfoil-vortex miss-distance, is presented in Figures 5.15, 5.16 , and 5.17.

Figure 5.15 presents a comparison of lift coefficient for six different test cases, based on the airfoil-vortex vertical miss-distance (h). For the five test cases, the lift coefficient presents distinctive but similar patterns having three consecutive negative and positive peaks as clearly shown in Figure 5.15 for $\mathrm{h}=-0.02 \mathrm{~m}$.

The first negative and positive peak is associated with the incipient phase of airfoilvortex mechanism of interaction as the vortex approaches and interacts with the airfoil. By increasing the vertical miss-distance, $h$, the magnitudes of the first negative and positive peaks in lift coefficient decrease. This decrease is due to the fact that the airfoil-vortex interactions are less significant and have less impact on the flow field. The second positive lift coefficient peak (as the vortex passes half chord length at $t=0.0025 \mathrm{~s}$ ) is higher as the vertical miss-distance increases from $h=0.0$ to $h=-0.02 \mathrm{~m}$, except for $\mathrm{h}=-0.05 \mathrm{~m}$, where this second positive peak has a smaller value. The third peak in lift coefficient is achieved when the vortex leaves the airfoil. While the vortex is traveling away from the airfoil, the lift coefficient converges to zero from positive values for all cases, except for $\mathrm{h}=-0.05 \mathrm{~m}$. This leads us to the conclusion that the there is at least one single value of $h$ in the range of - 0.02 to $-0.05 \mathrm{~m}$ where the lift coefficient converges to zero at an early stage of the flow development. Notice that the lift coefficient is significantly changed even for $\mathrm{h}=-0.05 \mathrm{~m}$, where a nearly undisturbed vortex travels in a path relatively distant from the airfoil. 


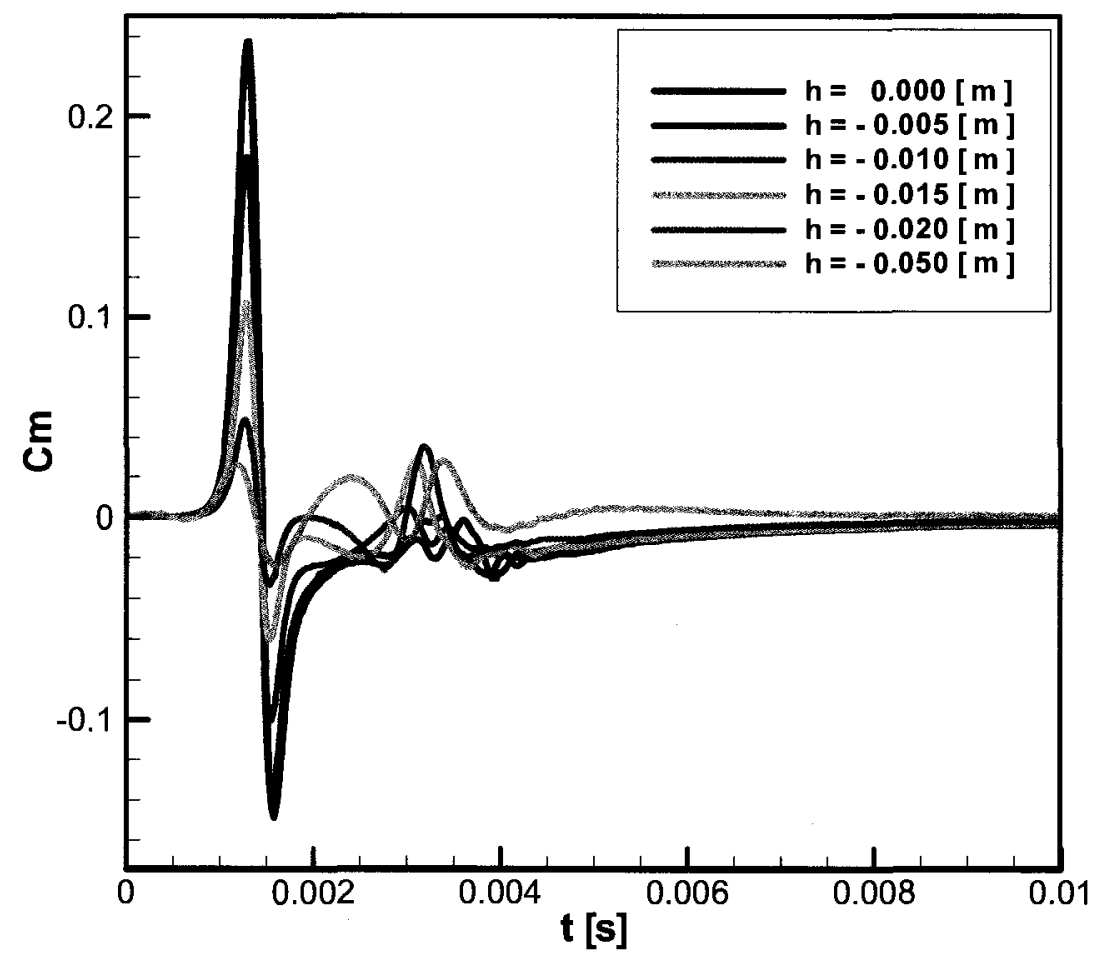

Figure 5.16 Comparison of moment coefficient.

For all test cases, an induced velocity pointing downwards is created at the airfoil surface as the vortex approaches the leading edge, causing a nose-down effect or positive moments as shown in Figure 5.16. 


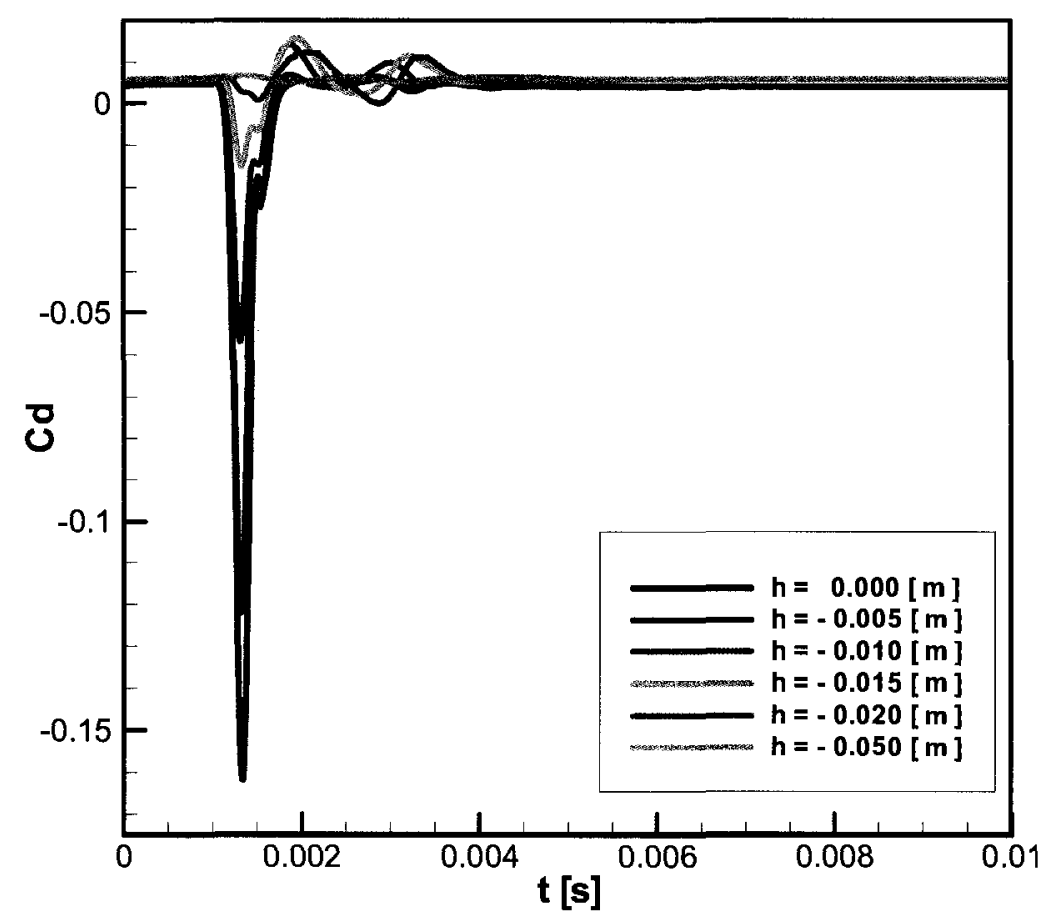

Figure 5.17 Comparison of drag coefficient.

Figure 5.17 presents the comparison of time-varying drag coefficient for five different test cases corresponding to five different vertical miss-distances $(h=-0.00,-0.01,-0.015,-$ 0.02 , and $-0.05 \mathrm{~m})$. For small vertical miss-distances $(\mathrm{h}=-0.00,-0.01,-0.015$, and -0.02 $\mathrm{m})$, large negative values of drag will be present as the vortex encounters the airfoil at about the instant $t=0.0013 \mathrm{~s}$. These negative values are caused primarily by separation and reverse flow over the surface of the airfoil. It can be seen that the most significant separation phenomenon occurs when the vertical miss-distance is small. As the vertical miss-distance increases, the airfoil-vortex interaction is less and less significant and likewise the separation, resulting in a decrease in magnitude of the negative peaks of drag coefficient. A small change in vertical miss-distance has a large impact on the drag coefficient at the instant when the 
vortex encounters the airfoil. Due to the airfoil-vortex mechanism of interaction, the distorted vortex causes fluctuations of drag coefficient as it travels the chord length of airfoil. The negative peaks of drag coefficient at the neighborhood of leading edge are followed by positive peaks at instants $t=0.0025 \mathrm{~s}$ and $\mathrm{t}=0.0035 \mathrm{~s}$, while the remaining part of the distorted vortex travels the chord length of the airfoil. As the residual component of the vortex leaves the airfoil, the drag coefficient reaches a constant value $C_{d}=0.006$. Increasing the vertical miss-distance to $h=-0.05 \mathrm{~m}$, the airfoil-vortex interaction is less severe and hence the drag coefficient sees a very small influence due to the presence of the vortex in the flow field as shown in the figure. 


\subsection{Aerodynamic Results (Airfoil at $5^{\circ}$ Angle of Attack)}

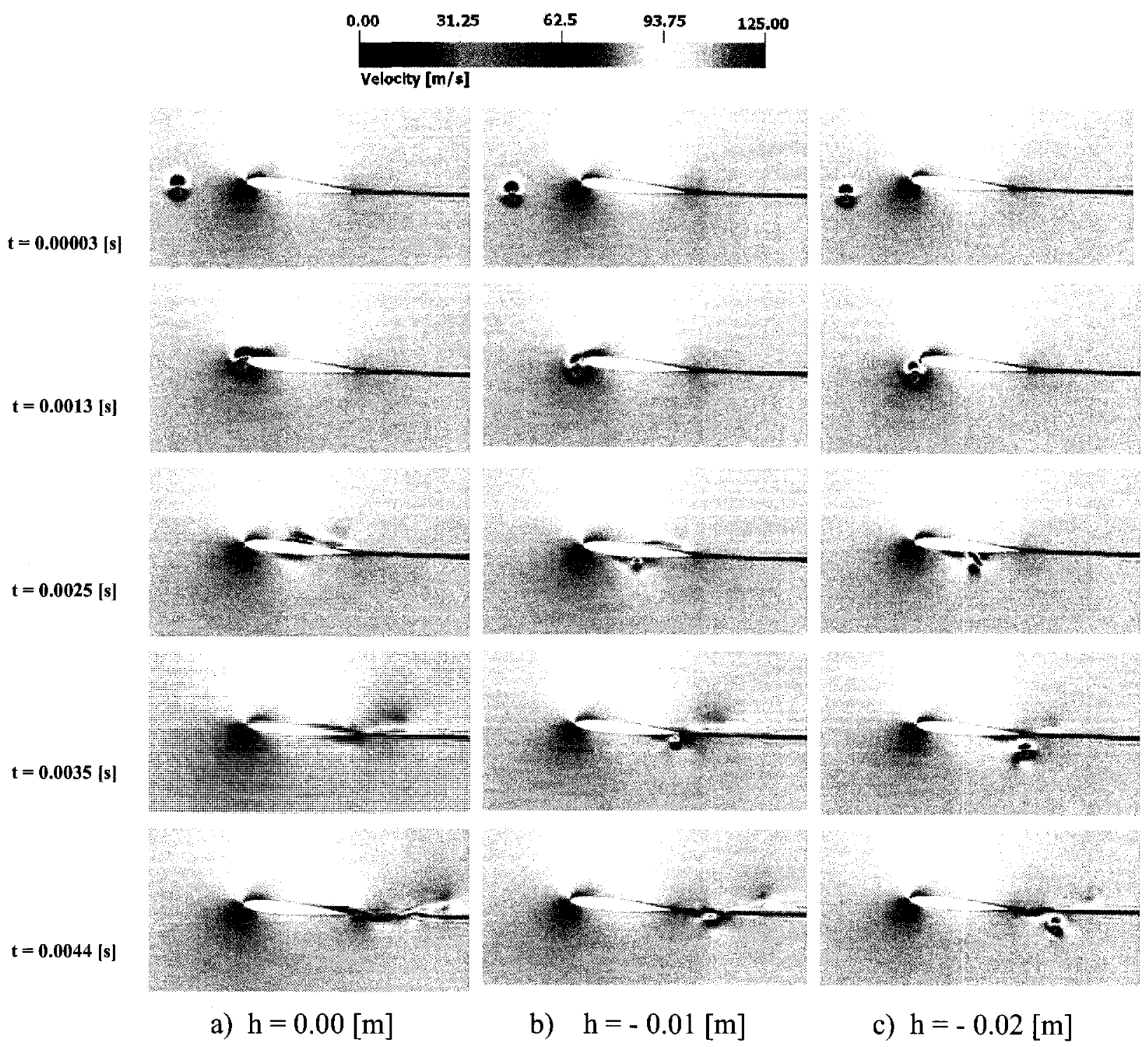

Figure 5.18 Time evolved fluid structures (velocity magnitude), angle of attack $\alpha=5^{\circ}$. 

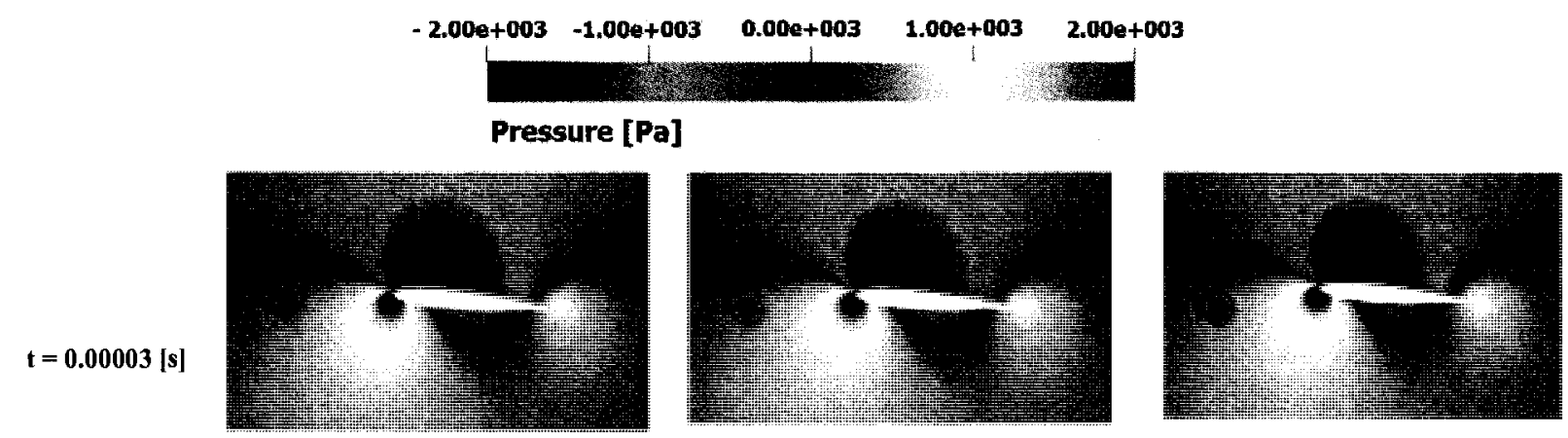

$\mathbf{t}=\mathbf{0 . 0 0 1 3}[\mathbf{s}]$
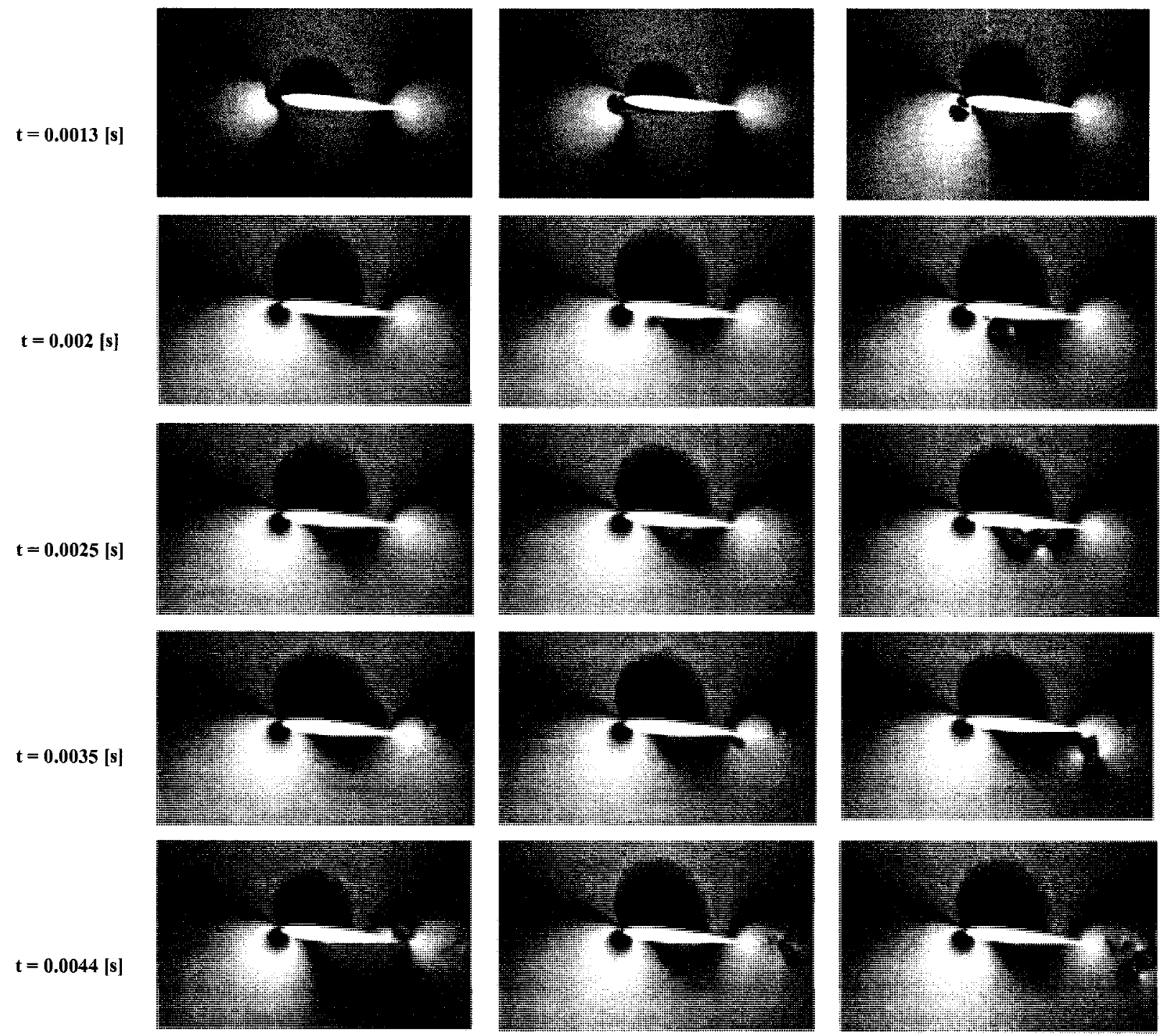

a) $\mathrm{h}=0.00[\mathrm{~m}]$

b) $\mathrm{h}=-0.01[\mathrm{~m}]$

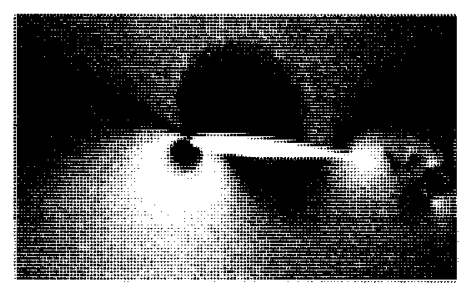

c) $\mathrm{h}=-0.02[\mathrm{~m}]$

Figure 5.19 Time evolved fluid structures (pressure magnitude), angle of attack $\alpha=5^{\circ}$. 
Similarly to the analysis of the airfoil at $0^{\circ}$ angle of attack, Figures 5.18 and 5.19 shows the LES results (at $5^{\circ}$ angle of attack) of instantaneous magnitude of velocity and pressure, respectively, for three different vertical miss-distances $(h=0.00,-0.01$, and $-0.02 \mathrm{~m})$ at six instants in time, for separate clockwise rotating vortices traveling downstream toward the airfoil.

For a vertical miss-distance $\mathrm{h}=0.00 \mathrm{~m}$, the airfoil-vortex mechanism of interaction is associated with distortion of the vortex core and an increase of velocity, as well large flow separation at the upper surface of airfoil as seen in Figure 5.18a. However, there is a significant amount of the remaining vortex traveling downstream and interacting with the airfoil wake and disturbing it.

Figure $5.18 \mathrm{~b}$ presents the velocity field for test case $\mathrm{h}=-0.01 \mathrm{~m}$. Similar to test case $\mathrm{h}=0.00 \mathrm{~m}$, the airfoil experiences an increase of velocity on the upper surface as the vortex encounters the airfoil. Due to the increase of airfoil-vortex vertical miss-distance the flow separation is less significant compared with test case $h=0.00 \mathrm{~m}$. As a result of airfoil-vortex mechanism of interaction, two vortices of different strengths are formed, traveling at considerable time difference, on two separate paths one above and below the airfoil wake. The vortex traveling above the airfoil wake has a higher strength than the one traveling below and this is also a result of airfoil-vortex mechanism of interaction. Figure $5.18 \mathrm{c}$ presents the time-varying velocity field corresponding to an airfoil-vortex vertical miss-distance $h=-$ $0.02 \mathrm{~m}$. From Figure $5.18 \mathrm{c}$, it can be seen that with the increase of vertical miss-distance the airfoil-vortex interactions become less and less severe and the vortex is less distorted, preserving its characteristics. The influence of vertical miss-distance on the airfoil-vortex mechanism of interaction is very well captured in Figure 5.18c. Hence the residual 
component of the distorted vortex traveling underneath of airfoil is defined by the large flow structure, as seen at instant $t=0.0025 \mathrm{~s}$. As the time elapse, the residual component of the vortex redefines a unique vortex, characterized by a smaller strength and core size, as seen the instant $\mathrm{t}=0.0044 \mathrm{~s}$.

Figure 5.19 presents the time-varying pressure field for an angle of attack $\alpha=5^{\circ}$, for three different vortex-airfoil vertical miss-distances. A large region of low pressure (negative pressure), at the upper surface of airfoil is observed for all test cases. For all test cases, large pressure vales are observed at the stagnation point. In this case the stagnation point is located on the lower surface of airfoil. The presence of the vortex in the flow field disturbs the pressure field generating pressure waves which are associated with the aeroacoustic noise, as it will be explained later on in this chapter. Similar to the time-varying velocity field, it can be seen that the vortex is completely distorted when the vertical miss-distance is small. For relatively larger vertical miss-distance values (e.g. $h=-0.02 \mathrm{~m})$ the vortex preserves better its characteristics, after the instant of interaction.

This result proves that depending on the airfoil-vortex mechanism of interaction the residual component of the vortex exhibits enough energy to recover and redefine a new unique flow structure. As the newly generated vortex travels far downstream, interacts with the airfoil wake and a merging process of the two occurs. As a result of the merging process, large wake disturbances are identified as well fast dissipation of the vortex.

The disturbance of the flow field by the presence of the vortex and the airfoil-vortex mechanism of interaction is reflected into the time variation of the aerodynamic coefficients (lift and drag) as presented in Figure 5.20 and Figure 5.21. The airfoil-vortex mechanism of interaction is a function of both, airfoil angle of attack and airfoil-vortex vertical miss- 
distance. The lift coefficient exhibits similar behaviour for the two angles of attack, $\alpha=0^{\circ}$ and $\alpha=5^{\circ}$, at the instant when the vortex encounters the airfoil $(\mathrm{t}=0.0013 \mathrm{~s})$.

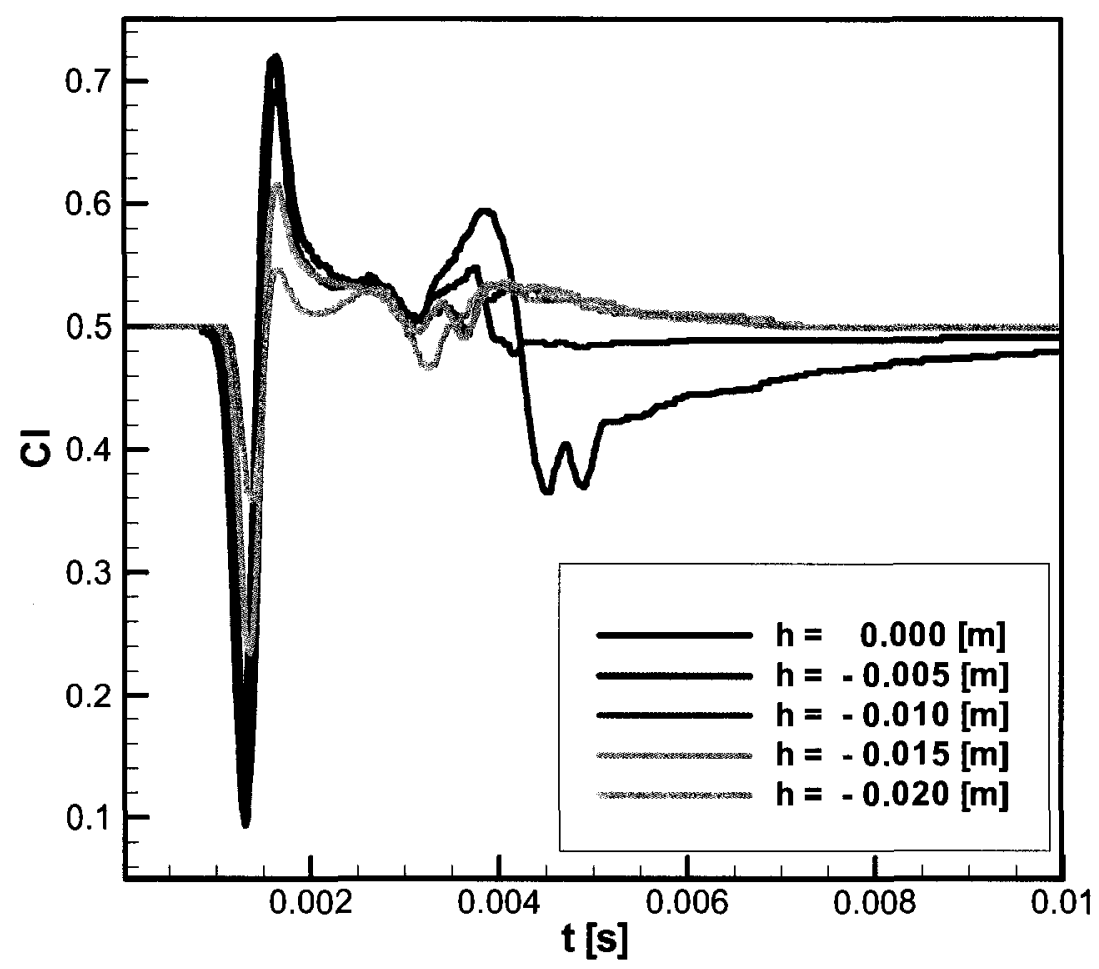

Figure 5.20 Comparison of lift coefficient, angle of attack $\alpha=5^{\circ}$.

Figure 5.20 presents a comparison of lift coefficient, based on the airfoil-vortex vertical missdistance for five different test cases for an angle of attack $\alpha=5^{\circ}$. From Figure 5.20, it can be seen that for all test cases, the lift coefficient presents similar behavior at the instant when the vortex encounters the airfoil, $t=0.0013 \mathrm{~s}$. However, the influence of airfoil-vortex vertical miss-distance on the mechanism of interaction is reflected in the magnitude of the lift coefficient amplitudes, at the instant when the vortex encounters the airfoil, as well in the fluctuations of lift coefficient as the vortex travels the airfoil chord length. As the airfoil- 
vortex vertical miss-distance increases, the amplitudes of lift coefficient at the instant when the vortex encounters the airfoil, decrease, instant $t=0.0013 \mathrm{~s}$. As a result of the airfoil-vortex mechanism of interaction, the fluctuations of lift coefficient (while the vortex travels the chord length of the airfoil) are more significant as the vertical miss-distance increases.

Large differences in lift coefficient values can be seen as the residual component of the distorted vortex leaves the airfoil and travels downstream, instant $t=0.004 \mathrm{~s}$. It is worth to notice that for the test cases $h=-0.01 \mathrm{~m}, \mathrm{~h}=-0.015 \mathrm{~m}$ and $\mathrm{h}=-0.02 \mathrm{~m}$, the lift coefficient exhibits very similar trend as the residual component of the distorted vortex leaves the airfoil and travels downstream.

From Figure 5.20, it can be seen that for small values of airfoil-vortex vertical missdistance, the lift coefficient converges to a constant value by an asymptotic growth, while for relatively large values of vertical miss-distance, the drag coefficient converges to constant value by an asymptotic decay. 


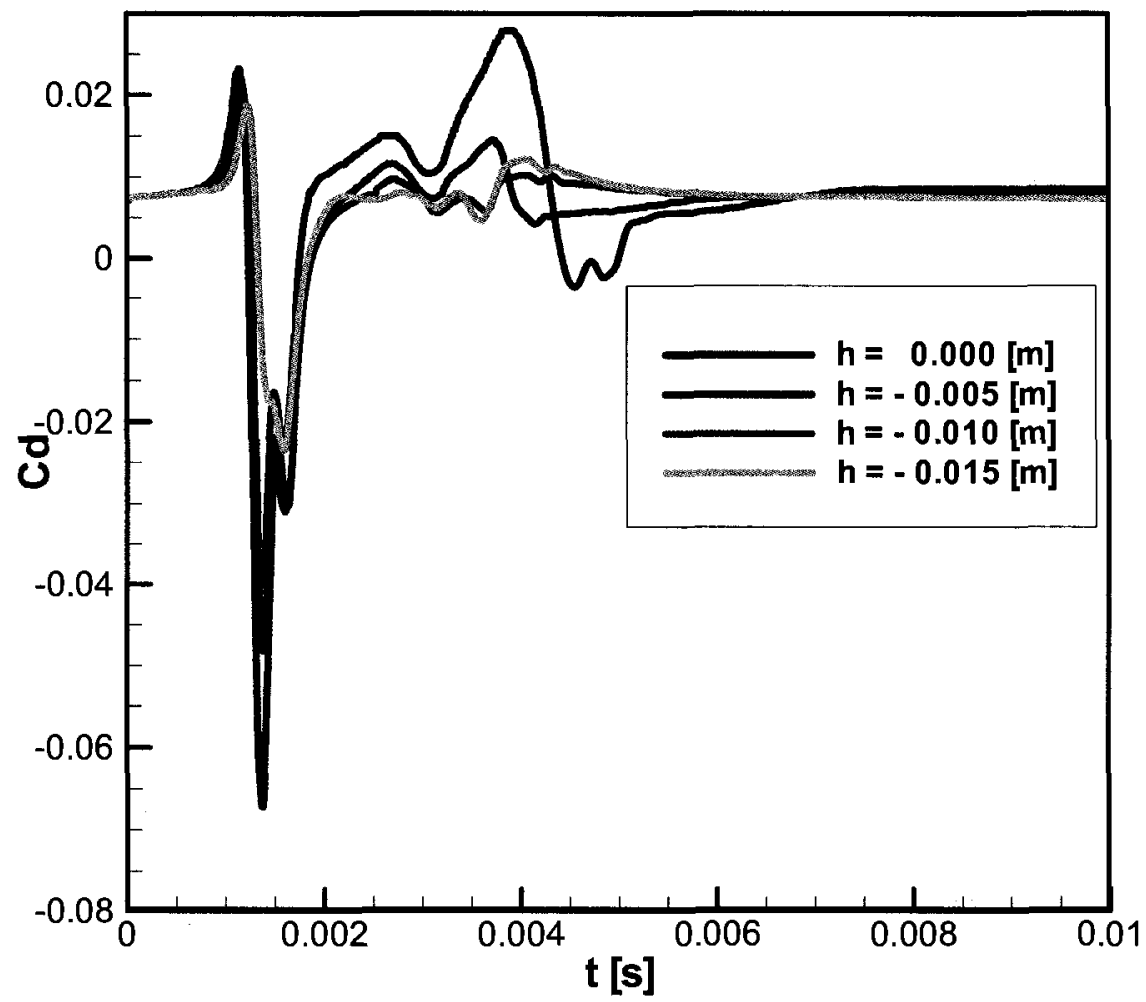

Figure 5.21 Comparison of drag coefficient, angle of attack $\alpha=5^{\circ}$.

Figure 5.21 presents the comparison of time-varying drag coefficient for an angle of attack $\alpha=5^{\circ}$, based on the airfoil-vortex vertical miss-distance h. For all test cases, large negative values of drag coefficient associated with reverse flow are observed at the instant when the vortex encounters the airfoil, $\mathrm{t}=0.0013 \mathrm{~s}$. For the case of angle of attack $\alpha=5^{\circ}$, there is not a direct relationship of drag coefficient function of vertical miss-distance as it was observed for the case of an angle of attack $\alpha=0^{\circ}$. For an angle of attack $\alpha=5^{\circ}$, the drag coefficient exhibits the largest negative value, associated with the reverse flow, for a vertical missdistance $h=-0.005 \mathrm{~m}$. This result is associated with the influence of angle of attack on the airfoil-vortex mechanism of interaction. For values of vertical miss-distance larger than $\mathrm{h}=-$ 
$0.01 \mathrm{~m}$, the interactions are less and less severe and likewise the separation, resulting in a decay in magnitude of the negative peaks of drag coefficient associated with reverse flow. For the case of a vertical miss-distance $\mathrm{h}=-0.005$, due to the mechanism of interaction reverse flow is observed at the instant when the residual component of the vortex travels downstream the airfoil, $\mathrm{t}=0.004 \mathrm{~s}$. As the vertical miss-distance increases, no reverse flow is observed; the vortex has the freedom to slide underneath of airfoil and the interactions are less severe. 


\subsection{Aerodynamic Results (Airfoil at $10^{\circ}$ Angle of Attack)}

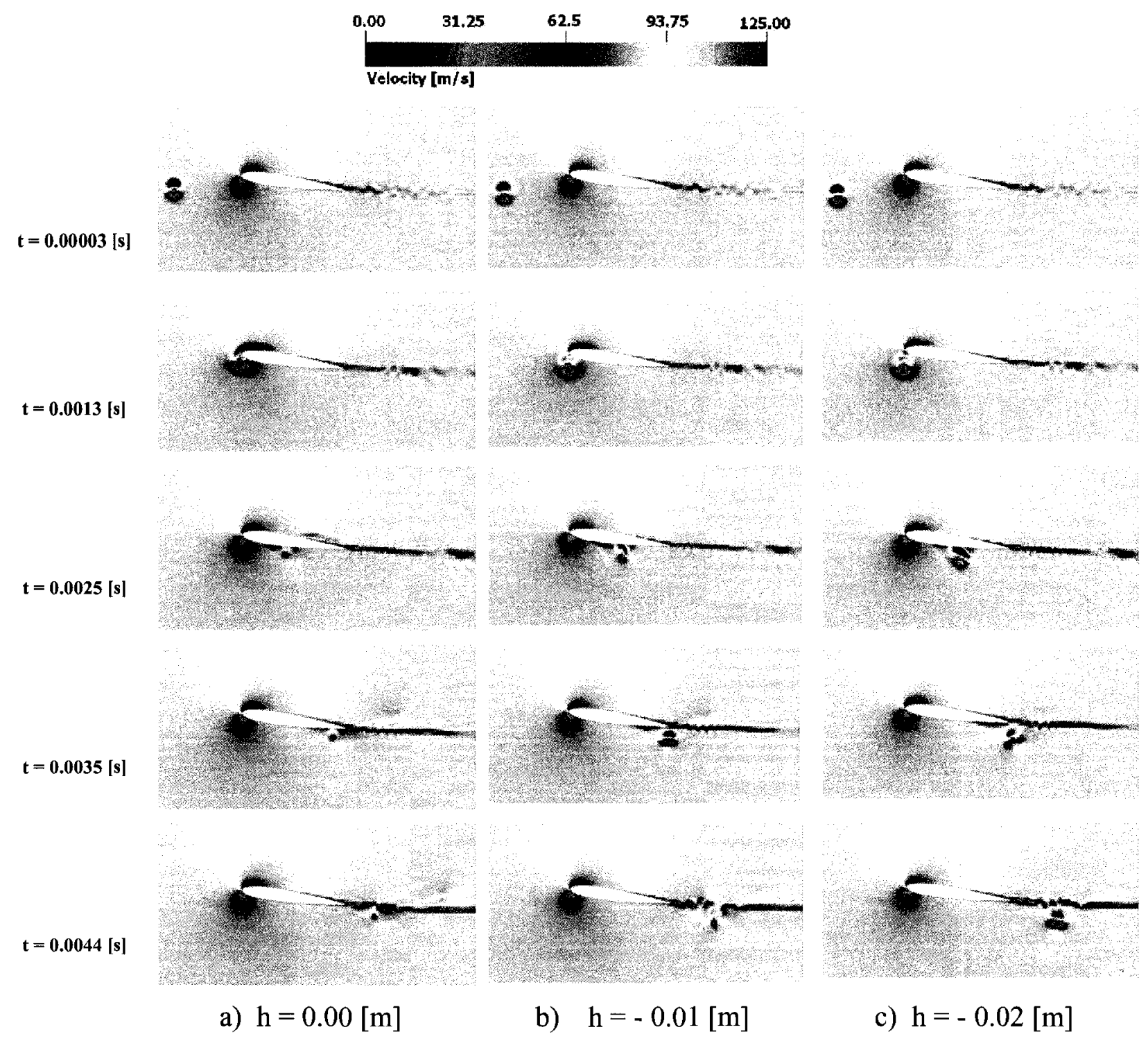

Figure 5.22 Time evolved fluid structures (velocity magnitude), angle of attack $\alpha=10^{\circ}$. 


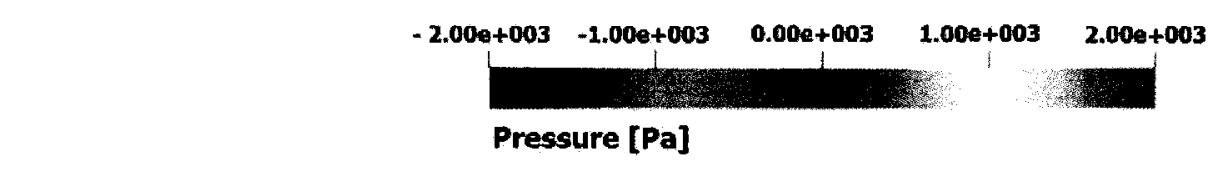

$\mathbf{t}=\mathbf{0 . 0 0 0 0 3}[\mathrm{s}]$
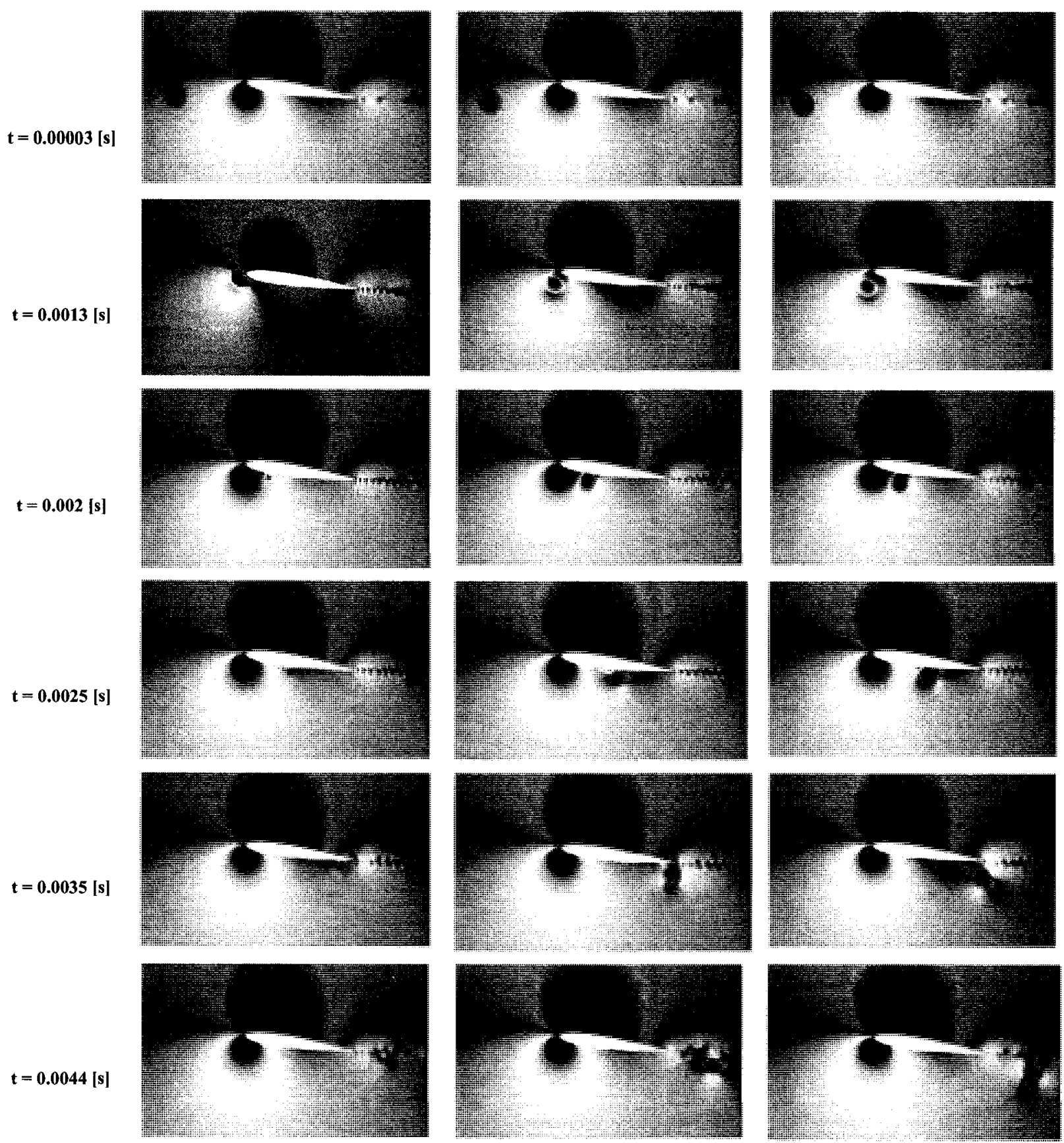

a) $\mathrm{h}=0.00[\mathrm{~m}]$

b) $\mathrm{h}=-0.01[\mathrm{~m}]$

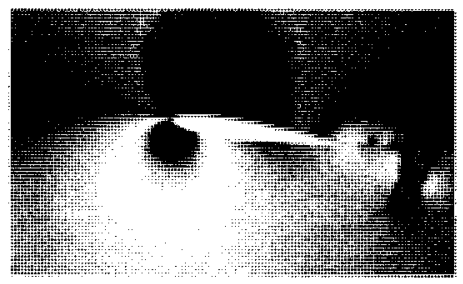

c) $\mathrm{h}=-0.02[\mathrm{~m}]$

Figure 5.23 Time evolved fluid structures (pressure magnitude), angle of attack $\alpha=10^{\circ}$. 
The LES results of the instantaneous magnitude of velocity and pressure for an angle of attack $\alpha=10^{\circ}$ are shown in Figures 5.22 and 5.23, respectively, as a clockwise rotating vortex travels from three different vertical miss-distances, $h=0.00,-0.01$, and $-0.02 \mathrm{~m}$.

The results are very similar to $\alpha=5^{\circ}$, except for the fact that the interaction of the vortex with the trailing edge of the airfoil is stronger. This is reflected into large wake fluctuations when compared against $\alpha=5^{\circ}$.

Figure 5.23 presents the time-varying pressure field for the NACA0012 airfoil, at angle of attack $\alpha=10^{\circ}$ interacting with the oncoming vortex. Due to the airfoil angle of attack, the airfoil-vortex interactions are less severe and as a result of the mechanism of interaction, larger vortex structures can be seen for a vertical miss-distance $\mathrm{h}=-0.01 \mathrm{~m}$, at instant $\mathrm{t}=0.0035 \mathrm{~s}$ and $\mathrm{t}=0.0044 \mathrm{~s}$, when compared with the same corresponding test case for angle of attack $\alpha=5^{\circ}$. Independent of airfoil-vortex vertical miss-distance, large fluctuations of the airfoil wake are observed for an angle of attack $\alpha=10^{\circ}$. These fluctuations are identified in the time-varying aerodynamic coefficients as presented in Figures 5.24 and 5.25. 


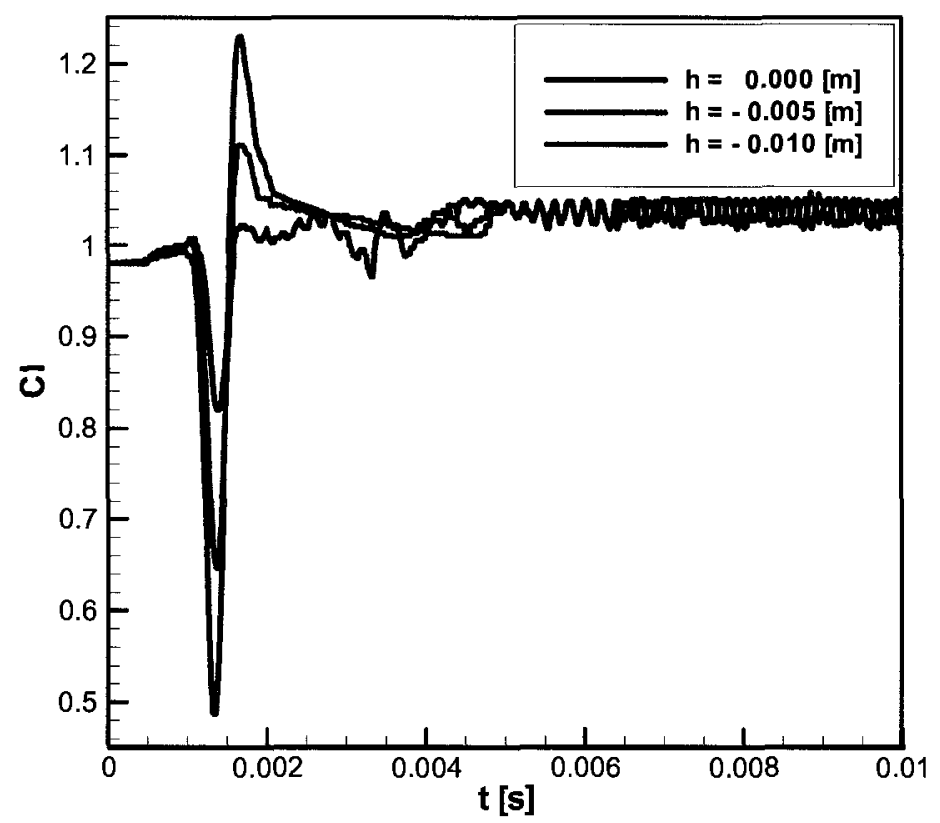

Figure 5.24 Comparison of lift coefficient, angle of attack $\alpha=10^{\circ}$.

From the comparison of lift coefficient for different airfoil-vortex vertical miss-distances, Figure 5.24, it can be seen that as the vertical miss-distance increases, the amplitudes of lift coefficient at the instant when the vortex encounters the airfoil $(t=0.0013 \mathrm{~s})$ decrease. As the airfoil-vortex vertical miss-distance increases, larger fluctuations of lift coefficient are present as the residual component of the vortex travels the chord length of the airfoil, as seen in Figure 5.24, for the vertical miss-distance $h=-0.01 \mathrm{~m}$. As the vortex travels far downstream the airfoil, there is a very similar trend in the lift coefficient behavior for all vertical miss-distances, as seen in Figure 5.24, after the instant $t=0.005 \mathrm{~s}$. 


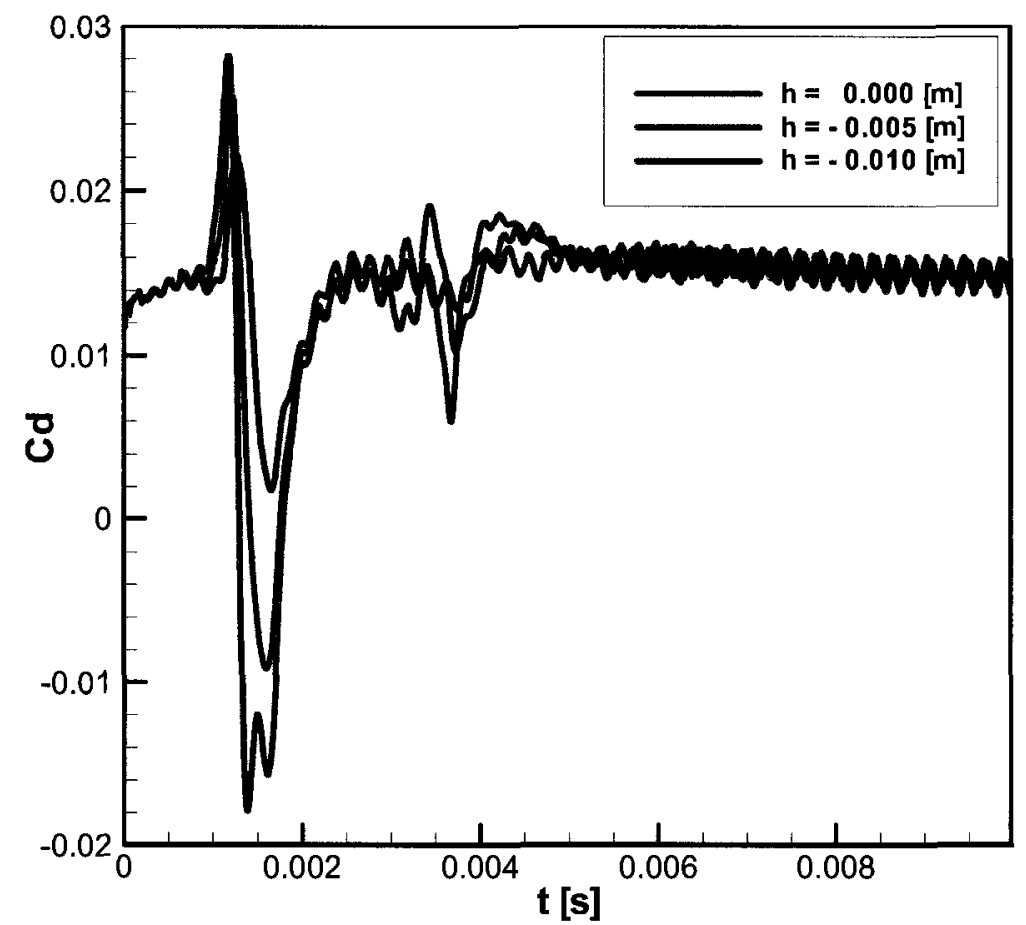

Figure 5.25 Comparison of drag coefficient, angle of attack $\alpha=10^{\circ}$.

For an angle of attack $\alpha=10^{\circ}$, the drag coefficient presents similar behavior with the test cases corresponding to angles of attack $\alpha=0^{\circ}$ and $\alpha=5^{\circ}$ respectively. However, the amount of reverse flow is smaller when compared with the angles of attack $\alpha=0^{\circ}$ and $\alpha=5^{\circ}$, for the same vertical miss-distance. With the increase of the airfoil-vortex vertical miss-distance, the amplitudes of drag coefficient at the instant when the vortex encounters the airfoil decrease, as seen in Figure 5.25, at the instant $t=0.0013 \mathrm{~s}$. Also the amount of reverse flow decreases with the increase of airfoil-vortex vertical miss-distance, and therefore for a vertical missdistance $h=-0.01 \mathrm{~m}$, no reverse flow is observed. However the fluctuations of drag coefficient at the instant when the vortex leaves the airfoil, increase with the increase of vertical miss-distance, as seen in Figure 5.25, at the instant $\mathrm{t}=0.0035 \mathrm{~s}$. 


\subsection{Aerodynamic Comparative Results $\left(0^{\circ}, 5^{\circ}\right.$, and $10^{\circ}$ Angle of Attack)}

Although there are certain similarities in lift coefficient behaviour for the three angles of attack, $\alpha=0^{\circ}, \alpha=5^{\circ}$ and $\alpha=10^{\circ}$ at the instant when the vortex encounters the airfoil, $\mathrm{t}=$ $0.0013 \mathrm{~s}$, there are still significant differences between the three test cases at the instant when the residual component of the vortex leaves the airfoil, $\mathrm{t}=0.0035 \mathrm{~s}$.

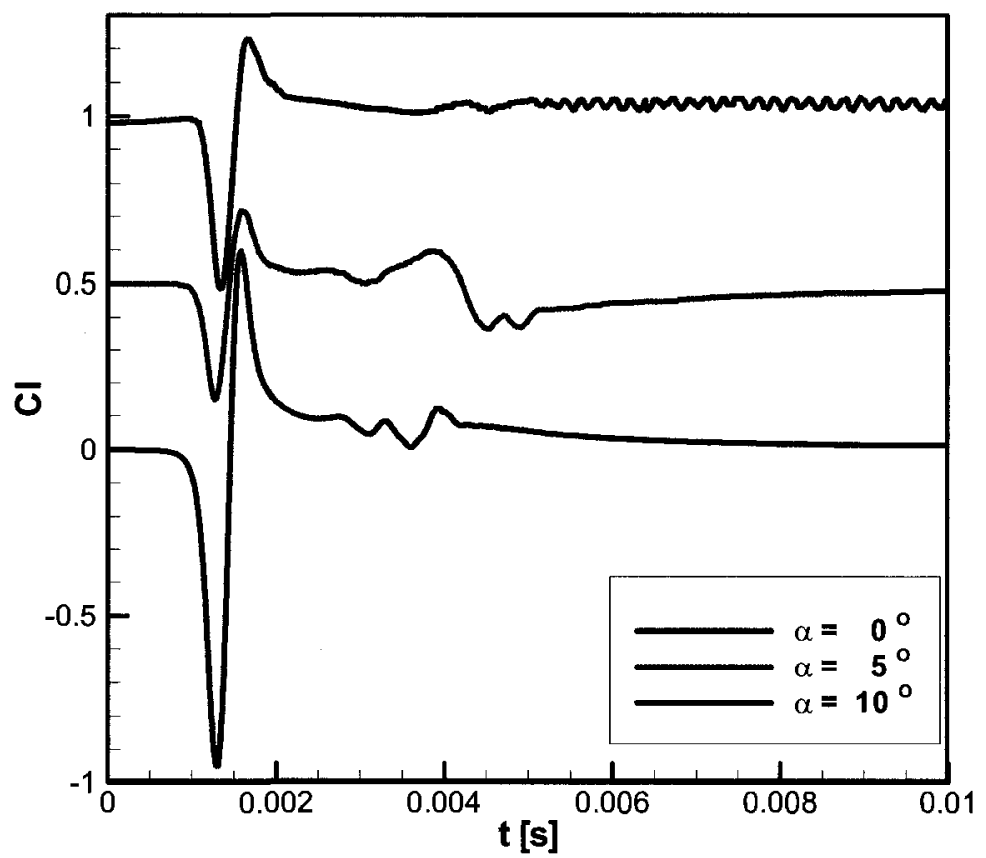

Figure 5.26 Comparison of lift coefficient, $h=0.00 \mathrm{~m}$. 


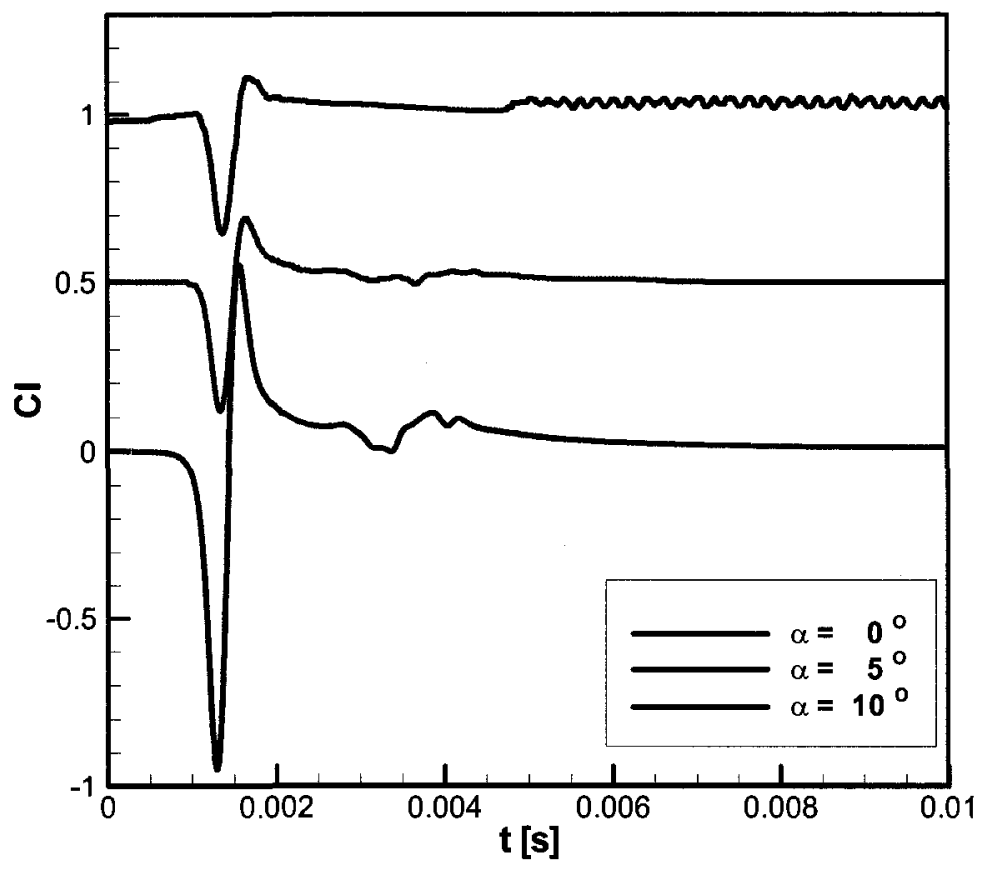

Figure 5.27 Comparison of lift coefficient, $h=-\mathbf{0 . 0 0 5 m}$.

A comparison of lift coefficient corresponding to the three angles of attack, for a vertical miss-distance $h=0.00 \mathrm{~m}$, is presented in Figure 5.26. As expected, higher angles of attack give higher values of lift. Although the trends in lift coefficient are very similar, the magnitude of the variation of the lift coefficient decreases when the angle of attack is increased. Increasing the airfoil-vortex vertical miss-distance to $h=-0.005 \mathrm{~m}$, the lift coefficient presents a similar trend, as seen in Figure 5.27. Notice the distinctive oscillatory behaviour (fluctuations) for $\alpha=10^{\circ}$ in both figures. 


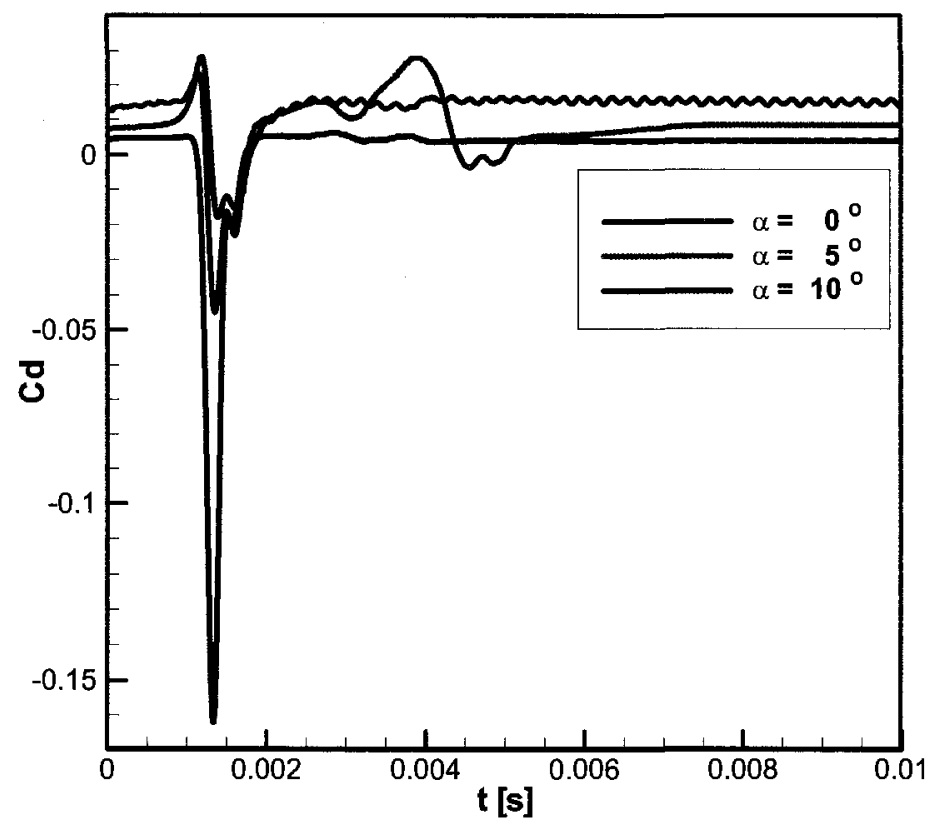

Figure 5.28 Comparison of drag coefficient, $h=0.00 \mathrm{~m}$.

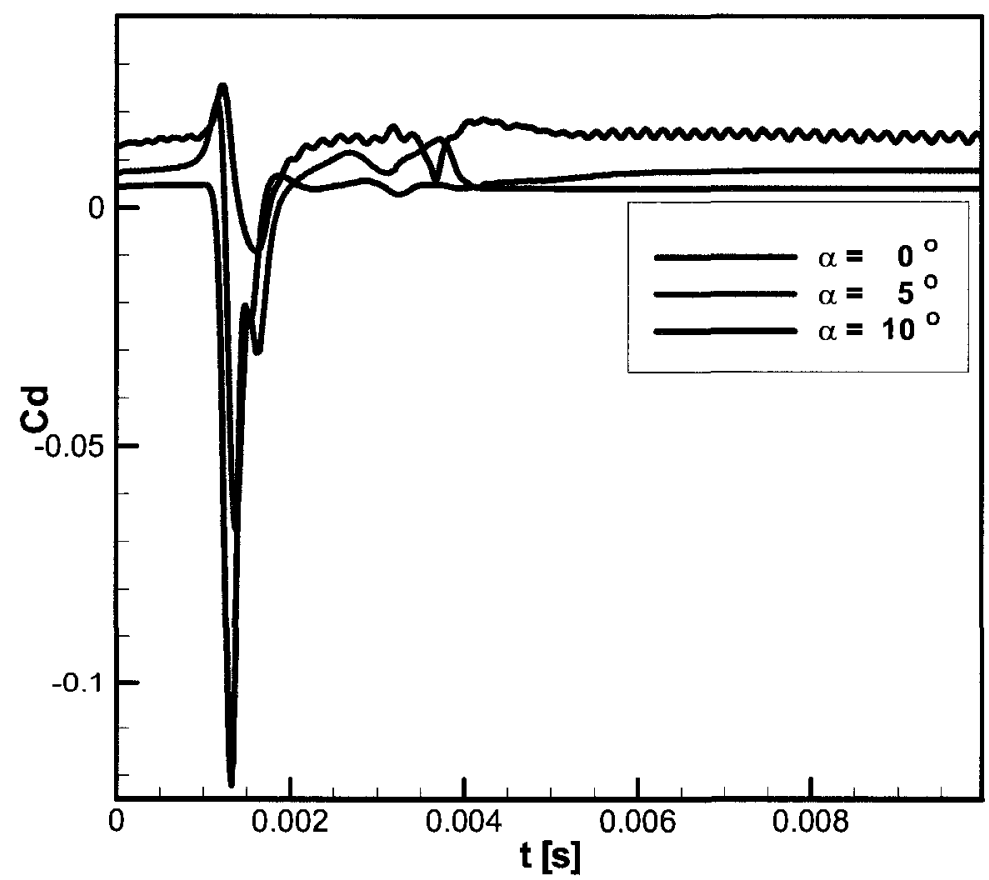

Figure 5.29 Comparison of drag coefficient, $h=-\mathbf{0 . 0 0 5 m}$. 
Figures 5.28 and 5.29 shows the drag coefficient for two different vertical miss-distances, $\mathrm{h}=$ 0.00 and -0.005 , respectively, both at three different airfoil angles of attack $\alpha=0^{\circ}, \alpha=5^{\circ}$, and $\alpha=10^{\circ}$. As mentioned before, an increase of drag coefficient is followed by a sudden decrease for angles of attack $\alpha=5^{\circ}$ and $\alpha=10^{\circ}$, as the vortex approaches the airfoil. This behaviour is different from $\alpha=0^{\circ}$ (starting with a sudden decrease). For all three angles of attack $\alpha=0^{\circ}, \alpha=5^{\circ}$, and $\alpha=10^{\circ}$, as the residual component of the vortex travels far downstream the airfoil, it merges into the wake and dissipates, causing the drag coefficient to converge to a constant value, which corresponds to the case of uniform flow past NACA0012 at different angles of attack, for Reynolds number $R e=1.3 \times 10^{6}$. The fluctuations (mentioned before) in the drag coefficients are present for $\alpha=10^{\circ}$.

\subsection{Aerodynamic Results (Vortex Core Size)}

Besides the influence of airfoil-vortex vertical miss-distance and angle of attack, the present analysis investigates the influence of vortex characteristics (core size) on the airfoil-vortex mechanism of interaction. The computations are carried out for three different vortex core

sizes $r=0.3 c, r=0.4 c, r=0.5 c$, where $c$ is the chord of the airfoil. In this case the strength of the vortex is kept constant ( $25 \%$ higher than free-stream velocity) while the vortex core size varies. 


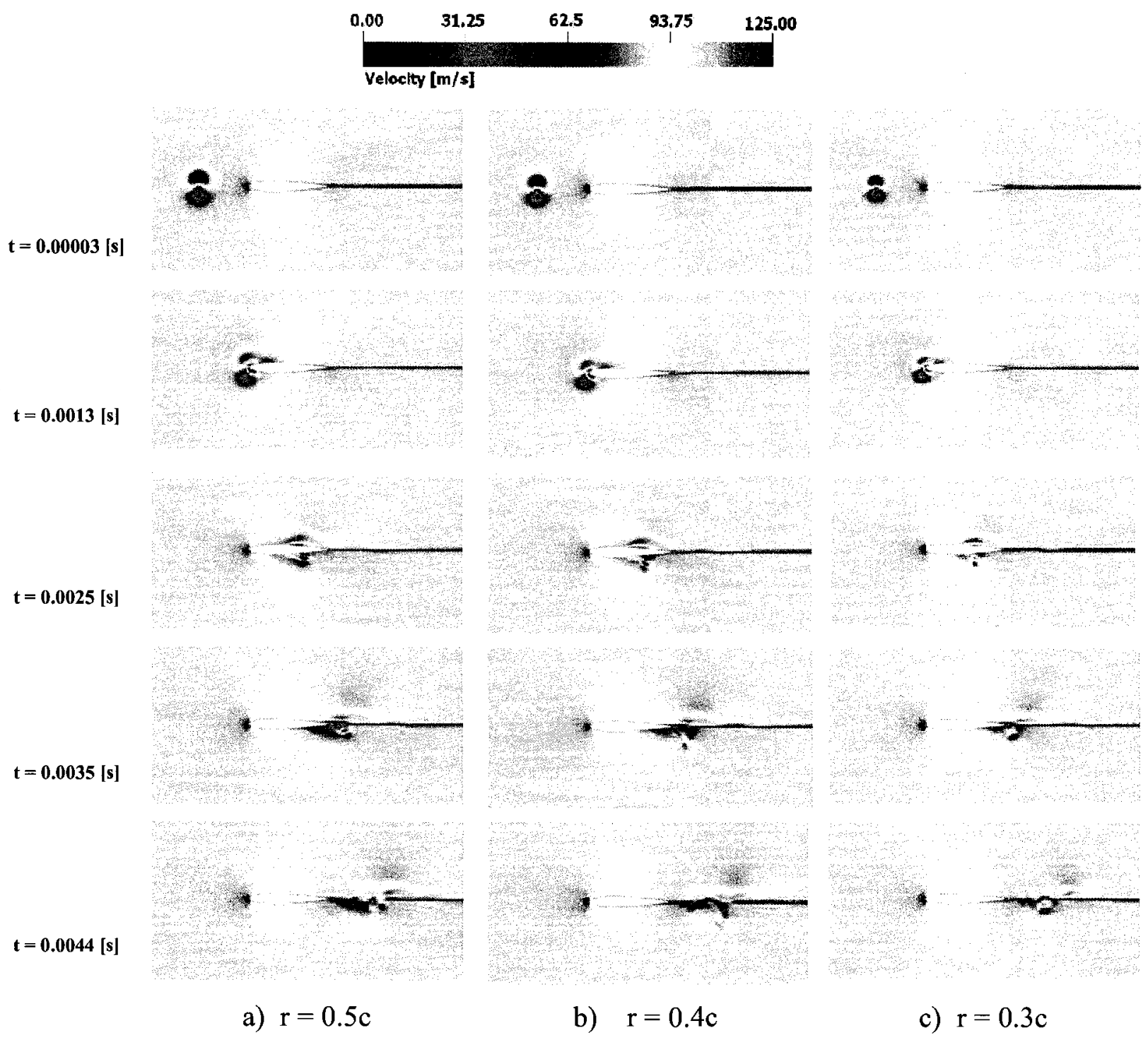

Figure 5.30 Time evolved fluid structures (velocity magnitude).

Figure 5.30 shows the instantaneous magnitude of velocities, using LES, for an angle of attack $\alpha=0^{0}$, at five different instants in time, as a clockwise rotating vortex travels at the same vertical miss-distance, $\mathrm{h}=0.00 \mathrm{~m}$. Due to the airfoil-vortex mechanism of interaction, the vortex splits into two residual components of different strengths and different sizes, traveling on two separate paths, one below and the other above the airfoil, as seen at instant $t$ $=0.0025 \mathrm{~s}$. For all test cases, the residual component of the vortex traveling underneath of the 
airfoil has a lower magnitude than the one traveling above the airfoil. From the velocity field it can be seen that the strength and size of the vortex residual components decrease with the decrease of vortex core size.

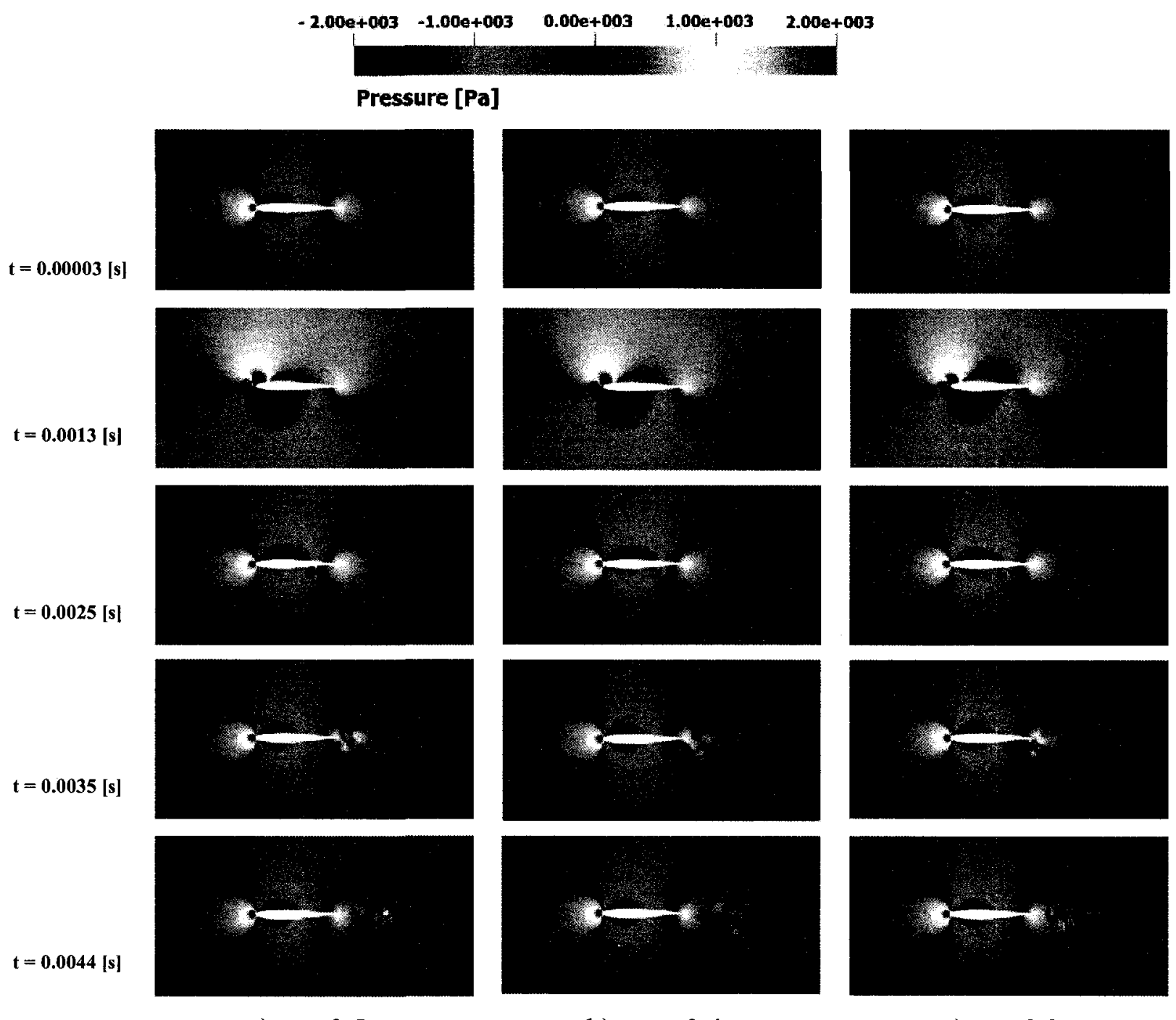
a) $\mathrm{r}=0.5 \mathrm{c}$
b) $\mathrm{r}=0.4 \mathrm{c}$
c) $\mathrm{r}=0.3 \mathrm{c}$

Figure 5.31 Time evolved fluid structures (pressure magnitude). 
The change in velocity field distribution influences the pressure distribution around the airfoil and causes fluctuations of the stagnation point along the upper and lower surface of the airfoil, as seen in Figure 5.31. This movement of the stagnation point affects the lift on the blade. The rate of change of lift is related to the pressure propagated to an observer. The unsteady vortex motion induces pressure fluctuations in the proximity of the blade, and results in a series of expansion/compression waves, which propagate upstream. The change in pressure at the instant when the vortex encounters the airfoil is associated with the BVI noise.

The airfoil-vortex mechanism of interaction has impact on both velocity and pressure field and as anticipated, it influences the aeroacoustic field as well. The influence of airfoilvortex mechanism of interaction is also reflected into the aerodynamic coefficients.

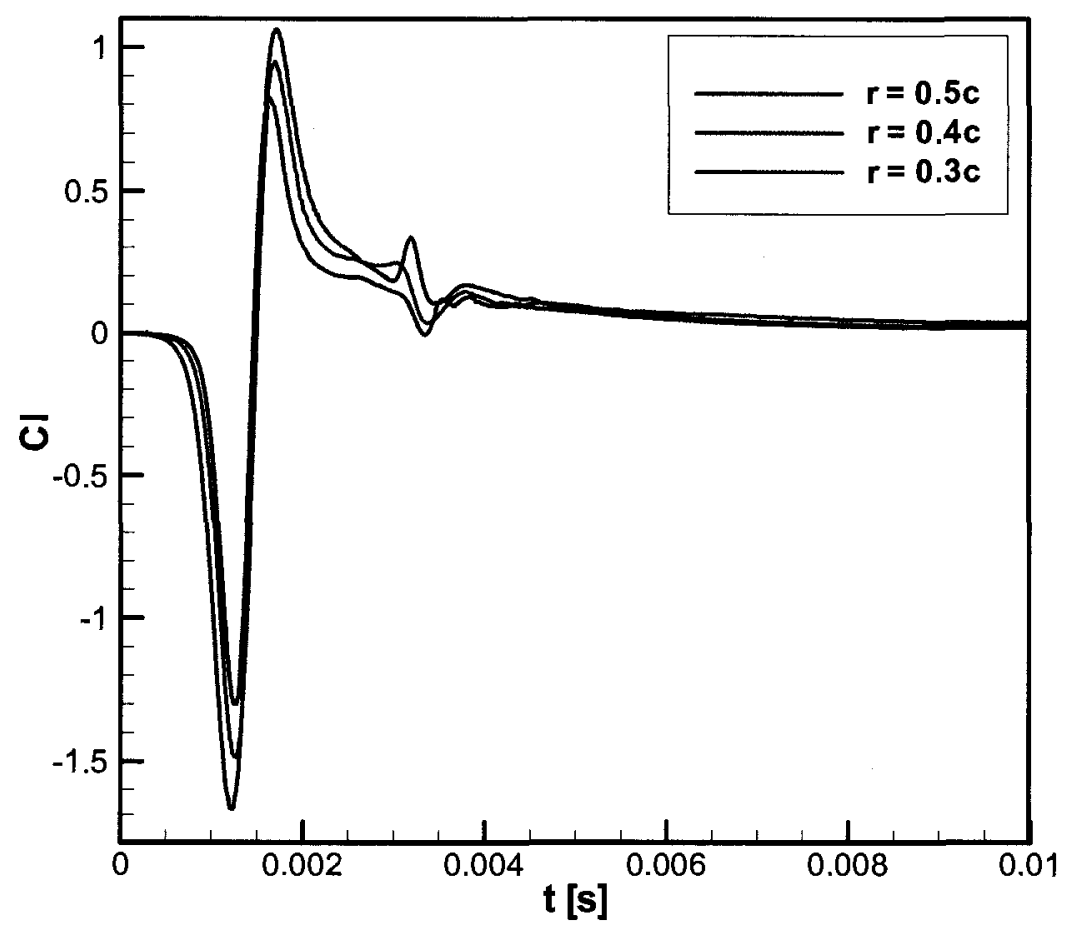

Figure 5.32 Comparison of lift coefficient. 
Figure 5.32 shows a comparison of lift coefficient for three different test cases, based on the vortex core sizes, $r=0.5 \mathrm{c}, 0.4 \mathrm{c}$, and $0.3 \mathrm{c}$. For all test cases, the lift coefficient presents a sudden jump at the instant when the vortex encounters the airfoil $(t=0.0013 \mathrm{~s})$. The amplitude of the lift jump decreases as the vortex core size decreases. This decrease is due to the fact that the airfoil-vortex interactions are less severe and have less impact on the flow field. Notice that for all test cases, there are fluctuations in lift coefficient as the residual component of the vortex leaves the airfoil and merges into the airfoil wake, $t=0.0032 \mathrm{~s}$. The fluctuations of lift coefficient at the instant when the residual component of the vortex leaves the airfoil decrease with the decrease of vortex core size. As the residual component of the vortex travels away from the airfoil, the lift coefficient converges asymptotically to zero, from positive values, for all test cases.

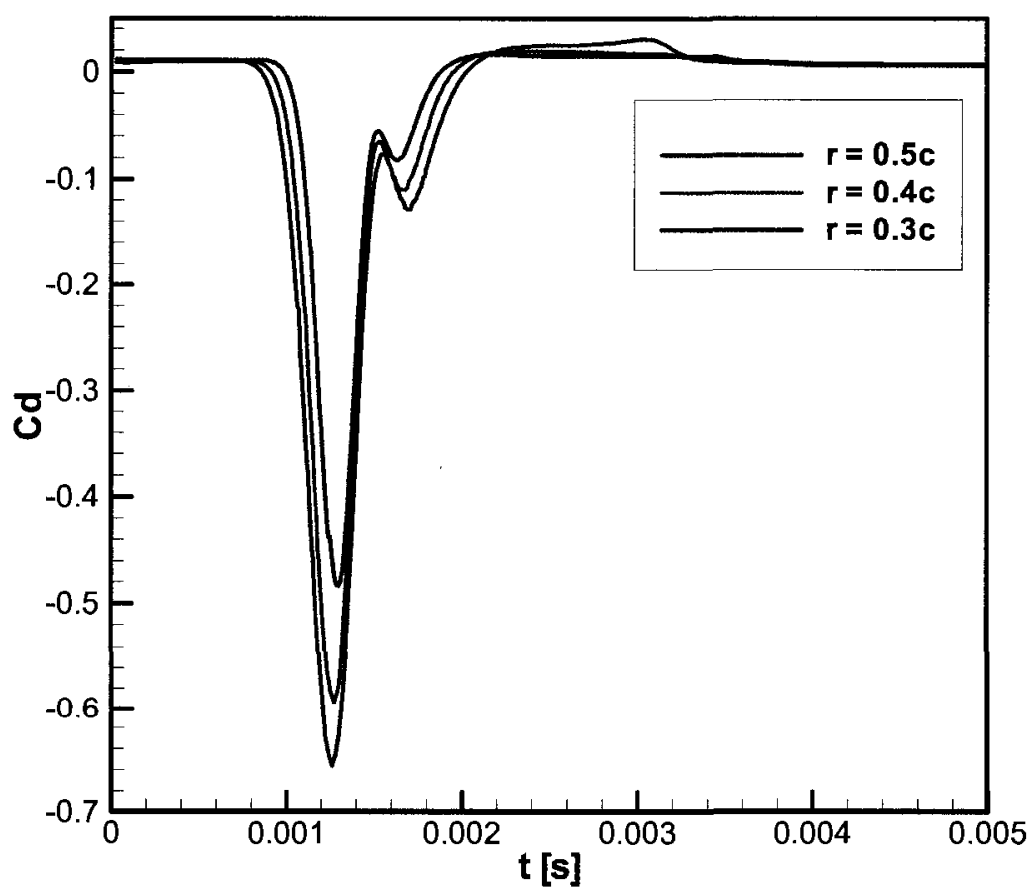

Figure 5.33 Comparison of drag coefficient. 
In Figure 5.33, the comparison of time-varying drag coefficient for three different test cases, based on the vortex core size, $r=0.5 \mathrm{c}, 0.4 \mathrm{c}$, and $0.3 \mathrm{c}$, is shown. For all test cases, large negative values of drag coefficient are observed at the instant when the vortex encounters the airfoil, $\mathrm{t}=0.0013 \mathrm{~s}$. These negative values are caused primarily by separation and reverse flow over the surface of the airfoil. It can be seen that the largest amount of reverse flow occurs when the vortex core size is the largest, $r=0.5 \mathrm{c}$. As the vortex core size decreases, the airfoil-vortex interactions are less and less severe and likewise the separation, resulting in a decrease in magnitude of the negative peaks of drag coefficient associated with reverse flow. A small change in the vortex core size has a large impact on the drag coefficient at the instant when the vortex encounters the airfoil. Due to the airfoil-vortex mechanism of interaction, the distorted vortex causes fluctuations of drag coefficient as it travels the chord length of airfoil. From the drag coefficient comparison, Figure 5.33, it can be seen that for the case of a vortex core size $r=0.5 \mathrm{c}$, the drag coefficient presents a slightly higher value at the instant when the vortex leaves the airfoil and this is due to the vortex core size and implicitly to the airfoilvortex mechanism of interaction.

\subsection{Aeroacoustic Results}

The airfoil-vortex mechanism of interaction not only influences the aerodynamic coefficients, but also the aeroacoustic field. An aeroacoustic investigation is conducted in order to examine the influence of spanning angle $\beta$ (measured with respect to the rotor disc plane), airfoil-vortex vertical miss-distance, airfoil angle of attack, and vortex core size on the Sound Pressure Level (SPL), which can be obtained from the history of the pressure field as 
described at the end of Chapter 4. The following aeroacoustic investigation is based on a numerical approach using large-eddy simulation. The change in velocity distribution field influences the pressure distribution around the airfoil and causes fluctuations of the stagnation point along the upper and lower surface of the airfoil.

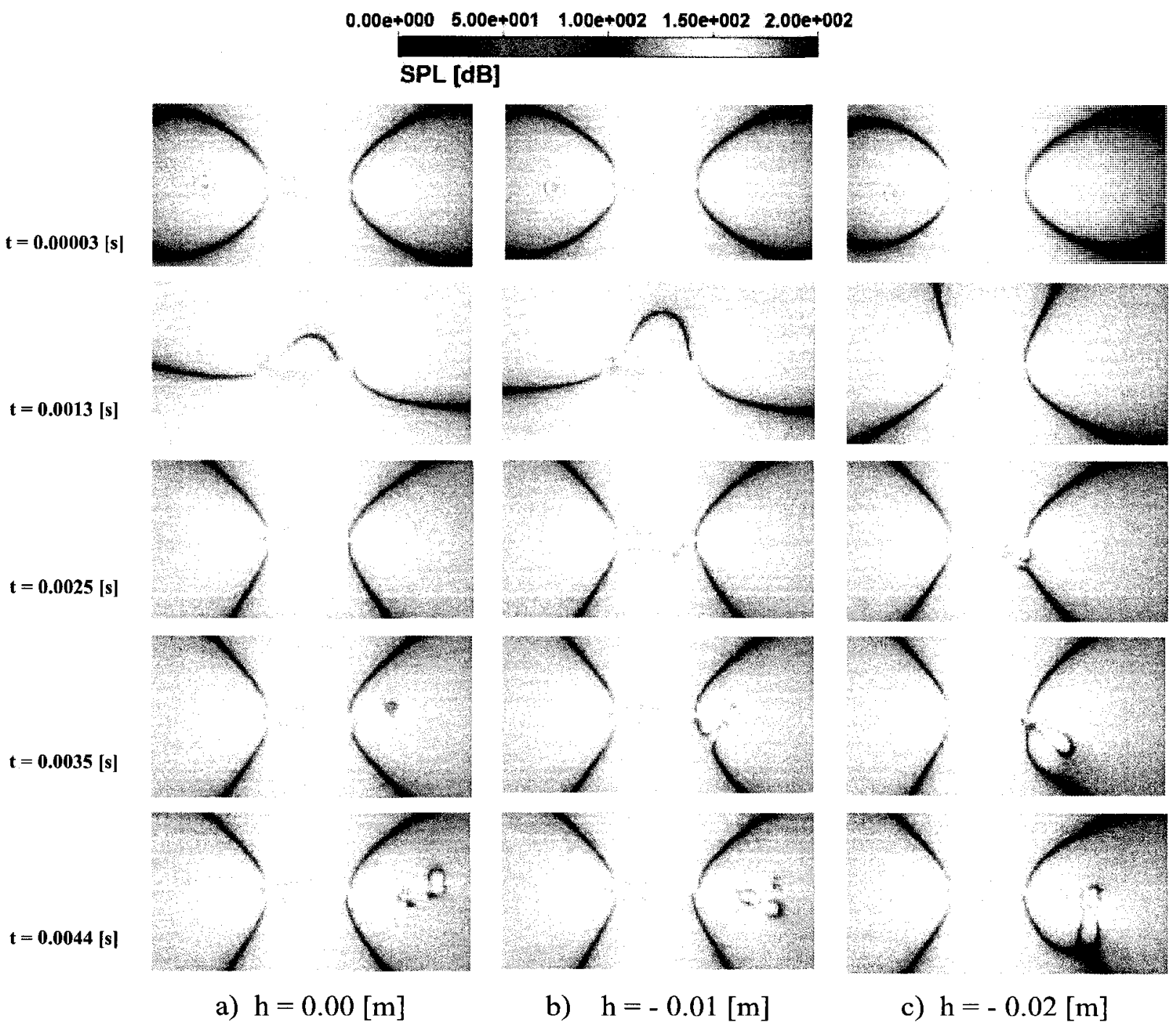

Figure 5.34 Time history of SPL, for angle of attack $\alpha=0^{\circ}$.

Figure 5.34 presents the time-varying sound pressure level (SPL) at different instants in time for three different airfoil-vortex miss-distances and for a NACA0012 airfoil at angle of attack 
$\alpha=0^{0}$. For all test cases, contour lines of constant magnitude (100dB) can be observed. In the figure, red and yellow regions represent noisier levels than green (100dB), while light and dark blue are less noisy than green. For all test cases and at the instant $t=0.00003 \mathrm{~s}$, the contour lines describe a hyperbola with the foci located on the camber line of the airfoil. Also, for all test cases, the contour lines exhibit a transient behaviour in time, being dependent of airfoil-vortex vertical miss-distance. The transient behaviour of the contour lines is described in the following. For all test cases, as the vortex approaches the airfoil, the upper branches of hyperbola (above camber line) merge together and generate a convex bell shape contour, whose size decreases as the airfoil-vortex interaction progresses. The bell shape contour reaches a minimum at the instant $t=0.0013 \mathrm{~s}$. This means that an observer stationed at the front and lower part of the airfoil would experience a steep change in SPL. As the vortex passes the leading edge, there is a sudden change in the bell shape contour, this becoming a concave bell shape, as a result of merging process between the lower branches of hyperbola. When the residual component of the distorted vortex travels the first half of the airfoil chord, the constant SPL fronts redefine a hyperbolic shape, as seen at the instant $t=$ $0.0025 \mathrm{~s}$.

As the vortex leaves the airfoil, the SPL contour lines define a hyperbolic profile for all test cases. Although the contour profile exhibits very similar pattern for all test cases, there are still differences in the magnitude of the bell shape contours and this can be seen best at instant $t=0.0013 \mathrm{~s}$. It can be seen that the size of the bell shape contour increases as the airfoil-vortex vertical miss-distance increases, and this is a result of the influence of airfoilvortex vertical miss-distance on the mechanism of interaction, since the mechanism of interaction affects the airfoil surrounding flow field. 
Also, it can be seen that the residual component of the vortex distorts the lower branch of the hyperbola in a different manner, for each test case, at the instant when it leaves the airfoil, $t=0.0035 \mathrm{~s}$. This result is also associated with the airfoil-vortex mechanism of interaction since the characteristics of the residual component of the vortex are a function of airfoil-vortex mechanism of interaction. From Figure 5.34, it can be seen that the residual component of the vortex has a larger impact on the constant SPL contour lines when the airfoil-vortex vertical miss-distance is large. This is a result of the fact that increasing the vertical miss-distance, the airfoil-vortex interaction is less severe and, hence, the vortex preserves better its characteristics, having a stronger effect on the surrounding flow field. As the residual component of the vortex travels far downstream, the constant SPL contour lines describe a hyperbolic profile of constant magnitude (100dB), for all test cases. 


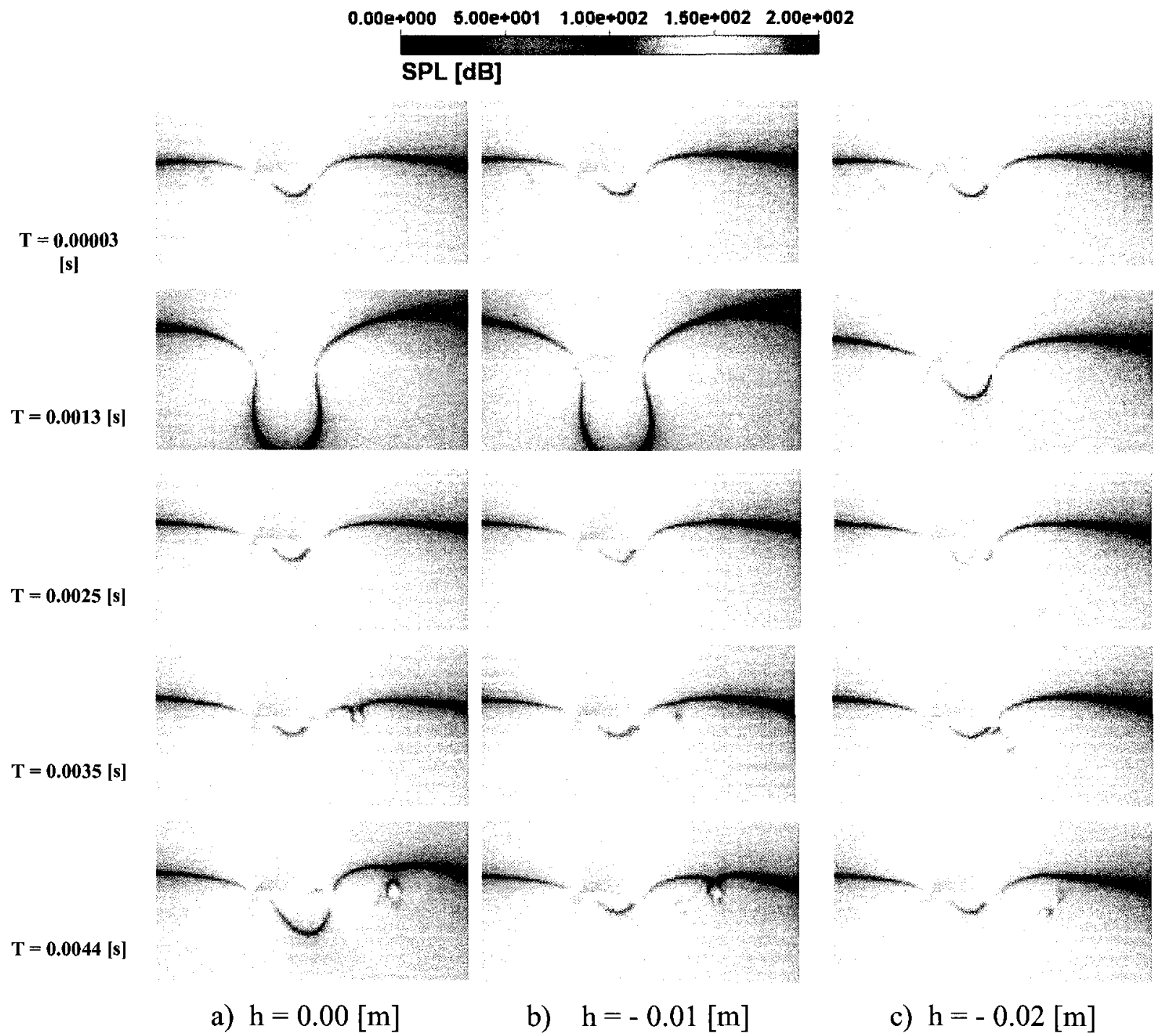

Figure 5.35 Time history of SPL, for angle of attack $\alpha=5^{\circ}$.

Similar to angle of attack $\alpha=0^{0}$ an aeroacoustic investigation is conducted based on the Sound Pressure Level (SPL), for an angle of attack $\alpha=5^{0}$. In this sense, Figure 5.35 presents the time-varying sound pressure level (SPL) at different instants in time, for three different airfoil-vortex vertical miss-distances, and for an angle of attack $\alpha=5^{\circ}$. From Figure 5.35, it can be seen that the angle of attack influences the airfoil-vortex mechanism of interaction and 
implicitly the time-varying SPL values. At similar instants in time, there are significant differences in the trend of time-varying SPL values, for the angles of attack $\alpha=0^{\circ}$ and $\alpha=$ $5^{\circ}$, as seen in Figure 5.34 and Figure 5.35, respectively. Whereas for the angle of attack $\alpha=$ $0^{\circ}$ the SPL contours of constant value define a hyperbolic profile, for the angle of attack $\alpha=$ $5^{\circ}$ the SPL contours of constant value define a parabolic profile at all instants in time. This parabolic trend is a result of the influence of angle of attack on the airfoil-vortex mechanism of interaction. Besides the differences between the SPL for the two angles of attack, there are other differences among the test cases analyzed for the angle of attack $\alpha=5^{\circ}$ based on the airfoil-vortex vertical miss-distance, as seen in Figure 5.35.

Large differences due to the airfoil-vortex vertical miss-distance can be seen at the instant when the vortex encounters the airfoil, $t=0.0013 \mathrm{~s}$. The SPL parabolic profile presents a minimum for a vertical miss-distance $h=-0.02 \mathrm{~m}$, at instant $\mathrm{t}=0.0013 \mathrm{~s}$. As the vortex passes the leading edge, a similar trend and magnitude of SPL values is observed, as seen at instants $t=0.0025$ and $0.0035 \mathrm{~s}$. It is worth to notice the presence of large differences among the three test cases at the instant when the residual component of the vortex travels downstream the airfoil, as seen at instant $\mathrm{t}=0.0044 \mathrm{~s}$ in Figure 5.35. The residual component of the vortex alters the contour line of constant SPL $(100 \mathrm{~dB})$ in a different manner, and this is also a result of the airfoil-vortex mechanism of interaction due to the vertical miss-distance.

The airfoil-vortex mechanism of interaction, as a result of change of angle of attack, induces large discontinuities of the constant SPL contour lines at the instants when the residual component of the vortex leaves the airfoil and travels downstream, for example, at $t$ $=0.0035$ and $0.0044 \mathrm{~s}$. These discontinuities of SPL contour lines can be best observed at the 
trailing edge region. These discontinuities are also observed for an angle of attack $\alpha=0^{\circ}$; however they are smaller compared with the ones corresponding to angle of attack $\alpha=5^{\circ}$.

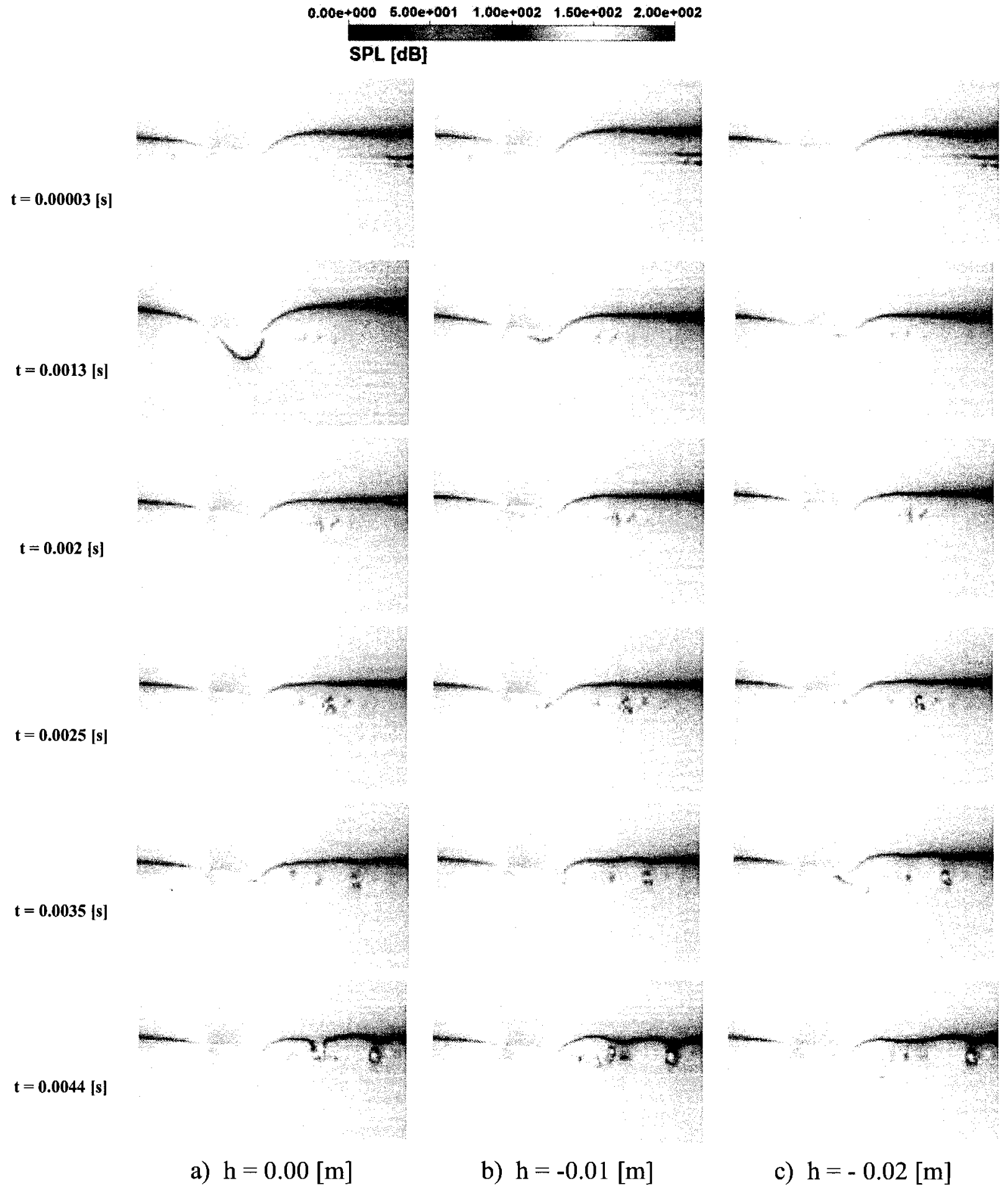

Figure 5.36 Time history of SPL, for angle of attack $\alpha=10^{\circ}$. 
Figure 5.36 presents the time-varying SPL, for an angle of attack $\alpha=10^{\circ}$, for three different vertical miss-distances. Similar to test cases for angles of attack $\alpha=0^{\circ}$ and $\alpha=5^{\circ}$, SPL fronts of constant value can be observed for an angle of attack $\alpha=10^{\circ}$. However, for an angle of attack $\alpha=10^{\circ}$ the front lines tend to align with the airfoil camber. With the increase of airfoil-vortex vertical miss-distance, the influence of the vortex on the SPL contour lines diminishes.

Already knowing the general trend of the time-varying SPL, it is of interest to quantify the acoustic noise due to the airfoil-vortex interaction. In order to pursue this analysis, the influence of the spanning angle (measured with respect to the rotor plane) on the aeroacoustic field is investigated, as schematically presented in Figure 5.37. The spanning angle is investigated for a range of $5^{\circ}$ to $45^{\circ}$ and an incrementing angle of $5^{\circ}$. The SPL fluctuations are monitored at points along the paths described by the incrementing spanning angles. These interrogation points can be assumed as being microphones (in a wind tunnel experiment). In this sense horizontal points, equidistantly placed in a Cartesian coordinate system originating at the airfoil leading edge are subject of investigations. The vertical coordinates of the interrogation points are given by the equation (5.4).

$$
y=x \tan \beta
$$

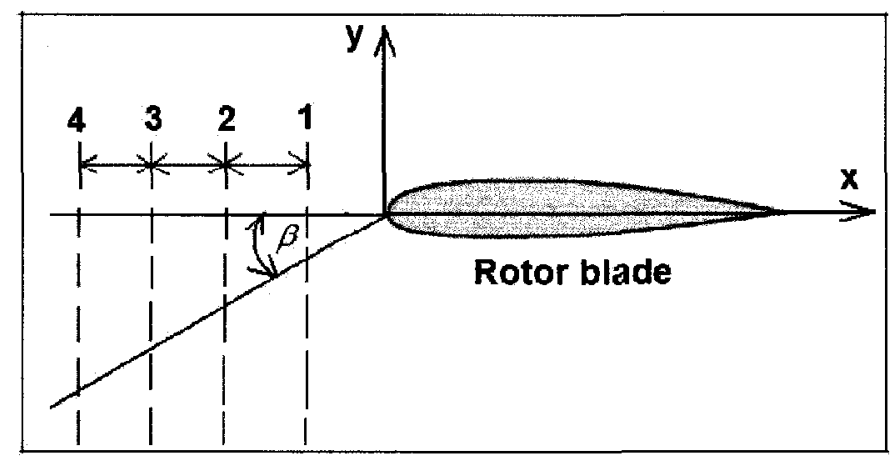

Figure 5.37 Schematic view of aeroacoustics interrogation paths. 
Although the frequency-domain representation of SPL is a common practice in the aeroacoustic studies, in the present analysis, for clarity reasons (due to the small time-step), a time-domain representation is preferred.

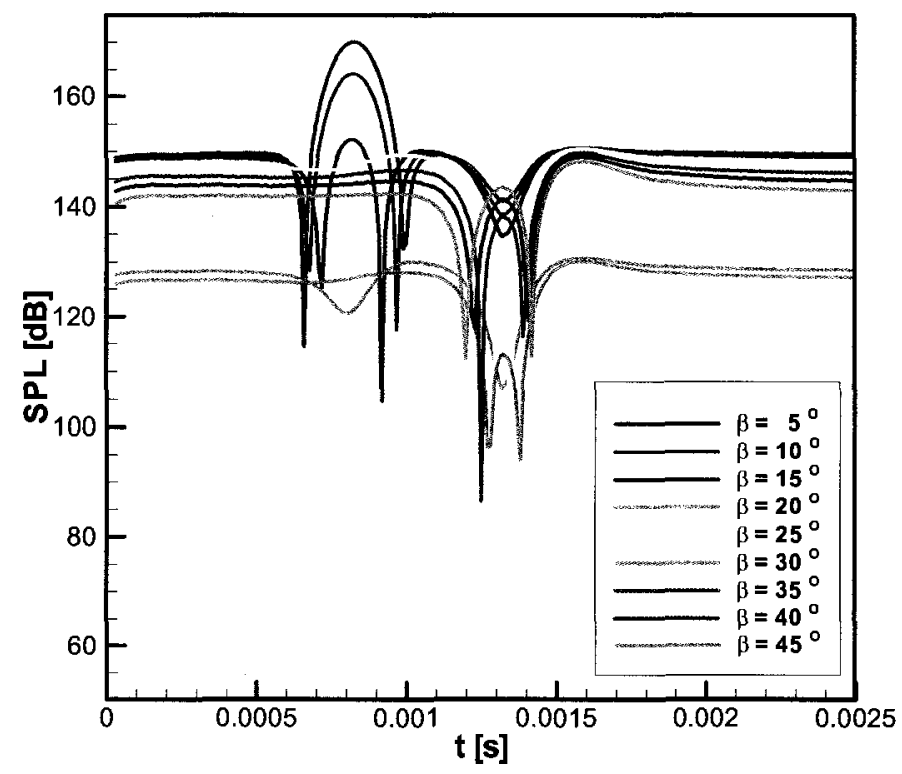

Figure 5.38 Comparison of SPL, $\alpha=0^{0}, \mathrm{~h}=-\mathbf{0 . 0 0} \mathrm{m}, \mathrm{x}=-\mathbf{0 . 0 5 m}$.

Figure 5.38 presents the time variation of the Sound Pressure Level (SPL) at the fixed horizontal location $\mathrm{x}=-0.05 \mathrm{~m}$ for nine different values of the spanning angle, $\beta$, and for the airfoil-vortex vertical miss-distance, $\mathrm{h}=0.00 \mathrm{~m}$. Due to the fact that after the instant $\mathrm{t}=$ $0.0025 \mathrm{~s}$, there is no variation in the SPL trend, reaching a constant value, and for clarity reasons, only a quarter of data set values are presented.

The SPL presents impulsive "spikes" at the instant when the vortex encounters the airfoil $(\mathrm{t}=0.0013 \mathrm{~s})$. It is important to notice that the location of the interrogation point is obviously very important in the aeroacoustic analysis. The points located near the traveling path of the vortex sense not only the AVI noise but also the acoustic noise generated by the 
vortex itself. The vortex disturbs the flow field neighboured to its path and this is felt at the interrogation points. The vortex-generated acoustic noise is identified by the first large SPL peaks in the SPL time history. It is obvious that the vortex-generated acoustic noise is identified at different instants in time, depending on the location of interrogation points. Hence, the points located far from the leading edge (close to the vortex releasing point) are the first ones sensing the vortex-generated acoustic noise. Consequently, the interrogation points located close to the leading edge are the ones that sense the vortex-generated acoustic noise at a later instant in time. It is important to mention that the interrogation points located in a horizontal distance less than the diameter of the vortex core sense the combined effect of both, vortex-generated acoustic noise and AVI noise.

If the phase difference of the vortex-generated acoustic noise is defined by the horizontal location, the magnitude of the vortex-generated acoustic noise is defined by the vertical location of the interrogation points. Hence, the points located far from the vortex traveling path, exhibit lower magnitudes of SPL associated with the vortex-generated acoustic noise, while the ones close to the vortex traveling path present higher magnitude values. This can be seen in Figure 5.38, for a horizontal distance from the leading edge $\mathrm{x}=$ $0.05 \mathrm{~m}$.

From Figure 5.38, it can be seen that all the interrogation points sense the BVI noise, at about the same instant $t=0.0013 \mathrm{~s}$. However, the magnitude of the SPL decreases with the increase of the spanning angle $\beta$. The first large peaks of SPL seen in Figure 5.38 are associated with the vortex-generated acoustic noise. These SPL peaks occur at the instant when the vortex approaches the interrogation points which are close to its traveling path. As it was previously stated, the magnitude of these peaks decreases with the increase of the 
vertical distance, measured from the vortex traveling path, or increase of the spanning angle $\beta$. This is a result of the fact that the interrogation point senses less and less the influence of the vortex on the flow field.

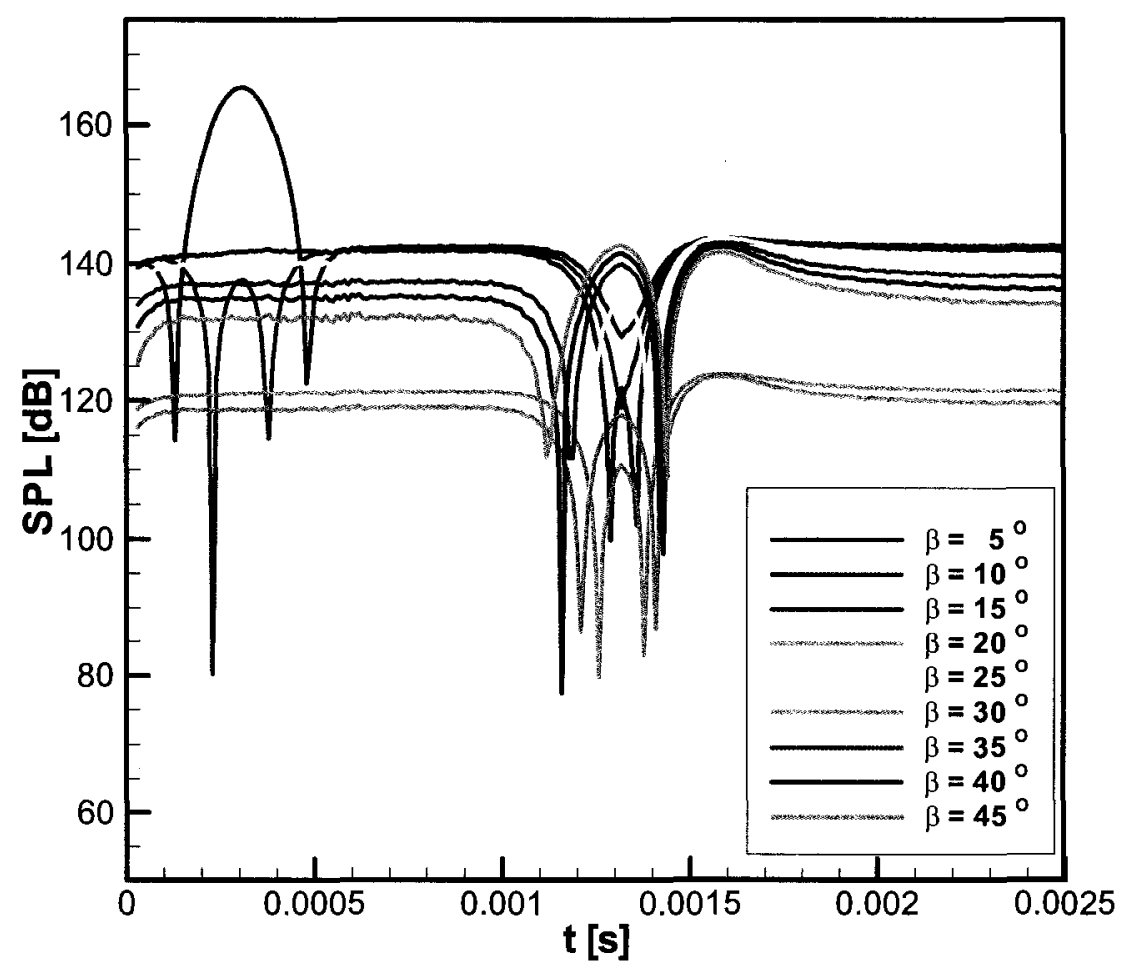

Figure 5.39 Comparison of SPL, $\alpha=0^{0}, \mathrm{~h}=-0.00 \mathrm{~m}, \mathrm{x}=0.1 \mathrm{~m}$.

Figure 5.39 presents the time-varying SPL for a distance from airfoil leading edge $\mathrm{x}=-0.1 \mathrm{~m}$. From Figure 5.39 it can be seen that increasing the horizontal distance from the leading edge, the vortex-generated acoustic noise is sensed at an earlier instant in time since the interrogation point is closer to the vortex releasing location. Also it can be seen that a small change in horizontal distance from the leading edge, has a large impact on the SPL behaviour since the vertical distance increases substantially, function of the spanning angle. 
From Figure 5.39 it can be seen that in this case only the spanning angle $\beta=5^{0}$ senses the vortex-generated acoustic noise, the other spanning angles define points which are located far away from the vortex traveling path and hence sensing less and less the effect of the vortex on the flow field. The spanning angle $\beta=10^{\circ}$ defines a point that still senses the vortex-generated acoustic noise, but much less than a point defined by the spanning angle $\beta=5^{0}$.

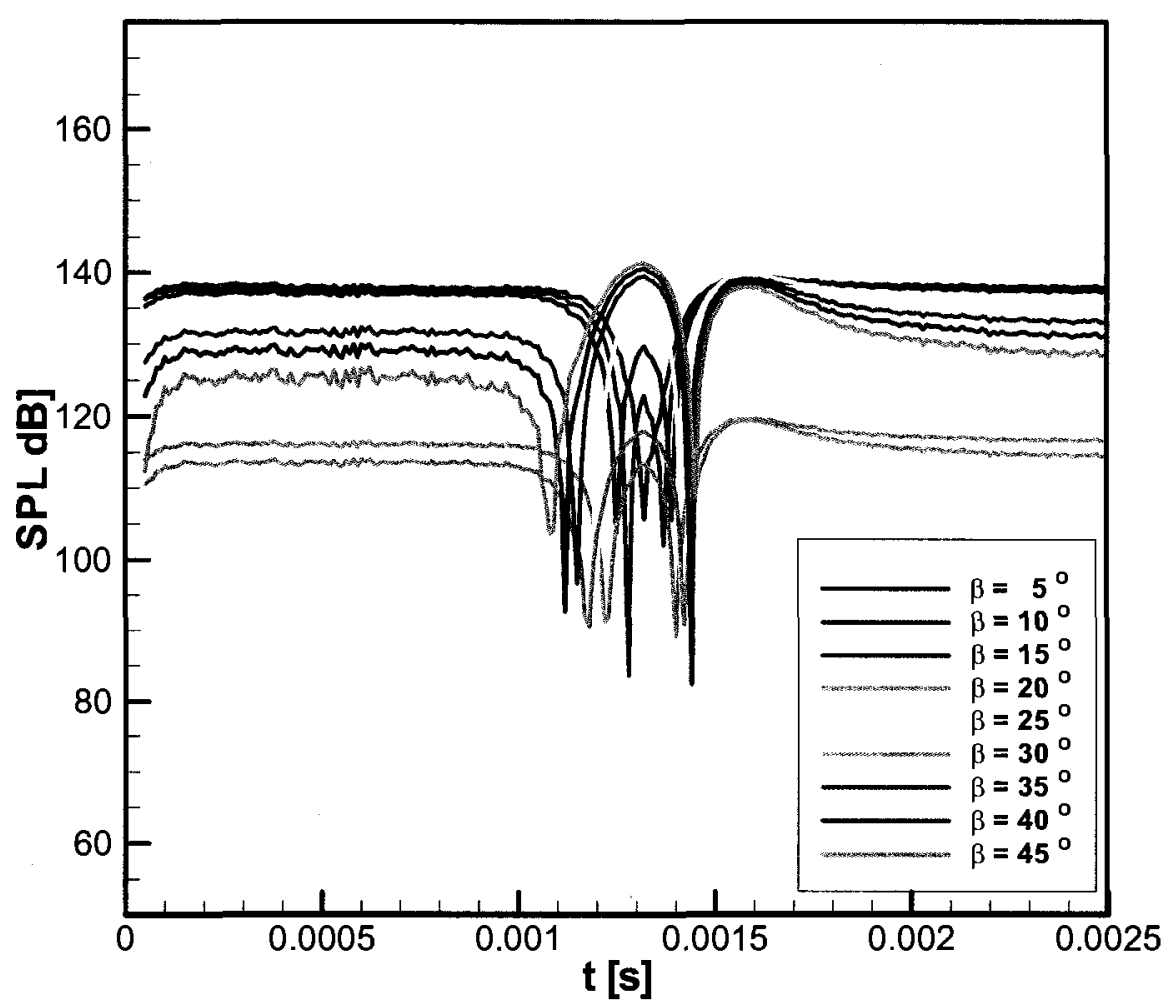

Figure 5.40 Comparison of SPL, $\alpha=0^{\circ}, h=-0.00 \mathrm{~m}, \mathrm{x}=0.15 \mathrm{~m}$. 


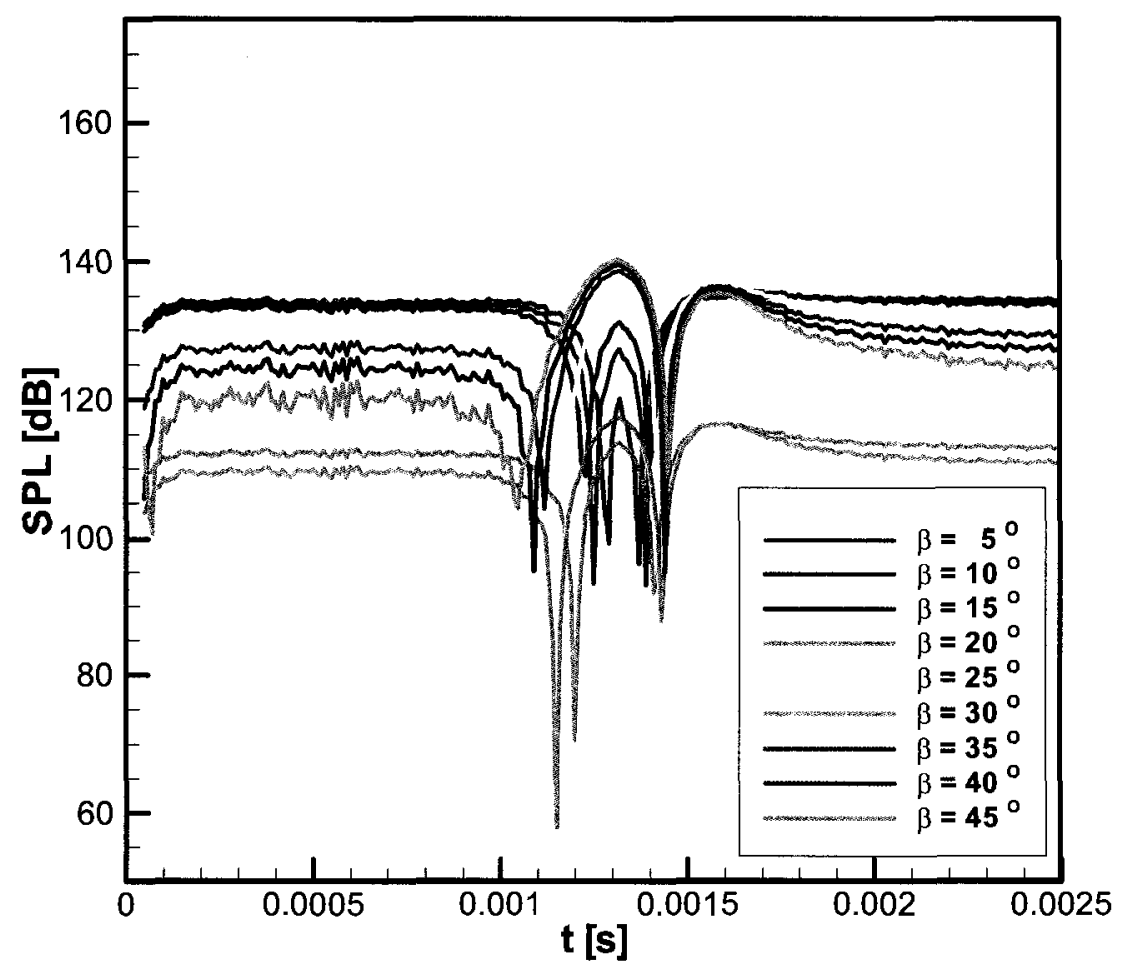

Figure 5.41 Comparison of SPL, $\alpha=0^{0}, h=-0.00 \mathrm{~m}, \mathrm{x}=\mathbf{0 . 2 \mathrm { m }}$.

Figures 5.40 and 5.41 presents the time-varying SPL for two different points, located at the horizontal distances from the leading edge $\mathrm{x}=-0.15 \mathrm{~m}$ and $\mathrm{x}=-0.2 \mathrm{~m}$.

These two points are located behind the releasing location of the vortex and, therefore, they do not sense the vortex-generated acoustic noise since they are not either on or near by the traveling path of the vortex. However, these two points still sense the BVI noise as seen in Figures 5.40 and 5.41.

Already knowing that the SPL values decrease with the increase of spanning angle, it is of particular interest to investigate the influence of airfoil-vortex vertical miss-distance on the SPL behaviour, along a path described by its corresponding spanning angle. The 
following analysis investigates the variation of SPL along four different paths defined by the spanning angles $\beta=10^{\circ}, \beta=20^{\circ}, \beta=30^{\circ}$ and $\beta=40^{\circ}$ respectively, for a fixed horizontal location $\mathrm{x}=-0.1 \mathrm{~m}$. Figure 5.42 presents the time variation of SPL corresponding to a spanning angle $\beta=10^{\circ}$, for six different vertical miss-distances (h).

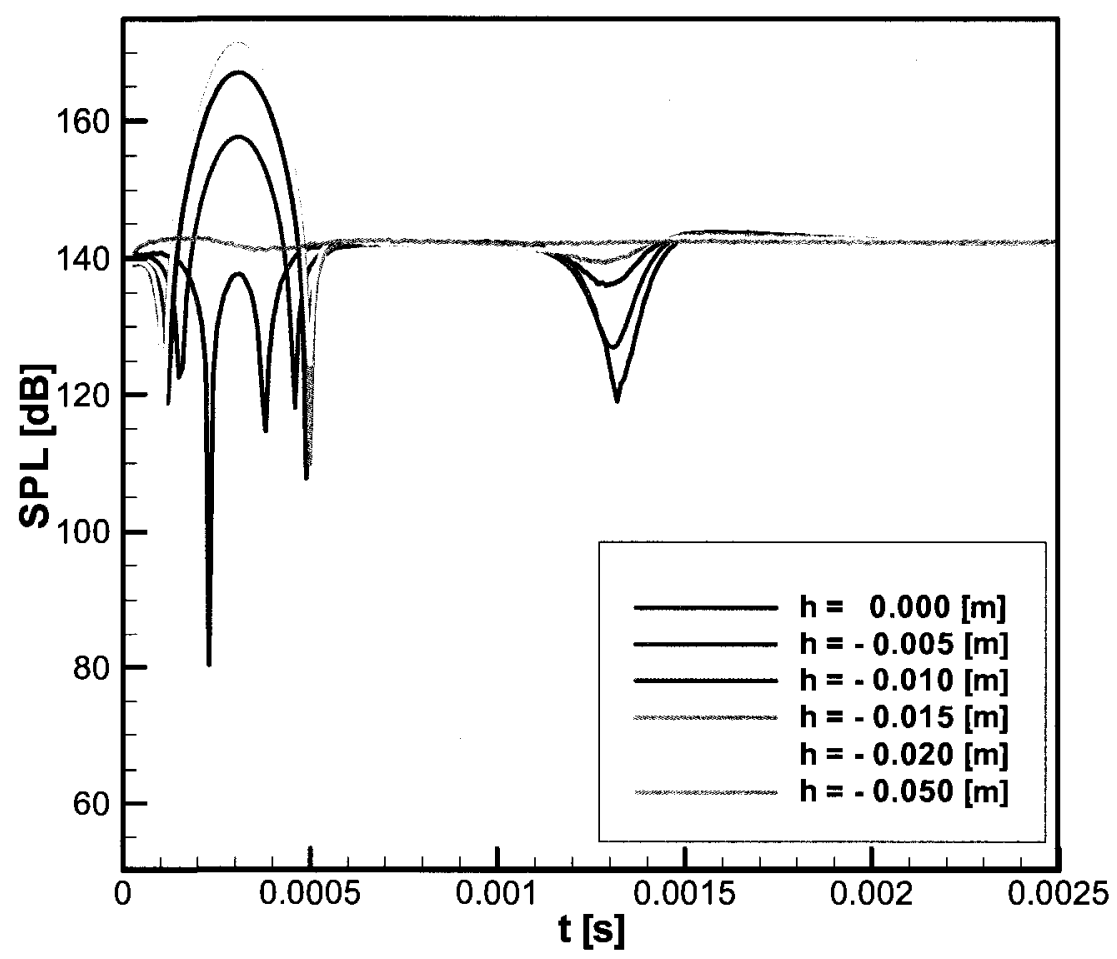

Figure 5.42 Comparison of SPL, $\alpha=0^{0}, \beta=10^{\circ} \mathrm{x}=\mathbf{- 0 . 1} \mathrm{m}$.

From Figure 5.42 it can be seen that SPL presents impulsive "spikes". The first SPL impulsive "spikes", occurring at about $t=0.0003 \mathrm{~s}$, are due to the vortex itself, which induces a pressure gradient in the flow field; they are not related to the AVI phenomenon. These peaks are associated with the vortex-generated acoustic noise as the vortex travels in the proximity of the interrogation points. The peaks associated with the vortex-generated acoustic 
noise increase with the increase of the airfoil-vortex vertical miss-distance and this can be explained as follows.

Using equation (5.4), the vertical coordinate of the interrogation point is $y=-0.017 \mathrm{~m}$. It is expected that the interrogation point sense the vortex-generated acoustic noise generated by the all vortices whose traveling paths are close to it. Hence, increasing the airfoil-vortex vertical miss-distance, the SPL values associated with the vortex-generated acoustic noise increase as well. The vertical miss-distances $h=-0.015 \mathrm{~m}$ and $\mathrm{h}=-0.02 \mathrm{~m}$ are the ones that sense the most intense vortex-generated acoustic noise. It is worth to notice that these two vertical miss-distances are somehow equidistantly located with respect to the interrogation point and, therefore, they generate the same SPL values associated with the vortex-generated acoustic noise. The SPL peaks occurring at the instant $t=0.0013 \mathrm{~s}$ are due to the AVI noise, and it can be seen that they decrease as the airfoil-vortex vertical miss-distance increases since the airfoil-vortex interactions become less and less severe. From Figure 5.42 it can be seen that as the airfoil-vortex vertical miss-distance increases, the amplitudes of the SPL decrease, due to the fact that increasing the vertical miss-distance the interaction between vortex and airfoil is less severe. The SPL amplitudes are the largest for the vertical missdistance $\mathrm{h}=0.00 \mathrm{~m}$ since the airfoil-vortex interaction is the most severe. Also it can be seen that when the vertical miss-distance is relatively large $\mathrm{h}=-0.05 \mathrm{~m}$, the SPL amplitudes are very small since the airfoil-vortex interaction is less significant. 


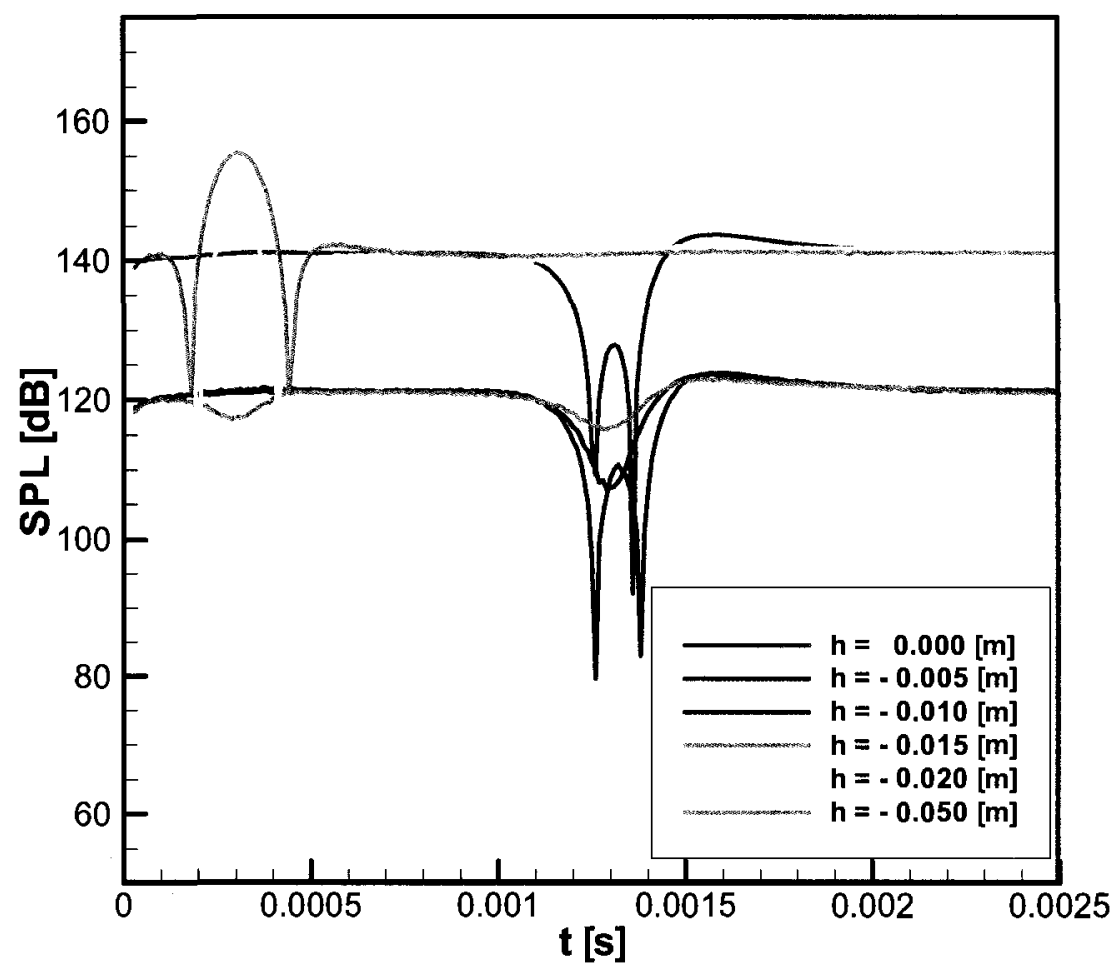

Figure 5.43 Comparison of SPL, $\alpha=0^{0}, \beta=20^{\circ} \mathrm{x}=-\mathbf{0 . 1} \mathrm{m}$.

Increasing further the spanning angle to $\beta=20^{\circ}$, the vertical location of the interrogation point also increases and this is reflected in the SPL values as well as seen in Figure 5.43. The vertical coordinate, corresponding to the horizontal distance $\mathrm{x}=-0.1 \mathrm{~m}$ is $\mathrm{y}=-0.0364 \mathrm{~m}$. The interrogation point having the coordinates $(-0.1,-0.0364)$ senses only the vortex-generated acoustic noise due to the vortices traveling on the paths $\mathrm{h}=-0.02 \mathrm{~m}$ and $\mathrm{h}=-0.05 \mathrm{~m}$. The vortex traveling on the path $\mathrm{h}=-0.05 \mathrm{~m}$ generates larger peaks due to the fact that its path is closer to the interrogation point. Further increase of the spanning angle to $\beta=30^{\circ}$, corresponds to increase vertical coordinate to $y=-0.057$. 


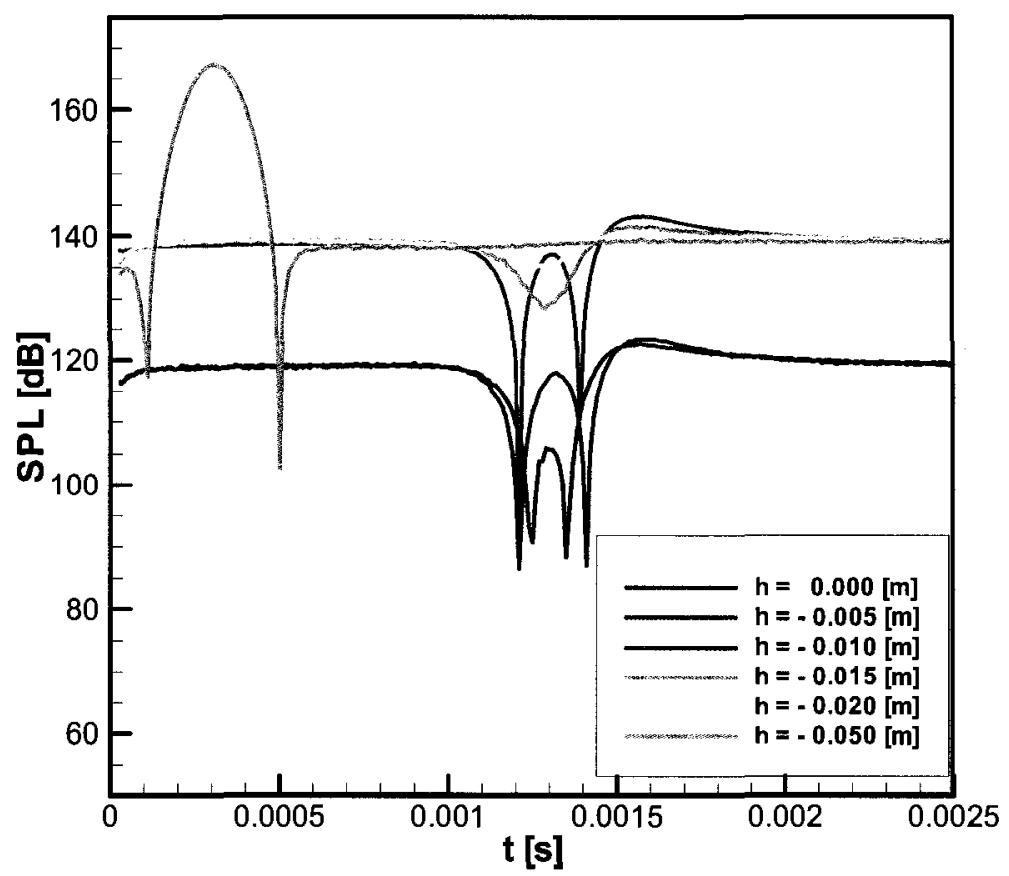

Figure 5.44 Comparison of SPL, $\alpha=\mathbf{0}^{0}, \beta=30^{\circ} \mathrm{x}=-\mathbf{0 . 1} \mathrm{m}$.

As expected the vortex traveling on the path $h=-0.05 \mathrm{~m}$ generates the largest SPL peaks, Figure 5.44. However, all the interrogation points sense the AVI noise. Similar behaviour of SPL was observed for a spanning angle $\beta=40^{\circ}$. The corresponding vertical coordinate is $\mathrm{y}=$ - 0.0839. In this case, the interrogation point does not sense the vortex-generated acoustic noise since its location is far away from all vortex traveling paths. However, the interrogation point senses the BVI noise at the instant $t=0.0013 \mathrm{~s}$, as seen in Figure 5.45. 


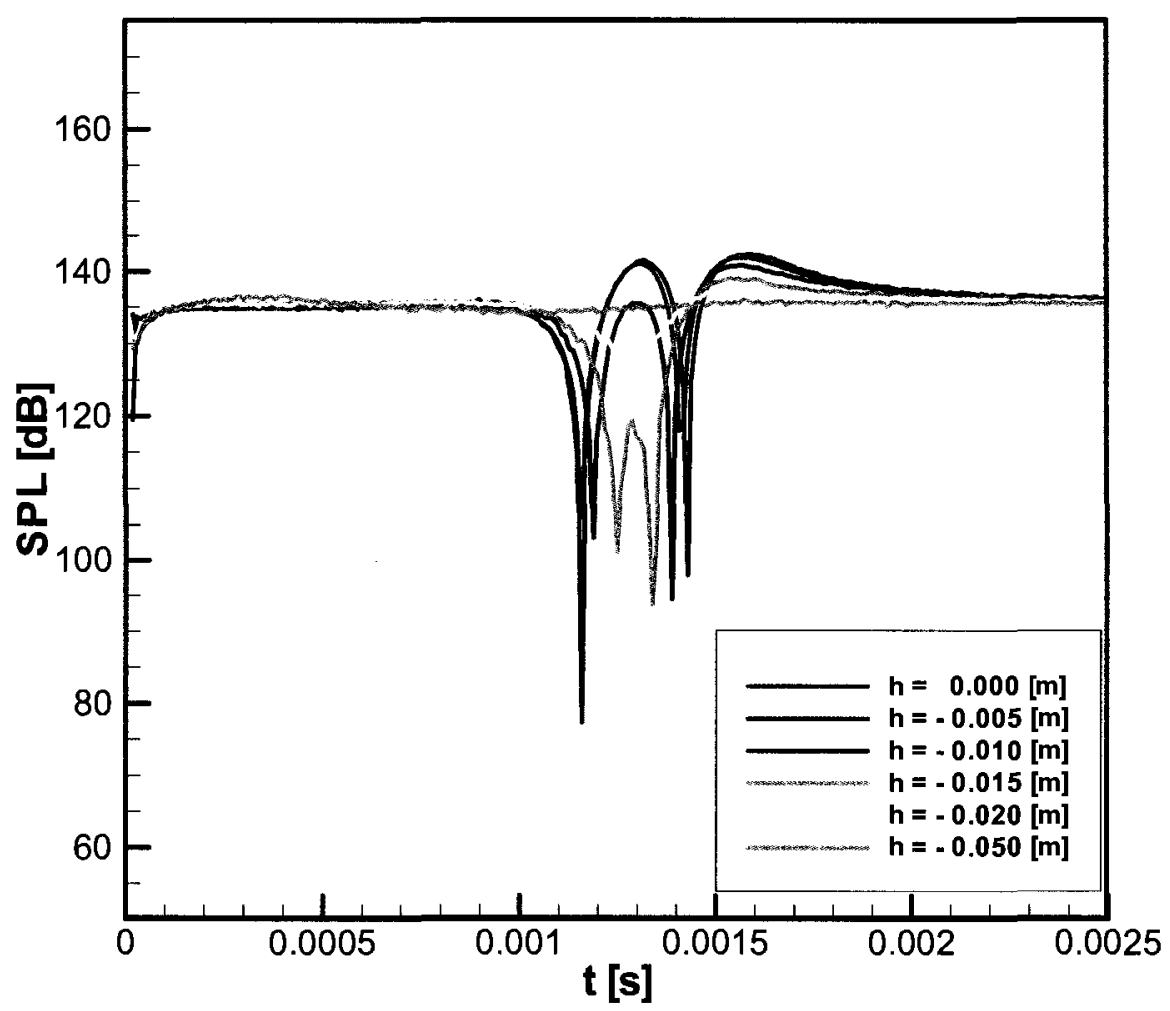

Figure 5.45 Comparison of SPL, $\alpha=0^{0}, \beta=40^{\circ} \mathrm{x}=-\mathbf{0 . 1} \mathrm{m}$.

From the present analysis, it can be concluded that for a given vortex strength, the missdistance determines the disturbance velocity sensed at the blade. Hence, with the increase of the vertical miss-distance, a decrease of the disturbance velocity and the resulting AVI noise is observed. Note that the sound pressure is proportional to the time-rate of change of local blade loading. Therefore, the sound pressure decreases with increasing miss-distance due to both the decreased disturbance strength and the stretched time scale associated with a larger miss-distance. 
Knowing how the airfoil-vortex vertical miss-distance influences the acoustic field, it is important to investigate the influence of angle of attack on the acoustic field. With this objective, a further aeroacoustic investigation was conducted.

Analog to the test case of airfoil at angle of attack $\alpha=0^{0}$, similar trend of timevarying SPL was observed for the angle of attack $\alpha=5^{0}$.

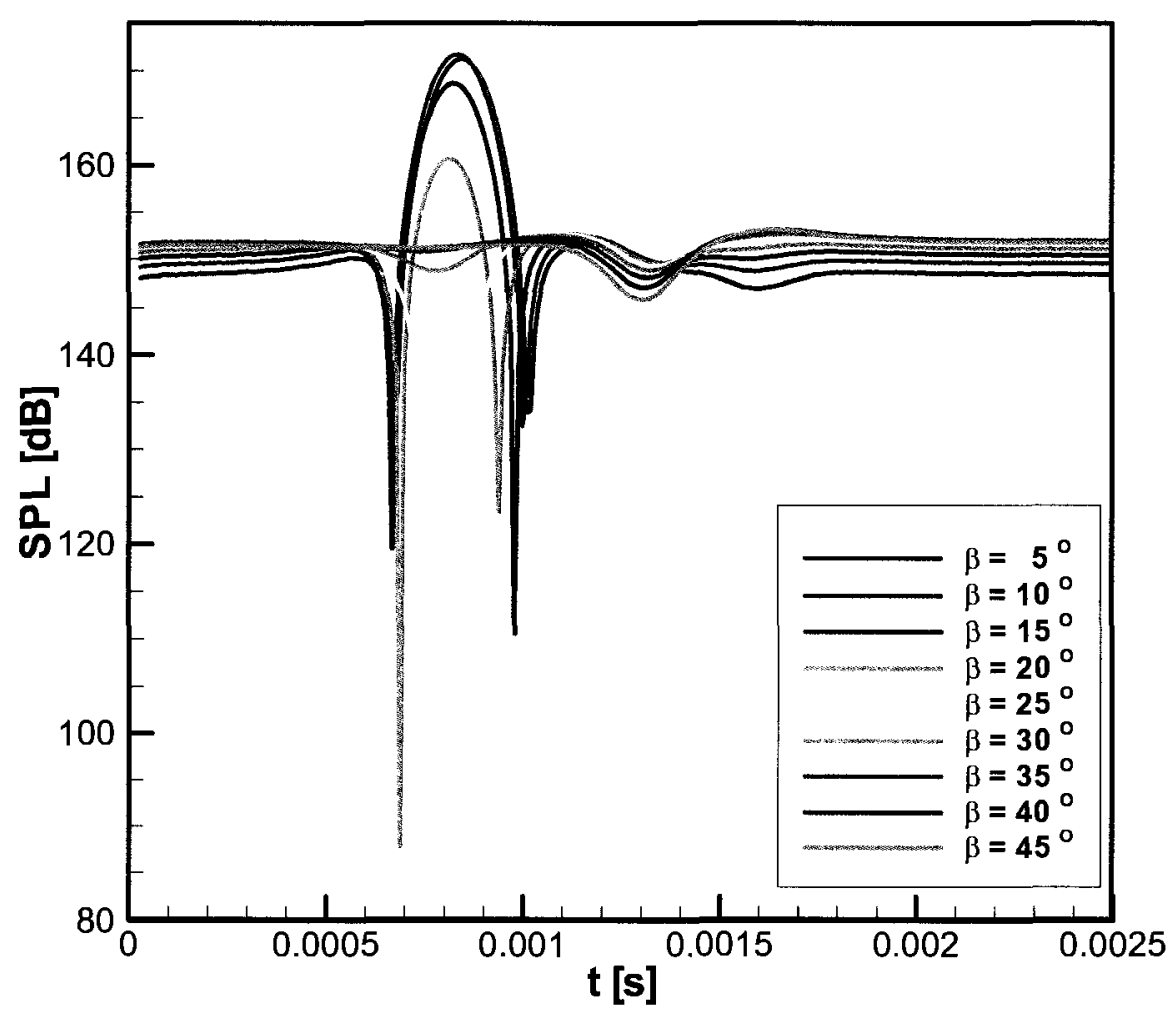

Figure 5.46 Comparison of SPL, $\alpha=5^{0}, h=-0.00 \mathrm{~m}, \mathrm{x}=-\mathbf{0 . 0 5 m}$.

A decrease of SPL peaks at the instant when the vortex encounters the airfoil was observed as the airfoil angle of attack increases, Figure 5.46. This is a result of the airfoil-vortex mechanism of interaction. The vortex encounters the airfoil at an angle of attack and this attenuates the airfoil-vortex mechanism of interaction and implicitly the effect of the vortex 
on the flow field and SPL. Although the SPL peaks at the instant of airfoil-vortex interaction are smaller for an angle of attack $\alpha=5^{\circ}$, the overall SPL values are higher, compared to those of angle of attack $\alpha=0^{\circ}$. As a general trend of SPL, it is observed that the vortex-generated acoustic noise is most sensed by the points located in the proximity of the vortex travel path, and the overall SPL values decrease with the increase of the spanning angle. This observation is similar with the test case of airfoil at angle of attack $\alpha=0^{\circ}$.

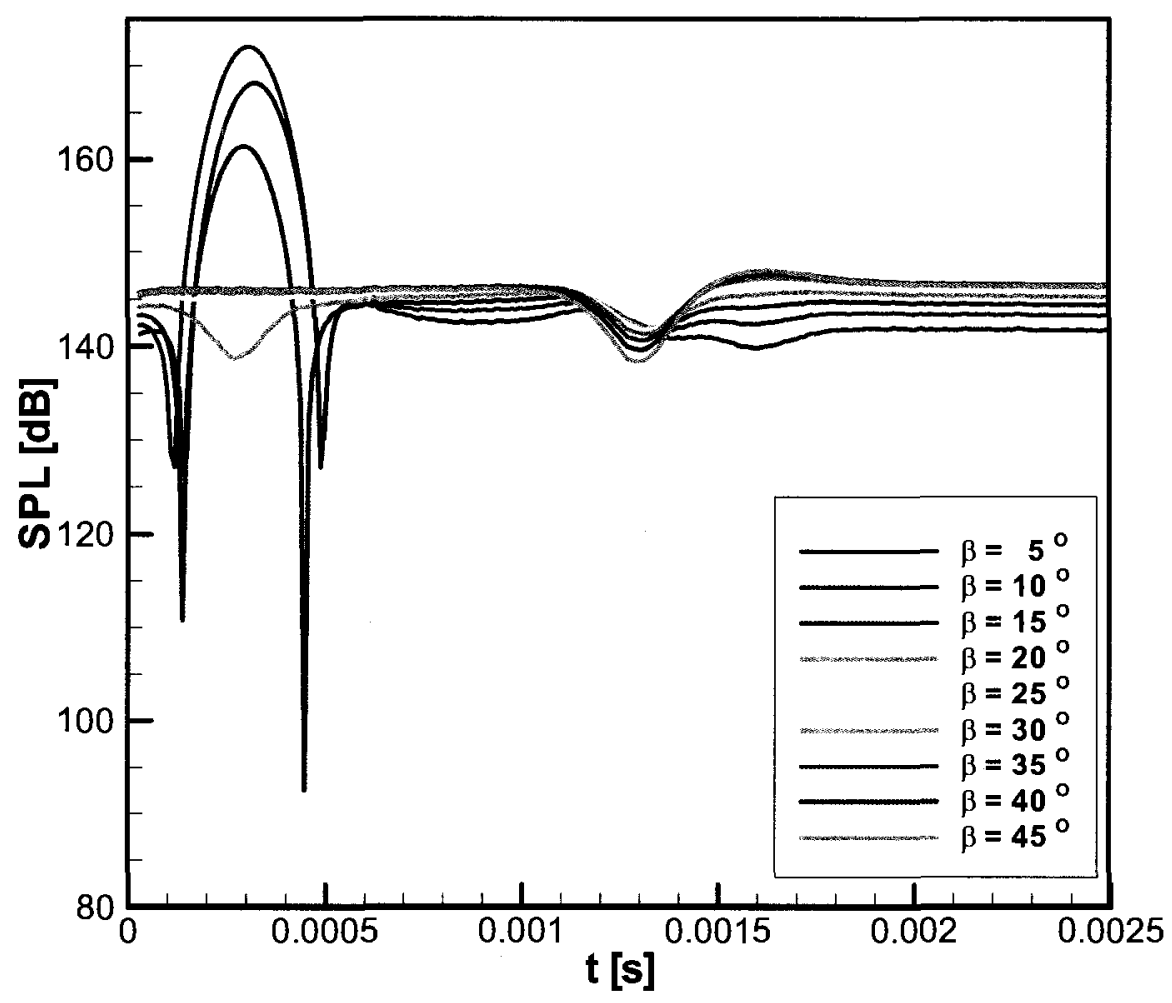

Figure 5.47 Comparison of SPL, $\alpha=5^{0}, \mathbf{h}=-\mathbf{0 . 0 0} \mathrm{m}, \mathrm{x}=\mathbf{- 0 . 1 \mathrm { m }}$. 


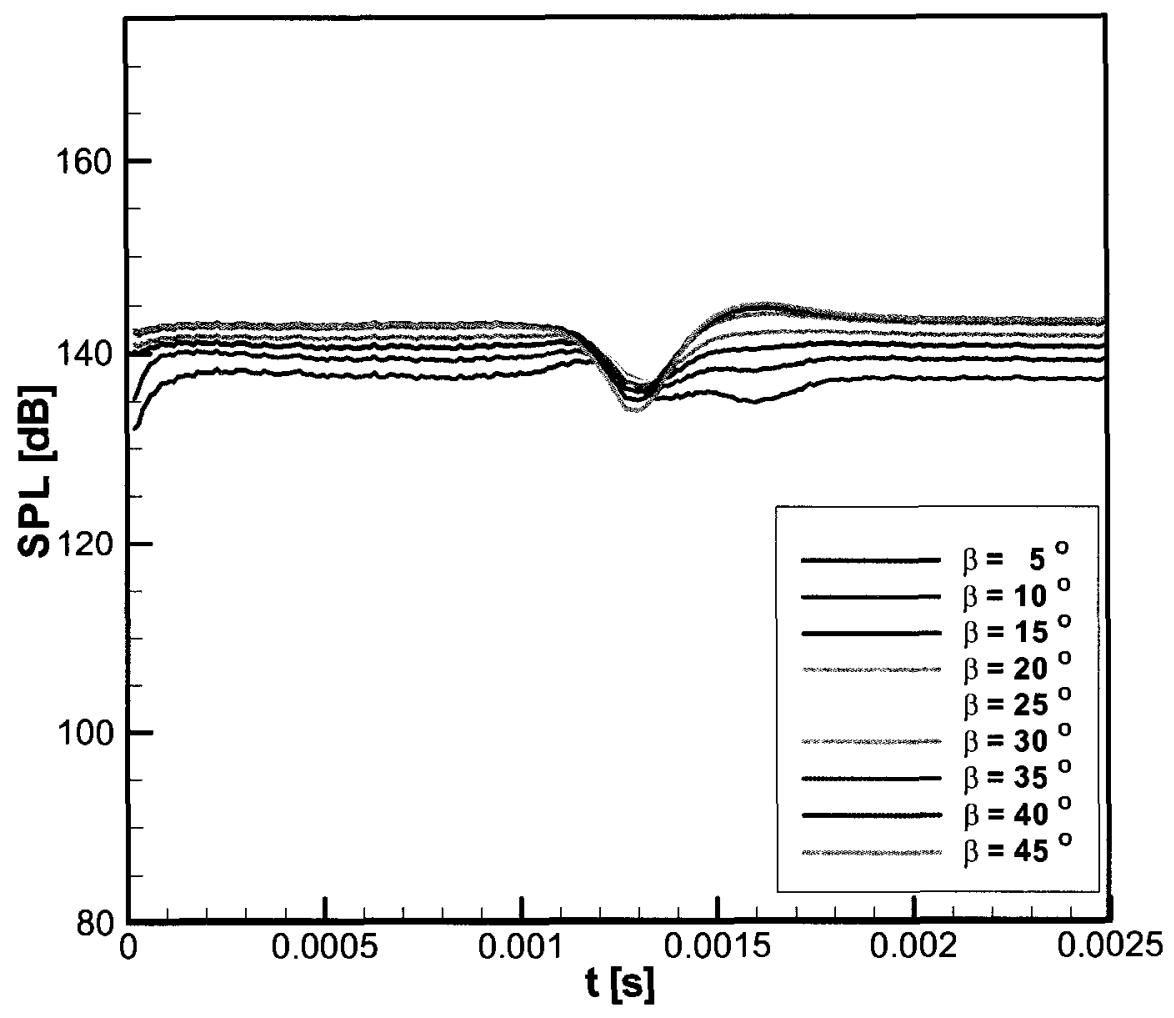

Figure 5.48 Comparison of SPL, $\alpha=5^{0}, \mathrm{~h}=\mathbf{- 0 . 0 0} \mathrm{m}, \mathrm{x}=\mathbf{- 0 . 1 5 m}$. 


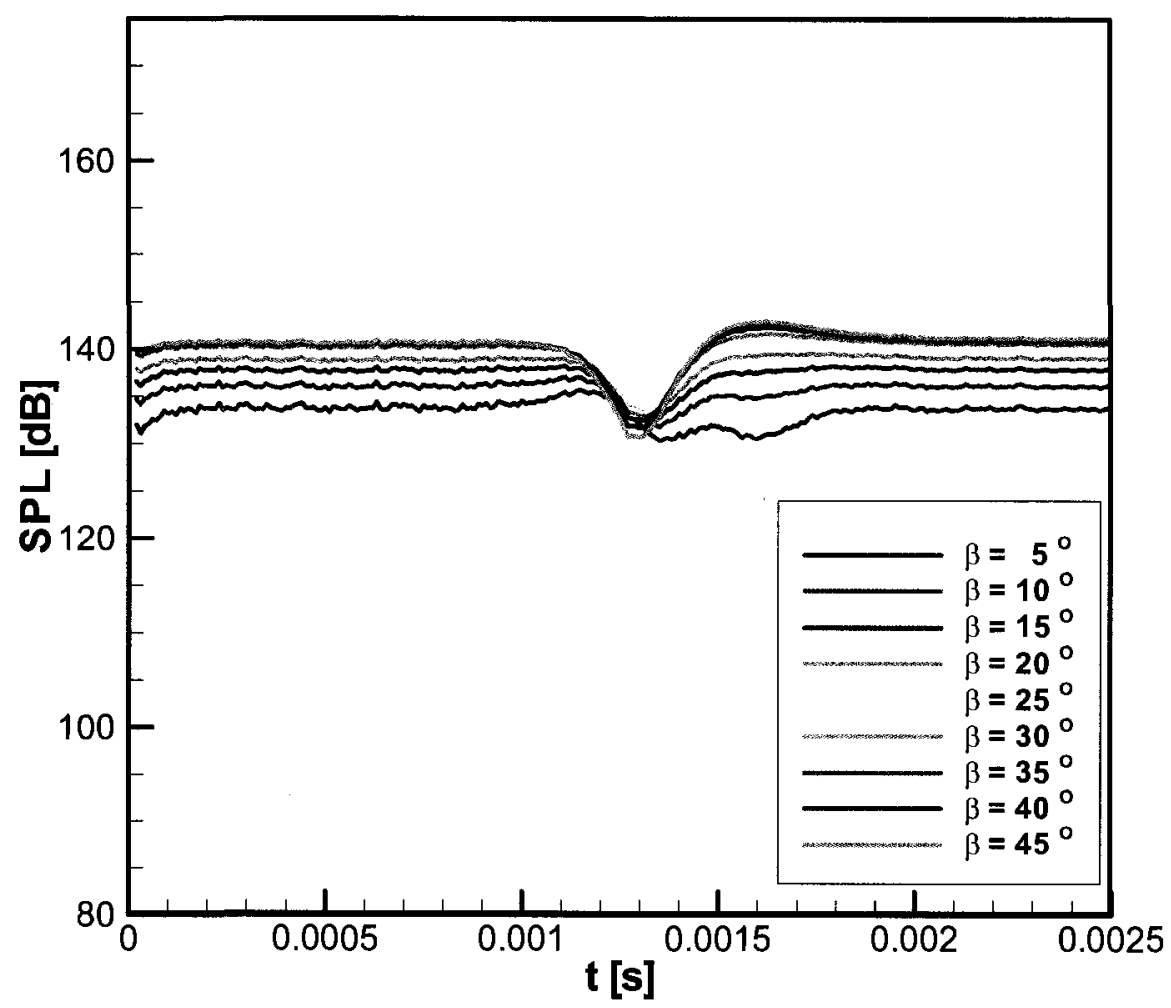

Figure 5.49 Comparison of SPL, $\alpha=5^{0}, \mathrm{~h}=-0.00 \mathrm{~m}, \mathrm{x}=-0.2 \mathrm{~m}$.

Figures 5.48 and 5.49 presents the time-varying SPL, corresponding to an airfoil angle of attack $\alpha=5^{0}$, for two different horizontal distances from the leading edge, $\mathrm{x}=-0.15 \mathrm{~m}$ and $\mathrm{x}$ $=-0.2 \mathrm{~m}$. Similar to the test case of airfoil at angle of attack $\alpha=0^{0}$, the interrogation points do not sense the vortex-generated acoustic noise since they are far away from the vortex traveling path. They sense, though, the BVI noise. Lower values of the SPL associated with the AVI noise are observed when compared with the corresponding interrogation points for an angle of attack $\alpha=0^{\circ}$. These lower values are due to the influence of angle of attack on the airfoil-vortex mechanism of interaction. 


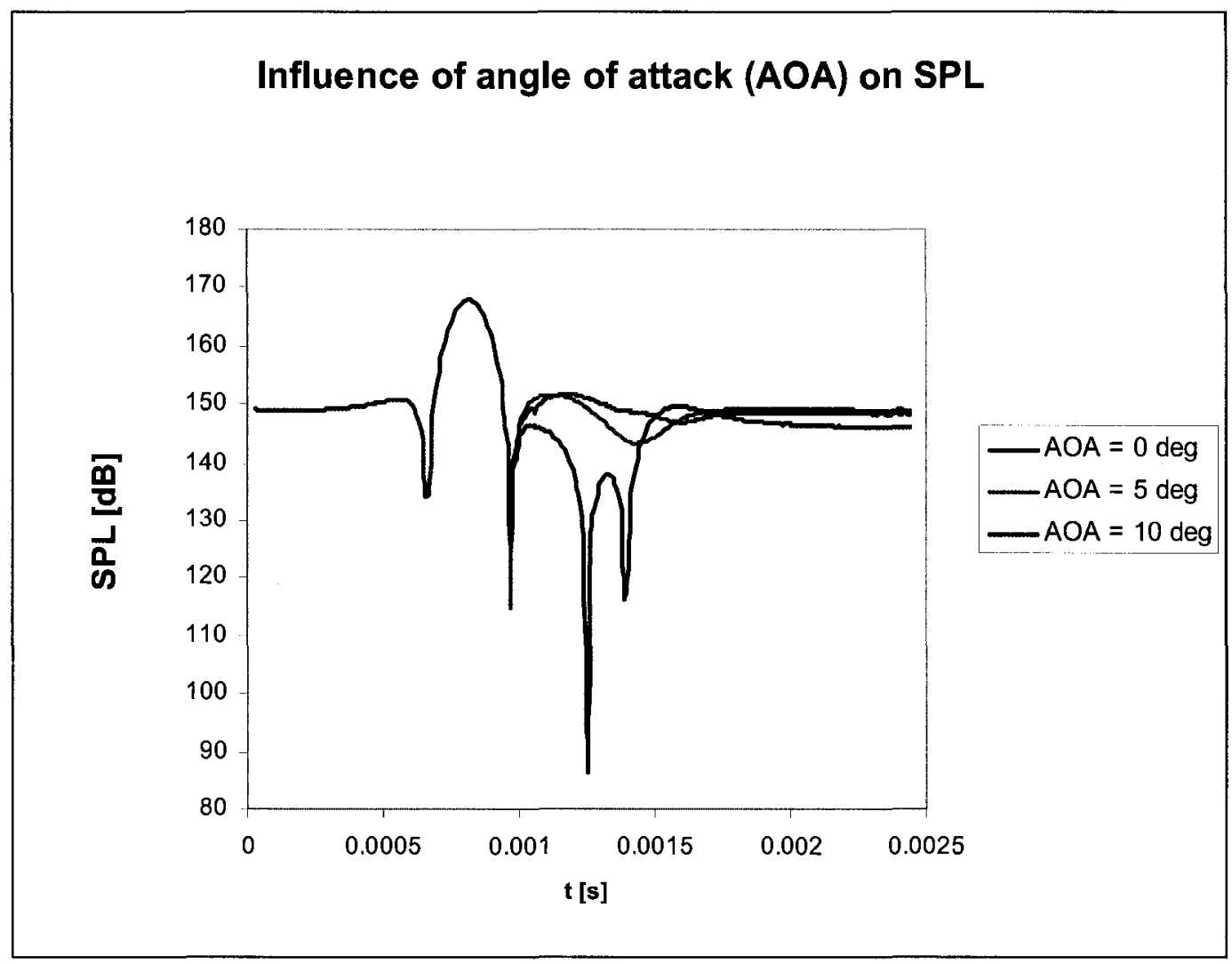

Figure 5.50 Comparison of SPL for different angles of attack, $h=-0.00 \mathrm{~m}, \mathrm{x}=-\mathbf{0 . 0 5 m}$.

Figure 5.50 presents the comparison of time-varying SPL for three different angles of attack. From Figure 5.50 it can be seen that similar to the time-varying aerodynamic coefficients, the values of SPL associated with AVI decrease with the increase of angle of attack as seen at the instant $t=0.0013 \mathrm{~s}$. The amplitudes of SPL decrease significantly with the increase of angle of attack and this suggests that the increase of angle of attack attenuates the AVI acoustic noise. This is explained by the fact that in general BVI is more significant in low speed descent when the airfoil angle of attack is low as preparation for landing takes place. 


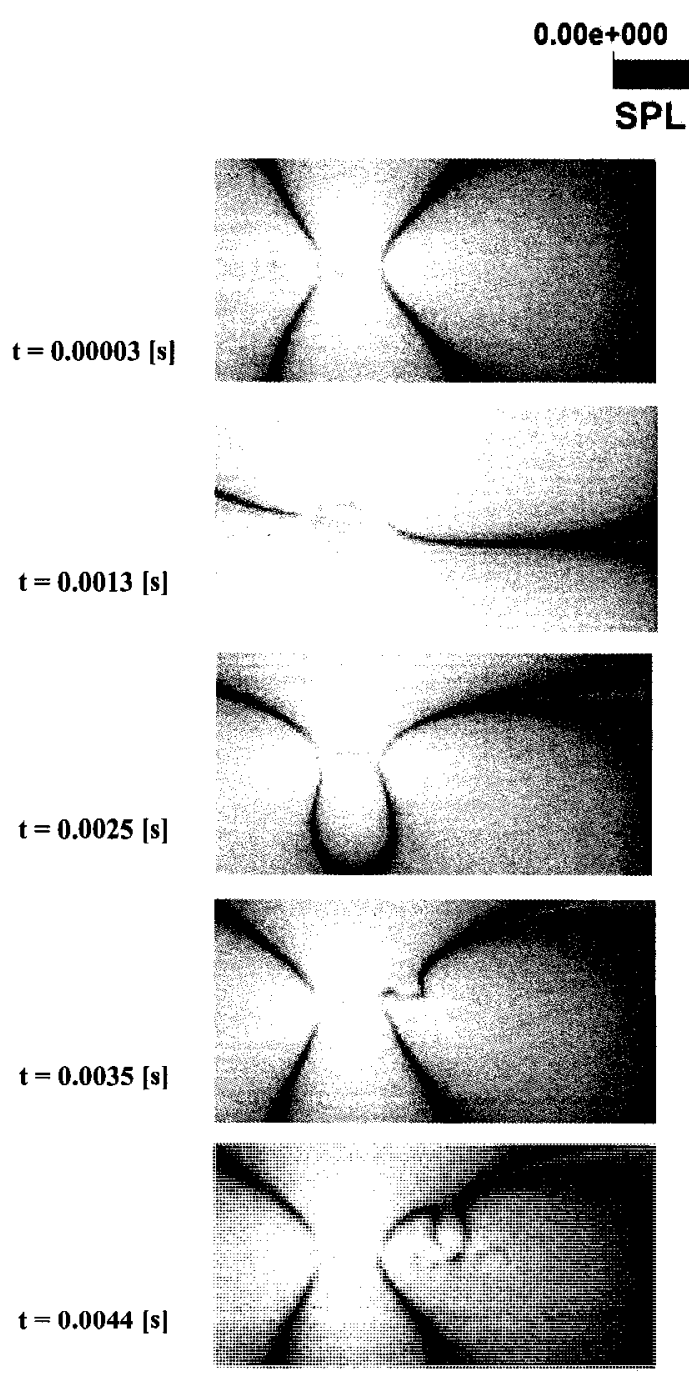

a) $r=0.5 c$
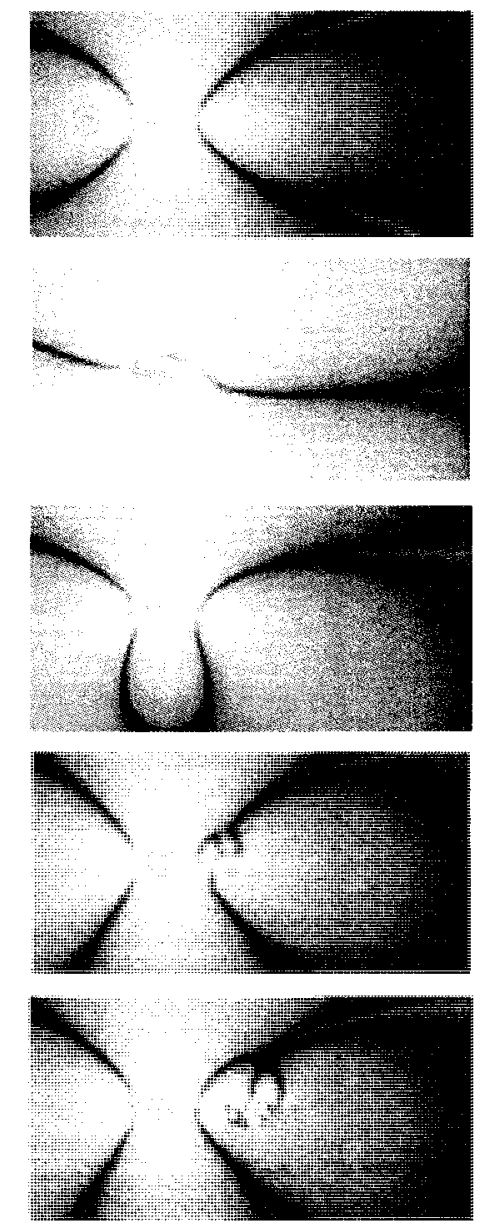

b) $\mathrm{r}=0.4 \mathrm{c}$
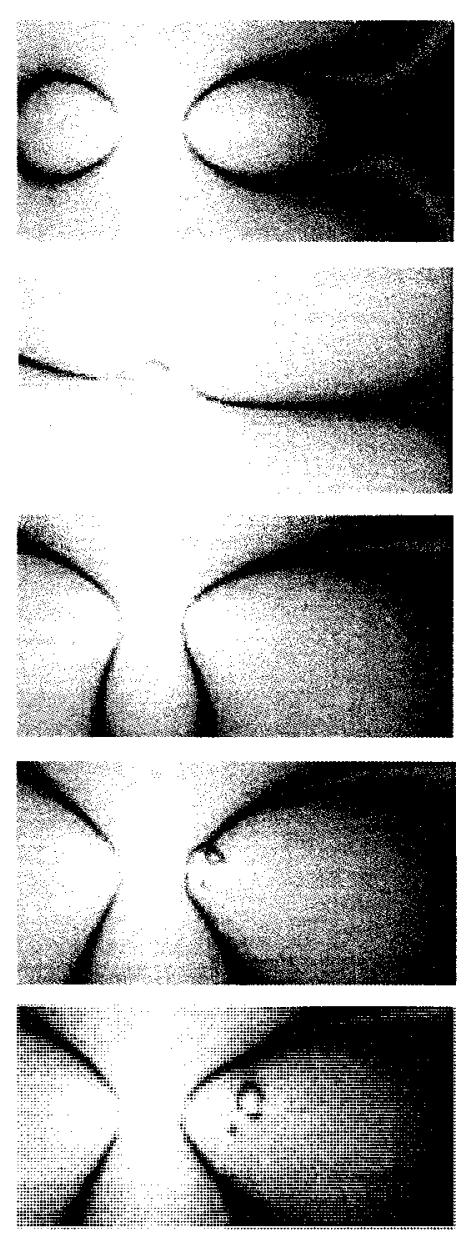

c) $\mathrm{r}=0.3 \mathrm{c}$

Figure 5.51 Time history of SPL, for different vortex core sizes.

The investigation regarding the influence of angle of attack on the SPL shows that the angle of attack not only affects the aerodynamic coefficients but the acoustic field as well. The increase of angle of attack attenuates the airfoil-vortex interactions and implicitly decrease the SPL values, at the instant of airfoil-vortex interaction.

A similar analysis, having as goal the investigation of vortex characteristics on the acoustic field, was conducted and presented in the following. As it was observed in the 
previous section, as a result of the influence of vortex core size on the flow field, the aerodynamic coefficients were affected as well. Hence, it is anticipated that the vortex core size would also affect the acoustic field. It is of interest to physically analyze and understand these effects.

Figure 5.50 presents the time-varying sound pressure level (SPL) at five different instants in time for three different vortex core sizes. For all test cases, contour lines of constant magnitude $(100 \mathrm{~dB})$ can be observed. The profile of these counter lines depends on the vortex core size, as seen at the instant $t=0.00003 \mathrm{~s}$. For the case of a vortex core size $r=$ $0.5 \mathrm{c}$, at the instant $\mathrm{t}=0.00003 \mathrm{~s}$, the contour lines describe a hyperbola with the foci located on the camber line of airfoil. As the vortex core size decreases, the hyperbola branches distort and the contour lines define circular shapes, Figure $5.50 \mathrm{c}, \mathrm{t}=0.00003 \mathrm{~s}$. The constant SPL contour lines exhibit a transient behaviour in time. It follows that, as the vortex approaches the airfoil, the upper branches of hyperbola (above camber line) merge together and generate a convex bell shape contour whose size decreases as the vortex encounters the airfoil.

The constant SPL contour reaches a minimum at the instant when the vortex encounters the airfoil, $\mathrm{t}=0.0013 \mathrm{~s}$. As the vortex passes the leading edge there is a sudden change in the SPL bell shape contour, becoming a concave bell shape as a result of merging process between the lower branches of hyperbola, as seen at the instant, $t=0.0025 \mathrm{~s}$. As the residual component of the vortex leaves the airfoil, the contour lines define a hyperbolic profile. Although the SPL contour profile exhibits very similar pattern for all test cases, there are still differences at the instant when the residual component of the vortex merges into the airfoil wake, $\mathrm{t}=0.0035 \mathrm{~s}$. It can be seen that the residual component of the vortex distorts the 
SPL front described, by the upper branch of hyperbola, in a different manner at the instant when leaves the airfoil, $\mathrm{t}=0.0035 \mathrm{~s}$. This result is associated with the airfoil-vortex mechanism of interaction since the characteristics of the residual component of the vortex depend on the airfoil-vortex mechanism of interaction.

As the residual component of the vortex travels far downstream, the SPL contour lines define a hyperbolic profile of constant magnitude, for all test cases. Similar to previous test cases an aeroacoustic investigation based on time-varying SPL is conducted.

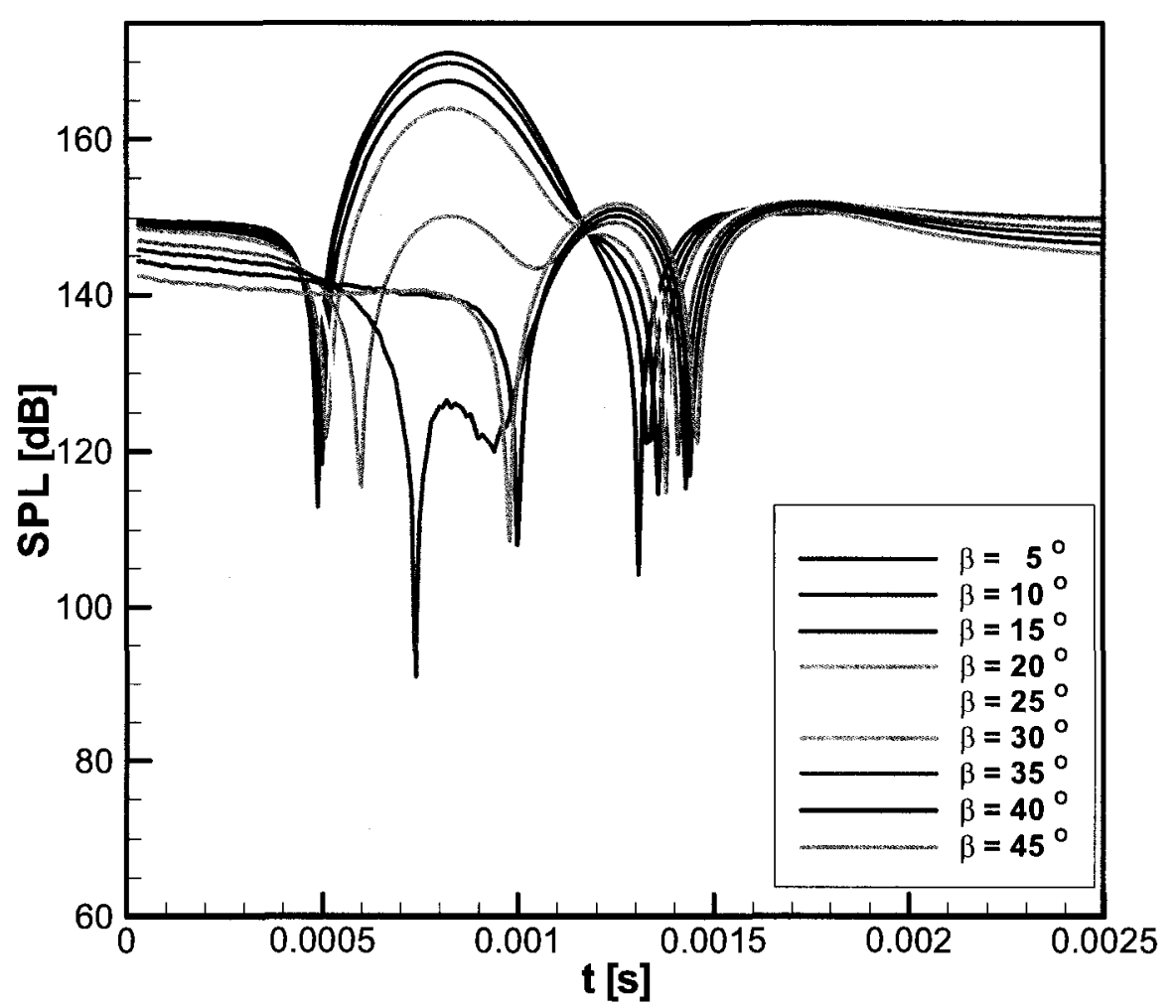

Figure 5.52 Comparison of SPL, $r=0.5 c, \alpha=0^{0}, h=-0.00 \mathrm{~m}, \mathrm{x}=-\mathbf{0 . 0 5} \mathrm{m}$. 


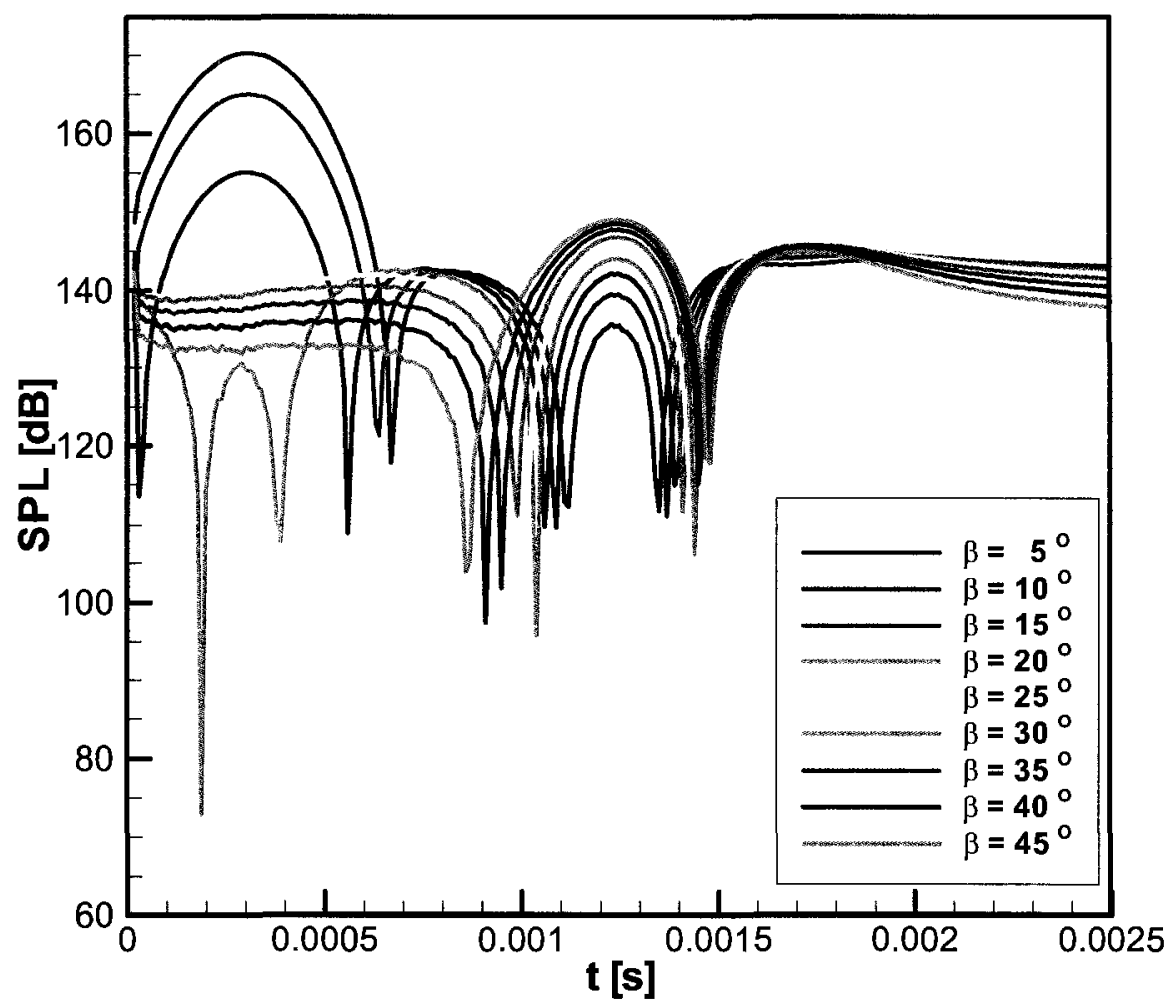

Figure 5.53 Comparison of SPL, $r=0.5 c, \alpha=0^{0}, h=-0.00 \mathrm{~m}, \mathrm{x}=-0.1 \mathrm{~m}$.

From Figure 5.51 it can be seen that when the interrogation point is located close to the leading edge there is an overlap of the SPL values due to the vortex-generated acoustic noise with the ones due to the AVI. As a result of the increasing spanning angle, the SPL peaks decrease and the parabola openings shrink, as seen in Figure 5.51. A shift in time of the SPL values associated with AVI acoustic is observed as well, at instant $t=0.0013 \mathrm{~s}$.

With the increase of horizontal distance from the leading edge, there is a clear distinction between the SPL values; the SPL values due to the AVI being identified at the instant $t=0.0013 \mathrm{~s}$, Figure 5.52. The peaks of the SPL associated with AVI acoustic noise 
increase with the increase of spanning angle. Also it can be noticed that the openings of the parabola associated with these SPL values enlarge with the increase of the spanning angle.

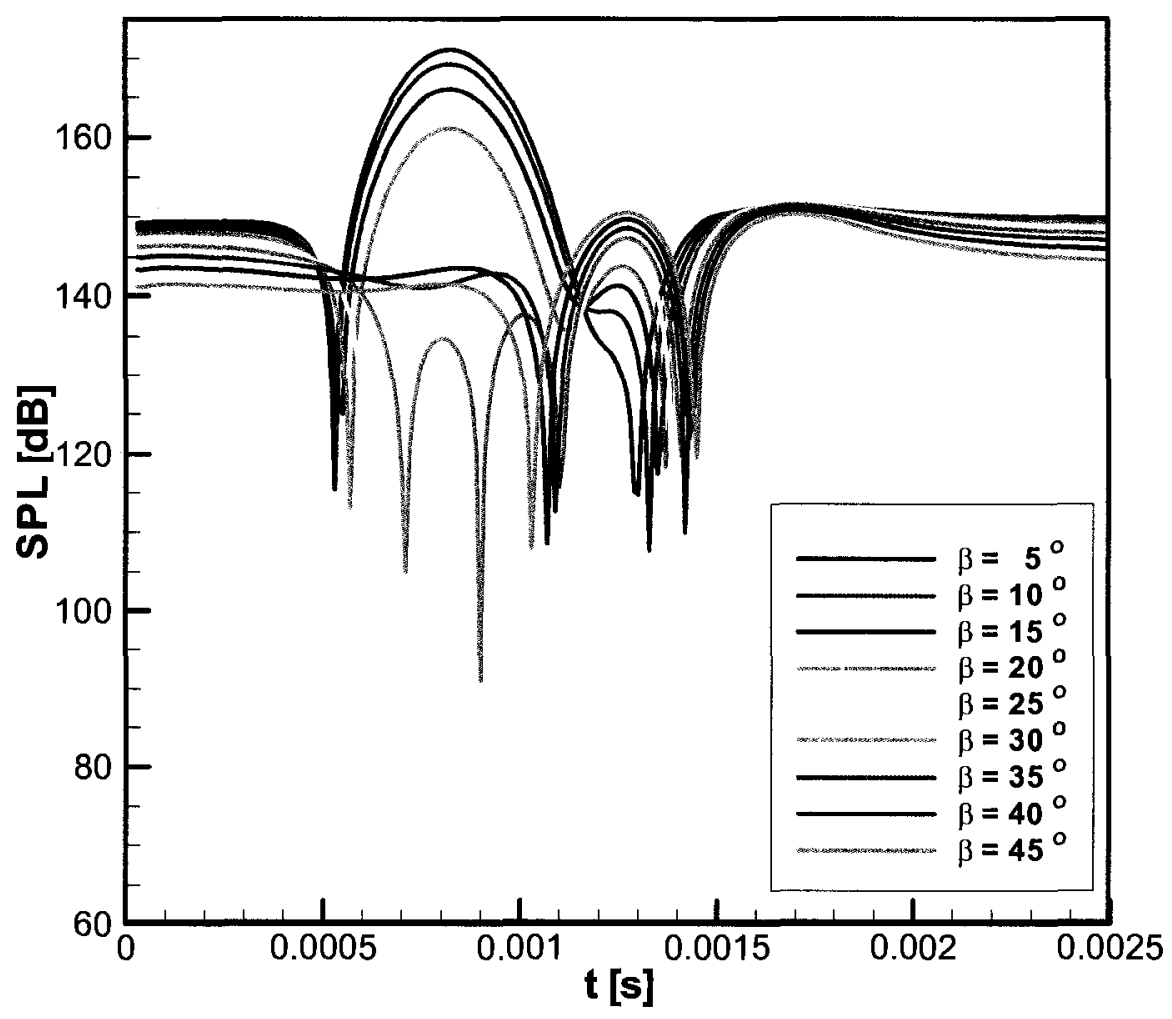

Figure 5.54 Comparison of SPL, $r=0.4 c, \alpha=0^{0}, h=-0.00 m, x=-0.05 m$. 


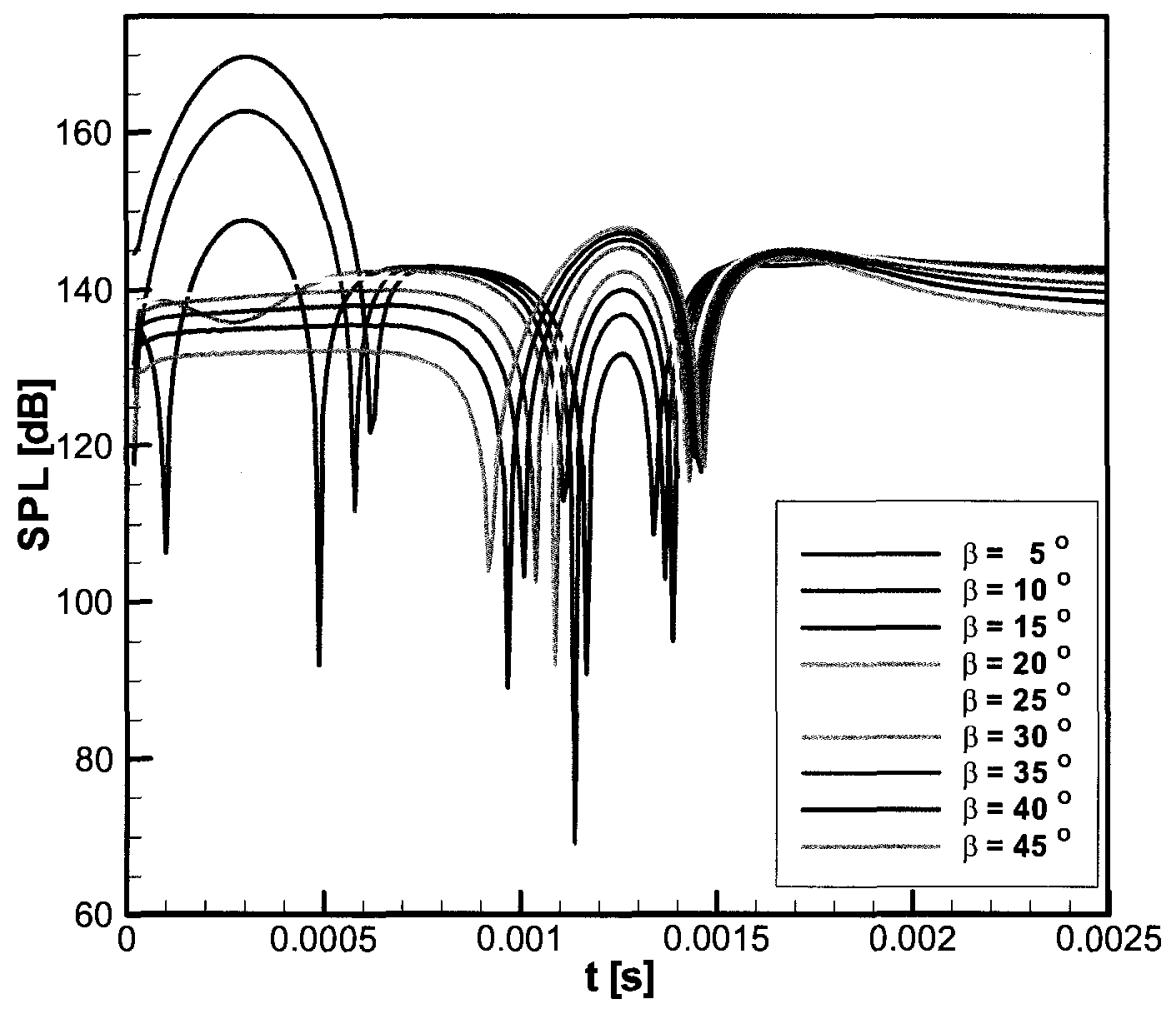

Figure 5.55 Comparison of SPL, $r=0.4 c, \alpha=0^{0}, h=-0.00 \mathrm{~m}, \mathrm{x}=-\mathbf{0 . 1 \mathrm { m }}$. 


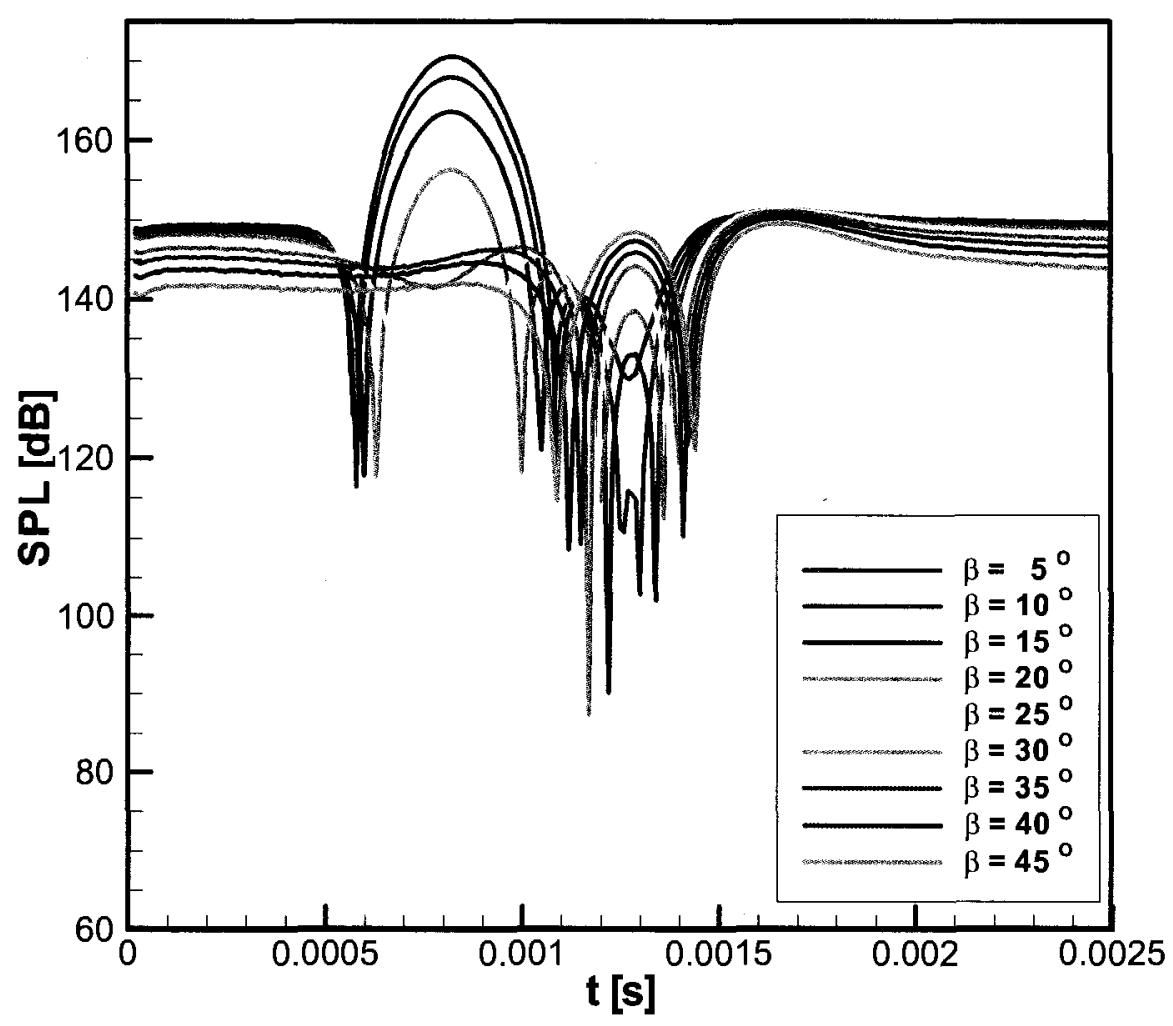

Figure 5.56 Comparison of SPL, $r=0.3 c, \alpha=0^{0}, h=-0.00 \mathrm{~m}, \mathrm{x}=-\mathbf{0 . 0 5 m}$.

Similar behaviour of SPL was observed for a vortex core radius $r=0.4 c$ and $r=0.3 c$. Figure 5.53 presents the SPL time variation at the fixed horizontal location, $\mathrm{x}=-0.05 \mathrm{~m}$, for nine different values of the spanning angle and for a vortex core radius $r=0.4 c$. From Figure 5.53 it can be seen that as the vortex core size decreases, the opening of the branches of parabola narrows, and this is a result of vortex core size effect on the SPL. As the vortex core size decreases, the range of vortex influence on the SPL values decreases as well. As it has been already stated, the decrease of vortex core size is associated with the narrowing of the opening of the parabola branches and this is a progressive process. Consequently, for the case 
of a vortex core size $r=0.3 \mathrm{c}$, the opening of the parabola branches narrows significantly, as seen in Figure 5.54 and Figure 5.55, respectively.

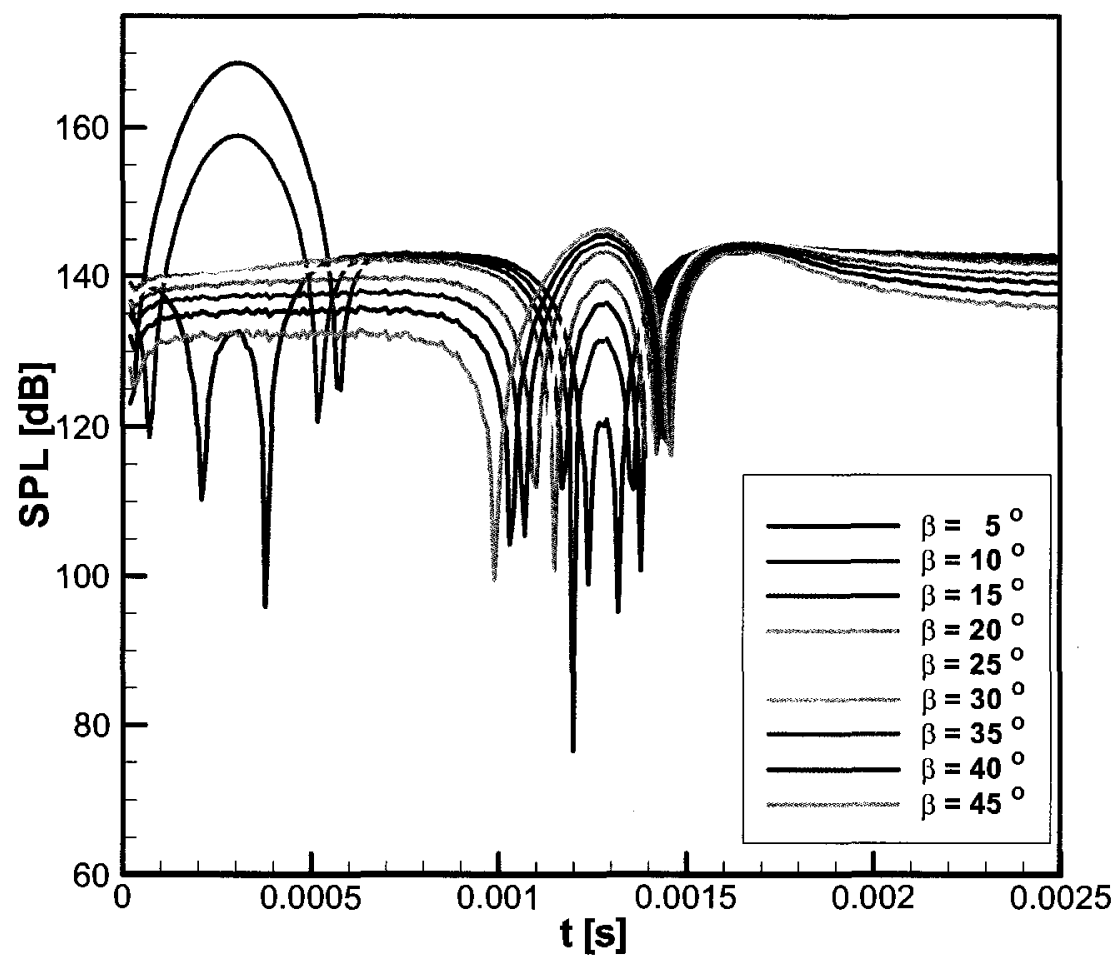

Figure 5.57 Comparison of SPL, $r=0.3 \mathrm{c}, \alpha=0^{0}, \mathrm{~h}=-0.00 \mathrm{~m}, \mathrm{x}=-0.1 \mathrm{~m}$.

Although the overall trend of SPL function of vortex core size shows a similar behaviour for all test cases, there are still some differences due to the airfoil-vortex mechanism of interaction. In this sense Figure 5.57, Figure 5.58, Figure 5.59 and Figure 5.60, respectively present a comparison of the SPL at two different points, for two different spanning angles $\beta=15^{\circ}$ and $\beta=30^{\circ}$. 


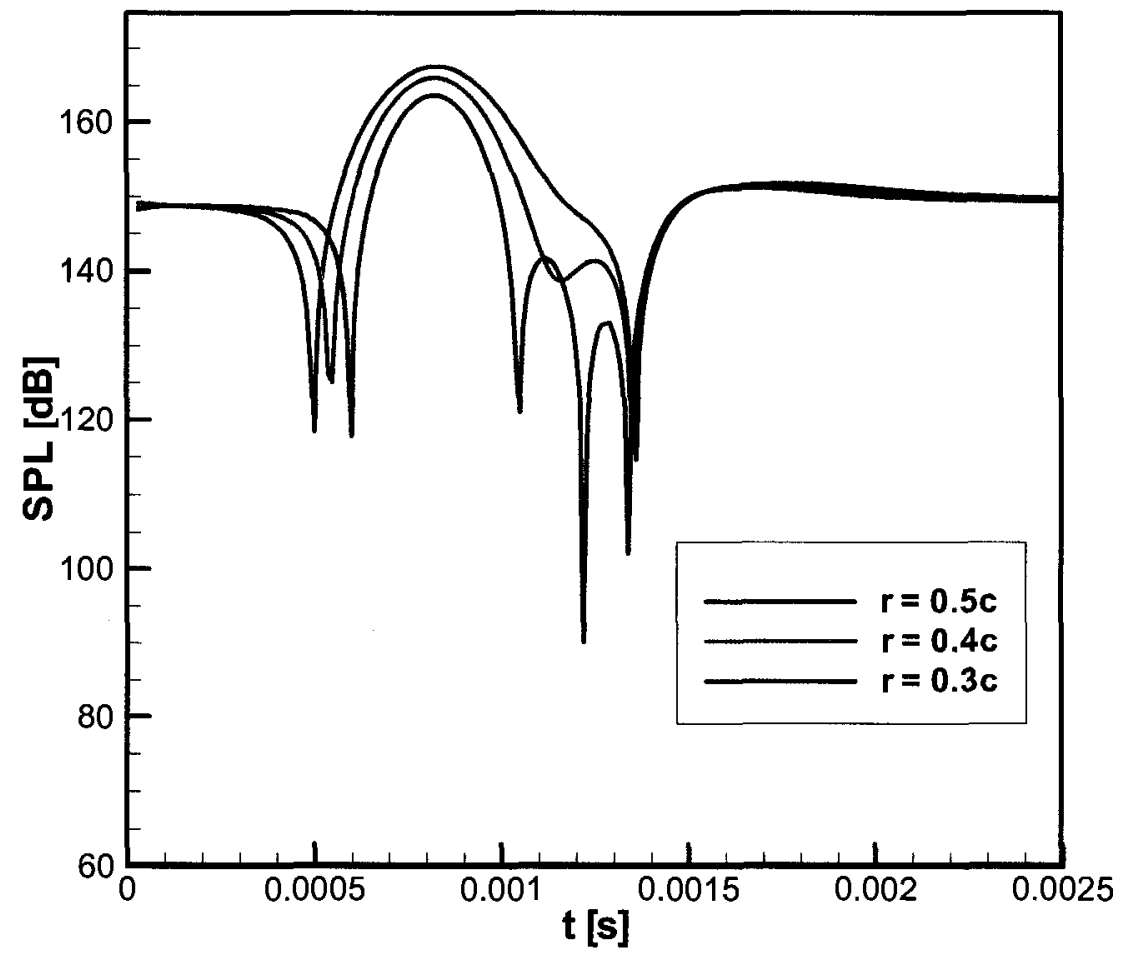

Figure 5.58 Comparison of SPL, $\alpha=\mathbf{0}^{\circ}, \mathbf{h}=-\mathbf{0 . 0 0} \mathrm{m}, \beta=15^{0}, \mathrm{x}=\mathbf{- 0 . 0 5 m}$. 


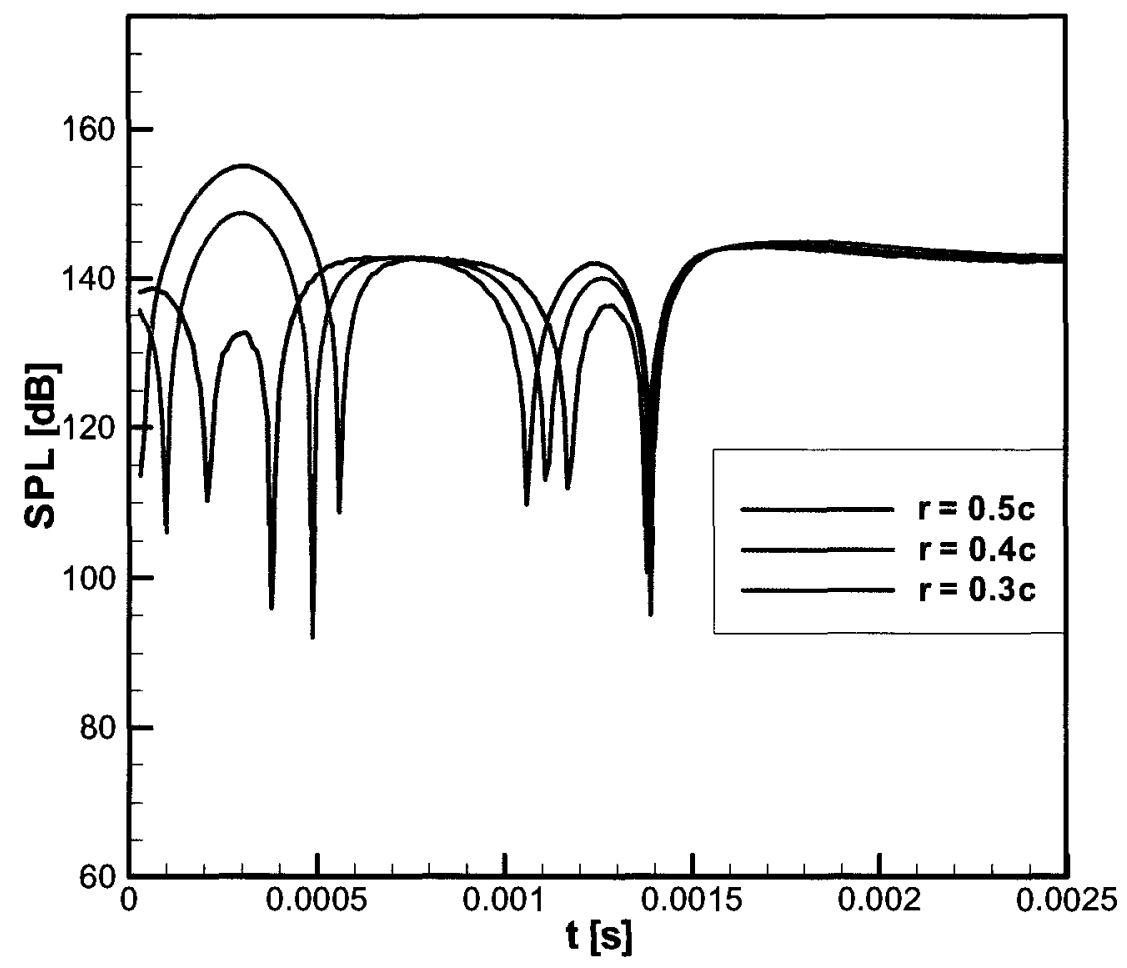

Figure 5.59 Comparison of SPL, $\alpha=\mathbf{0}^{0}, \mathbf{h}=-\mathbf{0 . 0 0} \mathbf{m}, \beta=15^{0}, \mathbf{x}=-\mathbf{0 . 1} \mathrm{m}$.

From Figure 5.57 and Figure 5.58 it can be seen that the SPL peaks decrease with the decrease of the vortex core size. The SPL presents a similar parabolic trend for all test cases, at the instant when the vortex encounters the airfoil, $t=0.0013 \mathrm{~s}$. The opening of the parabolic profile narrows as the vortex core size decreases, and this is due to the influence of vortex core radius. For a vortex with a large core radius, the induced velocity at the airfoil surface is sensed at an earlier stage of airfoil-vortex interaction, and this is reflected in the phase difference of SPL values, at about $t=0.0012 \mathrm{~s}$. As the vortex passes the airfoil leading edge, there is a perfect overlap of the SPL values for all test cases, as seen in Figure 5.57 and Figure 5.58, at the instant $\mathrm{t}=0.0014 \mathrm{~s}$, and this is a result of the vortex core distortion. Once 
the vortex core is distorted, there is no variation of the SPL values in time, the SPL reaching a constant value.

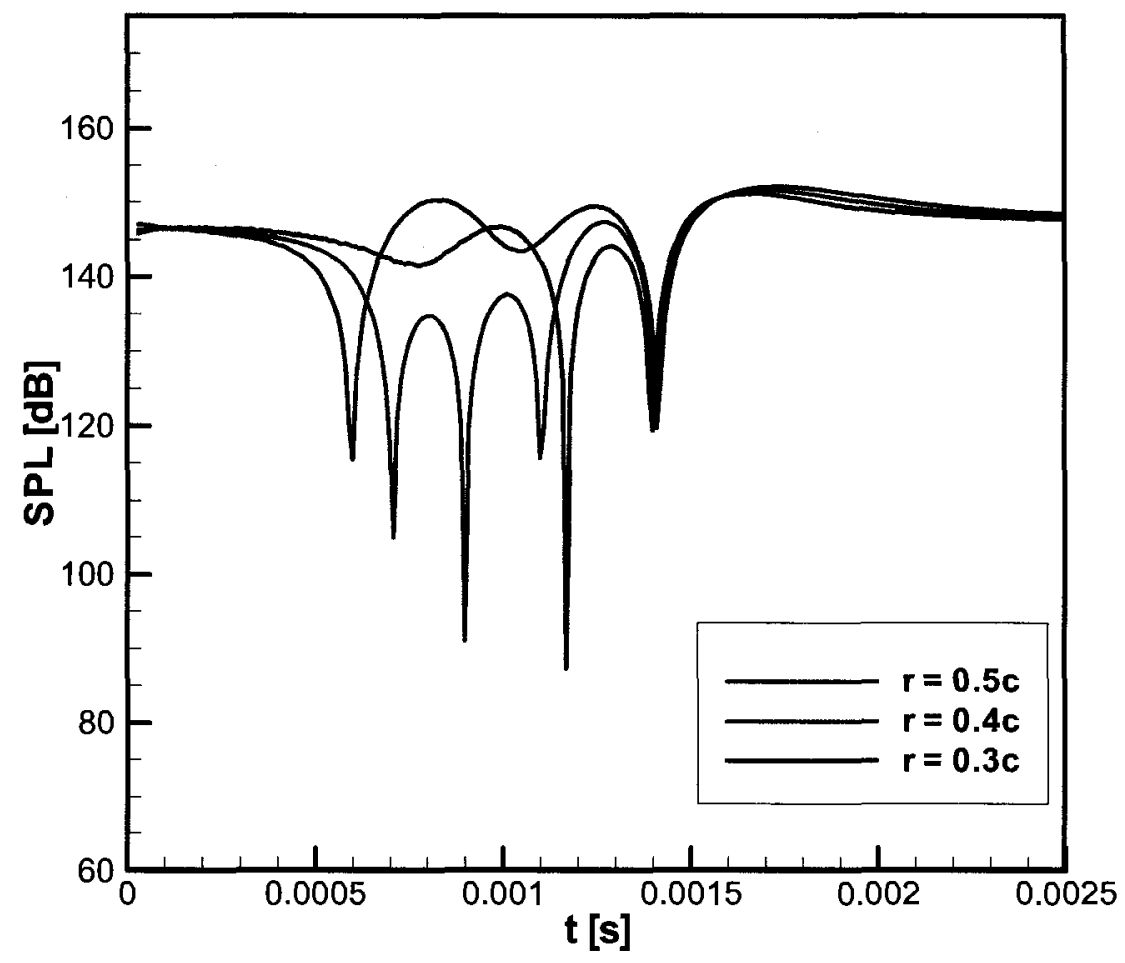

Figure 5.60 Comparison of SPL, $\alpha=0^{\circ}, \mathrm{h}=-\mathbf{0 . 0 0} \mathrm{m}, \beta=30^{\circ}, \mathrm{x}=-\mathbf{0 . 0 5 m}$. 


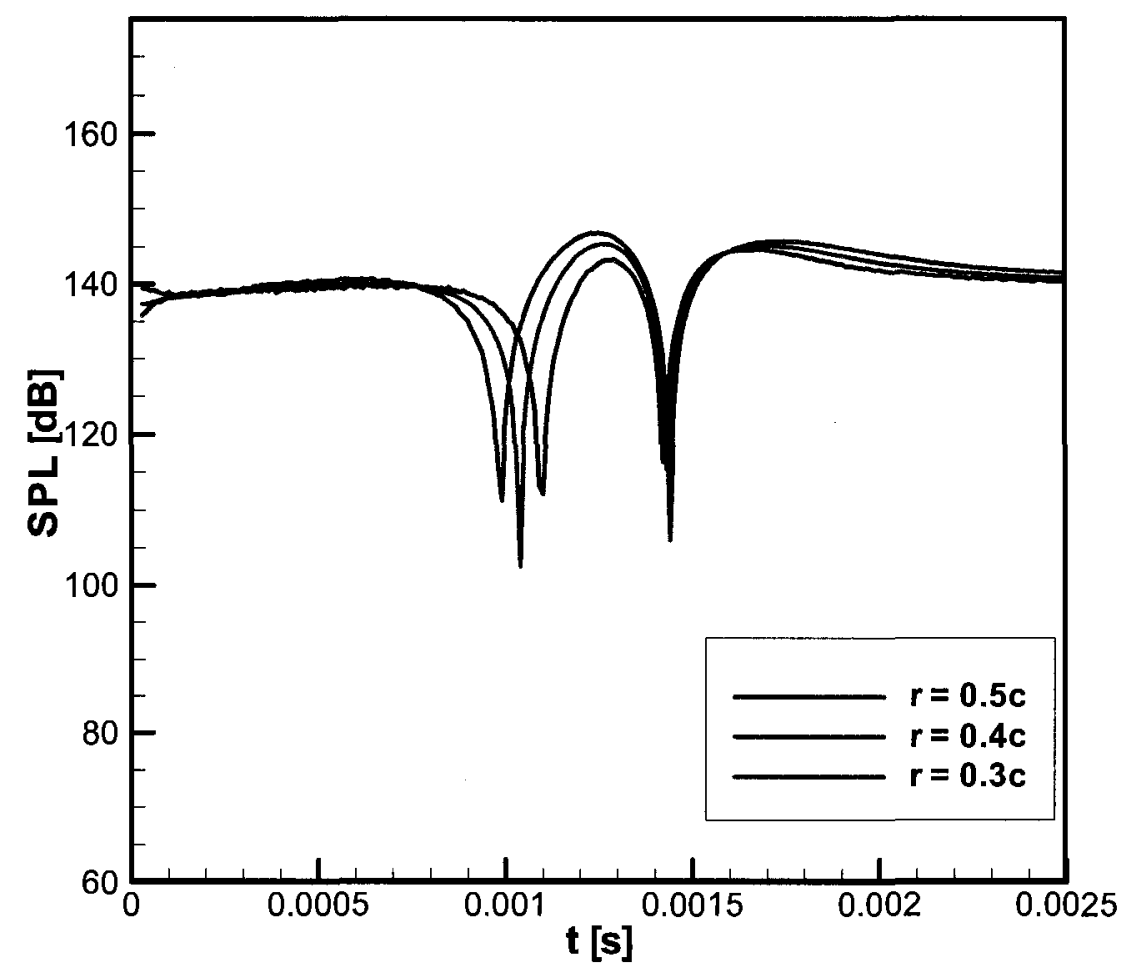

Figure 5.61 Comparison of SPL, $\alpha=\mathbf{0}^{\mathbf{0}}, \mathbf{h}=\mathbf{- 0 . 0 0} \mathbf{m}, \beta=30^{\circ}, \mathbf{x}=-\mathbf{0 . 1 m}$.

Similar behaviour of SPL was observed for a spanning angle $\beta=30^{\circ}$, as presented in Figure 5.59. From Figure 5.60 it can be seen that a point defined by $x=-0.1 \mathrm{~m}$ and spanning angle $\beta=30^{\circ}$ does not sense the vortex-generated acoustic noise since its location is far from the vortex core. 


\section{Chapter 6: Aerodynamic and Aeroelastic Investigations of Idealized Airfoil-Vortex Interaction}

\subsection{Aeroelastic Modeling}

Within the state of the art, the best possible aerodynamic models are obtained via computational fluid dynamics (CFD) models. By numerically solving the unsteady NavierStokes equations improved modeling of the flow and better understanding of fluid phenomena can be obtained.

While the structural dynamics can typically be well represented by a linear analysis it is generally agreed that the unsteady aerodynamic effects are extremely complex. At least some of the flow details such as shock motion, blade loading, viscosity and boundary conditions must be modelled to obtain realistic analyses.

Because very little data exists to isolate the most important of these details, the current state of the art utilises CFD analyses to capture as much of the physics as possible. When deriving an aeroelastic model we are often not concerned with the precise details of the flow field but instead with predicting certain relevant output quantities accurately. These outputs are typically the forces and moments acting on the blades and sometimes outgoing flow disturbances at the passage inlet and exit. The aerodynamic problem can therefore be viewed as an input-output system where blade motions, incoming flow perturbations and flow operating conditions provide the inputs. Similarly the structural model can be viewed as a means of obtaining the blade displacements and stresses given a specific forcing configuration. 
Figure 6.1 illustrates the concept of an input-output aeroelastic model. Computational models such as finite element models for the structure and CFD models for the flow should provide an accurate representation of the appropriate outputs given a set of input conditions.

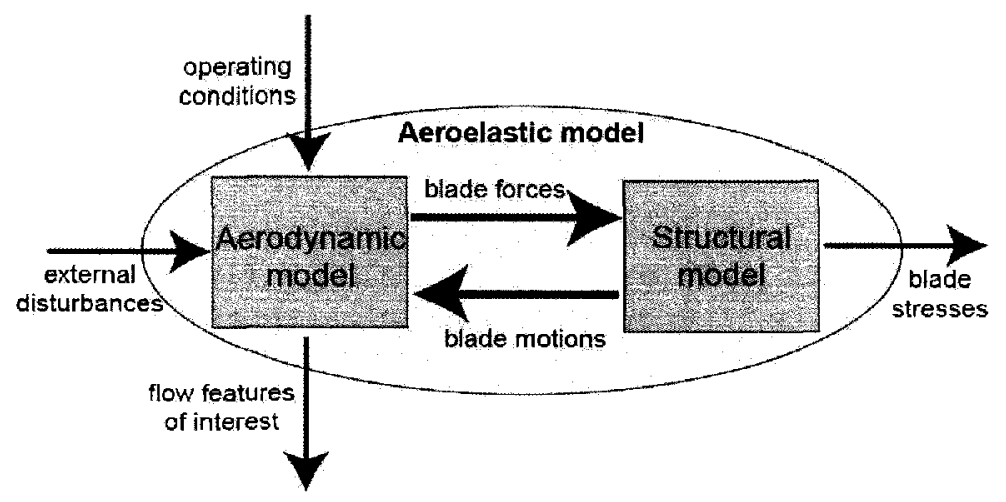

Figure 6.1 Input-output view of aeroelastic model.

Due to the complexity of the flow field such an analysis requires high fidelity computational fluid dynamics (CFD) analysis tools, due to the nonlinear behaviour of the aerodynamics and also high fidelity computational structural dynamics (CSD) analysis tools. One of the main issues of computational aeroelasticity related to BVI is the inherent numerical dissipation of CFD codes, which severely affects the preserving of the vortex characteristics. An alternative approach aiming at overcoming the issue associated with the numerical dissipation is largeeddy simulation (LES), which in spite of the high computational costs, preserves the characteristics of the vortex and provides the instantaneous values of the variables. Coupling of high fidelity CFD and CSD tools to solve aeroelastic problems has received interest only in the past few years. Huge computational power is required to make the use of such tools feasible. Continuous improvements in computer speed, memory, architecture and parallel processing have made solving these computationally intensive problems more cost efficient. 
Aeroelastic problems of aerospace vehicles are often dominated by flow nonlinearities and at times by large structural deformations. Therefore, coupled approaches are necessary to solve such problems accurately. Coupled approaches for solving aeroelastic problems are usually categorized in two ways: loosely or strongly coupled. The loosely coupled approaches can be integrated or modular. Integrated, loosely coupled methods alter the source code of either the CSD or CFD analysis tool by including the coupling schemes in either code. Though the codes are integrated, the CFD and CSD equations are not being altered and are solved independently. Modular, loosely coupled methods do not integrate the coupling schemes into either the CFD or CSD code. This allows the use of a variety of CFD/CSD codes. Strongly or fully (single domain) coupled approaches require the solution of the CFD and CSD equations simultaneously which necessitates the reformulation of the equations of each discipline.

In order to simplify the aeroelastic coupling problem, due to the highly turbulent flow, an idealized airfoil is the subject of investigation in the present work. In the present analysis, the aeroelastic response of an idealized-airfoil subject to blade-vortex interaction phenomenon is investigated using a CFD (Large-Eddy Simulation) based, strong coupling approach (two-way coupling). The idealized airfoil is modeled as a flat plate as schematically presented in Figure 6.2. The objective of the present work is to investigate the effect of vortex-idealized airfoil vertical miss-distance on the aeroelastic response of the idealized airfoil. The aeroelastic response of the idealized airfoil to AVI phenomenon is computed using a single degree of freedom (SDOF) mass/spring model as shown in Figure 6.3. It is obvious the fact that besides the flow field characteristics and structure properties and dimensions, the spring stiffness influences the aeroelastic response as well. The effect of the spring stiffness on the aeroelastic response of the flat plate is also subject of investigation 
in the present analysis. The aeroelastic response of the idealized airfoil is quantified by a comparison of aerodynamic coefficients for a rigid and elastic structure, respectively.

The objective of the current research is to investigate the effect of vertical missdistance on the aeroelastic response of an idealized airfoil using a CFD based, strong coupling approach (two way coupling).

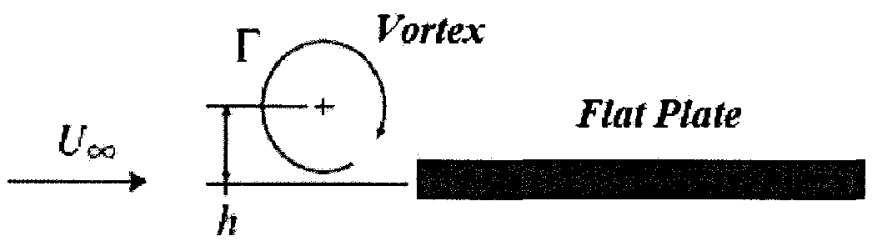

Figure 6.2 Blade-vortex miss-distance representation.

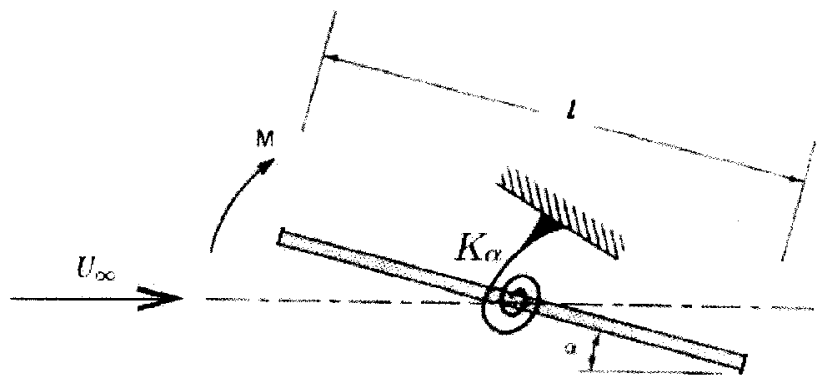

Figure 6.3 Schematic view of SDOF pitching idealized airfoil.

Computational aeroelasticity is a coupled fluid-structure problem which requires the exchange of forces and displacements between the flow and the structure, as well as the simultaneous solution of the CFD and CSD equations. In the general case, the boundary surfaces of the mesh used in the flow computation are different from those used in the 
structure computation. The aeroelastic coupling procedure requires only the grid point coordinates of the CFD and CSD grids to create the interface mappings.

The aeroelastic (CFD/CSD) coupling algorithm is presented in the following:

1. Obtain an intermediate or rigid steady state CFD solution

2. Calculate the pressures at the CFD grid points on the aerodynamic surface

3. Map pressures at the CFD grid points to forces on the CSD nodes

4. Obtain the structural response of the structure

5. Map displacements at the CSD nodes to the displacements on the CFD grid points of the aerodynamic surface

6. Deform the entire CFD grid

7. Repeat steps 1-6 until a pre-selected convergence criterion is met.

The aerodynamic pressures are calculated using the CFD code. The forces are calculated at each CFD grid point using the pressures and calculated areas. The forces at the CFD grid points of the structure are then mapped onto the CSD nodes. In a general analysis the CFD grid is much denser than the CSD grid.

In the present analysis a strongly or fully (single domain) coupled approach is used, requiring the solution of the CFD and CSD equations simultaneously which necessitates the reformulation of the equations of each discipline (CFD/CSD). The fluids are modeled using finite-volume method while the structures are modeled using finite element equations.

The mesh deformation strategy used in the present work is based on the spring analogy approach, which has been extensively used in the past, for aeroelastic calculations. In the spring analogy, the mesh is considered as fictitious springs. Boundary nodes are moved by aeroelastic computations and interior nodes are moved by the spring analogy with several 
iterations. Each edge of the mesh is represented by a spring whose stiffness is related to the length of the edge. The governing equations are closely related to a simple Laplace equation, as the displacements in each coordinate direction become decoupled and are governed by the equations:

$\left(\Delta x_{i}\right)_{m}=\frac{\sum_{j} k_{i j}\left(\left(x_{j}\right)_{m}^{n+1}-\left(x_{j}\right)_{m}^{n}\right)}{\sum_{j} k_{i j}} \quad m=1,2,3$

Batina proposed a grid movement method for unstructured grids where each edge in the computational grid is replaced by a linear spring whose stiffness is inversely proportional to the length of the edge. ${ }^{73}$

Thus, for the edge connecting node $i$ and $j$, the stiffness $k_{i j}$ of the spring is given by:

$k_{i j}=\frac{1}{\left(\left(x_{i}-x_{j}\right)^{2}+\left(y_{i}-y_{j}\right)^{2}\right)^{p / 2}}=\frac{1}{l_{i j}^{p}}$

where $\left(x_{i}, y_{i}\right)$ and $\left(x_{j}, y_{j}\right)$ are the coordinates of nodes $i$ and $j$ and $p$ is a predetermined coefficient, usually 1 or 2 and $l_{i j}=p\left(\left(x_{i}-x_{j}\right)^{2}+\left(y_{i}-y_{j}\right)^{2}\right)^{p / 2}$.

Given a set of nodal displacements or forces acting on the boundary of the computational grid, the following equations for the interior displacements are solved iteratively until all the forces are in equilibrium. The governing equation of motion for the structure under aerodynamic loading can be written in the form:

$[M]\left[\delta^{\prime \prime}\right]+[C]\left[\delta^{\prime}\right]+[K][\delta]=\{F(t)\}$

where $[\mathrm{M}],[\mathrm{C}]$ and $[\mathrm{K}]$ represent the mass matrix, damping matrix and stiffness matrix respectively. $\{F(\mathrm{t})\}$ is the aerodynamic loading and $\delta$ is the displacement. 


\subsection{Aerodynamic and Aeroelastic Results}

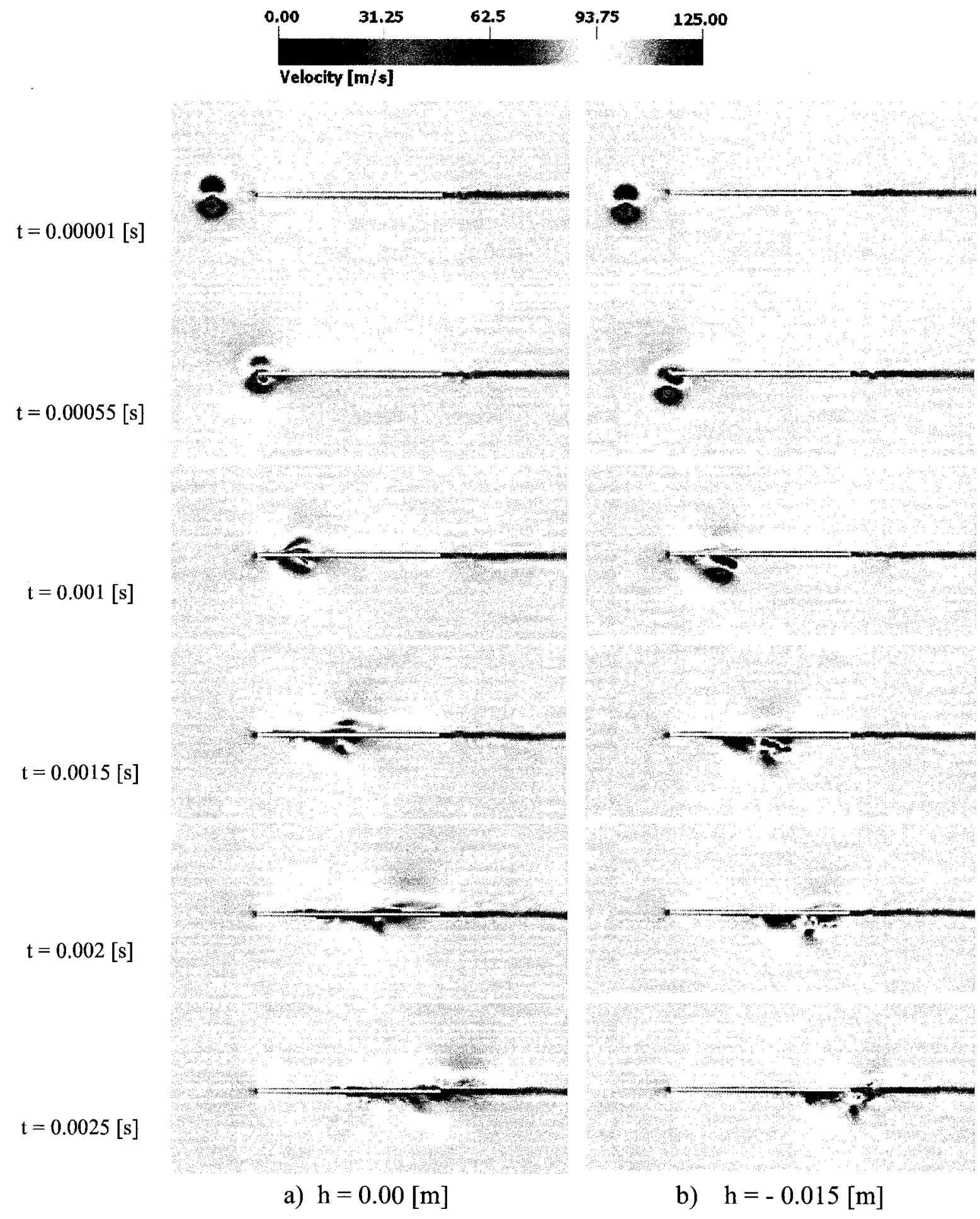

Figure 6.4 Time evolved fluid structures (rigid idealized airfoil), magnitude velocity. 
Figure 6.4 shows the LES results of instantaneous magnitude of velocities at six different instants in time for two out of the four test case, respectively $h=0.00 \mathrm{~m}$ and $\mathrm{h}=-0.015 \mathrm{~m}$. For all test cases, the idealized airfoil senses an induced velocity from the vortex, pointed downward. From Figure 6.4, it is apparent that the vortex core is almost destroyed as the vortex interacts directly with the idealized airfoil $(\mathrm{h}=0.00 \mathrm{~m})$, and there are wake perturbations due to the presence of the remaining vortex in the flow field. Also it can be seen that as the vortex approaches the idealized airfoil and travels the chord length of it, the velocity distribution on the upper and lower surface changes continuously until the vortex leaves the idealized airfoil. However for test case $2, \mathrm{~h}=-0.015 \mathrm{~m}$, the lower surface of the airfoil sees a higher velocity as the vortex encounters the airfoil.

The change in velocity distribution field influences the pressure distribution around the idealized airfoil and causes fluctuations of the stagnation point along the upper and lower surface of the airfoil.

From Figure 6.4, for the two test cases $h=0.00 \mathrm{~m}$ and $\mathrm{h}=-0.015 \mathrm{~m}$, it can be seen that a small change in the vertical miss-distance has a great impact on the vortex-idealized airfoil mechanism of interaction. The vortex-idealized airfoil mechanism of interaction has impact on both velocity and pressure field and as one expects; it influences the aeroacoustic field as well. From Figure $6.4 \mathrm{~b}$, it can be seen that when the vortex is in the neighborhood of airfoil passing underneath of airfoil $(t=0.0025 \mathrm{~s})$, due to the mechanism of interaction, the vortex is highly distorted and this disturbs the near region flow field. It can be noticed that at the instants when the remaining part of the distorted vortex leaves the idealized airfoil there are wake perturbations. As the remaining part of the vortex travel farther downstream, the flow around the idealized airfoil remains undisturbed and the lift coefficient converges to a 
zero value. As the vertical miss-distance increases, the variations of velocity field on the upper and lower surface are less significant due to the fact that the interaction between vortex and airfoil is less severe and hence the vortex preserves better its characteristics. 


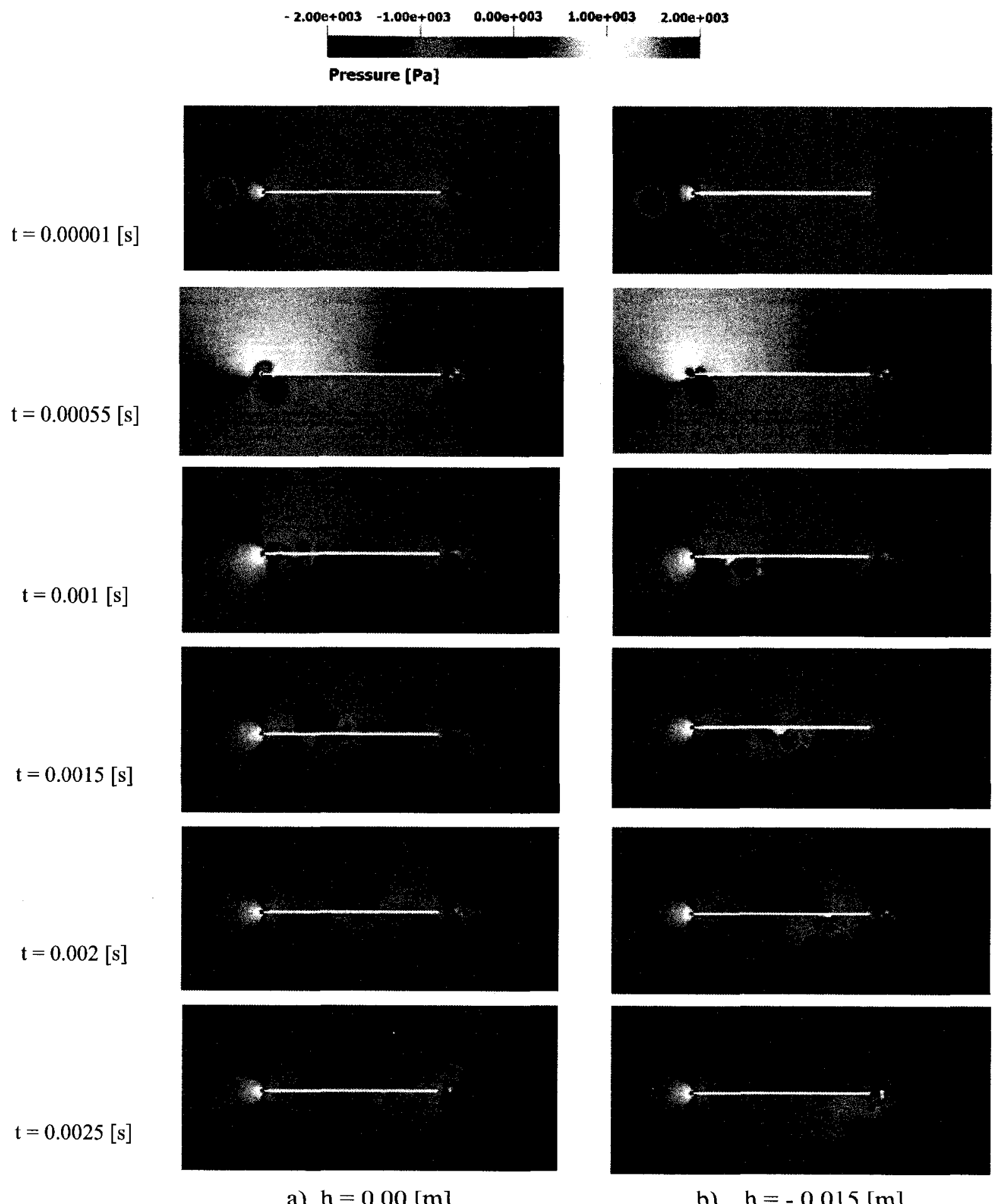

$\begin{array}{ll}\text { a) } \mathrm{h}=0.00[\mathrm{~m}] & \text { b) } \mathrm{h}=-0.015[\mathrm{~m}]\end{array}$

Figure 6.5 Time evolved fluid structures (rigid idealized airfoil), magnitude pressure. 
Figure 6.5 presents the time-varying pressure field for the case of an idealized airfoil (flatplate) interacting with oncoming vortex. Opposite to the case of NACA0012 airfoil, the pressure field presents a different behaviour especially at the instant and after the instant of interaction. Due to the geometry of the flat plate (thin rigid structure) the vortex is completely destroyed, for the case of vertical miss-distance $h=0.00 \mathrm{~m}$ as seen at the instant $t=0.001 \mathrm{~s}$. Similar to the case of NACA 0012 airfoil, as the vertical miss-distance increases, the interactions become less severe and the vortex preserves better its characteristics, as seen in Figure 6.5, at instant $\mathrm{t}=0.001 \mathrm{~s}$.

Figure 6.6 presents the time-varying SPL, for two vertical miss-distances. Due to the fact that the vortex-idealized airfoil interactions are severe and the vortex is completely destroyed, the SPL present large variations describing undefined contours of constant value $(100 \mathrm{~dB})$. 


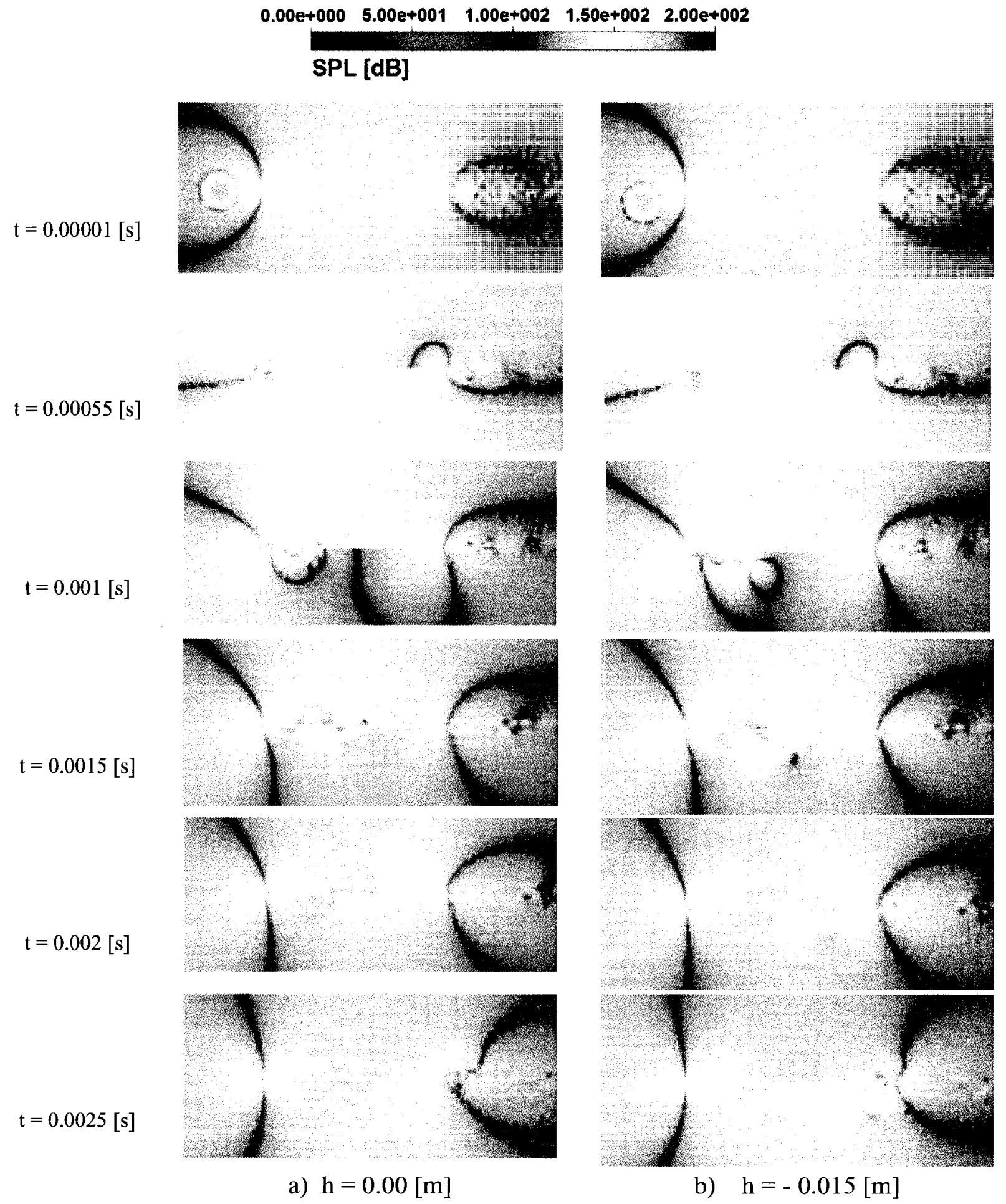

Figure 6.6 Time history of SPL, for an idealized airfoil. 


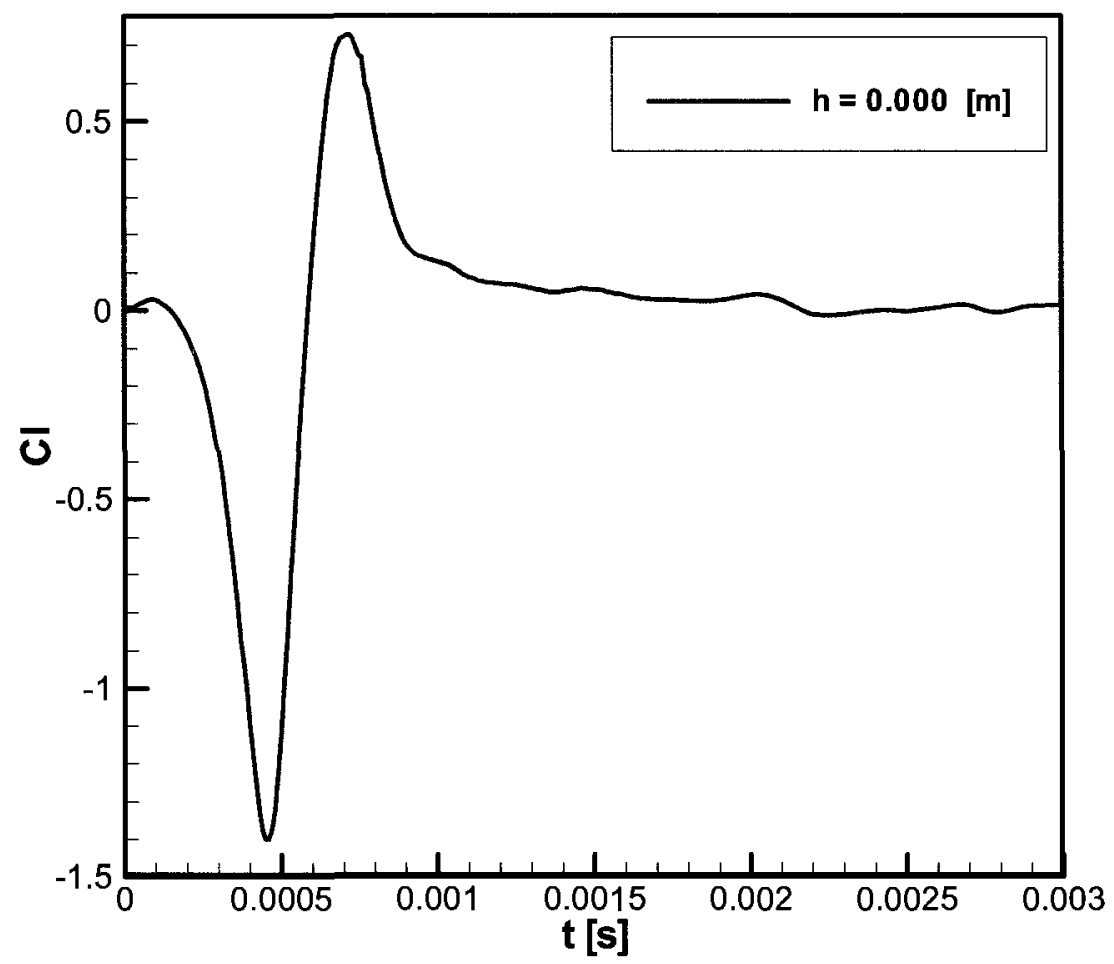

Figure 6.7 Time history of lift coefficient (rigid structure).

Figure 6.7 presents the temporal variation of lift coefficient for a vortex superimposed on a uniform flow encountering a rigid idealized airfoil (thin flat plate) at a zero angle of attack and a vertical miss-distance $\mathrm{h}=0.00 \mathrm{~m}$. The lift coefficient is influenced by the pressure distribution over the idealized airfoil surface. From Figure 6.7, it can be seen that there is a continuous decrease in lift as the vortex approaches the airfoil (until a minimum value of lift is achieved), followed by a sudden jump as the vortex core passes the leading edge. This sudden jump in lift coefficient is generated by a sudden change in the pressure coefficient. Although there is an increase of lift coefficient value as the vortex passes the leading edge, the absolute value of this maximum is smaller than the absolute minimum value. This difference in minimum-maximum absolute values is due to the vortex core distortion when 
the vortex encounters the idealized airfoil. When the remaining part of the distorted vortex is far downstream from the idealized airfoil, the lift coefficient converges asymptotically to a zero value and the flow field is almost undisturbed.

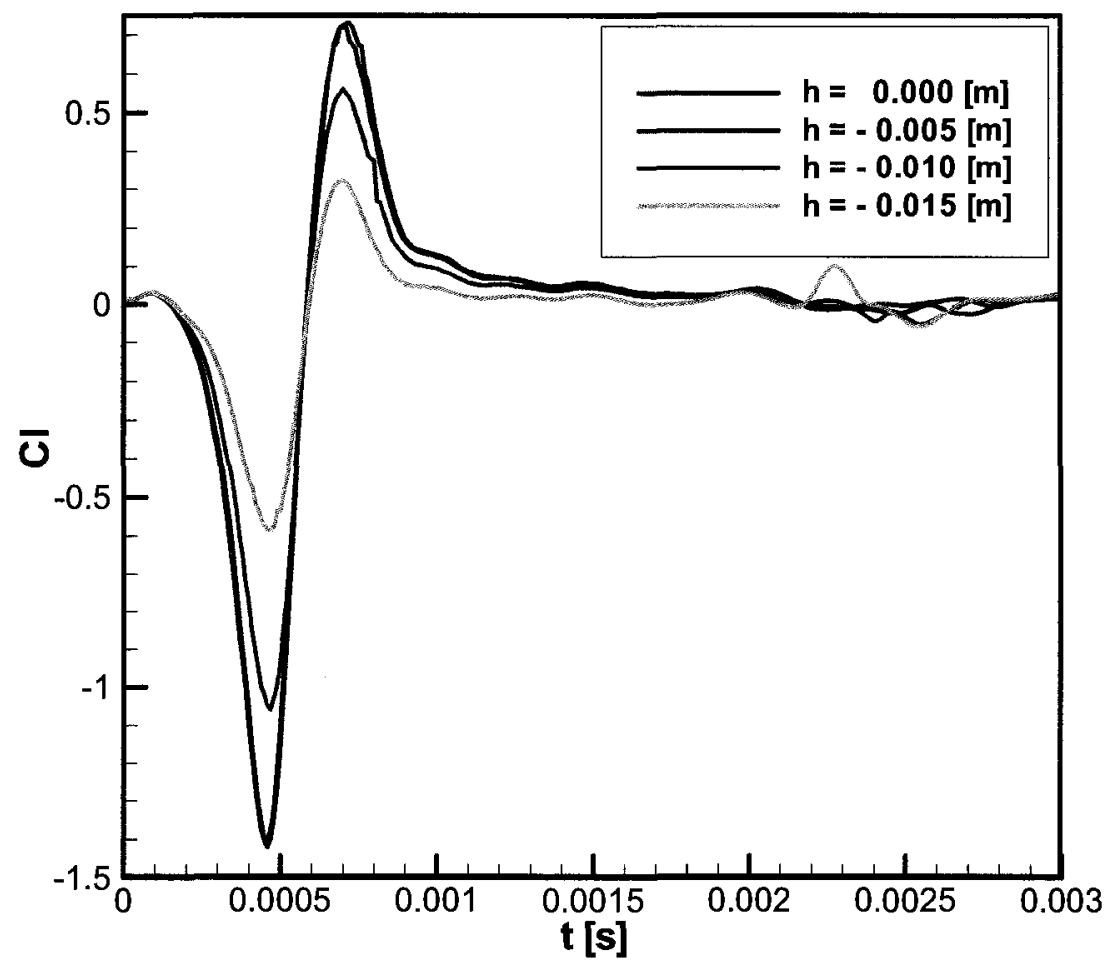

Figure 6.8 Comparison of lift coefficient (rigid structure).

Similar behaviour of lift coefficient was observed for the other three test cases $h=-0.005 \mathrm{~m}$, $\mathrm{h}=-0.01 \mathrm{~m}$ and $\mathrm{h}=-0.015 \mathrm{~m}$. Therefore a comparison of time-varying lift coefficient for all four test cases would be more appropriate rather than a detailed description of each test case alone. In this sense, Figure 6.8 presents a comparison of lift coefficient for four different vortex-idealized airfoil vertical miss-distances $h=0.00 \mathrm{~m}, \mathrm{~h}=-0.005 \mathrm{~m}, \mathrm{~h}=-0.01 \mathrm{~m}$ and $\mathrm{h}=$ $0.015 \mathrm{~m}$. 
For all test cases, the lift coefficient presents a sudden jump at the time when the vortex encounters the idealized airfoil $(\mathrm{t}=0.0005 \mathrm{~s})$. This sudden jump decreases as the airfoilvortex vertical miss-distance increases. This decrease is due to the fact that the vortexidealized airfoil interactions are less significant and have less impact on the flow field. From the comparison of lift coefficient it can be seen that with the increase of vortex-idealized airfoil vertical miss-distance, the jump in lift coefficient at $\mathrm{t}=0.0005 \mathrm{~s}$ tends to become more symmetric with respect to a zero value and the transition from negative to positive values is smoother.

Hence, the jump of lift coefficient, for vertical miss-distance $h=-0.015 \mathrm{~m}$, at instant $t$ $=0.0005 \mathrm{~s}$ becomes almost symmetric respect to a zero value and this due to the fact that the vortex-idealized airfoil mechanism of interaction is less severe and hence the vortex remains almost undisturbed having enough strength to disturb the flow field around the airfoil in a particular manner. As the vertical miss-distance increases fluctuations of lift coefficient can be observed at the instant when the vortex leaves the idealized airfoil $(t=0.0023 \mathrm{~s})$.

As it was already mentioned, the present analysis focuses on the aeroelastic response of the idealized airfoil, undergoing a BVI phenomenon. Consequently, similar analysis is performed for the case of a flexible structure. The aeroelastic response of the idealized airfoil to AVI phenomenon is computed using a single degree of freedom (SDOF) mass/spring model as shown in Figure 6.2. The idealized airfoil has the freedom to oscillate about the elastic axis in a vertical plane. The aeroelastic response of the idealized airfoil is quantified by a comparison of lift coefficient, for the case of a rigid and respectively flexible structure. 


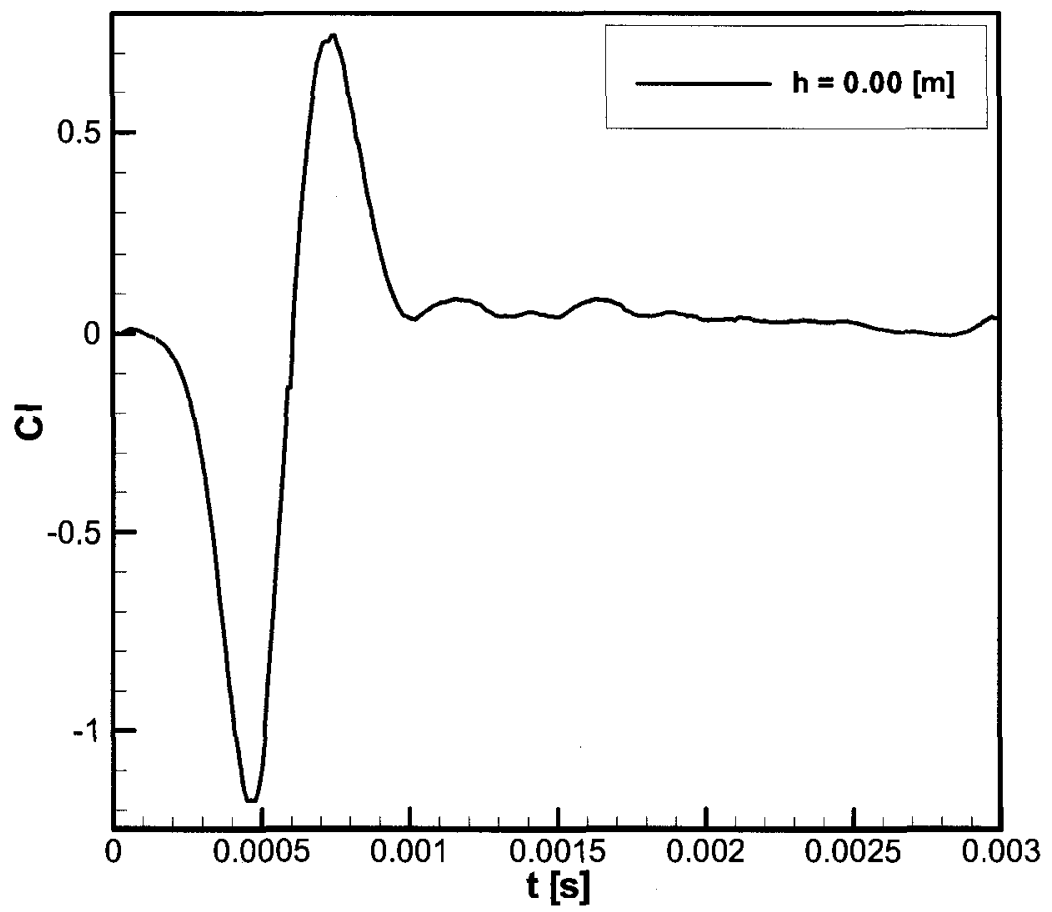

Figure 6.9 Time history of lift coefficient (flexible structure).

Figure 6.9 shows the lift coefficient for the case of the flexible structure undergoing AVI phenomenon for a vertical miss-distance $\mathrm{h}=0.00 \mathrm{~m}$. It is important to emphasize the fact that the geometry of the idealized airfoil and the computational domain are identical with the previous case of rigid idealized airfoil.

Similar to the case of a rigid structure, the lift coefficient presents the similar trend for the case when the structure is flexible. It is worth to notice that there are fluctuations in the lift coefficient as the vortex travels the chord length of the idealized airfoil and they are a result of the aeroelastic response to AVI. 


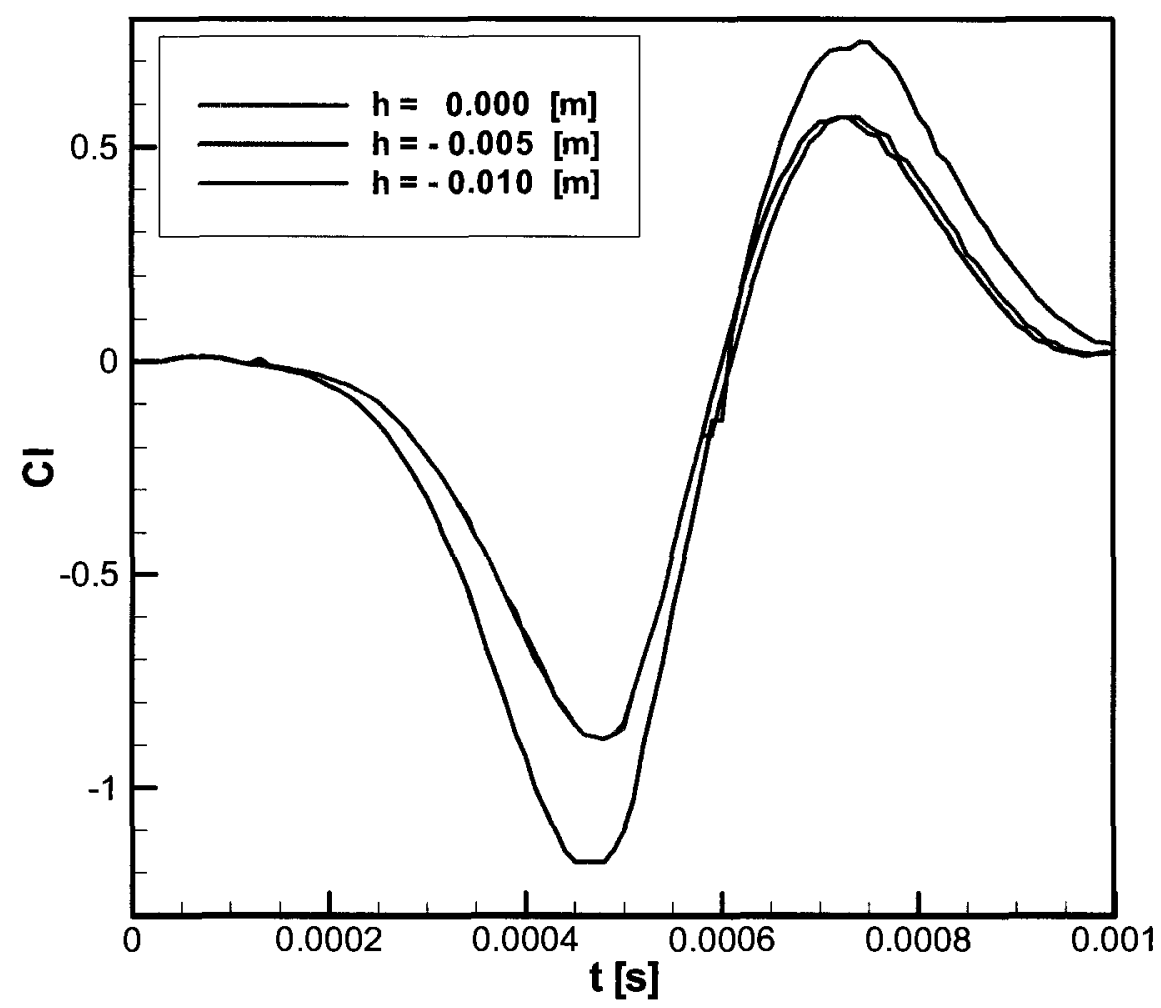

Figure 6.10 Lift coefficient comparison (flexible structure).

Figure 6.10 presents the comparison of lift coefficient for three different test cases for a flexible structure. Similar to the case of a rigid structure, the peaks of lift coefficient decrease as the vertical miss-distance increases. However, there are differences in the lift coefficients trend between rigid and flexible structure. It is important to notice that the lift coefficient for a rigid structure presents almost identical values for vertical miss-distances $h=0.00 \mathrm{~m}$ and $\mathrm{h}$ $=-0.005 \mathrm{~m}$, respectively, as seen in Figure 6.10 . For the case of a flexible structure this similarity of lift coefficients is not present anymore, for the vertical miss-distances $h=0.00 \mathrm{~m}$ and $\mathrm{h}=-0.005 \mathrm{~m}$, as seen in Figure 6.10. Instead, the values of lift coefficient are almost identical for the vertical miss-distances $h=0.00 \mathrm{~m}$ and respectively $h=-0.005 \mathrm{~m}$, Figure 6.10 . 
These differences and similarities of lift coefficient are the direct result of the aeroelastic response of the idealized airfoil to AVI phenomenon.

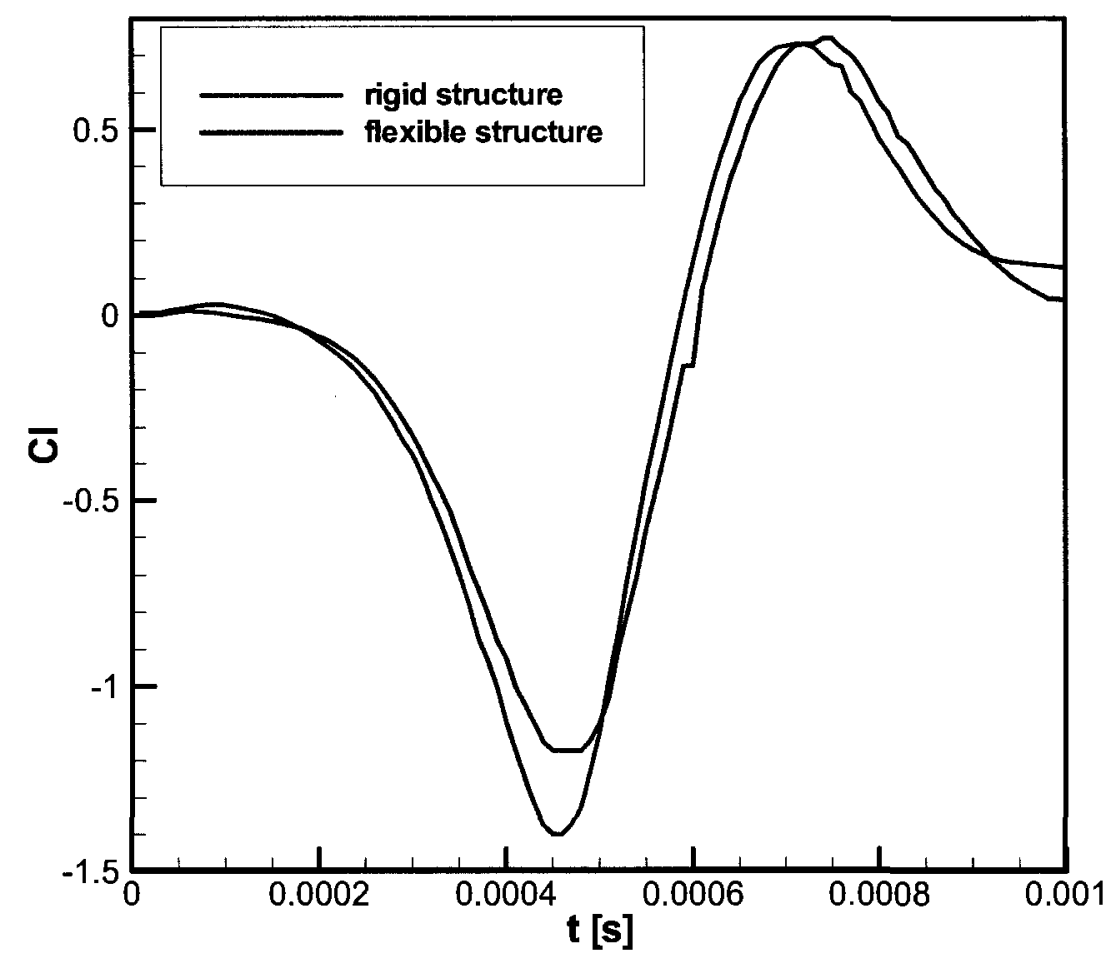

Figure 6.11 Lift coefficient comparison, vertical miss-distance $h=0.00 \mathrm{~m}$.

For a better understanding of the aeroelastic phenomenon, the aeroelastic response of the idealized airfoil is quantified by a comparison of lift coefficient, for the case of a rigid and respectively flexible structure, as presented in Figure 6.11. The results from the present investigation show that there is an aeroelastic response of the idealized airfoil to BVI phenomenon, influencing the aerodynamic coefficients. The aeroelastic response is exerted through a phase difference as a delayed response to AVI and a minimization of vortex impact on the lift coefficient, as seen in Figure 6.11. 


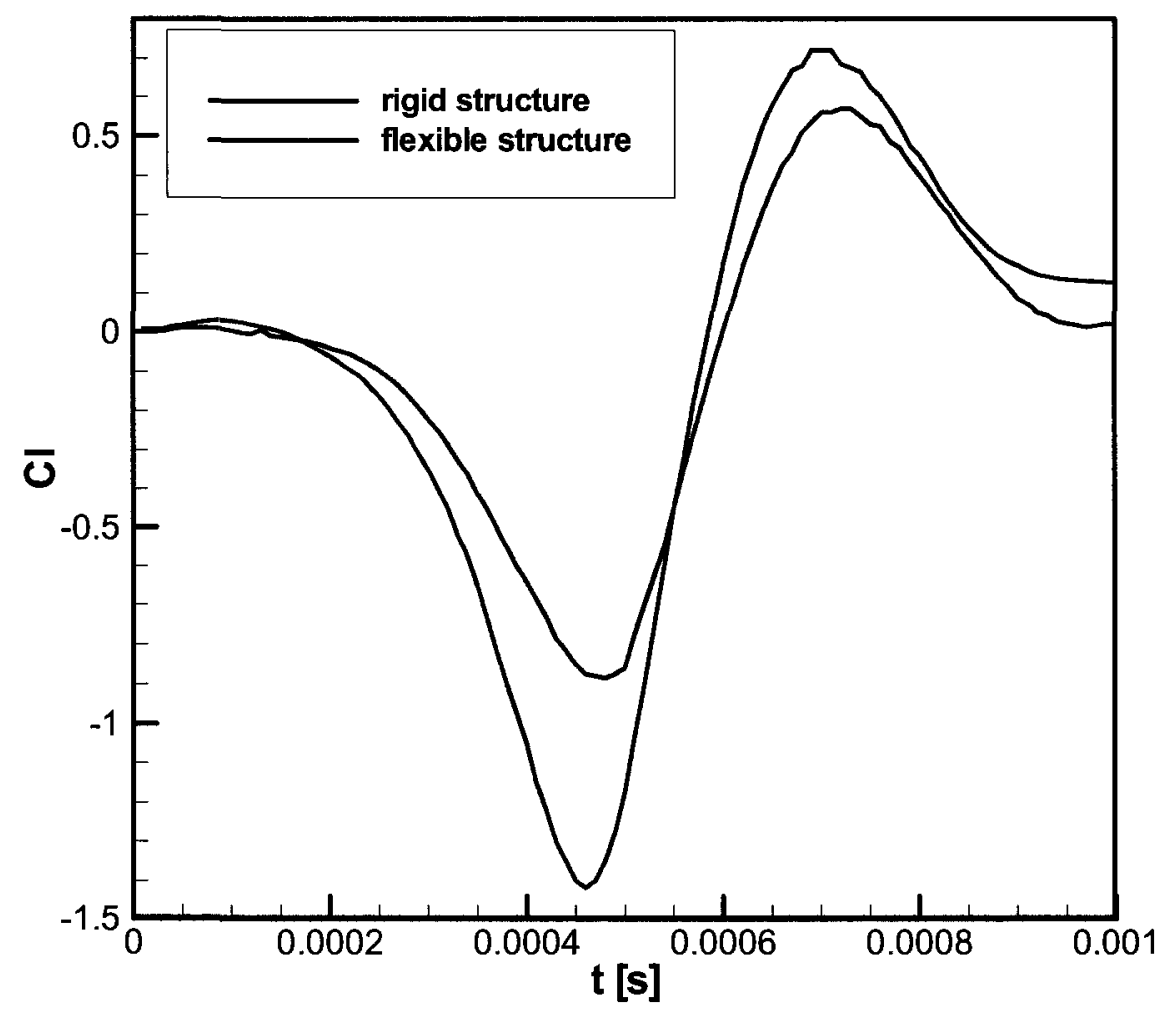

Figure 6.12 Lift coefficient comparison, vertical miss-distance $h=-\mathbf{0 . 0 0 5 m}$.

Figure 6.12 presents the comparison of lift coefficient for the case of a flexible and rigid structure for a vertical miss-distance $h=-0.005 \mathrm{~m}$. From Figure 6.12 it can be seen that with the increase of vertical miss-distance, the differences of lift coefficient for the two test cases are more evident and this is due to the influence of vertical miss-distance on the mechanism of interaction.

From Figures 6.11 and 6.12 it can be seen that independent of vertical miss-distance the amplitudes of lift coefficient are smaller for the case of flexible structure. The fact that the differences in lift coefficient for rigid and flexible structure are not very large is due to the spring stiffness. It is well known that the flow speed and spring stiffness influences the 
aeroelastic response of a SDOF idealized airfoil. If the flow speed is low or the spring stiffness is large, the aeroelastic response is small.

The smaller peak values of lift coefficient for the case of a flexible structure suggest that the interaction is less severe when compared with the case of a rigid structure. The flexible nature of the idealized airfoil minimizes the impact of the vortex on the lift coefficient, acting as a dumper. 


\section{Chapter 7: Conclusions}

Parametric investigations of flow past circular cylinder were conducted to asses the applicability of large-eddy Simulation to numerical investigation of vortex dominated flows. In this sense investigations based on the vortex-cylinder horizontal miss-distance were conducted, using both URANS and LES and a comparison of the two approaches was performed. The results turned that URANS is a dissipative approach and its use for the simulation of vortex dominated flows and aeroacoustic investigations remains limited. From the aerodynamic analysis it was observed that the presence of the vortex in the flow field and the vortex-cylinder mechanism of interaction influences the von Kármán vortex street and Strouhal number. An increase of Strouhal number due to the presence of the vortex in the flow field was observed. Also the present investigation revealed the fact that the instant of interaction between vortex and cylinder plays a key role in the development of the flow field in the wake of cylinder. Based on the information collected from the investigation of flow past cylinder a parametric analysis was conducted for the case of NACA0012 airfoil.

The present analysis focused on the parametric investigation of airfoil-vortex mechanism of interaction. The vortex was released upstream of airfoil and the unsteady flow field results were stored at regular time intervals, corresponding to different vortex core locations along the vortex trajectory and were used for calculating the aerodynamic coefficients.

Calculations were made for different BVI problems with varying the airfoil-vortex offset distance, angle of attack and vortex characteristics. The presence of the vortex in the flow field induces a velocity at the surface of airfoil and generates a time-varying lift effect 
and fluctuations of sound pressure level. The variation of lift coefficient depends on the airfoil-vortex vertical miss-distance, angle of attack, vortex characteristics and implicitly on the airfoil-vortex mechanism of interaction. The airfoil-vortex mechanism of interaction is associated with flow separation and reverse flow indicated by the negative values of drag coefficient. The airfoil-vortex mechanism of interaction at small vertical miss-distances generates reverse flow. As the airfoil-vortex vertical miss-distance increases no flow separation is observed. A decrease of the aerodynamic coefficients was observed with the increase of airfoil-vortex vertical miss-distance, angle of attack. However, an increase of aerodynamic coefficients was observed with the increase of vortex core size.

A decrease of SPL with the increase of spanning angle, measured from the rotor disc plane was observed as well. The most significant variations in both aerodynamic coefficients and sound pressure level were observed for small values of airfoil-vortex vertical missdistance. The interrogation points located far from the leading edge are the first ones sensing the vortex-generated acoustic noise, while the interrogation points located closer to the leading edge are the ones that sense the vortex-generated acoustic noise at a later time. The interrogation points located in a horizontal distance less than the diameter of the vortex core sense the combined effect of both vortex-generated acoustic noise and BVI noise. It was observed that the vortex-generated acoustic noise is most sensed by the points located in the proximity of the vortex travel path.

From the present analysis it can be concluded that the airfoil-vortex vertical missdistance, angle of attack, vortex characteristics and implicitly the airfoil-vortex mechanism of interaction influences the acoustic field in a specific manner. 
The aeroelastic analysis shows that there is an aeroelastic response of the idealized airfoil to the interaction phenomenon, affecting the aerodynamic coefficients. The flexible nature of the idealized airfoil minimizes the impact of the vortex on the aerodynamic coefficients. In this sense a decrease of lift peaks at the instant of interaction was observed. The flow velocity and the spring stiffness constant are important parameters that affect the mechanism of interaction.

Although relatively expensive from a computational point of view, LES can simulate fluid structures that cannot be captured by traditional methods and hence it is the suitable and affordable tool to be used in the prediction of turbulent flows. 


\section{Chapter 8: $\quad$ Future work}

The present aerodynamic, aeroacoustic and aeroelastic analysis opens the horizon for a wide range of investigations that can be conducted, especially regarding the aeroelastic investigations. In this sense a potential analysis would be the numerical investigation of the aeroelastic effect of a deformable airfoil leading edge on the AVI/BVI mechanism of interaction and aerodynamic coefficients. A control of the leading edge of airfoil might provide a decay of airfoil-vortex interactions and minimizing its effect on the aerodynamic coefficients and aeroacoustic field. A potential solution to this problem is suggested in the Figures 8.1. and 8.2.

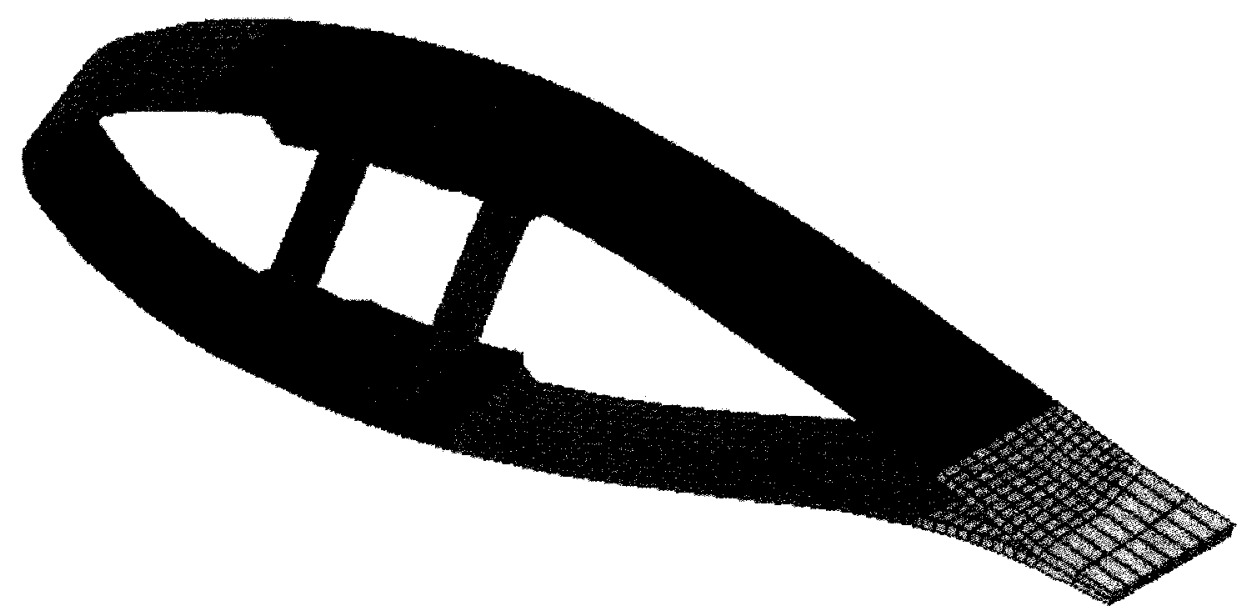

Figure 8.1 Multi beam-like structure model. 


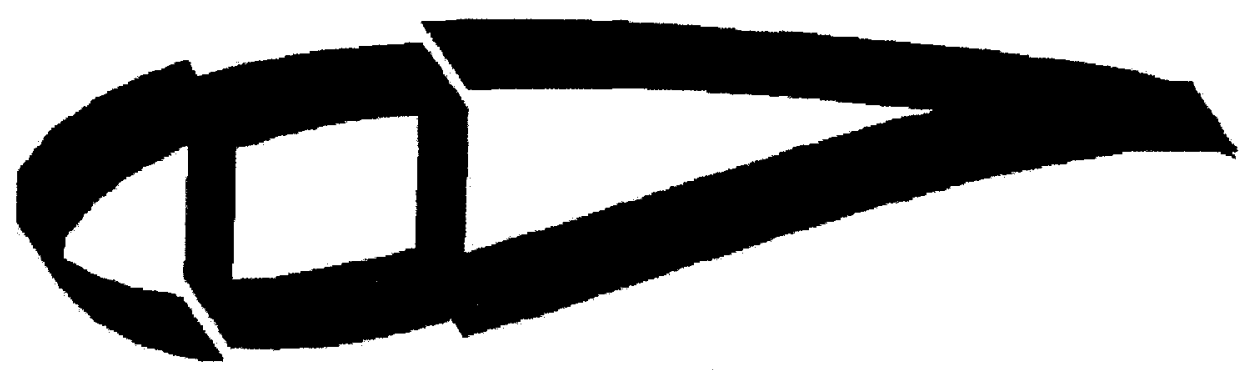

Figure 8.2 Airfoil multi beam-like structure model.

The proposed model consists of a multi beam-like structure where the airfoil surface has the freedom to deform under the action of aerodynamic forces. The two-way coupling aeroelastic approach developed in the present work for the case of an idealized-airfoil; ease the way toward the solution for a multi beam-like structure.

Although challenging, the suggested future work might bring a significant contribution to the ongoing research in the rotorcraft community regarding the reduction of helicopter noise and vibrations. 


\section{References}

${ }^{1}$ Spalart, P. R., Airplane trailing vortices, Annual Revue Fluid Mechanics, Vol.30, pp. 107$138,1998$.

${ }^{2}$ Opoku, D., Nitzsche, F., Acoustic validation of a new code using particle wake aerodynamics and geometrically-exact beam structural dynamics, The Aeronautical Journal of the UK Royal Aeronautical Society, 2005.

${ }^{3}$ Tangler, J. L., Experimental Investigation of the Subwing Tip and Its Vortex Structure, NASA CR-3058, Nov., 1978.

${ }^{4}$ Smith, D. E., and Sigl, D., "Helicopter Rotor Tip Shapes for Reduced Blade Vortex Interaction - An Experimental Investigation," AIAA Paper 95-0192, Jan. 1995.

${ }^{5}$ Martin, P. B., and Leishman, J. G., "Trailing Vortex Measurements in the Wake of a Hovering Rotor Blade with Various Tip Shapes," 58-th AHS, Annual Forum, Montreal, Canada, June, 2002.

${ }^{6}$ Hoad, D. R., "Helicopter Model Scale Results of Blade-Vortex Interaction Impulsive Noise as Affected by Tip Modification," Proceedings of the 36th American Helicopter Society Annual Forum, Washington, DC, May 13-15, pp.80.62.1-80.62.13, 1980.

${ }^{7}$ Coyne, A. J., Bhagwat, M. J., and Leishman, J.G., Investigation into the Rollup and Diffusion of Rotor Tip Vortices using Laser Doppler Velocimetry, Proceedings of the 53rd AHS Annual Forum, Virginia Beach, VA, Apr. 29-May 1, 1997.

${ }^{8}$ Wong, O. D., Formation and Evolution of Tip Vortices of an Isolated Rotor in Forward Flight, Ph.D. Dissertation, School of Aerospace Engineering, Georgia Institute of Technology, December, 2001.

${ }^{9}$ Mahalingam, R., Komerath, N. M., "Measurements of the Near Wake of a Rotor in Forward Flight," AIAA Paper 98-0692, January, 1998.

${ }^{10}$ Mahalingam, R., Structure of the Near Wake of a Rotor in Forward Flight and its Effect on Surface Interactions, Ph.D. Dissertation, School of Aerospace Engineering, Georgia Institute of Technology, June, 1999.

${ }^{11}$ Schlichting, H., Boundary Layer Theory, Pergamon Press Ltd, 1955.

${ }^{12}$ Dacles-Mariani, J., Kwak, D., and Zilliac, G., On Numerical Errors and Turbulence Modeling in Tip Vortex Flow Prediction, Int. J. Numer. Meth. Fluids, Vol. 30, pp. 65-82, 1999. 
${ }^{13}$ Hardin, J. C., Lamkin, S. L., Concepts for Reduction of Blade/Vortex Interaction Noise, J.Aircraft, vol.24, no.2, 1997.

${ }^{14}$ Yu, H. Y., 2000, Rotor blade-vortex interaction noise, Progress in Aerospace Sciences, vol. 36, pp. 97-115, 2002.

${ }^{15}$ McAlister, K., Measurements in the Near Wake of a Hovering Rotor, AIAA 96-1958, Fluid Dynamics Conference, June 1996.

${ }^{16}$ White, R. P. Jr., Balcerak, J. C., Pegg, R. J., Summary of Results Indicating the Beneficial Effects of Rotor Vortex Modification, Proceedings of the National Symposium on Helicopter Aerodynamic Efficiency, Hartford, Conn., March 6, 7, pp. 5.1-5.15, 1975.

${ }^{17}$ Gowanlock, D. K., Matthewson, C. S., Control of Rotor Tip Vortices, The 37-th AIAA Aerospace Sciences Meeting and Exhibit, Reno, NV, Jan 11-14, 1999.

${ }^{18}$ Han, Y. O., Leishman, J. G., Investigation of Helicopter Rotor-Blade-Tip-Vortex Allevation Using a Slotted Tip, Proceedings of the 59-th AHS, Annual Forum, Phoenix, AZ, May 7-10, 2003.

${ }^{19}$ Han, Y. O., Leishman, J. G., Investigation of Helicopter Rotor-Blade-Tip-Vortex Allevation Using a Slotted Tip, AIAA Journal, Vol. 42, No.3, March, 2004.

${ }^{20}$ Cesnik, C., Shin, S., On the modeling of integrally actuated helicopter blades, Int.J.of Solids and Struct.,38:1765-1789, 2001.

${ }^{21}$ Cesnik, C., Shin, S., On the Twist Performance of a multiple-cell active helicopter blade, J.of Smart Materials and Structures, 10:53-61, 2001.

${ }^{22}$ Smagorrinsky, J. S., General circulation experiments with the primitive equation, Monthly Weather Rev.91:99-164, 1963

${ }^{23}$ Menter, F. R., Two-Equation Eddy-Viscosity Turbulence Models for Engineering Applications, AIAA Journal, Vol.32, No.8, pp. 1598-1605, 1994.

${ }^{24}$ Menter, F. R., Zonal Two-Equation k- $\omega$ Turbulence Models for Aerodynamic Flows, AIAA Paper 93-2906, 1993.

${ }^{25}$ Germano, M., Piomelli, U., Moin, P., W. H. Cabot, A dynamic subgrid-scale eddy viscosity model. Phys. Fluids A, 3(7):1760-1765, 1991.

${ }^{26}$ Lilly, D. K., On the application on the eddy viscosity concept in the inertial sub-range of turbulence, NCAR Manuscript, pp195-200, 1996. 
${ }^{27}$ Papadakis, M., Nizampatnam L., Hoffmann, K., "Computational Investigation of Blade Vortex Interaction Noise", AIAA Paper 99-0231, 38 ${ }^{\text {th }}$ Aerospace Sciences Meeting and Exhibit, Reno (NV), Jan. 10-13, 2000.

${ }^{28}$ Roshko, A., On the wake and drag of bluff bodies, J. Aeronaut. Sciences 22, 1955.

${ }^{29}$ Achenbach, E., Distribution of local pressure and skin friction around a circular cylinder in cross-flow up to $\operatorname{Re}=5 \times 10^{6}$, J. Fluid Mech. 34, 625-639, 1968.

${ }^{30}$ Zdravkovich, M. M., Flow around Circular Cylinders, Vol. 1 Fundamentals, Oxford University Press, 1997.

${ }^{31}$ Zdravkovich, M. M., Flow around Circular Cylinders, Vol. 2 Applications, Oxford University Press, 2003.

${ }^{32}$ Norberg, C., Interaction between Free-stream Turbulence and Vortex Shedding For a Single Tube In Cross-Flow, Journal of Wind Engineering and Industrial Aerodynamics 23, 1985.

${ }^{33}$ Norberg, C., Experimental investigation of the flow around a circular cylinder: influence of aspect ratio, Journal of Fluid Mechanics 258, pp. 287-316 96, 1994.

${ }^{34}$ Norberg, C., Flow around a circular cylinder: Aspects of fluctuating lift, Journal of Fluids and Structures $15(3-4)$, pp. 459-469, 2001.

${ }^{35}$ Norberg, C., Fluctuating lift on a circular cylinder: Review and new measurements, Journal of Fluids and Structures 17 (1), pp. 57-96 29, 2003.

${ }^{36}$ Breuer, M., Numerical and modeling influences on large-eddy simulations for the flow past a circular cylinder, Int. J. of Heat and Fluid Flow, 19:512-521, 1998.

${ }^{37}$ Kravchenko, A. G., Moin, P., Numerical studies of flow over a circular cylinder at $\mathrm{Re}=$ 3900, Physics of Fluids, 12:403-417, 2000.

${ }^{38}$ Franke, J., Frank, W., Large-eddy simulation of the flow past a circular cylinder at $\mathrm{Re}=3900$, Journal of Wind Engineering and Industrial Aerodynamics, 1191-1206, 2002.

${ }^{39}$ Beaudan P., Moin P., Numerical experiments on flow past a circular cylinder at-sub-critical Reynolds number, Technical report TF-62, Department of Mechanical Engineering, Stanford University,1994.

${ }^{40}$ Mittal, R., Moin, P., Suitability of upwind-based-finite difference schemes for large-eddy simulation of turbulent flows, AIAA Journal 35:1415, 1997.

${ }^{41}$ Kravchenko, A. G., Moin, P., B-spline methods and zonal grids for numerical simulations of turbulent flows, Report TF-73, Department of Mechanical Engineering, Stanford University, 1998. 
${ }^{42}$ Hoffman, J., Efficient Computation of Mean drag for The Sub-critical Flow Past a Circular Cylinder Using Adaptive DNS/LES, Int.J.of Num.Analys. and Modeling,1:1-18, 2004.

${ }^{43}$ Batcho, P., Karniadakis, G. E., Chaotic transport in two- and three-dimensional flow past a cylinder, Physics of Fluids A 3, 1051-1062, 1991.

${ }^{44}$ Mittal, R., Balachandar, S., Effect of three-dimensionality on the lift and drag of nominally two-dimensional cylinders, Physics of Fluids, 7:1841-1865, 1995.

${ }^{45}$ Williamson, C. H. K., 2-D and 3-D aspects of the wake of a cylinder, and their relation to wake computations. In C.R. Anderson and C. Greengard, editors, Lectures in Applied Mathematics, volume 28, pages 719-751, American Mathematical Society, 1991.

${ }^{46}$ Kalro, V., Tezduyar,T., Parallel 3D computation of unsteady flows around circular cylinders, Parallel Computing, 23,1235-1248, 1997.

${ }^{47}$ Prasad, A, Williamson CHK , The instability of the shear layer separating from a bluff body, Journal of Fluid Mechanics ,333:375-402, 1997.

${ }^{48}$ Jordan, S. A., Investigation of the cylinder separated shear-layer physics by large-eddy simulation, Int. J. of Heat and Fluid flow, 23:1-12, 2002.

${ }^{49}$ Singh, S., Mittal, S., Flow past a cylinder: shear layer instability and drag crisis, International Journal for Numerical Methods in Fluids 47:75-98, 2005.

${ }^{50}$ Catalano, P., Wang, M., Iccariono, G., Moin, P., Numerical simulation of the flow around a circular cylinder at high Reynolds numbers, Int. J. of Heat and Fluid flow, 24:463-469, 2003.

${ }^{51}$ Breuer, M., A Challenging Test Case for Large-eddy simulation: High Reynolds Number Circular Cylinder Flow, Int. J. of Heat and Fluid Flow, 21:648-654, 2001.

${ }^{52}$ Wang, M., Catalano, P., Iaccarino, G., Prediction of High Reynolds Number Flow over a Circular Cylinder Using LES with Wall Modeling, Center for Turbulence Research, Stanford Univ. Annual Research Briefs, 2001.

${ }^{53}$ Mittal, S., Kumar, V., Flow-induced vibrations of a light circular cylinder at Reynolds numbers $10^{3}$ to $10^{4}$, Journal of Sound and Vibrations, 245, 923-946, 2001.

${ }^{54}$ Lyrintzis, A., Xue, Y., Study of Noise Mechanisms of Transonic Blade-Vortex Interactions, AIAA Journal, Vo1.29, No.10, pp.1562-1572, 1990.

${ }^{55}$ Magagnato, E., Sorguven, E., Gabi, M., Far Field Prediction by Large-eddy simulation and Ffowcs -Willimas Hawkings Analogy, AIAA Paper 3206-2003, May 2003. 
${ }^{56}$ Lardeau, S., Leschziner, M.A., Unsteady Reynolds-Averaged Navier-Stokes Computations of Transitional Wake/Blade Interaction, AIAA, Vol.42, No.8, pp. 1559-1571, 2004, 2004.

${ }^{57}$ Smith, M. J., Sankar, L. N., "Evaluation of a Fourth-Order Compact Operator Scheme for Euler-Navier-Stokes Simulations of a Rotor in Hover, “AIAA Paper 91-0766, January 1991.

${ }^{58}$ Bennouna, M., White, R.G., The effect of large vibration amplitudes on the dynamic strain response of a clamped-clamped beam with consideration of fatigue life, Journal of Sound and Vibration 76, 281-308, 1984.

${ }^{59}$ Mei, C., Prasad, C.B., Effects of nonlinear damping on random response of beams to acoustic loading, Journal of Sound and Vibration 117, 173-186, 1987.

${ }^{60}$ Reinhall, P.G., Miles, R.N., Effects of damping and stiffness on the random vibration of non-linear periodic plates, Journal of Sound and Vibration 131, 33-42, 1989.

${ }^{61}$ Robinson, J., Mei, C., The influence of nonlinear damping on the random response of panels by time domain simulation, AIAA Paper 89-1104, 1989.

${ }^{62}$ Maestrello, L., Frendi, A., Nonlinear vibration and radiation from a panel with transition to chaos, AIAA Journal 30, 2632-2638, 1992.

${ }^{63}$ Frendi, A., Maestrello, L., Bayliss, A., Coupling between plate vibration and acoustic radiation, Journal of Sound and Vibration, 177, 207-226, 1994.

${ }^{64}$ Frendi, A., Coupling between a supersonic turbulent boundary layer and a flexible structure, AIAA Journal 35, 58-66, 1997.

${ }^{65}$ Frendi, A., Effect of pressure gradients on plate response and radiation in a supersonic turbulent boundary layer, NASA-CR-201691, 1997.

${ }^{66}$ Maestrello, L., Radiation from and panel response to a supersonic turbulent boundary layer, Journal of Sound and Vibration 10, 262-295, 1996.

${ }^{67}$ Frendi, A., Effect of wall shear stress on structural vibration. AIAA Journal 39, 737-740, 2001.

${ }^{68}$ Buhler, W., Frendi, A., Effect of fluid-wall shear stress on nonlinear beam vibration, Journal of Sound and Vibration 270, 793-811, 2004.

${ }^{69}$ Batina, J. T., Unsteady Euler Airfoil Solutions Using Unstructured Dynamic Meshes, AIAA Paper 89-0115, 27th AIAA Aerospace Sciences Meeting, January, 1989. 
${ }^{70}$ Ilie, M., Nitzsche, F., Matida, E., Aeroacoustic investigations of blade-vortex interaction using Large-eddy simulation, 14th AIAA/CEAS Aeroacoustics Conference (29th AIAA Aeroacoustics Conference), Vancouver, May 5-7, 2008.

${ }^{71}$ Ilie, M., Nitzsche, F., Matida, E., Aeroelastic response of an idealized airfoil to blade-vortex interaction; a CFD approach using Large-eddy simulation, accepted for the $49^{\text {th }}$ AIAA/ASME/ASCE/AHS/ASC Structures, Structural dynamics, and Materials Conference, Schaumburg, IL, April 7-10, 2008.

${ }^{72}$ Ilie, M., Nitzsche, F., Matida, E., Influence of angle of attack on the helicopter blade-vortex interaction mechanism of interaction using Large-eddy simulation, 46th AIAA Aerospace Sciences Meeting and Exhibit, Reno, Nevada, January 7-10, 2008.

${ }^{73}$ Ilie, M., Nitzsche, F., Matida, E., Two-Dimensional Blade-Vortex Interaction using Largeeddy simulation, $48^{\text {th }}$ AIAA/ASME/ASCE/AHS/ASC Structures, Structural dynamics, and Materials Conference, Hawaii, April 23-27, 2007. 